\title{
Valorization in public health genomics : a conceptual development from technology transfer to healthcare integration
}

Citation for published version (APA):

Lal, J. A. (2013). Valorization in public health genomics : a conceptual development from technology transfer to healthcare integration. [Doctoral Thesis, Maastricht University]. Maastricht University. https://doi.org/10.26481/dis.20131112jl

Document status and date:

Published: 01/01/2013

DOI:

10.26481/dis.20131112jl

Document Version:

Publisher's PDF, also known as Version of record

Please check the document version of this publication:

- A submitted manuscript is the version of the article upon submission and before peer-review. There can be important differences between the submitted version and the official published version of record.

People interested in the research are advised to contact the author for the final version of the publication, or visit the DOI to the publisher's website.

- The final author version and the galley proof are versions of the publication after peer review.

- The final published version features the final layout of the paper including the volume, issue and page numbers.

Link to publication

\footnotetext{
General rights rights.

- You may freely distribute the URL identifying the publication in the public portal. please follow below link for the End User Agreement:

www.umlib.nl/taverne-license

Take down policy

If you believe that this document breaches copyright please contact us at:

repository@maastrichtuniversity.nl

providing details and we will investigate your claim.
}

Copyright and moral rights for the publications made accessible in the public portal are retained by the authors and/or other copyright owners and it is a condition of accessing publications that users recognise and abide by the legal requirements associated with these

- Users may download and print one copy of any publication from the public portal for the purpose of private study or research.

- You may not further distribute the material or use it for any profit-making activity or commercial gain

If the publication is distributed under the terms of Article $25 \mathrm{fa}$ of the Dutch Copyright Act, indicated by the "Taverne" license above, 


\section{VALORIZATION \\ IN \\ PUBLIC HEALTH GENOMICS}

A Conceptual Development From

Technology Transfer

To

Healthcare Integration 


\section{(C) Copyright J.A. Lal, Maastricht 2013}

All rights reserved. No parts of this thesis may be reproduced, stored in a retrieval system, or transmitted in any form or by any means, electronic, mechanical photocopying, recording, or otherwise, without written permission from the author.

Material in this thesis with defined reference like 'published as' or 'adapted' belongs to the respective copyright owner as specified.

Front cover page template developed using Microsoft Powerpoint ${ }^{\circledast}$ 2010. Used with permission from Microsoft. Public Health/Medicine logo on front cover page taken from sweetclipart.com under the AttributionNonCommerical-ShareAlike 3.0 Unported License permission. DNA Microarray background on front cover page taken from Wikipedia.org (author Guillaume Paumier) under the Attribution-ShareAlike 3.0 Unported permission and the GNU Free Documentation License.

Last page cartoon developed using 'clip art' from Microsoft Powerpoint ${ }^{\circledR}$ 2010. Used with permission from Microsoft.

Back cover page bottom image taken from 'clip art' using Microsoft Powerpoint ${ }^{\circledR} 2010$ excluding triangle and texts. Used with permission from Microsoft. Back cover page top image of the bridge of Maastricht City taken from Maastricht University. Used with permission from Maastricht University.

The studies described in this thesis were supported by a grant from the European Commission PHGEN II (Public Health Genomics European Network) with duration period: June 2009 - November 2012 and EU-Project No. 20081302 as well as were supported by the Canadian Institutes for Health Research (CIHR), CIHR Institute of Genetics, the CIHR Institute of Health Services and Policy Research through a grant (no. ETG92250) to the APOGEENet/CanGèneTest Research and Knowledge Network on Genetic Services and Policy.

APOGEE-Net/CanGèneTest and the Institute for Public Health Genomics contributed towards the cost of publishing this thesis.

Printed by Smart Printing Solutions.

ISBN: 978-90-9027905-3 
"HE WHO OBSERVES THE WIND [AND WAITS FOR ALL CONDITIONS TO BE FAVORABLE] WILL NOT SOW. AND HE WHO REGARDS THE CLOUDS WIL NOT REAP"

Ecclesiastes 11:4 AMP 



\title{
Valorization in Public Health Genomics
}

\author{
A Conceptual Development \\ from Technology Transfer to Healthcare Integration
}

\author{
DISSERTATION
}

To obtain the degree of Doctor at

Maastricht University,

on the authority of the Rector Magnificus, Prof. Dr. L.L.G Soete,

in accordance with the decision on the Board of Deans,

to be defended in public

on Tuesday 12 November 2013 at 16:00 hours

by

Jonathan A. Lal 


\section{Supervisors}

Prof. Dr. Angela Brand MPH, Professor of Social Medicine \& Public Health Genomics, Founding Director of the Institute for Public Health Genomics, Department of Genetics and Cell Biology, Faculty of Health

Medicine and Life Sciences, Maastricht University

Dr. TMA Pai Endowed Chair in Public Heath Genomics and Adjunct Professor, Manipal Life Sciences Center, Manipal University, India

Prof. Dr. Servaas A. Morré, Professor of Host-Pathogen Genomics and Public Health, Director of the Institute for Public Health Genomics, Department of Genetics and Cell Biology, Faculty of Health Medicine and Life Sciences, Maastricht University Head of the Laboratory of Immunogenetics, Department of Medical Microbiology and Infection Control, VU University Medical Center, Amsterdam

\section{Assessment Committee}

Chairman

Prof. Dr. Joep P.M. Geraedts, Emeritus Professor of Genetics and Cell Biology, Former Head of the Cluster of Genetics and Cell Biology, Former Head of the Department of Clinical Genetics, Faculty of Health Medicine and Life Sciences, Maastricht University

Committee Members

Prof. Dr. Wiebe E. Bijker, Professor of Technology and Society, Department of Technology and Society Studies, Faculty of Arts and Social Sciences, Maastricht University

Dr. med Hans-Peter Dauben, Head German Agency for HTA (DAHTA) and Medical Innovation, German Institute for Medical Documentation and Information (DIMDI), Cologne, Germany

Dr. Harmen Jousma, Director Science-based Business, Faculty of Mathematics and Natural Sciences, Leiden University 


\section{Table of Contents}

$\begin{array}{lr}\text { Preface } & 9\end{array}$

$\begin{array}{ll}\text { List of Abbreviations } & 11\end{array}$

\begin{tabular}{l} 
1. Introduction \\
\hline
\end{tabular}

2. Chapters 39

PART A: ESTABLISHMENT OF THEORETICAL CONCEPTS 41

- $\quad$ Specific Aims

Chapter I: Public Health and Valorization of Genome-based Technologies: A New Model

45 Lal et al. Journal of Translational Medicine 2011, 9:207.

Chapter II: The Overarching Framework of Translation and Integration: a Case for the LAL

Model

Lal et al. Personalized Medicine 2013, In Press.

Chapter III: The LAL Model: from Hypothesis to Practical Steps for Implementation from basic Genome-based Evidences in Personalized Medicine Lal et al. Personalized Medicine 2013, 10(7):683-701.

Chapter IV: Conclusions

PART B: POTENTIAL IMPLEMENTATION OF LAL MODEL IN PRACTICE

- $\quad$ Specific Aims

Chapter V: Functional Dynamics: From Biological Complexity to Translation and Impact in Healthcare Systems Lal et al. Journal of Computer Science and Systems Biology 2013, 6(2):88-92.

Chapter VI: Chlamydia Trachomatis Infections and Subfertility: Opportunities to Translate Host Pathogen Genomic Data into Public Health Lal et al. Public Health Genomics 2013, 16:50-61.

Chapter VII: European Best Practice Guidelines for Quality Assurance, Provision and Use of Genome-based Information and Technologies - the 2012 Declaration of Rome Brand A, Lal JA. Drug Metabolism and Drug Interactions 2012, 27(3):177-182.

Chapter VIII: Conclusions Partly Adapted: Brand A, Lal J et al. The Newsletter of the British Society of Human Genetics 2013, (48):53-54.

3. General Discussion 
4. Concluding Remarks and Future Prospects

Adapted: Lal JA. The Newsletter of the British Society of Human Genetics 2013, (48):59-60.

5. Miscellaneous

i. Summary

259

ii. Dutch Summary

263

iii. Hindi Summary

267

iv. Curriculum Vitae

271

v. Publications

273

vi. Acknowledgements 


\section{Preface}

Personalized Healthcare has seen great growth in the past decade and is believed to continue so forth. Coming from working for Delft University of Technology as a Bioproduct Designer, we were trained towards bridging the gap between academics and industry and translating technologies in real time to industrial application as well as working with patents. We were quite focused on the commercialization of academic knowledge and patents and were contracted with industry and academic groups to commercialize or develop business plans of their in-house patents. To us the bridged gap between academic research and industry and the subsequent market penetration was the end goal. We did not think beyond those parameters as profit and success for the projects seemed inevitable.

Coming to Maastricht University, I was exposed to a totally new field of research, public health genomics and health policy research. For me, these were unheard terms and activities. With a background in Biotechnology, I was generally interested in genome-based technologies and initially wanted to integrate my past expertise at Delft with the current research. I noticed from now working in these new areas of research, healthcare implementation or diffusion was a totally different world in itself and as a Bioproduct Designer, such issues were not addressed.

On further investigation, I noticed that several of the bioproducts or genome-based technologies being commercialized for market penetration and generating good profits are in fact not implemented in healthcare or have delayed diffusion rates.

This gave birth to the title and work of this thesis. I hope that the reader will be intrigued by the present work and its importance regarding the issues the thesis addresses. Based on my now combined background, the preface can end on the following quote:

"No discipline seems pleasant at the time, but painful. Later on, however, it produces a harvest of righteousness and peace for those who have been trained by it"

Hebrews 12:11 NIV 



\section{List of Abbreviations}

ACCE

Analytical Validity, Clinical Validity, Clinical Utility, Ethical, Legal, Social Implications

APC

Antigen Presenting Cells

BDC

Basic Design Cycle

CAT

Chlamydia Antibody Test

CCL6

CC chemokine ligand 6

CCR1

Chemokine Receptor 1

CCR5

C-C Chemokine Receptor Type 5

CDC

Centers for Disease Control and Prevention

CE

Conformtie Europeene or European Conformity Marking

$\mathrm{Cl}$

Customer Intelligence

CLV

Customer Lifetime Value

CpG

Cytosine Phosphate Guanine

CT

Chlamydia trachomatis

CTA

Constructive Technology Assessment

CVM

Customer Value Management

DAMP

Danger Associated Molecular Pattern

DG SANCO

Directorate-General for Health and Consumers

DM

Decision Makers

EAHC

European Agency for Health Consumers

EEA

European Economic Area

EFTA

European Free Trade Association/Area

EGAPP

Evaluation of Genomics Applications in Practice and Prevention;

EGC

EpiGenChlamydia

ELSI

Ethical, Legal, Social Implications

EMA

European Medicines Agency

EPC

Enhanced Primary Care

ESPT

European Society for Pharmacogenomics and Theranostics

EU

European Union

FDA

Federal Drug Administration 


\begin{tabular}{|c|c|}
\hline FP & Framework Program \\
\hline FTA & Free Trade Area \\
\hline FTM & Fish Trap Model \\
\hline GBI & Genome-based Information \\
\hline GBITs & Genome-based Information and Technologies \\
\hline GBTs & Genome-based Technologies \\
\hline HIA & Health Impact Assessment \\
\hline HiAP & Health in All Policies \\
\hline HLA & Human Leukocyte Antigen \\
\hline HNA & Health Needs Assessment \\
\hline HS & Horizon Scanning \\
\hline HSG & Hysterosalpingography \\
\hline HTA & Health Technology Assessment \\
\hline ICT & Information and Communication Technology \\
\hline ICTI & Integrated Approach to the study of Chlamydia Trachomatis Infection \\
\hline IFN & Interferon \\
\hline $\lg G$ & Immunoglobulin G \\
\hline IL & Interleukin \\
\hline IPR & Intellectual Property Rights \\
\hline ITFoM & Information and Communication Technology Future of Medicine \\
\hline IVD & In-vitro Diagnostics \\
\hline IVF & In-vitro fertilization \\
\hline$J A$ & Joint Action \\
\hline $\mathrm{LAL}$ & Learning-Adapting-Leveling \\
\hline LPS & Lipopolysaccharide \\
\hline MBL & Mannose-Binding Lectin \\
\hline MICA & Major Histocompatibility Complex Class I Chain-related A \\
\hline NTF & National Task Force \\
\hline P4 & Predictive, Personalized, Preemptive and Participatory \\
\hline PAMP & Pathogen Associated Molecular Pattern \\
\hline PGN & Peptido-Glycan \\
\hline
\end{tabular}




$\begin{array}{ll}\text { PHAT } & \text { Public Health Assessment Tools } \\ \text { PHG } & \text { Public Health Genomics } \\ \text { PHGEN II } & \text { Public Health Genomics European Network } \\ \text { PHGW } & \text { Public Health Genomics Wheel } \\ \text { PPP } & \text { Public Private Partnership } \\ \text { PRR } & \text { Pattern Recognition Receptor } \\ \text { QoE } & \text { Quality of Evidence } \\ \text { R\&D } & \text { Research and Development } \\ \text { RANTES } & \text { Regulated on activation, normal T expressed and secreted } \\ \text { ROI } & \text { Return on Investment } \\ \text { SMEs } & \text { Small Medium Enterprises } \\ \text { SNP } & \text { Single Nucleotide Polymorphism } \\ \text { SWOT } & \text { Strengths Weakness Opportunities Threats } \\ \text { T1-T4 } & \text { Translation research 1 to Translation research } 4 \\ \text { TLR } & \text { Toll-like Receptors } \\ \text { TNF- } \alpha & \text { Tumor Necrosis Factor alpha } \\ \text { TP } & \text { Tubal Pathology } \\ \text { TT } & \text { Technology Transfer } \\ \text { Vol } & \text { Value of Information } \\ \text { WHO } & \text { World Health Organization } \\ \end{array}$





\section{Section 1}

\section{Introduction}

Background

General Aims

Overview of Thesis Chapters

References 


\section{Outline}

Within public health, and especially in Public Health Genomics, personalized health interventions have become of great importance recently and will be in the future. However, their applications and implementation in healthcare systems seem limited and/or delayed. Consequently both industry and individuals are at disadvantage here as a result. This issue defines the importance of this work. The thesis aims to address the issue of this bottleneck by developing a conceptual framework and demonstrating its potential implementation for public health. In the process it aims to resolve the bottleneck of personalized health application. This will be further emphasized in the subsequent paragraphs. This chapter will start off with some background on Public Health Genomics and on how personalized healthcare was derived through the complexity of biology, which gave rise to the 'omics' sciences and eventually systems biology leading to the demand of personalized medicine and beyond. The chapter then will define the state of the art of the various tools and activities used in translational research for moving personalized healthcare from bench to healthcare. This will be followed by elaborating on the problem statement, i.e. the backlog of personalized health applications, and will then define the objectives of the thesis.

\section{Omics, Systems Biology to Personalized Healthcare}

Molecular biology in combination with other basic sciences as supplements, both human and nonhuman research has greatly contributed not only to medical and applied research as well as directly to medicine and drug discovery, but also to healthcare as a whole. Historically this has been well documented from the early times for example the advent of genetics to disease, molecular coding, viruses and the molecular mechanism of infection, protein structure, etc. [1] to examples in modern application, like diabetic complications. For example, glucose mediated vascular damage has been attributed to four pivotal molecular mechanism providing a potential for drug discovery [2]. However, only in the past few decades, due to the birth of the concept of genomics and other 'omics' ${ }^{1}$ we have come to know beyond what was possible with just basic molecular biology largely due to the potential of computational power $[3,4]$. Similarly drug discovery applications historically have been influenced significantly through genomics [5]. This led to the hype of the future of genomics research [6, 7]. As a

\footnotetext{
${ }^{1}$ Omics encompass all types like Genomics, Proteomics, Metabolomics, Transcriptomics, etc.
} 
consequence of this expansion, we have come to know about the complexity of biology bringing in more questions than answers, in this huge network of molecular interactions resulting in functional consequences $[8,9]$.

These collective 'omics' including their interactions with environmental factors (exposome, epigenomics, microbiome, lifestyle, etc.) and the complex knowledge generated demanded for a more holistic systems approach and large scale models to evaluate information and to understand function [10] propelling the concept of systems biology. Systems biology is defined as an integrative, interdisciplinary approach to biological science that is built around the concept of close integration of computational methods, technology development (including -omics) and global measurement and analysis of biological systems [11].

The drive of systems biology and 'omics' power has inclined medical research towards a more personalized approach to healthcare $[11,12]$. With the advancement of 'omics' and access to information via the internet and social media, the individual is demanding a more personalized approach to his/her treatment [13] given we now know that every person is unique. Such advancement has led to the development of the field of personalized medicine and healthcare, which is defined as the use of genome-based ${ }^{2}$ information and technologies ${ }^{3}$ for providing more stratified and precise (and possibly truly personalized) interventions [14]. Through the systems approach to medicine, medical application is moving towards a more predictive, personalized, preventive and participatory (P4) approach [15]. As a result, in 2009 alone, the U.S personalized medicine market was at $\$ 232$ billion and is projected to reach $\$ 452$ billion by 2015 [16]. Consequently, in this post-genomic era through systems biology and 'omics', personalized healthcare which also includes personalized medicine is becoming the way to move forward in prevention and treatment of disease in a healthcare setting.

\section{State of the Art for Translational Research}

To realize P4 medicine or personalized healthcare, one must move this knowledge from the laboratory into healthcare systems for daily practice. Translational research is defined as the effective translation of

\footnotetext{
${ }^{2}$ Genome-based is in a synonymous with Omics as everything deriving from the human genome is genome-based, i.e. transcriptomics, metabolomics, etc.

${ }^{3}$ Information also includes omics interactions with the environment and technologies can include a diagnostic application, intervention, technique, process, methodology, clinical application, and technology in the general sense
} 


\section{| INTRODUCTION}

new knowledge, mechanisms and techniques generated by advances in basic science research into new approaches for prevention, diagnosis and treatment of disease essential for improving health [17]. Translational research is considered pivotal for the realization of shift from bench to bedside and beyond, i.e. to healthcare, of personalized health interventions. There are several methodologies or the state of the art in translational research at different stages of the transition from bench to healthcare, which will be described in the subsequent paragraphs. This is divided into two sections, namely valorization focusing on the commercialization to the market and then the healthcare implementation tools focusing on the integration aspects into healthcare.

\section{Valorization}

In translational research, valorization is a term commonly found responsible for bringing research ideas into science-based businesses onto the market (bench to market). Valorization can be defined as the transformation of knowledge into concrete new products, services and processes [18]. The term is generally associated with commercialization of knowledge/technology from an academic or industrial environment. Through systems biology and 'omics' research, we see the knowledge generated is being valorized into personalized health applications on the market through the biotechnology sector [19-22]. Valorization as such does not have a defined methodology rather a concept put into action using other methods. Terms or methodologies related or overlapping with valorization are regional valorization systems, open innovations, technology transfer, spin-offs and drug discovery, all of which will be emphasized on in the following paragraphs.

\section{Regional Valorization Systems}

To achieve knowledge valorization, there is no defined methodology. However, one related conceptual framework for knowledge valorization is called a Regional Valorization System. The idea is that interactions between different regional actors will streamline knowledge transfer from academics to business and subsequent policy development for such knowledge valorization to business. A Regional Valorization System [23] is a framework, which aims to achieve knowledge valorization between academia and the business sector by providing support at different sub-systems as well as to make aware important areas and interactions. Furthermore, it aims to involve local and regional governing bodies to recognize the urgency on the direction of knowledge based development and subsequent policies. Smooth interaction between the different sub-systems leading to knowledge valorization from academics to business can be seen as the success of this framework. The actors required and available to 
facilitate this framework include interactions of people from multiple disciplines for example, basic and applied university researchers, industrial partners, hospitals, contract researchers, venture capitalists, funding agencies including both non and governmental, human resource department, policy makers and local government. The expectation of the stakeholders within this framework is consistent and clear communication and interactions between the different players involved to facilitate knowledge valorization.

\section{Open Innovations}

Another term related to valorization is open innovation. Open innovation [24] can be defined as to profit from external knowledge without making heavy internal investment in long term research. This may include any form of cooperation with third parties that can contribute to improve the long term performance of a company as well as grants both governmental and NGOs. Cooperation with third parties, for example contracted research to private institutes, companies or universities will help in avoiding substantial investment in one's own infrastructure to do research to develop say a product. Licensing production is another example. Stakeholders expect minimal amount of investment with maximal amount of returns. Consistency can vary from case to case as well as organization to organization. Current trends (patents, trademarks and trade secrets among others) limit the full potential for open innovation.

\section{$\underline{\text { Technology Transfer }}$}

Valorization also closely associates with another term called Technology Transfer (TT). Technology Transfer aims to transfer technology from one organization to another organization. TT is seen as an activity of the migration of early discoveries in any setting (e.g. private sector, academia) to useful application in the development of marketable products or processes (adapted from [25]). Again this is on the business side of translational research and overlaps with the concept of valorization but generally has defined methods. Various $\Pi$ methodologies are well known and actively utilized by the commercial sector to move ideas from the lab onto the market. For an example see figure 1 [26]. Furthermore, TT offices exclusively also exist both in academics as well as private sector just to help in commercializing an idea or patent or technology. Other resources include investors as well as development of business models. Staff activities can involve coming up with a concept which addresses a market need and subsequently developing that concept. The concept of $\Pi$ is well established and proven [27]. Stakeholders expect that the product developed through the TT pipeline is eventually rolled into the 
market and successfully commercialized for return on investment. Consistency of this performance is based on a case by case basis and the market need. For example, in the biopharmaceutical industry, it does not have such a high success rate, whereas in the medical devices and electronics markets it has more consistency in the same order. It is to be noted that TT is differentially applied and may not have the same order as figure 1 below.

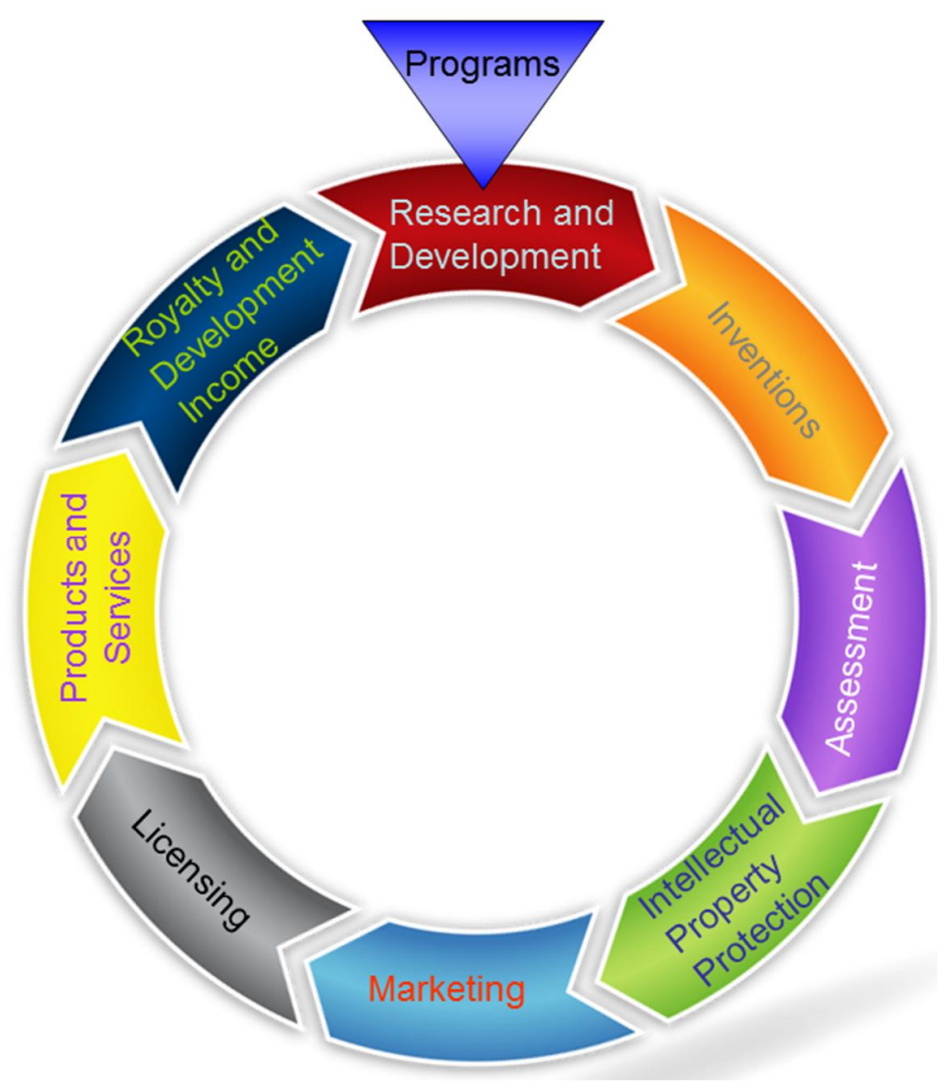

Figure 1: A sketch of an example of technology transfer taken from [26]. The idea is that research in the lab leads to an idea, which is developed into an invention and assessed for its feasibility. After or during the development of the technology, the technology is protected by patenting, thereby marketing the technology to customers. These customers can take the license of the technology to sell it to other customers or can be skipped. This results in products and services eventually leading to return on investments.

$\underline{\text { Spin-offs }}$

Spin-off is a term, which partly overlaps with the concept of valorization in the specific setting of academia, although does not have an absolute defined methodology. Spin-offs have been extensively researched can be considered an emerging field of work focusing on the process of creating, discovering and exploiting technological and scientific opportunities created by academic research through the development of an independent company created from an existing part or person of the academic institution or company. Spin-offs can be considered almost synonymous with SMEs (Small Medium Enterprises) as SMEs generally are the companies, which are created as a result of the endeavor. Therefore, spin-offs can be defined as firms whose products or services develop out of technology-based 
ideas or scientific/technical know-how generated in a research setting by a member of the research institution, staff or student who founded (or co-founded with others) the firm [28]. The term firm can be replaced with the term SMEs. Spin-offs are related to technology transfer or even overlapping greatly with technology transfer in some instances based on the latter's methodology. For spin-offs in some cases, academic resources may be required or not for conceiving the initial research idea based on the market need or creating the need. Investments like from venture capitalists, grants, etc. in the development of a business model for a company based on the concept of the product are generally required. Technology transfer offices (TTOs) are available for such a venture and spin-offs generally require approval from the research institution.

\section{Drug Discovery}

Knowledge valorization also relates to the development and discovery of biopharmaceuticals. Moving a drug from the lab to the market is generally done in phases, which seem to overlap again with the above mentioned concepts. Drug discovery phases can be seen from figure 2 [29] below. Like $T$, drug discovery has a defined yet subjective methodology. After preclinical research, a medical target is identified which can be exploited for drug development purposes. Thereafter, the feasibility of the target for drug development is assessed leading to preclinical development and eventual creation of the drug. Phase I concerns the test of the drug for safety, administered amount and side-effects in a volunteered small healthy group of subjects. Phase II is concerned with effectiveness as well as safety in a volunteered small healthy group of subjects. Phase III involves testing the drug on a large group of volunteered subjects. This is to establish the effectiveness as well as to observe side-effects and compare to currently used treatments. Phase IV is done when the drug is approved and use has started. The concept is to gather and analyze data on risks, benefits and best usage.

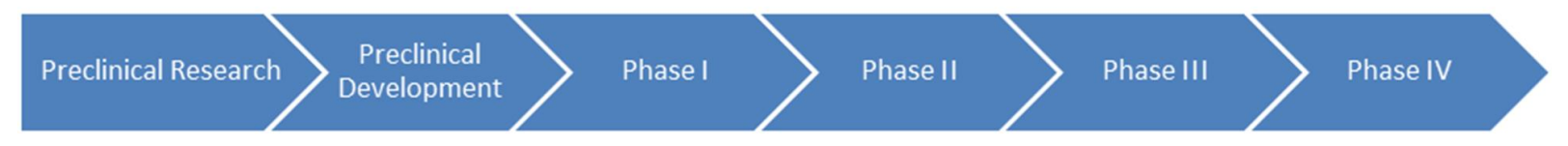

Figure 2: The different stages of drug discovery and development. Taken from [29]

\section{Healthcare Implementation Tools}

The above mentioned terms generally define the most generally used mechanisms in the commercialization of knowledge and technologies. However, only technology transfer and drug discovery have a defined methodology, which overlaps in concepts between the remainder of terms as 


\section{| INTRODUCTION}

can be seen. So there seems to be a possibility of interchangeable terms in the process of commercialization. On the other hand of translational research, implementation or integration of technologies into healthcare beyond commercialization has several activities such as Health Needs Assessment (HNA), Health Technology Assessment (HTA), Health Impact Assessment (HIA), ACCE, EGAPP, T1-T4, Public Health Genomics Enterprise, and the Public Health Wheel. These will be elaborated upon in the subsequent paragraphs.

\section{Health Needs Assessment}

Major tools used by decision makers directly or indirectly include Health Needs Assessment (HNA), Health Technology Assessment (HTA) and Health Impact Assessment (HIA) [30]. HNA is 'a systematic method of reviewing the health issues facing a population, leading to agreed priorities and resource allocation based on the needs of the population that will improve health and reduce inequalities' [31]. HNA can use epidemiological, qualitative and comparative methods to this end to address the above definition [32]. HNA works towards influencing policy through logical decision making, promoting interagency collaboration and advance prioritized R\&D [33]. The methodology of HNA involves first estimating the frequency of health issues in a given population and then evaluating the proof for the positive and negative effects of the technologies per health issue [34]. In the process, HNA addresses the impact, changeability, acceptability and resource feasibility with respect to maximum positive impact the technology can have on the health of a population, i.e. targeting whole populations, subpopulations as well as individuals [31].

\section{Health Technology Assessment}

Health Technology Assessment (HTA) is considered a very important tool looked at by decision makers. HTA can be defined as a 'multidisciplinary process that summarizes information about the medical, social, economic, legal and ethical issues related to the use of a health technology in a systematic, transparent, unbiased, robust manner' [35]. HTA strives for the best possible value and also works on informing in the development of effective and safe (health) policies that are focused on the patient/citizen [35]. As this applies HTA's involvement in interventions and appraisals it is considered as an important information tool for decision-making [30]. According to Battista [36] HTA could be perceived to bridge the gap between decision-making and research, however, it is perceived to be based in scientific method [35]. Policy analysis, (health) economic evaluation and evidence-based medicine play a pivotal role in HTA. This is also extended to humanities/social sciences as well [37]. HTA is 
complimented by horizon scanning (HS - identifying emerging and new technologies) [38] and Constructive Technology Assessment (CTA). The latter addresses the issue of technologies being highly dynamic [39]. Historically HTA was developed from the need of healthcare systems and/or decision makers to identify relevant technologies for their usage [40, 41]. An example of the HTA process can be seen from figure 3 below [42].

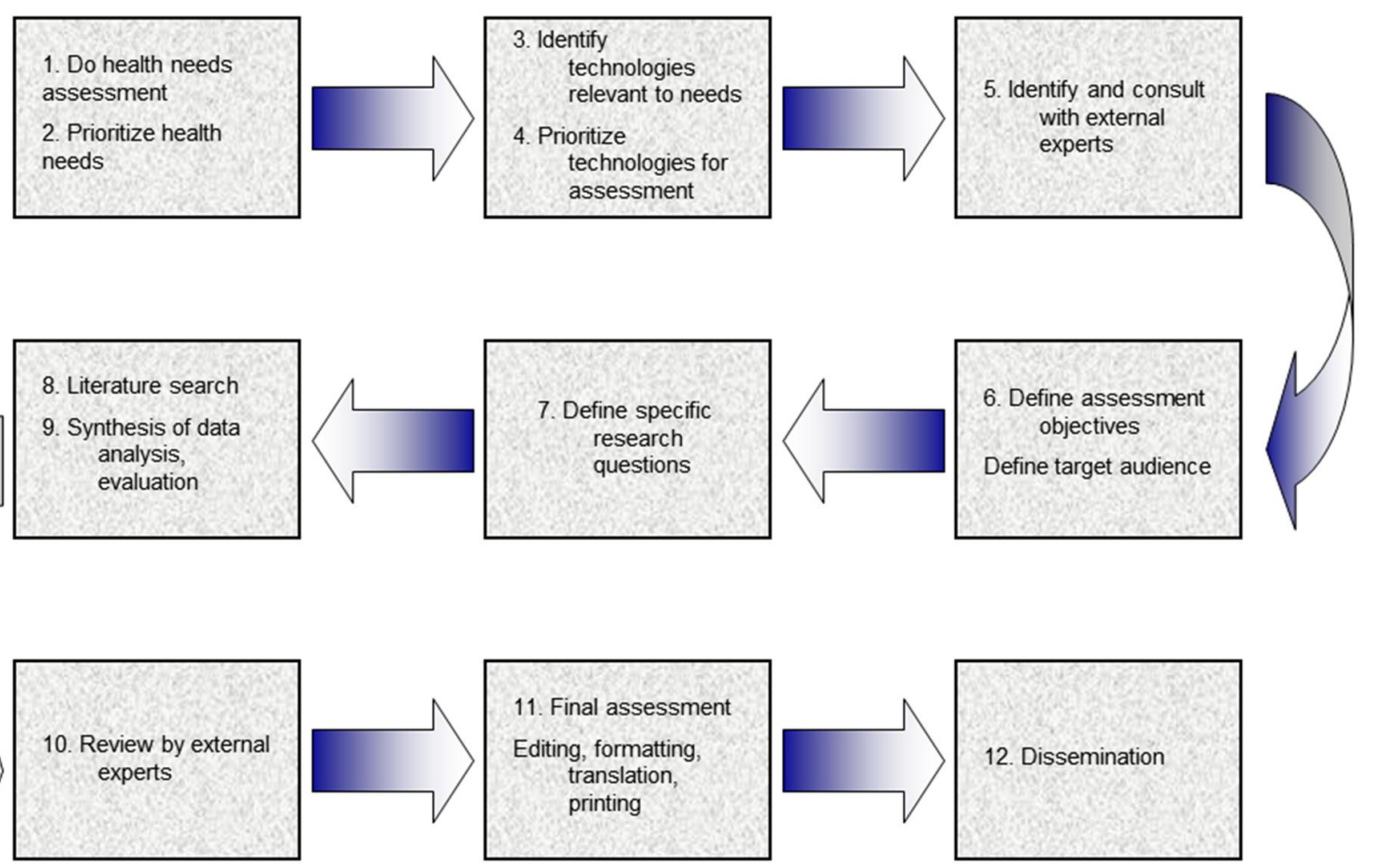

Figure 3: A simplified example of an HTA process. Taken from [42]. Different agencies can do HTA differently. It is to be noted that the HNA part of HTA is different from the general HNA.

\section{Health Impact Assessment}

Like HNA and HTA, Health Impact Assessment (HIA) is a good evaluation tool used by decision makers. HIA is 'a combination of procedures, methods and tools by which a policy, program, or project may be judged as to its potential effects on the health of a population, and the distribution of those effects within the population' [43]. In other words, HIA gives decision makers insights of the full spectrum of consequences of genome-based technologies (GBTs) or policies as a result, as well as informs about unpredictability [44]. HIA, as a tool first investigates and determines various polices which can positively or negatively impact health. This is followed by considering both, direct as well as indirect health effects, thereby determining, which populations are affected as a result and on what basis. This is followed by 
reporting the findings to decision makers and finally evaluating the result of actual implementation [32]. HIA originated partly from environmental policy development, health equity and a social view of health [45]. In conclusion, HNA identifies 'health priorities for a given population', HTA evaluates the 'performance of health care technologies' and HIA assesses the 'effects of policies, programs or projects on the population's health' [32]. There are professionals and organizations, some designated by policy makers working on these separate 3 yet overlapping tools to develop recommendations for decision makers.

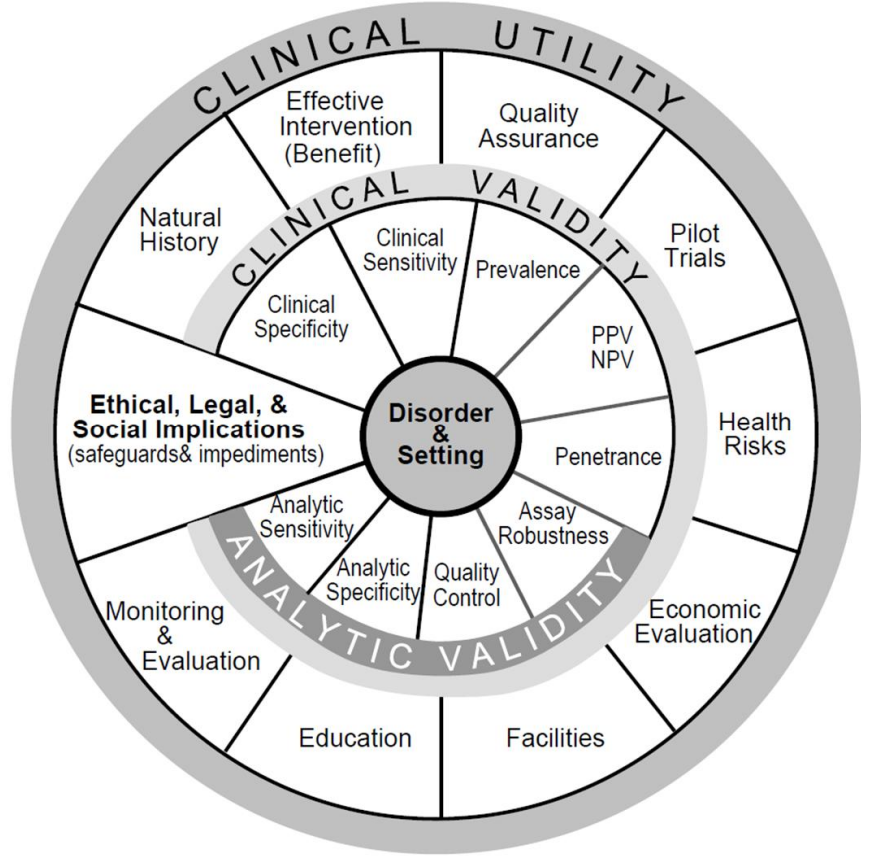

Figure 4: Sections of the ACCE model and their details. The figure shows what falls under analytical validity, clinical validity, clinical utility and ELSI. Taken from [47]

On the other hand, the ACCE (Analytical Validity, Clinical Validity, Clinical Utility, Ethical, Legal, and Social Implications, see figure 4) is considered a benchmark framework to evaluate specifically emerging genetic tests [46]. Supported and developed by CDC, USA, it aims to inform decision makers by presenting data on genetic tests in an updated readable format for them to make decisions [47]. From ACCE, Analytical Validity defines 'how accurately and reliably the genetic test measures the genotype of interest' [47]. Clinical Validity defines 'how consistently and accurately the test detects or predicts the intermediate or final outcomes of interest' [47]. On the other hand, Clinical Utility defines 'how likely the test is to significantly improve patient outcomes' [47]. The last ' $E$ ' in the ACCE stands for ELSI (Ethical, 
Legal, and Social Implications) and forms an important part of the ACCE framework as implications may arise in context of using the genetic test [47]. The ACCE methodology includes gathering data, assessing it and interpreting the results followed by reporting that data to decision makers in an understandable updated read for them to come to a decision. This data is generally about DNA-related testing or genetic disorders [47]. A standard set of 44 specific questions comprises the model. These 44 questions are divided into 5 sections namely, disorder/setting (7 questions), analytical validity (10 questions), clinical validity (8 questions), clinical utility (16 questions) and ELSI (3 questions). Some of these 5 sections are also further divided into subsections [47].

\section{EGAPP}

EGAPP (Evaluation of Genomics Applications in Practice and Prevention) established in 2004 [48], can be in a way considered as the successor to the ACCE model as it builds upon ACCE and was also developed by CDC, USA. The EGAPP working group aims at the development of a 'systematic, evidence-based process for assessing genetics tests and other applications of genomic technology in transition from research to clinical and public health practice' [48]. The methodology constitutes existing recommendations based on population-based epidemiological studies as well as knowledge from prior initiatives [48]. EGAPP consists of independent multidisciplinary panel of 15 experts sanctioned through CDC. The general methodology involves selecting potential topics for review through horizon scanning and feedback, and then defining disorders of interest, specific tests and clinical scenario. This is followed by brief summaries of identified tests and a preliminary review and prioritization of topics using specific criteria ending in voting. The next step involves the EGAPP evidence review process. This starts with formulation of key questions and analytical framework for evidence review followed by a systematic evidence review either in the direction of comprehensive EPC (Enhanced Primary Care) reports or Rapid/Targeted reviews. After this, the EGAPP reviews the evidence reports, considers contextual issues and may consider other sources of evidence, and as a result a draft recommendation peer-reviewed statement is developed and published. This ends with the dissemination to stakeholders. Detailed step by step can be found in the document [49] CDC published.

\section{$\underline{\mathrm{T} 1-\mathrm{T} 4}$}

Khoury et al. [50] propose a framework for the continuum of multidisciplinary translation research based on population-based epidemiological studies. Although not part of the assessment used by decision makers, this framework has received attention [32]. The authors divide their framework into 4 phases. 
Phase 1 translation research (T1) aims to move a 'basic genomic discovery into a candidate health application'. This is followed by phase 2 (T2), which 'assesses the value of a genomic application for health practice leading to the development of evidence-based guidelines'. Phase 3 (T3) moves from 'evidence-based guidelines to health practice'. The final phase 4 (T4) evaluates in practice the health outcomes the genomic application. T1 to T4 can have feedback loops [50]. Khoury et al. compares their phases with drug discovery and ACCE mentioned above as we as the Human Genome Project (HuGE), which can be seen from figure 5 below.

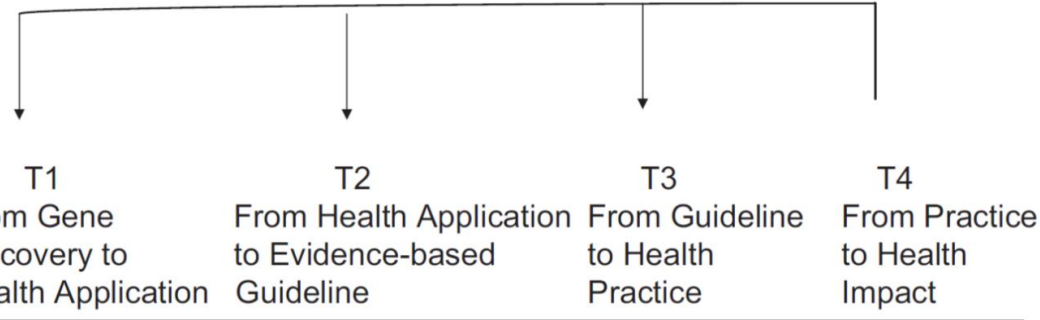

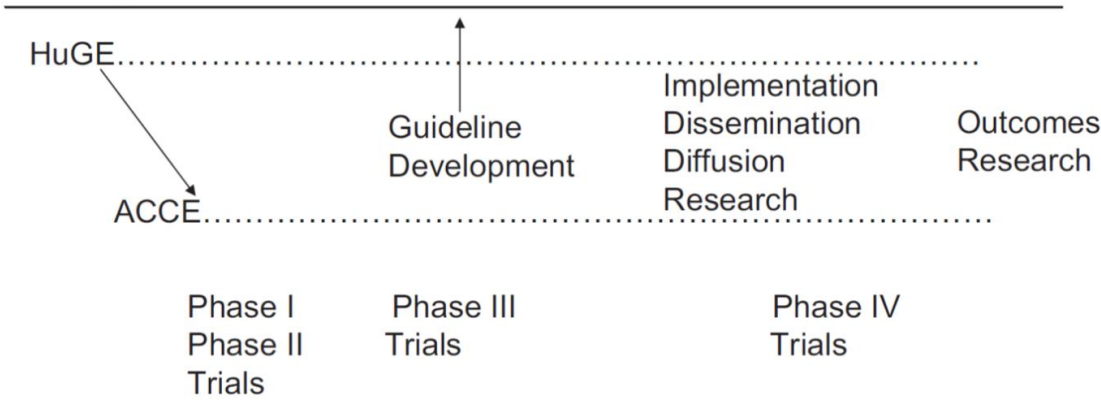

Figure 5: Giving an example of T1 to T4 over different tools like ACCE and drug discovery. Taken from [50]. It should be noted here however, T1 to T4 does not have a defined methodology.

\section{The Public Health Genomics Enterprise}

The Public Health Genomics (PHG) Enterprise (see figure 6 below) is a composite 'for effective translation of genome-based knowledge and technologies into improved population health' [51]. The consensus was developed by an international expert workshop held in Bellagio, Italy in 2005, with 18 experts from US, Canada, Germany, UK and France. Although not part of the assessment process by policy makers, it nonetheless contains important components and overlaps with tools previously mentioned. The PHG Enterprise's knowledge integration is considered pivotal [51]. It can be defined as the process of selecting, storing, collating, analyzing, integrating and disseminating genome-based information both within and across disciplines for the benefit of population health'. This can also constitute methodological progression as well [51]. Further information can be seen from figure 6 below. 


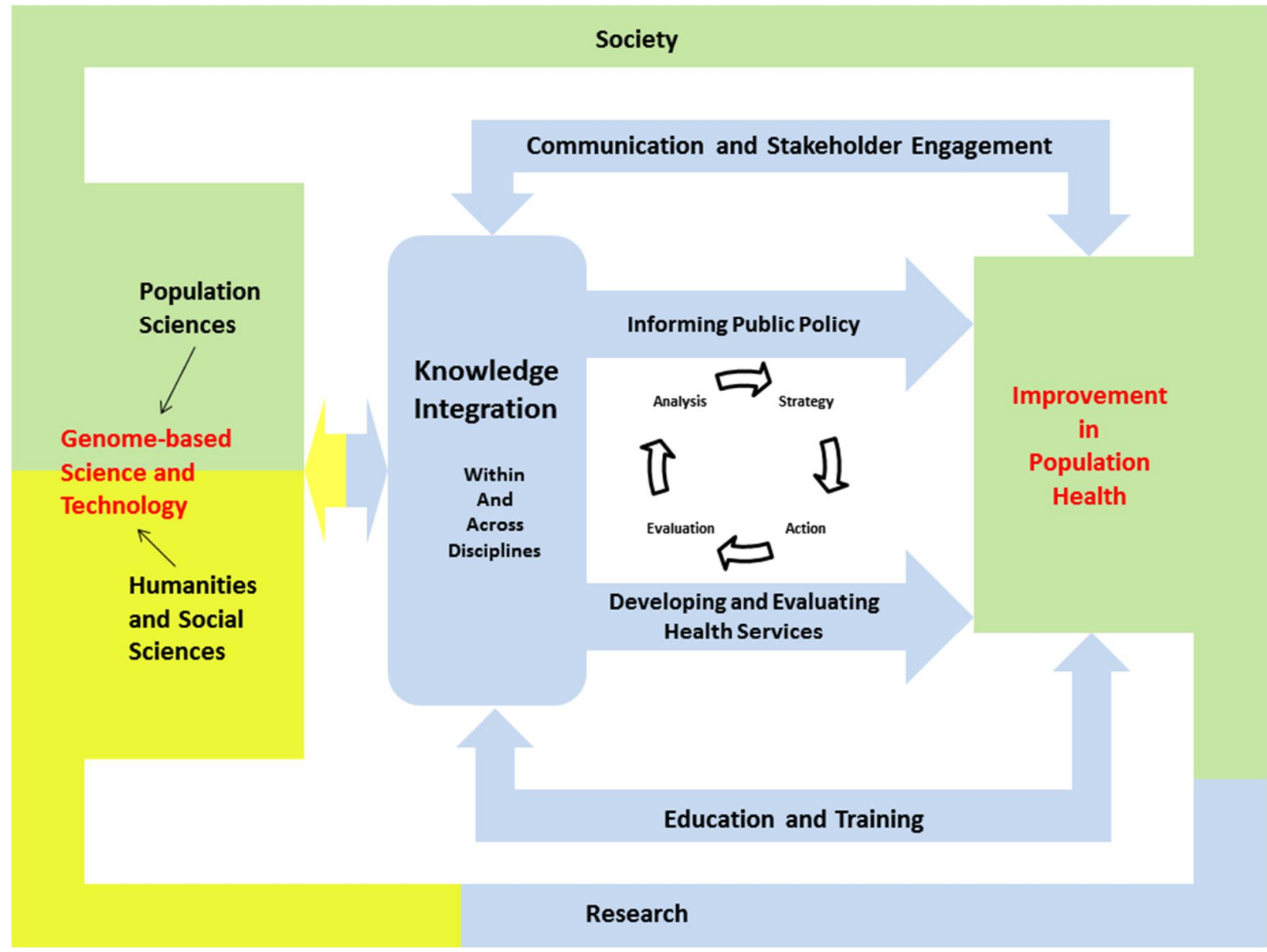

Figure 6: The PHG Enterprise - Blue represents the scope of the enterprise, yellow indicates creation of knowledge through research and red indicates genome-based science and technology playing a pivotal role. Green indicates society in its widest sense including stakeholders, activities, etc. Two sided arrows indicate dynamic and interactive nature of the enterprise. As can be seen the PHG enterprise has four main activities, viz. communication and stakeholder engagement, informing public policy, developing and evaluating preventive and clinical health services as well as education and training. Research identifies gaps with differentiation between basic and applied research being blurred. Also the generated knowledge is modulated by the results of its own output. This includes a cycle of analysis, strategy, action, evaluation, which is widely regarded in public health practice. Taken from [51].

\section{The Public Health Wheel}

The Public Health Wheel [52] can be seen from figure 7. The PHG Wheel demonstrates the integration of genome-based information and technologies into public health by addressing the 10 essential tasks of public health in the translation of the technology in question. 


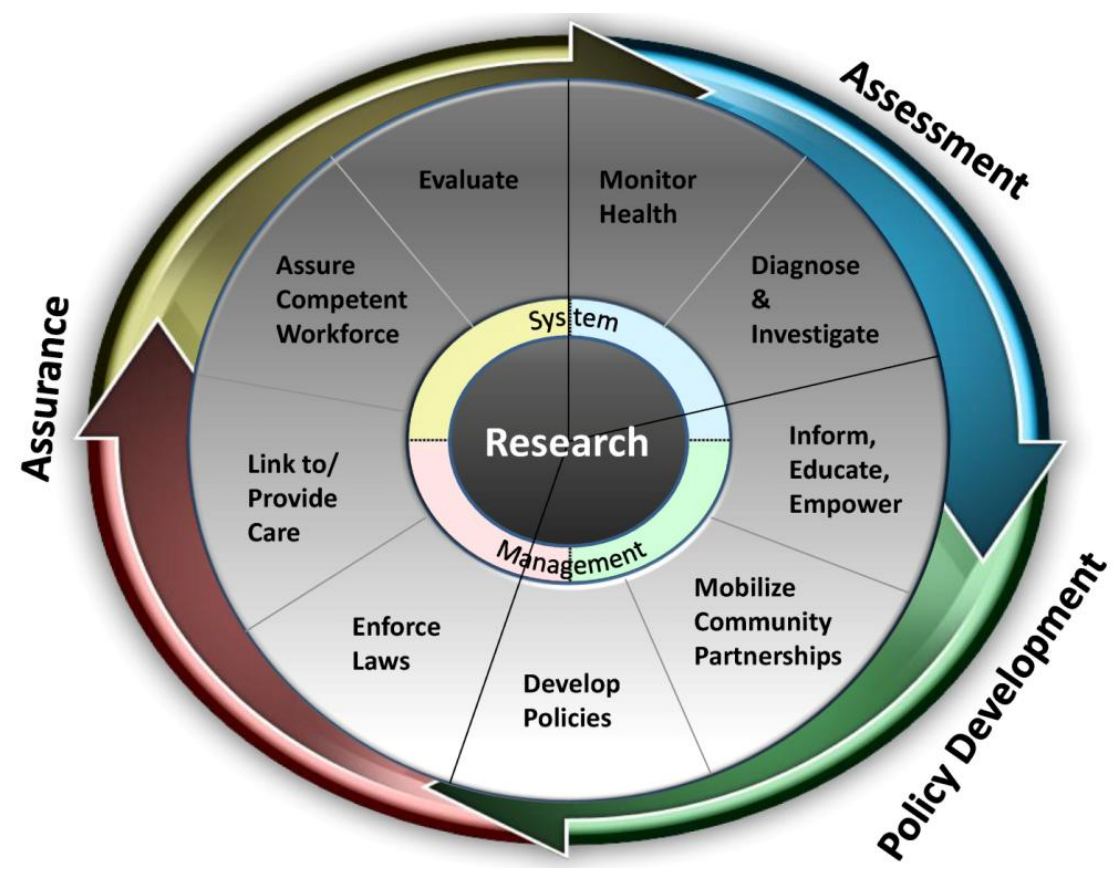

Figure 7: The Public Health Wheel which divides 10 essential tasks of public health over the domains of assessment, policy development and assurance with research at its core. The idea is that by addressing these 10 essential tasks, integration of genomics into public health can be possible. Taken from [52].

\section{The Problem Statement}

The above mentioned tools summarize the most popular activities or tools for translational research of health interventions from bench (lab) through the market to bedside and beyond (healthcare). As earlier stated in the outline of this chapter and the subsequent paragraphs, it is seen that the importance of personalized healthcare and its exponential market is being realized. Furthermore, there are various tools, some of which overlap that can bring personalized health applications from bench to healthcare. Still however, the timely implementation of these applications depending upon the type of technology seems apparently delayed. This is evident from large amount of scientific data in the market $[21,22]$ and also in terms of literature [53], patents [54,55] and publications compared to the uptake by hospitals and the healthcare system [56]. There seems to be a backlog of technological integration into the healthcare system. As a result, by the time a new genome-based technology arrives in practice, it becomes outdated. Even though the technology may get part/whole of the job done, there is always a newer technology waiting to be used, which may get the same/better results more efficiently in terms of time, accuracy, specificity and costs (notwithstanding the cost of the technology itself) and this makes the difference in the quality of life. Even guidelines developed tend to focus on handling and use genome-based information no matter by what type of technology the information was produced. 
Public Health Genomics is the responsible and effective translation of genome-based knowledge and technologies into public policy and health services for the benefit of population health [51]. It is an emerging field with increased demands of integration of genome-based information into public health due to the prior's increasing value and relevance in the latter. The field of Public Health Genomics based on its definition exactly answers the problem. This issue of genome-based technologies, (which include personalized health interventions), delayed translation and implementation in healthcare is an important problem to be addressed. Both healthcare and industry is as a loss here, if relevant technologies are not transferred in real time to the healthcare settings. For example, patients can benefit from the latest personalized medicine applications if provided on time, possible focusing on early prevention rather than only cure, thus possibly alleviating the burden of disease. Also industry can generate larger profits if timely interventions reach the patient or citizen as compared to delayed sales or limited sales. It becomes indeed a pity if the boom of personalized healthcare including personalized medicine with its huge market and promises is delayed. Therefore the aim of this thesis is to address this backlog through the concept and still relatively new field of public health genomics.

\section{The Missing Link}

The thesis approaches the issue with the concept of bringing valorization in public health genomics to complete the picture. Reason being valorization addresses the business side of things including commercialization, whereas the definition of public health genomics concentrates on the translation into public policy and health services. Also cross-integration of different concepts has been suggested [57] to bring in the effective and efficient real-time integration of genome-based technologies. Based on the problem statement, there seems to be a rabbit and turtle pace between the two enterprises, namely, the science-based industry and healthcare system respectively when it especially comes to personalized healthcare applications. The thesis will address the problem of the backlog and bottleneck of the real time effective and efficient integration of genome-based information and technologies (including personalized medicine and healthcare applications) from the bench (lab) through the market to the healthcare covering the whole spectrum of translational research of health interventions. 
The thesis aim to address the issue of the backlog and bottleneck of the effective and efficient integration of genome-based information and technologies from the first idea, through the market, to implementation in healthcare covering the whole spectrum of translational research. The hypothesis is that given the exponential increase in genome-based technological innovation, systems in place for technological integration into healthcare practice still have a backlog in real time. The thesis therefore projects to gain knowledge about current methodological apparatuses in place, identification of gaps/problems and issues in healthcare systems, see possible integration of relevant tools and give recommendations. Furthermore, certain existing concepts and ideas have overlaps and the thesis also aims to develop a consistency of the relation of different tools to avoid confusion thereby bringing in new concepts in valorization. In addition the current existing guidelines are not compatible for implementation of genome-based technologies; therefore the thesis will also aim to contribute to the development of the European best practice guidelines in this field (through PHGEN II). The objectives of the thesis can be summarized as follows:

- Identify the reason behind the backlog or bottleneck of effective and efficient real time integration of health innovations/interventions (genome-based technologies)

- Address the issue of this bottleneck by proposing a framework covering the whole spectrum of translational research possibly through integration and development of (new) concepts

- Demonstrating reasons why certain tools are not relevant to the framework

- Defining the concept of valorization in public health genomics

- Addressing the confusion behind different concepts in translational research and showing the relations between them including conceptual development of the placement of tools within the translational research pipeline

- Developing the step by step methodology of the developed framework

- Giving examples where the new framework can be implemented

- Contribution to the development of the European best practice guidelines on genome-based information and technologies

Conclusively the thesis aims to develop a conceptual framework for the whole pipeline of translational research using the concept of valorization in public health genomics. 


\section{Overview of Thesis Chapters}

The thesis is divided into 5 sections namely, General Introduction (Section 1), Chapters (Section 2), General Discussion (Section 3), Concluding Remarks and Future Prospects (Section 4) and Miscellaneous (Section 5). With respect to the aims mentioned above in the General Introduction, Section 2 (Chapters) is divided into two parts namely Part A that includes chapters I, II, III and IV which deals with the development of theoretical concepts; and Part B that includes chapters V, VI, VII and VIII which deals with the potential implementation scenarios of the developed framework.

Chapter I deals with setting the stage of the issue and defining the concept of valorization in Public Health Genomics, identifying the bottleneck and addressing it by developing a conceptual framework. Chapter II elaborates on all the widely used concepts of translation and implementation of genomebased health innovations. It clarifies the concepts of different tools and demonstrates the place of different tools within other concepts and clears the confusion. It also demonstrates why certain tools were left out of the developed framework in chapter I as well as why the framework is overarching. Chapter III develops the complete detailed protocol/methodology on how to use the model and addresses related issues. Chapter IV gives the general conclusion of Part $\mathbf{A}$ based on the specific objectives defined. Chapter $\mathbf{V}$ describes the complexity of biology as a result of 'omics' and the introduction of systems biology and its relation to personalized healthcare. It proposes the implementation of the developed framework using an example of a current EU flagship pilot project ICT Future of Medicine (ITFoM). Chapter VI gives a review of literature on Chlamydia trachomatis and its relevance to tubal pathology and infertility and the recent development of the potential of developing a diagnostic kit based on an ongoing series of single nucleotide polymorphisms (SNP) markers. Possibly through an SME the developed kit will supplement traditional tests and the thesis's developed framework is proposed here to address the bottleneck mentioned in Part A with this diagnostic kit. Chapter VII contributes to the development of the European best practice guidelines for quality assurance, provision and use of genome-based information and technologies and clears certain concepts. Here the thesis demonstrates the incorporation of the developed framework in the policy guidelines on the European level. Chapter VIII gives the general conclusions of Part $\mathbf{B}$ based on the specific objectives defined. 


\section{| INTRODUCTION}

Section 3 discusses in detail the conceptual development of the framework and discusses the issues of the previous chapters and conceptualizes beyond the status quo. Section 4 derives and simplifies the conclusions over the complete thesis. Section $\mathbf{5}$ gives the summary of the thesis, the CV of the thesis author as well as list of publications and acknowledgements. 


\section{References}

1. Schmitt FO: Contributions of Molecular Biology to Medicine. Bulletin of the New York Academy of Medicine 1960, 36:725-747.

2. Brownlee M: Biochemistry and molecular cell biology of diabetic complications. Nature 2001, 414:813-819.

3. Ansorge WJ: Next-generation DNA sequencing techniques. New Biotechnology 2009, 25:195202.

4. Brown PO, Botstein D: Exploring the new world of the genome with DNA microarrays. Nature Genetics Supplement 1999, 21:33-37.

5. Drews J: Drug Discovery: A Historical Perspective. Science 2000, 287:1960-1963.

6. Collins FS, Green ED, Guttmacher AE, Guyer MS: A vision for the future of genomics research. A blueprint for the genomic era. Nature 2003, 422:835-847.

7. Green ED, Guyer MS: Charting a course for genomic medicine from base pairs to bedside. Nature 2011, 470:204-212.

8. Kitano H: Computation systems biology. Nature 2002, 420:206-209.

9. Lal JA, Sudbrak R, Lehrach H, Brand A: Functional Dynamics: From Biological Complexity to Translation and Impact in Healthcare Systems. Journal of Computer Science and Systems Biology 2013, 6:88-92.

10. Westerhoff HV, Palsson BO: The evolution of molecular biology into systems biology. Nature Biotechnology 2004, 22:1249-1252.

11. Galas DJ, Hood L: Systems biology and emerging technologies will catalyze the transition from reactive medicine to Predictive, Personalized, Preventive And Participatory (P4) medicine. Interdisciplinary Bio Central 2009, 1:1-4.

12. Weston AD, Hood L: Systems Biology, Proteomics, and the Future of Health Care: Toward Predictive, Preventive, and Personalized Medicine. Journal of Proteome Research 2004, 3:179-196.

13. Sorenson $\mathrm{K}$, Brand $\mathrm{H}$ : Health literacy: the essential catalyst for the responsible and effective translation of genome-based information for the benefit of the population health. Public Health Genomics 2011, 14:195-200. 


\section{| INTRODUCTION}

14. Cesuroglu T, Ommen Bv, Malats N, Sudbrak R, Lehrach H, Brand A: Public health perspective: from personalized medicine to personal health. Personalized Medicine 2012, 9:115-119.

15. Hood L, Friend SH: Predictive, personalized, preventive, participatory (P4) cancer medicine. Nature Reviews Clinical Oncology 2011, 8:184-187.

16. PriceWaterCooper: The new science of personalized medicine: Translating the promise into practice 2009.

17. Fontanarosa PB, CD CDD: Basic science and translational research in JAMA. JAMA 2002, 287:1728.

18. Wiedhaup K, Herben C, Meijer I: Valorization. In Partners in the polder A vision for the life sciences in the Netherlands and the role of public-private partnership. Edited by Laane C, Besteman K; 2009: 221

19. Maggon K: Global Biotechnology Market Intelligence Monograph. In Book Global Biotechnology Market Intelligence Monograph (Editor ed.^eds.), 32 edition. City: Knol Publishing Guild; 2011.

20. McDougall G, Rosamond M: Personalized medicine What it means for patient-centered healthcare and how to address its current challenges. PriceWaterCooper View 2010, 13:60, 64,65 .

21. Strength and Opportunity the landscape of the medical technology, medical biotechnology and industrial biotechnology sectors in the UK. In Book Strength and Opportunity the landscape of the medical technology, medical biotechnology and industrial biotechnology sectors in the UK (Editor ed.^eds.). pp. 11-12, 28, 34, 38, 40. City: HM Government; 2009:11$12,28,34,38,40$.

22. US Biotech Market Analysis. 2010.

23. Geenhuizen Mv: Valorisation of knowledge: preliminary results on valorization paths and obstacles in bringing university knowledge to market. In The Eighteenth Annual High Technology Small Firms Conference; May 27-28; University of Twente, Enschede, the Netherlands. 2010

24. Wit Jd, Dankbaar B, Vissers G: Open Innovation: the New Way of Knowledge Transfer? Journal of Business Chemistry 2007, 4:11-17.

25. Mullins B, Crowe J: Technology Transfer: A Roadmap. In Book Technology Transfer: A Roadmap (Editor ed.^eds.), February edition. City: Association of College and Univeristy Auditors; 1999. 
INTRODUCTION |

26. What is the transfer process? [http://cmsserv.ncat.edu/doclib/DORED,\%200utreach\%20and\%20Technology\%20Transfer\% 20(How\%20To\%20Section)\%20Web\%20site,-5.pdf ]

27. Bozeman B: Technology transfer and public policy: a review of research and theory. Research Policy 2000, 29:627-655.

28. Rappert B, Webster A, Charles D: Making sense of diversity and reluctance: academicindustrial relations and intellectual property. Research Policy 1999, 28:873-889.

29. FDA Drug Development Timeline Infographic [http://www.pkdcure.org/learn/healthcareprofessionals/drug-development-timeline-infographic]

30. Lal JA, Bäumen TSid, Morré SA, Brand A: Public health and valorization of genome-based technologies: a new model. Journal of Translational Medicine 2011, 9.

31. Health Needs Assessment - A Practical Guide. In Book Health Needs Assessment - A Practical Guide (Editor ed.^eds.). City: Health Development Agency; 2005.

32. Rosenköetter N, Vondeling H, Blancquaert I, Mekel OCL, Kristensen FB, Brand A: The Contribution of Health Technology Assessment, Health Needs Assessment, and Health Impact Assessment to the Assessment and Translation of Technologies in the Field of Public Health Genomics. Public Health Genomics 2011, 14:46-47.

33. Wright J, Williams R, Wilkinson JR: Development and importance of health needs assessment. British Medical Journal 1998, 316:1310-1313.

34. Gray JAM: Evidence-Based Healthcare. How to Make Health Policy and Management Decisions. New York: Churchill Livingestone; 1996.

35. Health Technology Assessment (HTA) [http://www.eunethta.eu/about-us/faq\#t287n73]

36. Battista RN: Towards a paradigm for technology assessment. In The Scientific Basis of Health Services. Edited by Peckham M, Smith R. London: BMJ Publishing Group; 1996: 11-18

37. Garrido MV, Kristensen FB, Nielsen CP, Busse R: Health Technology Assessment and Health Policy Making Current status, challenges and potential. In Book Health Technology Assessment and Health Policy Making Current status, challenges and potential. 2008.

38. Robert G, Stevens A, Gabbay J: 'Early warning systems' for identifying new healthcare technologies. Health Technology Assessment 1999, 3:100-108.

39. Douma KF, Karsenberg K, Hummel MJ, Bueno-de-Mesquita JM, Harten WHv: Methodology of constructive technology assessment in health care. International Journal of Technology Assessment in Health Care 2007, 23:162-168. 


\section{| INTRODUCTION}

40. Stevens A, Milne R, Burls A: Health technology assessment: history and demand. Journal of Public Health Medicine 2003, 25:98-100.

41. Battista RN, Hodge MJ: The "natural history" of health technology assessment. International Journal of Technology Assessment in Health Care 2009, 25:281-284.

42. Health Technology Assessment

Process

[http://www.cgh.uottawa.ca/WHOCC/projects/eo_toolkit/hta.htm]

43. Gothenburg Consensus Paper. Health Impact Assessment. Main concepts and suggested approach. In Book Gothenburg Consensus Paper. Health Impact Assessment. Main concepts and suggested approach (Editor ed.^eds.). City: HIA Gateway, West Midlands Public health Observatory; 1999.

44. Parry J, Stevens A: Prospective health impact assessment: pitfalls, problems, and possible ways forward. British Medical Journal 2001, 323:1177-1182.

45. Harris-Roxas B, Harris E: Differing forms, differing purposes: A typology of health impact assessment. Environmental Impact Assessment Review 2011, 31:396-401.

46. Sanderson S, Zimmern R, Kroese M, Higgins J, Patch C, Emery J: How can the evaluation of genetic tests be enhanced? Lessons learned from the ACCE framework and evaluating tests in the United Kingdom. Genetics in Medicine 2005, 7:495.

47. ACCE Model Process for Evaluating Genetic Tests [http://www.cdc.gov/genomics/gtesting/ACCE/index.htm]

48. Evaluation of Genomic Applications in Practice and Prevention (EGAPP) [http://www.egappreviews.org/about.htm]

49. EGAPP: Understanding EGAPP: Four Steps of the EGAPP Working Group Review Process. In Book Understanding EGAPP: Four Steps of the EGAPP Working Group Review Process (Editor ed.^eds.). pp. 2-7. City; 2008:2-7.

50. Khoury MJ, Gwinn M, Yoon PW, Dowling N, Moore CA, Bradley L: The continuum of translation research in genomic medicine: how can we accelerate the appropriate integration of human genome discoveries into health care and disease prevention. Genetics in Medicine 2007, 9:665.

51. Burke W, Khoury MJ, Stewart A, Zimmern RL: The path from genome based research to population health: Development of an international public health genomics network. Genetics in Medicine 2006, 8:453. 
52. Beskow LM, Khoury MJ, Baker TG, Thrasher JF: The integration of Genomics into public health research, policy and practice in the United States. Community Genetics 2001, 4:4.

53. Peng W: Trends in biotech literature 2009. Nature Biotechnology 2009, 28:887.

54. Catalogue of Biotechnology Patents in the Mercosur. BIOTECHSUR 2008.

55. Snyder M, Cook-Deegan B: DNA Patent Database Statistics: 2010. DNA Patent Database 2011.

56. Berwick DM: Dissseminating Innovations in Health Care. Journal of the Amercian Medical Association 2003, 289:1969-1970.

57. Syurina EV, Bäumen TSid, Brand A, Ambrosino E, Feron FJM: Concepts for the translation of genome-based innovations into public health: a comprehensive overview. Personalized Medicine 2013, 10:163-176. 



\section{Section 2}

\section{Chapters}

PART A

Chapter I

Chapter II

Chapter III

Chapter IV

PART B

Chapter V

Chapter VI

Chapter VII

Chapter VIII 

PART A

Establishment of Theoretical Concepts 


\section{Specific Aims}

This part of the thesis deals with developing the theoretical foundations based on the problem statement of the bottleneck of the real-time effective and efficient integration of genome-based technologies into healthcare as stated in the introduction chapter. The part however first deals with identifying and addressing the problem and building around theoretical concepts and the protocol to execute the solution as well as defending the solution's overarching reach. In relation to the general objectives stated in the introduction chapter, this part relates to the following mentioned general objectives:

- Identify the reason behind the backlog or bottleneck of effective and efficient real time integration of health innovations/interventions (genome-based technologies)

- Address the issue of this bottleneck by proposing a framework covering the whole spectrum of translational research possibly through integration and development of (new) concepts

- Demonstrating reasons why certain tools are not relevant to the framework

- Defining the concept of valorization in public health genomics

- Addressing the confusion behind different concepts in translational research and showing the relations between them including conceptual development of the placement of tools within the translational research pipeline

- Developing the step by step methodology of the developed framework

Deriving from these general objectives, the specific aims of Part A are as follows:

- Chapter I

- Presenting the problem statement of the bottleneck of integration of genome-based technologies into healthcare

- Identifying the rationale behind the problem statement

- Introducing tools to resolve the problem statement

- Defining valorization in public health genomics

- Developing the framework to close the gap - the LAL model

- Chapter II

- Elaborating on various concepts used in the field and arguing the exclusion/inclusion of them with respect to the developed framework 
- Resolving the issue of overlapping tools and defining them with relation to each other

- Argumentation why the solution framework is the answer

- Chapter III

- Elaboration on the defined steps of the solution framework

- Addressing issues still to be resolved

- Chapter IV

Deriving conclusions based on the specific objectives 

CHAPTER I

PUBLIC HEALTH AND VALORIZATION OF GENOME-BASED TECHNOLOGIES: A NEW MODEL

Published as:

Lal JA, Schulte in den Bäumen T, Morré SA, Brand A. Journal of Translational Medicine 2011, 9:207. 
Background: The success rate of timely translation of genome-based technologies to commercially feasible products/services with applicability in health care systems is significantly low. We identified both industry and scientists neglect health policy aspects when commercializing their technology, more specifically, Public Health Assessment Tools (PHAT) and early on involvement of decision makers through which market authorization and reimbursements are dependent. While Technology Transfer (TT) aims to facilitate translation of ideas into products, Health Technology Assessment, one component of PHAT, for example, facilitates translation of products/processes into healthcare services and eventually comes up with recommendations for decision makers. We aim to propose a new model of valorization to optimize integration of genome-based technologies into the healthcare system.

Methods: The method used to develop our model is an adapted version of the Fish Trap Model and the Basic Design Cycle.

Results: We found although different, similarities exist between TT and PHAT. Realizing the potential of being mutually beneficial justified our proposal of their relative parallel initiation. We observed that the Public Health Genomics Wheel should be included in this relative parallel activity to ensure, all societal/policy aspects are dealt with preemptively by both stakeholders. On further analysis, we found out this whole process is dependent on the Value of Information. As a result, we present our LAL (Learning-Adapting-Leveling) model, which proposes, based on market demand; TT and PHAT by consultation/bi-lateral communication should advocate for relevant technologies. This can be achieved by public-private partnerships (PPPs). These widely defined PPPs create the innovation network, which is a developing, consultative/collaborative-networking platform between TT and PHAT. This network has iterations and requires learning, assimilating and using knowledge developed and is called absorption capacity. We hypothesize that the higher absorption capacity, higher success possibility. Our model however does not address the phasing out of technology although we believe the same model can be used to simultaneously phase out a technology 
Conclusions: This model proposes to facilitate optimization/decrease the timeframe of integration in healthcare. It also helps industry and researchers to come to a strategic decision at an early stage, about technology being developed thus, saving on resources, hence minimizing failures.

Keywords: Technology Transfer, Health Technology Assessment, Public Health Genomics, Health Needs Assessment, Health Impact Assessment, Valorization, Translational Research, Healthcare, Health Policy, Genomics 


\section{| CHAPTER |}

\section{Background}

Over time we have seen enormous transition of genome-based/life science research from the lab to products and technologies [1-3] on the market [3-6] as a result of knowledge valorization and spin-offs $[2,3,7,8]$. This can be attributed to the concept of translational research, which is the effective translation of new knowledge, mechanisms and techniques generated by advances in basic science research into new approaches for prevention, diagnosis and treatment of disease essential for improving health [9]. However, we notice that the timely translation of genome-based technologies to commercially feasible products with practical applicability or direct implementation in health care systems is quite low [10]. This is evident by the large amount of data present in literature [11], patents $[12,13]$ and the market $[3,5]$ compared to what actually is being used in hospitals $[10,14]$. We identify, based on our experience as well as derivatively, three phases of translation. The first phase includes translation from lab to industrial application (see T1 of Khoury et al.) [15], the second phase being from industry to market penetration [16] and the third phase being, shift from the market to integration in health policy (see T3 of Khoury et al.) [15]. We believe both academia and industry focus only on one or maximum two of the three translational phases.

In addition, methodologies in place for translation generally focus on the first two phases or the last phase and as per our knowledge we have not seen a combination of the three, overlaps or jumps in general. For example, Technology Transfer (TT) mainly addresses the first two phases mentioned above. TT is seen as an activity of the migration of academic discoveries to useful application in the development of marketable products or processes [17]. TT can involve several steps from organization to organization and can have separate offices specialized in this activity. For example, universities have a TT office or valorization center responsible for $\mathrm{TT}$ activities of university research. Basically, the $\Pi$ activity initiates from an invention of an innovative idea, through the development of the idea into a pilot to the creation of the technology based on the idea followed by patenting the technology in question and ends after the technology maturation process. The maturation process involves the return on investment and exit strategy of a company with respect to the technology concerned. A simplistic example can be seen from Figure 1 below [18]. TT is the most widely used activity [1] in business development of academic research and spin-offs [19]. It should be noted here however, $\Pi$ can be considered as an activity, 
methodology, tool or technique depending on the user. However from the objective of our paper, we will consider this as an activity.

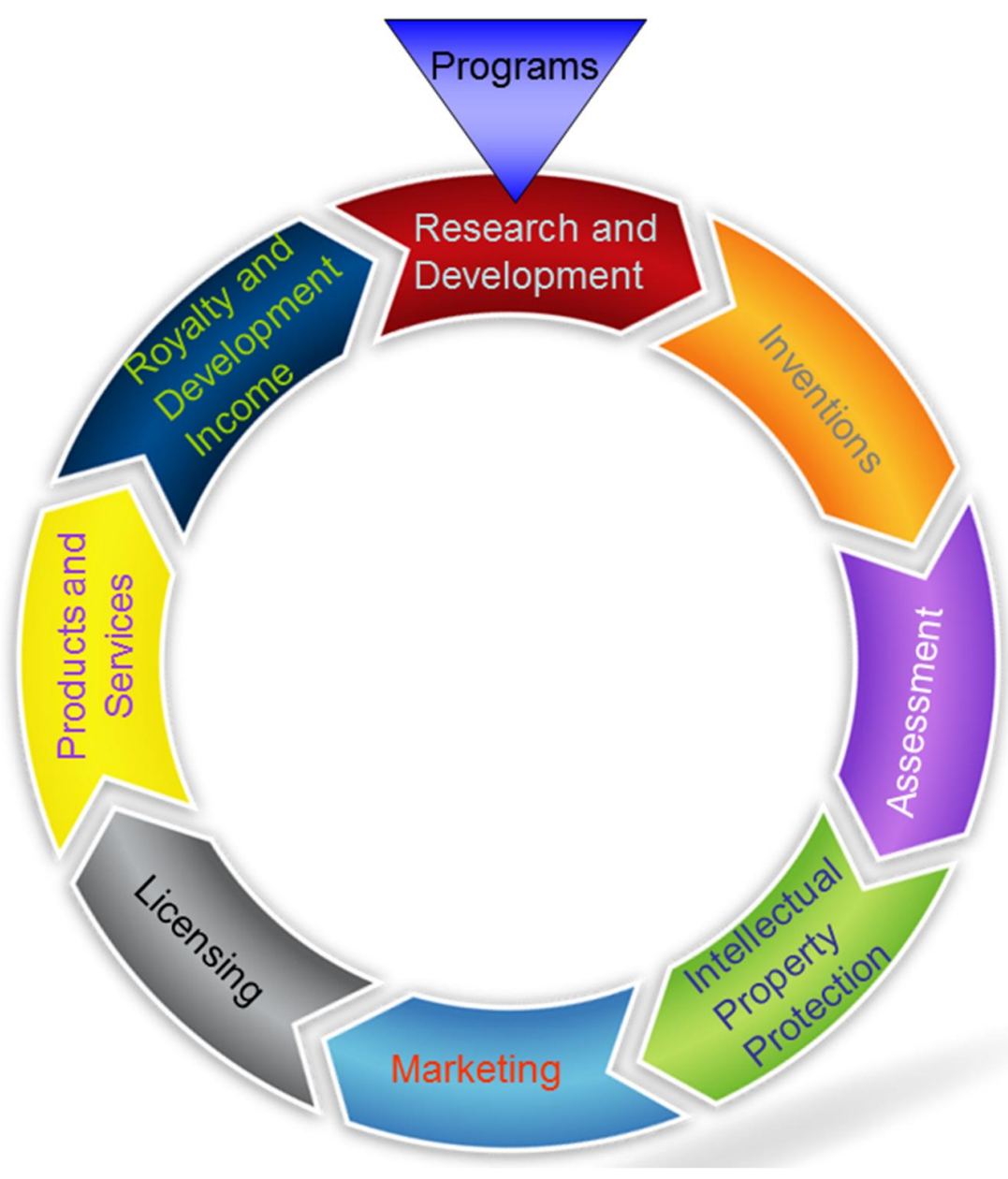

Figure 1: The Technology Transfer Process. The basics include the start from research and development based on market pull or push, successfully inventing a product based on that research, patenting the product, thereby marketing and licensing and going through the technology maturation process. Taken from the North Carolina Agricultural and Technical State University [18].

The most common procedures used by decision makers (DM) and health professionals in phase three mentioned above involve the traditional public health evaluation instruments [20], which include Health Needs Assessment (HNA), Health Technology Assessment (HTA) and Health Impact Assessment (HIA). HNA is a systematic method of reviewing the health issues facing a population, leading to agreed priorities and resource allocation that will improve health and reduce inequalities [20]. Correspondingly, HTA is a multidisciplinary process that summarizes information about the medical, social, economic and ethical issues related to the use of a health technology in a systematic, transparent, unbiased, robust 


\section{| CHAPTER |}

manner. Its aim is to inform the formulation of safe, effective, health policies that are patient focused and seek to achieve best value [21]. This implies to interventions as well as HTA's involvement in appraisals. Thus, HTA is a powerful tool to inform policy making. According to the World Health Organization, HIA is a combination of procedures, methods and tools by which a policy, program, or project may be judged as to its potential effects on the health of a population, and the distribution of those effects within the population [22]. To summarize, HTA evaluates the performance of health care technologies, HIA assesses the effects of policies, programs or projects on the population's health, and HNA identifies health priorities for a given population [20]. For our convenience, we will use the term Public Health Assessment Tools (PHAT) for the collective reference to HNA, HTA and HIA in the text.

It becomes apparent from above, that the translational phases 1-2 and 3 mentioned above are investigated by two separate entities, namely, the academia-industry infrastructure [23] and the governmental bodies respectively. This is the key reason why the two operate as separate functions. While $\Pi$ aims to facilitate the translation of ideas into products, HTA for example, assesses the translation of products and services into healthcare. As per content, they both seem inseparable in the order stated, however to our knowledge have never been connected. As a result, there is a backlog of relevant technologies to be integrated in Public Health and healthcare in general in a timely as well as effective and efficient manner $[10,24,25]$. Even by the time a technology is introduced in the health care system, it is in a way deemed redundant as more effective and efficient technologies become available in the market. Redundancy is from the perspective of the potential of existing technologies on the market compared to the previously introduced one in the hospital. Efficiency measures whether healthcare resources are being used to get the best value for money [26]. Therefore, efficiency can be seen in terms of added value, speed and cost, although costs can vary. According to Ostrower [27], effectiveness has several varying definitions but one of the common ones used by institutions includes the component of 'having an impact'. On the other hand (clinical) effectiveness is the extent to which specific (clinical) interventions do what they are intended to do, i.e. maintain and improve the health of patients securing the greatest possible health gain from the available resources [28]. In other words, effectiveness and also in our case, refers to the achievement of the overall goal (the effect). We identify the non-synergy between TT and PHAT, as the bottleneck of technological integration in the health care.

This brings in the new field of Public Health Genomics, which is the responsible and effective translation of genome-based knowledge and technologies into public policy and health services for the benefit of 
population health [29]. It is an emerging field with increased demands of integration of genome-based innovations i.e. genome-based knowledge and technologies, into Public Health due to the prior's increasing value and relevance in the latter. Within the concept of Public Health Genomics and keeping in mind the bottleneck mentioned above, we aim to propose a new model to facilitate valorization of genome-based technologies into the healthcare system in real time.

The term valorization can vary depending upon the user (say either the economist or entrepreneur). According to the Merriam-Webster online dictionary [30], one definition of valorize states 'to assign value or merit to'. On the other hand, the term valorization can be defined as the transformation of knowledge into concrete new products, services and processes [31]. Deriving from these two definitions and keeping in mind the definition of Public Health Genomics, valorization in Public Health Genomics can be seen as the 'process of realization' of relevant added value 'bioproducts' in the domain of Public Health for benefit of the population and healthcare systems. Here we consider realization in terms of understanding the importance, impact or potential benefit and implementing it. The term bioproduct may have varying definitions $[32,33]$, here again based on the user or source. According to the working definition submitted to the British Columbia Bioproducts Working Group [34] with the background of agro-forestry, bioproducts are sustainable, environmentally friendly novel products, or products satisfying novel applications and generated from renewable (living) bioresources based on technologically advanced eco-efficient conversion processes. Within our scope of usage focusing on integration of genome-based technologies/life sciences into healthcare, we define bioproducts as sustainable novel products or products satisfying novel health applications derived or inspired from genome-based information or technologies. We define a product as a system, device, technique or process or application. Genome-based technologies encompass all -omics initially deriving from the (Human) Genome. 


\section{Methods}

The methodology we used to develop the model is based on the Basic Design Cycle [35] and the Fish Trap model [36] as can be seen from Figure 2 and 3 respectively. The general methodologies mentioned above are used mainly in designing products for commercial exploitation. In our case, the methodologies have been adapted to fit our purpose, which can be seen in Figure 4 below. We use this adapted design technique to address a Public Health and health policy issue, the integration of genome-based technologies into healthcare in the concept of a new model. In the subsequent paragraphs we briefly explain the two models from which our methodology derived from followed by the derived methodology itself.

The Basic Design Cycle (BDC) is stated to be the most fundamental model of designing. The intended behavior or function of the product is the start of product design as can be seen from Figure 2. Broad statements on the function/properties (use) are made in order for the designer to know what needs to be designed. In the analysis phase the problems surrounding a new product idea are identified (also called the problem statement), thereby criteria are formulated from general to specific ones over iterations, which the solution should satisfy. The next step is the development of a provisional design via the synthesis phase (combining things) [35]. This phase is based on human creativity and is considered the moment of externalization and description of an idea on any form. The next phase is simulation phase, which is considered a deductive process. Simulation in essence is forming an image of the behavior and properties of the designed product through testing, reasoning, etc. and results in anticipation of the real properties or conditional predictions for the new product. The next phase is the evaluation in which the value of the design

Figure 2: The basic design cycle. The most basic design methodology used. All design problems have one way or the other went through this design directly/indirectly. See text for description. Taken from Roozenburg et al. [35].

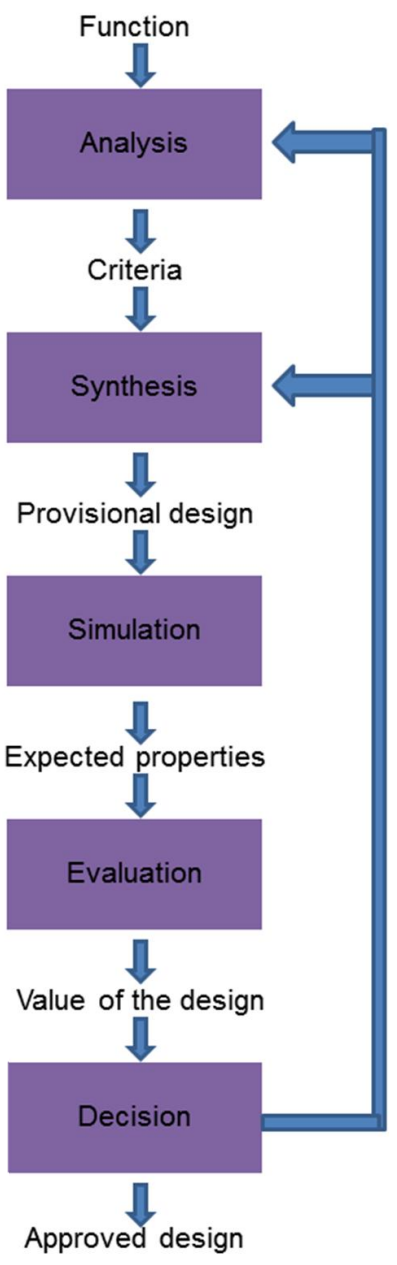

is established. This is based on 
comparison of previously mentioned set specific criteria (see Figure 2) and actual properties in the design. The last phase is the decision phase whether to continue or reconsider. There is the possibility that the designer may need to return to the analysis or synthesis with subsequent iterations and feedback loops [35].

The Fish Trap Model (FTM) (can be considered to be called as to catch a final solution) is a systematic process of designing a product form. As can be seen in the Figure 3, the fish trap is illustrated in two ways, (Figure $3 \mathrm{~A}$ ) to visualize the divergence and convergence as well as to show the occurrence of various solution types at each level [36]. Once possible variants are created through curiosity/possibilities, they are categorized according to solution type. Thereafter, one or more variants are chosen to be developed into a concept, which indicates a specific solution type. Again, from here more concrete solutions are derived from the chosen variants thus diverging again. This narrows down to one or few options to go forth with [36].

As can be seen from Figure 3B, the FTM is represented in a step by step or phase format. Since the model pushes the designer to discover alternatives on 3 levels, namely topological, typological and morphological, it is considered a systematic process as can be seen in the figure. Following step by step Figure $3 \mathrm{~B}$, in order to develop the 'structural concept' the 'basic structure' functional components need to be defined in advance through 'visualization of context'. One can develop or 'generate' multiple variants with these components that may differ in their respective spatial placement. These divergent are then clustered (convergence) or 'categorize' and a representative variant are selected to be developed into a 'structural concept'. Again, selection of these structural concepts will be based on evaluation criteria and need to be put into context. For the development of a 'formal concept', one or more structural concepts are taken [36]. From these selected structural concepts, geometric constructions via sketching are made leading to different classes of form (generate step). These sketches are evaluated based on their viability and categorized into groups based on form, and improvements can be still made and evaluated. Thereafter, promising candidates are further developed into one or more 'formal concepts'. Similar to above, further materialization of one or more formal concepts is done with a diverging exploration process again looking at a detailed level at the solution [36]. 
A

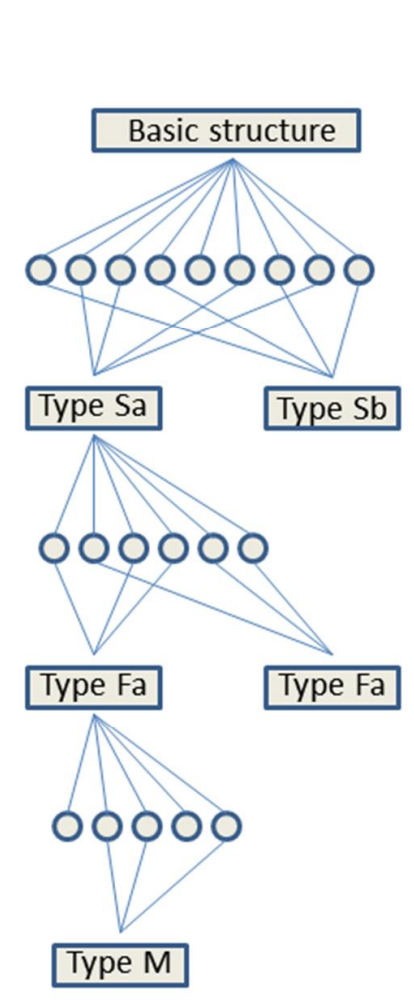

B

Visualization of context

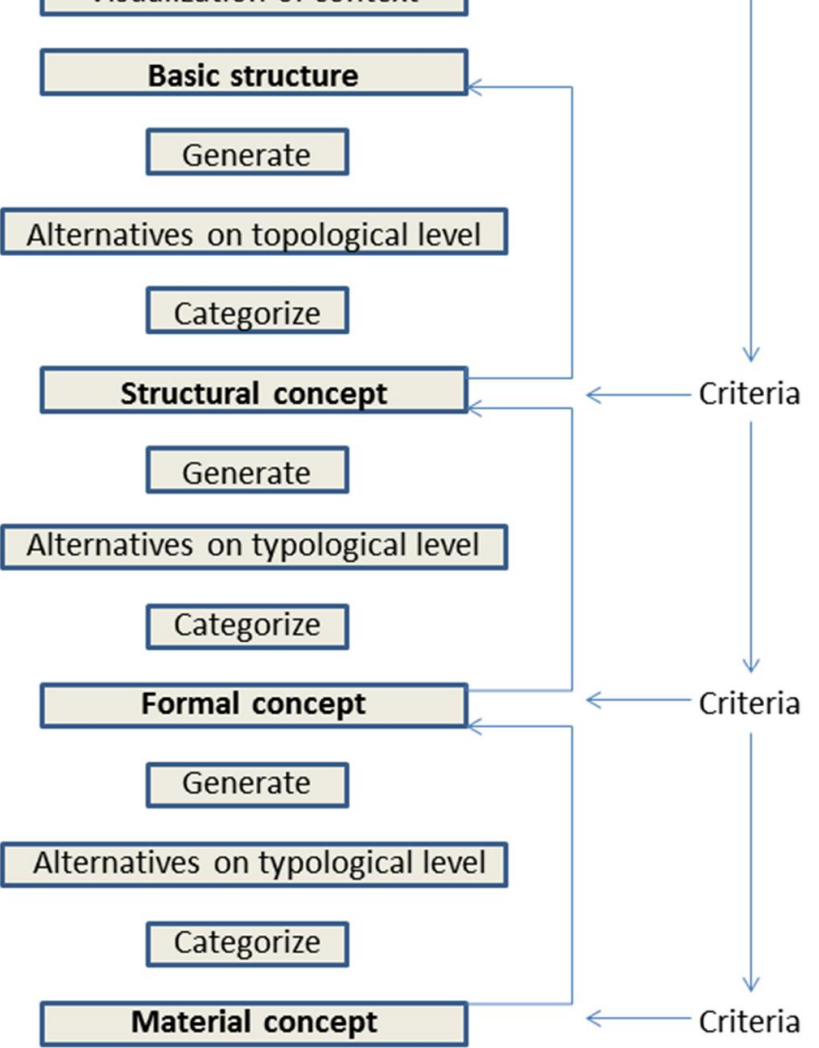

Figure 3: The fish trap model. (A) The convergence-divergence illustration. 'Sa and Sb' refer to structural concept a and structural concept $\mathrm{b}$ respectively. Similarly, ' $\mathrm{Fa}$ and $\mathrm{Fb}$ ' refer to formal concept $\mathrm{a}$ and formal concept $\mathrm{b}$ respectively. ' $\mathrm{M}$ ' refers to material concept. (B) the phase or step-wise illustration of the fish trap model. See text for description. Taken from the Delft Design guide [36].

Our methodology was adapted from the above two mentioned models. As can be seen in Figure 4 the first phase is the problem phase derived from the concept of the 'problem statement' of the analysis phase of the Basic Design Cycle (BDC). In our model the problem phase includes both stating the problem (statement) of the BDC as well as identification of the intended behavior or function of the product which in our case is a framework. This phase not only deals with identifying the problem which needs to be solved but as well as understanding it. As a result the intended function of the final solution is cleared from the beginning.

The next step in our methodology is the analysis phase, which is derived partly from the remainder of the analysis phase in the BDC and the structural concept phase of the Fish Trap Model (FTM). From the latter, the basic functional components are taken into our analysis phase. In other words, we look at the 
problem and its potential components and factors in more detail and analyze the data. Additionally, from the BDC concept, our analysis phase also includes developing criteria for the analysis and can include possible iterations and feedback.
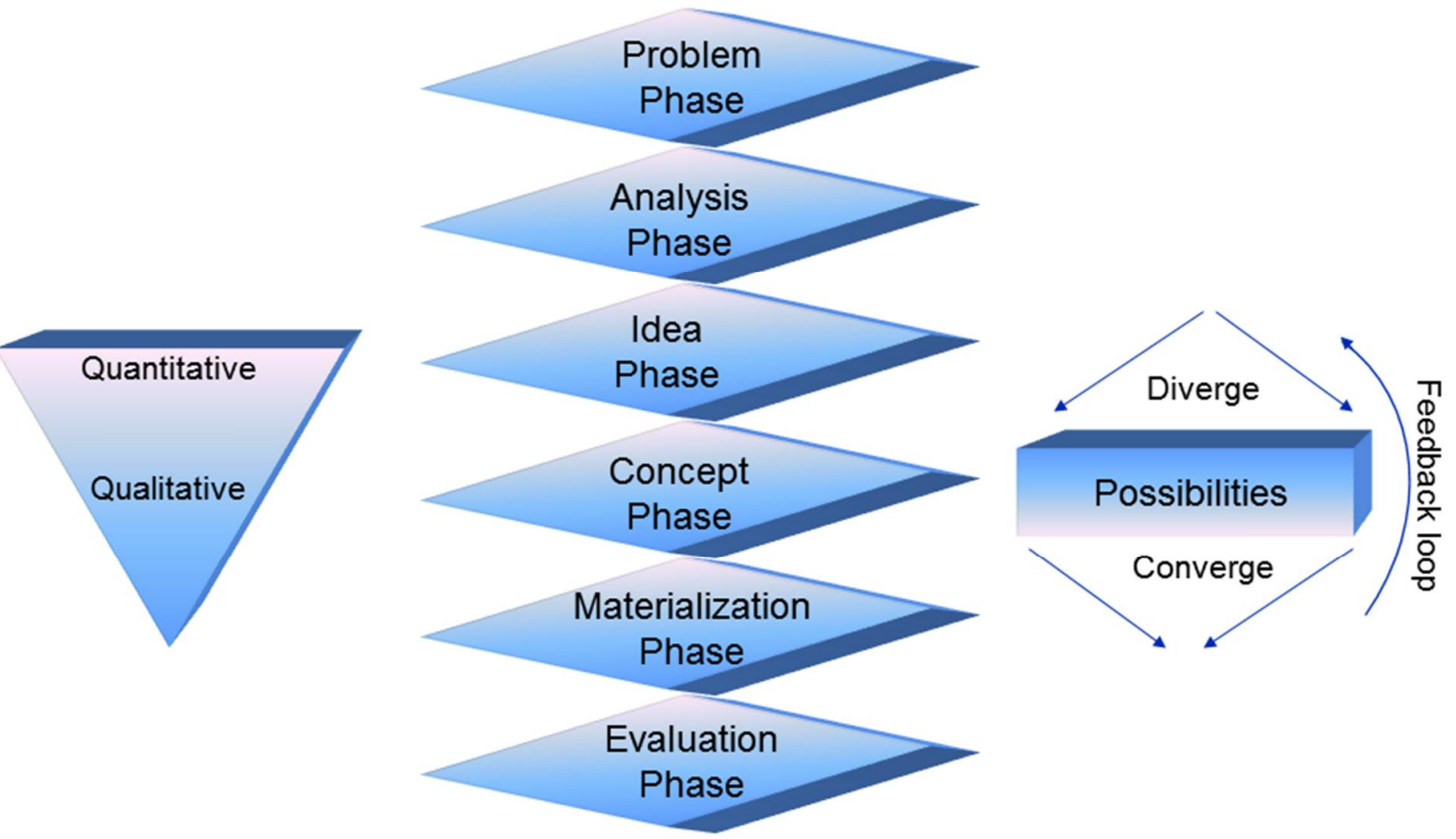

Figure 4: The methodology adapted from the Fish Trap Model and the Basic Design Cycle. The first phase is called the 'Problem phase'. This phase deals with identifying the problem, which needs to be solved, and understanding it. The next phase is the 'Analysis phase'. In this phase we look at the problem in detail and analyze relevant data per se. Next comes the 'Idea phase'. This phase derives possible solutions based on the analysis phase coming up with the possible options, directions or ideas to proceed with and can overlap with the analysis phase. Following the idea phase is the 'Concept Phase'. From the list of options generated, based on merits, one or few are selected to work on further detail or conceptualize it. Thereafter the conceptualization, the product is materialized or brought forth and implemented. This is followed by the evaluation of the product. The whole process can have iterations and moves from a large set of options and narrows down to a single idea to be implemented. Adapted from the Basic Design Cycle [35] and the Fish Trap Model [36].

As a result of the analysis phase several options are generated/developed to go forward with, which we call the idea phase and is the next step in our methodology. The components of this phase are derived from the synthesis phase of the BDC, which is based on human creativity (and describes the idea on any form) and the divergence concept (several possibilities) of the FTM. Also, from the FTM, the basic functional components previously defined in our analysis phase are developed into multiple variants and clustered forming a structural concept. This step is considered the externalization of several relevant ideas. 


\section{| CHAPTER |}

The concept phase is the follow-up step of the idea phase in our methodology and is based on the simulation step of the BDC to develop the provisional design and is a deductive process as one or more options are chosen from a list (in the idea phase) based on the context it can solve. Also, the concept phase influentially derives from the formal concept step of the FTM, which include assessing one or more structural concepts from our idea phase as well as geometric sketches. From these sketches through iterations and feedback loops one final version is selected.

The above mentioned steps in our adapted methodology (see Figure 4) in line with the BDC and the FTM, initially quantify the data down to a single qualitative product or in our case, a model. This is achieved through iterations and feedback loop, which include diverging and converging of data or components. In other words development initiates with several ideas and through the process and feasibility analysis narrows down from a quantity of data to one final option good in quality. The scope of our methodology includes and ends at the concept phase since based on the fact the model we propose is a theoretical framework. Nonetheless, validation or proof of principle and evaluation are logically the next steps although not within the scope of this paper. Therefore we keep these two points in the to-be final two steps of our methodology mentioned below.

Materialization phase will be the next step of our methodology although not utilized in the paper. This is derived directly from the materialization phase of the FTM and may include diverging exploration if necessary. The implementation of the product or in our case the model/framework is considered the completion of this phase. The final to-be phase is the evaluation phase, which is a direct derivation of the decision phase of the BDC with a modified approach to evaluate rather than to come to a decision. However this evaluation can lead to further adaption of the model. 
On further analysis, we identified that the mainly used activity in transfer of academic knowledge to industry/market is TT $[1,19]$. On the other hand, the system used by decision makers to come to a decision regarding implementation of technologies in the healthcare system is the PHAT. For simplicity, we will take one example of the PHAT, i.e. HTA with references to other components when seemed appropriate. As known HTA is initiated by the need and identification of technologies which can reduce the burden of illness and through its steps, prioritize those with importance and relevance $[37,38]$ and eventually come up with recommendations for DMs. Taking into account the HTA process [37] as can be seen in Figure 5 below, it has several steps. The TT activity however, similar to HTA in its complexity of dynamic steps, varies from organization to organization. For our case we will use the simple example, given by North Carolina Agricultural and Technical State University, [18] as shown in Figure 1 above. As we can see from the Figures 1 and 5, there are quite some similarities or overlaps between the two different approaches as well as per definitions of the two seem inseparable in the order stated.

However, after comparing Figure 1 and 5 above, it seems that TT should move in parallel with HTA rather than one after the other. Below are suggested relations between the two:

1. HNA/HTA initiates with the identification of current health needs and prioritizes accordingly. On the other hand, TT, kick-starts with research and development, which in part is motivated by the current needs in the market (market pull) or creates a need based on scientific data (market push). It should be noted here that literature suggests a significantly large amount of scientific data relevant and applicable to health [11] which is not implemented in the same [10], therefore justifies market push in health and medicine. The key word here being 'need' is the common ground of motivation for both TT and HNA/HTA initiation, suggesting the reason for both of them to start in parallel unlike what their respective definitions suggestion of one after the other. In other words, HNA and HTA identify the gaps in the needs of healthcare technology and its subsequent requirement of investigation respectively, and TT capitalizes on this need.

2. It should be stressed that consultation with external experts form a core component of HTA during the prioritizing of relevant technologies. Generally speaking, experts in the field develop the technology in the TT process, and with regard to medical technologies, take feedback from the end-consumers. 


\section{CHAPTER I}

3. The core component of HTA is the assessment phase (including the economic, ethical, legal and social implications) of the technology in question and can also include Horizon Scanning (can be considered part of HNA and early stages of HTA) [39]. Horizon Scanning is defined as a systematic process for objectively evaluating the status of potential benefits of foreseeable technological developments based on contemporary research and evidence [40]. It also implies that it may be used as a type of SWOT (Strengths, Weaknesses, Opportunities and Threats) analysis [40]. Assessment of the feasibility of the invented technology is a given in TT before patenting or placing it in the market and this generally includes SWOT analysis. This also brings forth the HIA, where the impact of potential technology to be used is assessed, which also related to the TT's strategy before introducing the technology concerned in the market. It seems logical that TT should encompass Horizon Scanning within the aspect of HNA and HTA.

4. Another point is that both fields identify related stakeholders during the process.

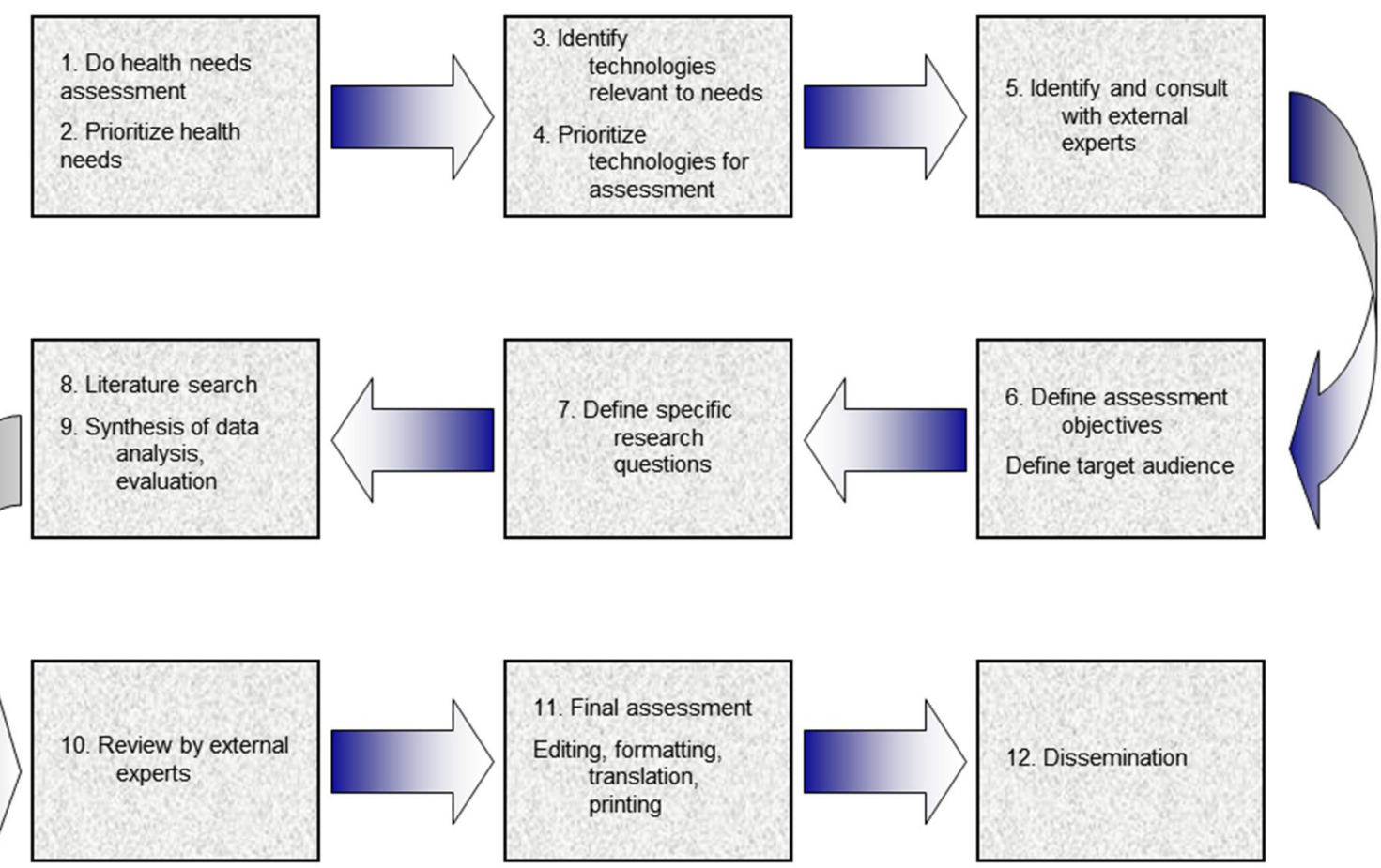

Figure 5: A general outline of the HTA procedure. The picture displays how HTA investigation initiates and the different steps involved followed by dissemination or recommendation to Government officials, for example whether or not to go forward with the technology in question in health policy implementation. Taken from the Canadian Coordinating Office for HTA [37]. 
It is not of the reasons above that the two (PHAT and TT) should start off together. Rather the essence of the story lies in the fact that both separate fields can and should benefit from each other and en-route streamline the integration process in order to properly address the whole innovation pipeline. Based on the authors' experience working with, in and around the Biotech industry, the bottleneck of technology integration is rooted in the fact that the industry neglects the policy aspects [41] (can include but not limited to market authorization, reimbursement and market penetrance) of technology which is an important factor in determining technology use in Public Health and healthcare at the end of the technology maturation process. Similarly, DMs only assess existing mature technologies to cover the aspects of current need, which results in the untimely introduction of relevant or upcoming technologies that can solve the 'need' issue more effectively and efficiently. With this flow of thought, the above mentioned points which suggest the anonymous inter-relations between TT and PHAT bring forth a new window of opportunity to use these pre-existing respective setups to expand the bottleneck for sustained flow of technological integration in the healthcare system through simultaneous initiation of these methods rather than one after the other.

Based on the above, our model should include relative parallel initiation of TT and PHAT with a sustainable infrastructure or network for the two to communicate bi-laterally and mutually benefit. On further research, we found that the Public Health Genomics Wheel (PHGW) [42], which describes Public Health components or areas that can be addressed by genome-based information and technologies, seems suitable to fit in our model. Furthermore, we identified that the Value of Information (VOI), the amount a person is willing to pay to come to a decision on a subject [43], seems appropriate for our model.

As a result of our investigation, we present our model which we call the 'LAL Model' as can be seen in Figure 6 below and stands for 'Learning-Adapting-Leveling'. As we know, TT is driven by either market pull or market push and PHAT is driven by market pull. A technology (or market) push implies that a new invention is pushed through research and development, production and sales functions onto the market without proper consideration of whether or not it satisfies a user need [44]. In contrast, an innovation based upon market pull has been developed by the research and development function in response to an identified market need [44]. Also it is known that market push can lead to market pull and vice-versa. It should be noted here when talking in terms of PHAT market pull is not motivated due to commercialization although $\Pi T$ may recognize it in that way, but rather by requirement or health need. 


\section{| CHAPTER |}

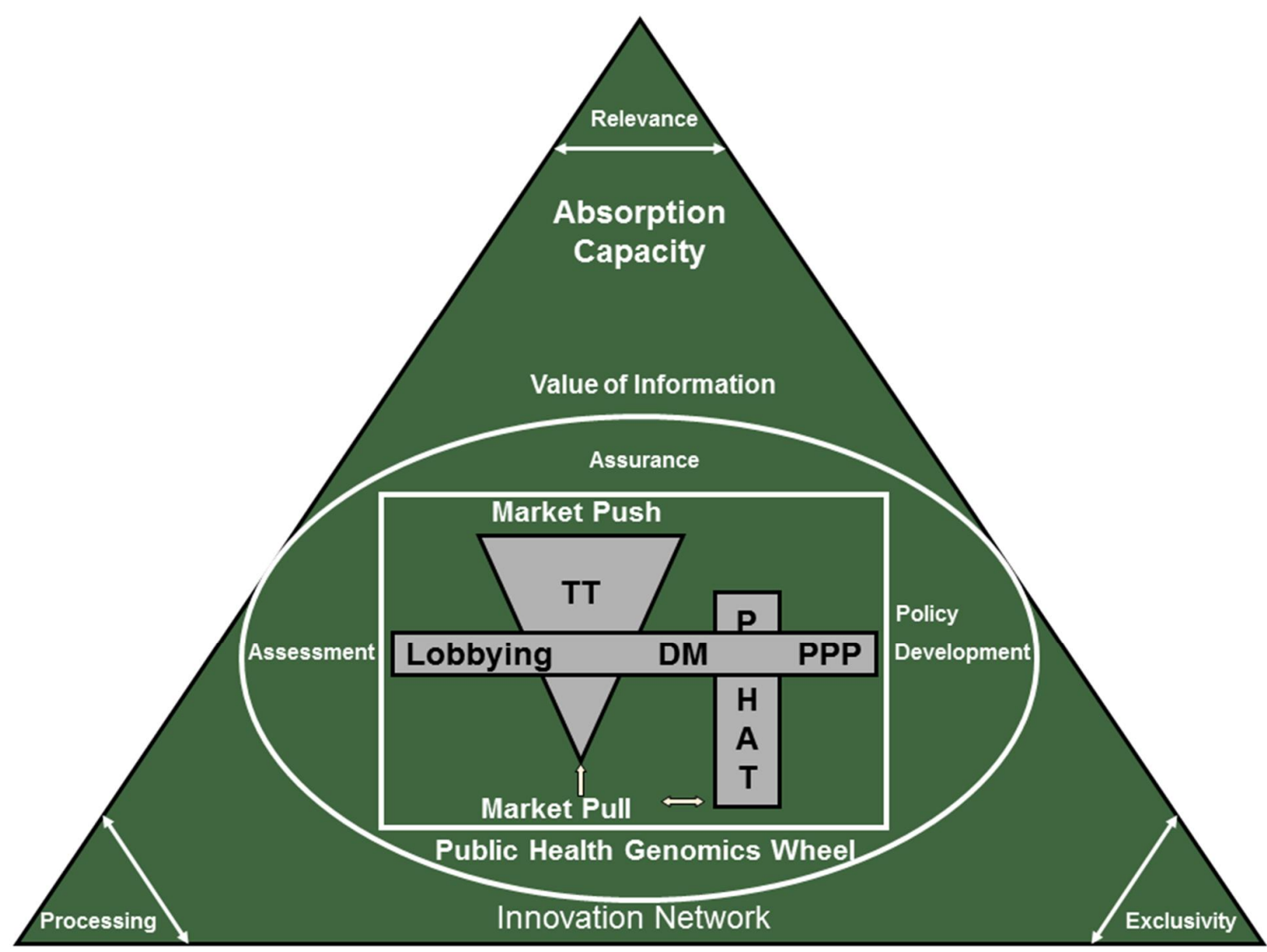

Figure 6: The LAL Model - Learning Adapting Leveling. The model's core consists of two components, the TT activity and the PHAT process (used by DMs). This model proposes bi-lateral communication, collaboration and advocating for relevant technologies during and between the (early-mid) TT activity and PHAT process both of which are driven by market pull/push (common denominator). This is achieved possibly through PPPs. The two components/entities (TT \& PHAT) use the PHGW as a reference point to see that all policy and societal aspects are covered. This ongoing interaction through PPPs develops the innovation network for the current and future collaborations which is a network or platform through which common goals can be achieved. This innovation network is dependent on the VOI, i.e. the actual relevance (including added value) of the technology in question, the exclusivity (patent, license, etc.) of the technology to be integrated including possible hindering factors as a result, and the processing ability or understandability of user (say health professional or doctor, etc.) with respect to the technology and its impact. Over the process, the two core components (TT and PHAT), learn and adapt from each other within the innovation network and level out differences in approach and concept through PPPs (in essence learning-adapting-leveling) through feedback loops and iterations. This can be achieved over multiple or repetitive PPPs. Each network has a threshold of optimal capacity for such a learning curve, which we call the absorption capacity. The model indicates the right balance between profit and timely interventions.

However, the (market) pull or need is the common ground which fuels both PHAT (in particular HNA/HTA) and TT respectively. When we talk about TT, it moves from quantitative to qualitative output. In other words, research and development initiates with several ideas and through the process and feasibility analysis, quantifies down to a single qualitative product to be commercialized. This is 
represented in our Figure 6 as $\pi$ being an inverse triangle. What also can be noticed is that the TT triangle and PHAT bar are standing in parallel, with the prior starting a bit earlier than the latter. TT identifies possibilities of commercialization via market pull/push based on sound scientific knowledge. The initial stages of $T T$ start in the lab with research and development. At this stage, the research is motivated on market dynamics and cannot clearly identify with the PHAT's Health Needs Assessment; hence the reason of this kind of leveling or kick-off.

Based on the previous common grounds identified in the two fields, the idea of cross cutting collaboration starting from the equal leveling of PHAT and TT (as shown in Figure 6 above) is the next step. The starting point is market pull. Market pull/push demonstrates the inter-dependability of new technologies of the PHAT pipeline (in particular HNA and HTA) with TT activity. TT should work two-fold, using its traditional 'opportunity identifying' needs methodology fuelled by market demand and bilateral communication through evidence-based advocating for relevant (or upcoming) technologies in its activity with the PHAT organizations and DMs. This can be made possible through public-private partnerships (PPP). According to Laane et al. [45], academics, industry (in our case the academicindustrial complex) [23] and government join forces in PPPs to translate basic science into marketable applications with social and economic value. These PPPs can take different forms as it can be on a oneon-one basis or it can involve multiple parties (large to small medium enterprises) or universities to (semi) public or private research organizations and/or involve charities and government. Laane et al. [45] further states that PPPs can have physical locations or be virtual (with researchers from different organizations/locations) or it can be entirely different. PPPs are considered to be collaborative (partners work together contributing knowledge/resources), precompetitive (exclusivity not an issue in collaboration) and they are partnerships in which the risks/rewards, funding and intellectual properties are shared. PPPs are supposed to:

i. Bridge the gap between the academic-industrial complex and policy makers

ii. Pool knowledge and resources

iii. Catalyze innovation through translational research

iv. Transmit momentum and gear social/market pull to technology push

v. Multiple investments

vi. Align academic-industrial research agendas with social priorities

vii. Concentrate focus and mass on areas of social priority in which the parties are strong

viii. Increase social and economic returns of basic research 


\section{| CHAPTER |}

In our case PPPs also involve consultation. The industry should get involved in the subsequent steps of PHAT, including identifying the current and potential future needs, assessment, impact and prioritizing among others and should involve a feedback loop with communication two-way. This is a good strategy to ensure flexibility and adaptability by both parties leading to higher chances of success both commercially and policy-wise, which is principle of communicating vessels. For example, the health/market need and technology could be identified by the industry and possibly via consultation with the end users and DMs (say HNA). After initial research and development, the industry can approach the HTA professionals and DMs with their proposal to fulfill the need and collaborate through the HTA steps and vice versa. This also includes the assessment of the impact the technology will have on society (HIA) in mutual collaboration. In hindsight, this can help covering areas critically ignored by both, the industry and the health care system.

This combination brings into the picture the PHGW, which demonstrates the integration process of Genomics into Public Health and healthcare in general [42]. Beskow et al. [42] divides Public Health tasks within three key domains of Public Health (Genomics), viz., Assessment, Policy Development and Assurance. The industry and DMs should systematically and in parallel go through these domains at an early stage within our model and integrate it in their respective strategies of product development and development of evidence based guidelines. This is in addition to the TT and PHAT inter-dependability, to ensure areas to be covered or gaps are not neglected in their technology development and policy respectively. Thus all critical economic, policy and societal aspects are dealt with pre-emptively which are generally put aside. As a result, the timely as well as effective and efficient transition of relevant technological integration in policy and the health care system can be ensured.

As a consequence of these interactions and setups being developed, it is important that a sustainable infrastructure is in place which can accommodate these interactions since the major component of our strategy involves complex communication. Furthermore, the stakeholders involved should use the concept of this infrastructure to push forward their respective agendas within our 'LAL Model'. Keeping in mind this infrastructure, our model proposes to use/develop and gives rise to the (innovation) network which is an evolving mutual dependency system based on resource relationships in which their systemic character is the outcome of interactions, processes, procedures and institutionalization. Activities within such a network involve the creation, combination, exchange, transformation, absorption and exploitation of resources within a wide range of formal and informal relationships $[46,47]$. This 
network is dependent on the VOI (Value of Information). VOI is the maximum price one should pay for knowing the actual value of an uncertainty before deciding on a course of action [43] (see legend of Figure 6). In our case this can be a technology or technique in question to be integrated in health policy and healthcare. According to Oestreich [43] and adapted to our model, the VOI is dependent on three factors, namely relevance to the consumer, processing ability of the current infrastructure (the innovation network-developed through PPP between the academic-industrial complex and government, to understand say its clinical utility or utility in healthcare) and exclusivity to the provider (patent for example). This is a learning process with continued iterations and feedback loops, and requires the ability to learn, assimilate and use knowledge developed elsewhere through a process that involves substantial investments especially of an intangible nature and is called the absorption capacity $[47,48]$. 


\section{Discussion}

Based on our model, we believe the higher the absorption capacity, the higher the possibility of success as can be seen from Figure 7A below. The $y$-axis represents the progress and development of the innovation network. The numbers on this axis are percentage units of the expansion of the innovation network with 0.1 being $10 \%$ and 0.8 being $80 \%$. The x-axis represents time in years, which corresponds to the timeline of the TT activity. This graph in ideal situations corresponds with the development of upcoming or new technologies. With regard to relevant technologies already in the market, the same can apply, however the years may be shorter. The gradient represents the absorption capacity, which in our case is the function of the innovation network with respect to time which in turn in dependent on the VOI. The threshold or optimal possibility of the absorption capacity is marked at $80 \%$ in (say) 5 years. It can also be noticed that the absorption capacity gradient does not start at year 0 but rather a bit later. As earlier mentioned, the initial stages of TT start in the lab with research and development and the research is motivated on market dynamics and cannot clearly identify with the PHAT's Health Needs Assessment, which in turn is the necessary partner (HTA) of TT, required for the expansion of the innovation network. Therefore progression cannot start at this stage. After this step, within the TT pipeline, the innovation network becomes activated. Below is the proposed idea of the progression:

1. Identification of the health need (market pull/push) by industry and subsequent research and development. We mark this as $0 \%$ innovation network.

2. After initial research and development, contact is made with the relevant authorities in HNA/HTA and policy. We consider this $10 \%$ development of the innovation network, which starts from $0 \%$.

3. A proposal is developed either jointly (PHAT and industry) or individually (say academiaindustry) and it is advocated. Networking is the key here. We consider this $20 \%$ development of the innovation network.

4. Initiation of PPP of the industry with policy through the PHAT authorities. Partnership can be in terms of collaborations, knowledge sharing, joint investigations, etc. This is a major milestone; therefore we consider this $40 \%$ capacity of the innovation network. 
5. Over time the innovation network is developed and expanded as the industry collaborates with decision-makers through the process of the PHAT and TT activity pipelines which can look like a pseudo-merger. We consider this $60 \%$ network capacity.

6. Finally, the innovation network has matured enough to integrate relevant technologies into healthcare systems and policy in real time as a consequence and evaluate the impact using HIA. We believe the innovation network at $80 \%$ functionality and are considered the threshold of the absorption capacity of this apparatus.

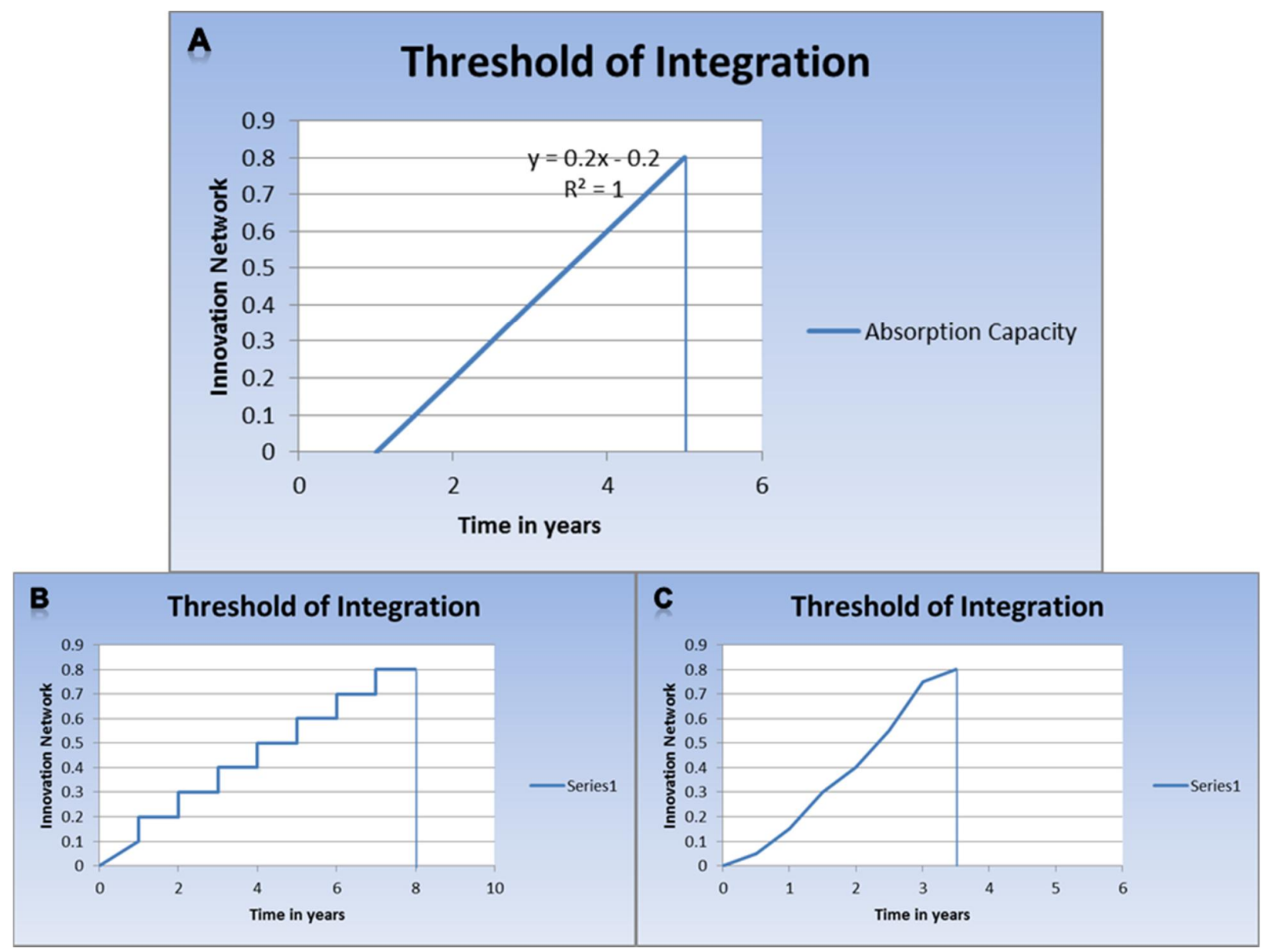

Figure 7: Absorption Capacity as a function of the innovation network and time. A hypothetical schematic representation from top counter-clockwise of achieving threshold capacity for technological integration in (A) ideal circumstances, (B) for a newly introduced technology in a non-existing innovation network with the network development hindered through bureaucracy and skepticism of the new infrastructure, (C) of an already tested innovation network moving through a 2 nd round of new technological introduction. 


\section{| CHAPTER |}

The steps take into consideration the PHGW. The value of 1.0 or $100 \%$ seems not feasible as this is a learning process and there will be always room for improvement apart from the fact that there will be always unforeseen internal and external developments. This example shows emerging technology integration via the development from a non-existent innovation network being expanded. The remaining $20 \%$ is an ongoing process with feedback loops, within the already now developed innovation network (above 6 steps) for more new or relevant technology integration; thus having a dynamic absorption capacity. This would be considered ideal in the development of the innovation network; however, depending on the technology to be integrated [49] the graph can and will be represented differently for different people/organizations over time as shown in Figure 7B and 7C below.

The involvement of various stakeholders is an essential in order for this model to work. Ideally, government driven regulatory requirements would greatly benefit the model by stimulating the adoption of the model for the right reasons. PPPs, for example, act as a cog in the larger innovation system conveying momentum from one part to the other and are best if the push is from technology and the pull is from social and market demand [45]. As of now the right level of intervention settings and involvement is not ideal. Currently, it is up to the industry to voluntarily advocate and/or implement the model in their settings to benefit. In the process, and as the model indicates, PPPs will bolster the involvement and perhaps future government driven regulatory requirements. Likewise, if decision makers identify the benefits of this model they can voluntarily indicate it to the industry to follow suit. Either way, in realistic setting, one core stakeholder has to take the initiative to kick-start the framework. The core stakeholders to initiate the model are basically the industry (including scientists in other settings) and government decision-makers including PHAT professionals. Other important stakeholders to sustain the process are the patient groups, hospitals and health professionals (including doctors).

It should be emphasized here that since this process involves advocating and PPP, the model's ultimate goal is to benefit the end-consumer, the patient, in a timely and effective manner, and in the process stopping the rightful profit backlog of relevant or upcoming technologies that generally do not reach the patient. As earlier stated, this is dependent on the relevance to the end-consumer combined with the processing ability of the information generated through PPP with exclusivity to the provider. For industry, profit is primary motivation behind innovation research in medicine or healthcare in general. This is not wrong as long as the health of the patient is not compromised through misguided facts of (ir)relevant technologies diffused into the health care apparatus thus affecting the policy-making as a 
whole. This may or may not arise from market push. In order to avoid this, the industry is encouraged to consider ethical behavior when embarking in this model. To keep this in check, HTA professionals and DMs should always have a third advisor. The first being industry, second being the HTA experts and their sources (neutral or evidence-based recommendations), and the third being a combination of neutral external experts, healthcare providers (e.g. doctors) and patient interest groups. The reason to keep it in this order of recommendations is to encourage the pseudo-merger of $\Pi$ and PHAT. The industry can advocate for relevant or upcoming technologies in its pipeline based on sound scientific data and health needs through consultation with doctors and patients, in the PHAT pipeline. As a result, the industry needs to positively influence the DMs and HTA professionals to push forward the correct agenda for timely interventions. This can be possible if the industry communicates with the PHAT professionals evidently, hence the first opinion. However, the PHAT infrastructure should not compromise its own recommendation. The third opinion(s) being the most important and the motivation of the target group is self-explanatory as it is the patient group and they aim for improving quality of life through improving health and reducing burden of disease.

As a result, the model promotes an ethical balance between profit and improving health through timely and effective interventions. It should be noted here, however, the classical PHAT apparatus or methodology needs to be compatible with the current trends, which is not the case at the moment. Although revised versions of HTA like 'core HTA' [50] have been established, nonetheless, they have not proven sufficient enough to solve current issues. Therefore, a more innovative approach [51] compatible with our model is required, which will be our next step of investigation. Also TT may need to adapt to HTA as well. However, this is within the concept of innovation networks.

A possible limitation of the model may or may not include its lack of addressing the phasing out of an obsolete technology. Depending upon the stakeholder this may be of importance. Phasing out a no longer required technology may not be of concern for an industrial player looking to capitalize on the market with their new technology and seems to be the general perception based on the authors' experience. A very simple example can be microarrays. To our knowledge we have never seen a microarray company phasing out its previous best seller over a newer version (although they phase out the production internally depending upon demand). Rather they simultaneously sell both until the older version fades away. Reason being at the end of the day it boils down the amount of sales. This phasing out of obsolete technologies rather falls on the ears on hospital management, policy makers among 


\section{| CHAPTER |}

others. For them phasing out is equally important as phasing in a new technology. On the other hand it can be argued that industry should pay to a certain extent attention on phasing out obsolete technology parallel to their diffusion of own technologies. This can probably ensure a smooth integration process in healthcare as it will develop a good case for integration of emerging technologies in the healthcare and policy. Our LAL model itself does not address this important issue rather focuses on the phasing in or diffusion of a new technology in healthcare and policy. Nonetheless, our hypotheses is that the same model framework could be used simultaneously while phasing in a new technology, to phase-out an obsolete version, but still has to be experimented, which is like-wise for the phasing in concept itself. 


\section{Conclusions}

The LAL model proposes to facilitate and/or speed the valorization process of new and relevant technologies within healthcare systems with less chances of failure through early on involvement of stakeholders (within TT and PHAT). The aforementioned early on involvement of stakeholders can help the industry to come to an advance decision whether or not to continue with the developing/emerging technology or consider upgrading it before introducing it in the market. This can be based on for example the technology's clinical utility (is a measure of the health care value provided by the technology) [52] among others (HTA, etc.) thus saving on resources. Our model has potential to guide valorization in context with integration in health policy and healthcare systems resulting in the timely as well as effective and efficient introduction of relevant genome-based technologies.

Valorization generally neglects the policy side, hence the generally fewer successes of investments by Venture Capitalists in business models targeting healthcare. This new model proposes to bring in fresh components to the valorization process of scientific breakthroughs not only to the bedside but also to the healthcare system as a whole; thus making the process more efficient in real-time, by solving the backlog of relevant technologies in healthcare before they become outdated due to introduction of upgraded or newer technologies. The added value of this model is that it brings together, as per our knowledge for the first time, two separate entities (TT and PHAT) to early on involvement. As a result this can benefit both health care policy and industrial profit. It encourages a large amount of networking and communication with relevant stakeholders in order to be successful, demonstrates the interdependability and parallel initiation of policy and technological innovation. This network builds on the ongoing absorption capacity of the apparatus through an ethical balance between profit and timely interventions for the benefit of the population health. In simplistic terms it is a process of 'Learning, Adapting and Leveling', which in essence is the LAL model. 
1. Business Insights: Trends in Biotechnology Technology Transfer to 2014-Deal volume has doubled since 2008. [http://www.reports-research.com/168/d/2011/02/14/business-insightstrends-in-biotechnology-technologytransfer-to-2014-deal-volume-has-doubled-sin/].

2. Byrd CA: Profile of Spin-off Firms in the Biotechnology Sector: Results from the Biotechnology use and Development Survey-1999. Business Special Surveys and Technology Statistics Division Working Papers Statistics Canada; 2002.

3. Strength and Opportunity The landscape of the medical technology, medical biotechnology and industrial biotechnology sectors in the UK. HM Government; 2009, 11-12, 28, 34, 38, 40.

4. Maggon K: Global Biotechnology Market Intelligence Monograph. Knol Publishing Guild;, 32 2011.

5. US Biotech Market Analysis. RNCOS Industry Research Solutions; 2010.

6. Bercowitz SF: Biotech made easy. [http://www.mnn.com/green-tech/researchinnovations/stories/biotech-made-easy].

7. Ukropcova D, Sturdik E: Biotechnology commercialization in the world. Acta Chimica Slovava 2011, 4:115-125.

8. Niosi J, Banik M: The evolution and performance of biotechnology regional systems of innovations. Cambridge Journal of Economics 2005, 29:343-357.

9. Fontanarosa PB, DeAngelis CD: Basic science and translational research in JAMA. JAMA 2002, 287:1728.

10. Berwick DM: Disseminating Innovations in Health Care. JAMA 2003, 289:1969-1970.

11. Peng W: Trends in the biotech literature 2009. Nature Biotechnology 2010, 28:887.

12. Snyder M, Cook-Deegan B: DNA Patent Database Statistics: 2010. DNA Patent Database; 2011.

13. Catalogue of Biotechnology Patents in the Mercosur. BIOTECHSUR; 2008.

14. Murtagh J, Foerster V: Managing Technology Diffusion-Discussion Paper. Canadian Agency for Drugs and Technologies in Health; 2009, 4, 7-8, 10.

15. Khoury MJ, Gwinn M, Yoon PW, Dowling N, Moore CA, Bradley L: The continuum of translation research in genomic medicine: how can we accelerate the appropriate integration of human genome discoveries into health care and disease prevention. Genetics in Medicine 2007, 9:665.

16. Copple RF, Wellons HB: Evolution of a Bio-Product. Corporate Counsel 2008, 65-66. 
17. Mullins B, Crowe J: Technology Transfer: A Roadmap. College and University Auditor 1999.

18. Outreach \& Tech Transfer. [http://www.ncat.edu/ divofres/ott/howto.php].

19. Rasmussen E: The Process of New Venture Creation in a University Setting. Accompanying measures \& survival of new firms: between Darwinism and assistance Montpellier; 2005.

20. Rosenköetter N, Vondeling H, Blancquaert I, Mekel OCL, Kristensen FB, Brand A: The Contribution of Health Technology Assessment, Health Needs Assessment, and Health Impact Assessment to the Assessment and Translation of Technologies in the Field of Public Health Genomics. Public Health Genomics 2011, 14:46-47.

21. HTA Definition. [http://www.eunethta.eu/Public/About_EUnetHTA/HTA/].

22. Health Impact Assessment: Main concepts and suggested approach Gottenburg consensus paper. Brussels: European Center for Health Policy; 1999, 4.

23. Crow MM, Tucker C: The American research university system as America's de facto technology policy. Science and Public Policy 2001, 28:2-9.

24. McGlynn EA, Asch SM, Adams J, Keesey J, Hicks J, DeCristofaro A, Kerr EA: The quality of health care delivered to adults in the United States. New England Journal of Medicine 2003, 348:26432644.

25. Woolf SH: The Meaning of Translational Research and Why It Matters. JAMA 2008, 299:211-213.

26. Williams A: Priority setting in public and private health care. A guide through the ideological jungle. Journal of Health Economics 1988, 7:173-183.

27. Ostrower F: Foundation Effectiveness Definitions and Challenges. The Urban Institute; 2004, 6.

28. Managing Clinical Effectiveness Definition.

[http://www.clinicalgovernance.scot.nhs.uk/section2/definition.asp].

29. Burke W, Khoury MJ, Stewart A, Zimmern RL: The path from genome-based research to population health: Development of an international public health genomics network. Genetics in Medicine 2006, 8:453.

30. Valorize. [http://www.merriam-webster.com/dictionary/valorize].

31. Wiedhaup K, Herben C, Meijer I: Diversity. In Partners in the polder A vision for the life sciences in the Netherlands and the role of public-private partnerships. Edited by: Laane C, Besteman K. Roland Berger Strategy Consultants; 2009:221.

32. Innovation Roadmap on Bio-based Feedstocks, Fuels and Industrial Products. Bioproducts Canada; 2003. 


\section{| CHAPTER |}

33. Traore N: Bioproducts development by Canadian biotechnology firms: findings from the 2001 Biotechnology use and development Survey. Statistics Canada; 2003.

34. Progress report\#1-Bioresources Final Research to Support Development of an Organization to Advance and Promote British Columbia's Bioproducts Industry. 2005.

35. Roozenburg N, Eekels J: The Basic Design Cycle. Product Design: Fundamentals and Methods 1995, 84-93.

36. The Fish Trap Model. In Delft Design Guide. Edited by: van Boeijen A, Daalhuizen J. Delft: Faculty of Industrial Design; 2001:.

37. Health Technology Assessment Process.

[http://www.cgh.uottawa.ca/WHOCC/projects/eo_toolkit/hta.htm].

38. The HTA process of DAHTA@DIMDI.

[http://web.archive.org/web/20090626065707/http://www.dimdi.de/static/en/hta/methoden/ prozess/index.htm].

39. Douw K, Vondeling H, Eskildsen D, Simpson S: Use of the Internet in Scanning the Horizon for New and Emerging Health Technologies: A Survey of Agencies Involved in Horizon Scanning. Journal of Medical Internet Research 2003, 5.

40. Brown IT, Smale A, Verma A, Momandwall S: Medical technology horizon scanning. Australasian Physical \& Engineering Sciences in Medicine 2005, 28:200-201.

41. Sarewitz D, Pielke RA Jr: The neglected heart of science policy: reconciling supply of and demand for science. Environmental Science \& Policy 2007, 5-14.

42. Beskow LM, Khoury MJ, Baker TG, Thrasher JF: The integration of Genomics into public health research, policy and practice in the United States. Community Genetics 2001, 4:4.

43. Oestreich T: The value of information Part 1-The Framework. Journal of Management Excellence: The Value of Information 2010, 8-9.

44. Martin MJC: Innovations as Technological Mutations. Managing innovation and entrepreneurship in technology-based firms John Wiley \& Sons Inc.; 1994, 43.

45. Laane C, Besteman K, Dongen Bv, Vugts J: Unity. In Partners in the polder A vision for the life sciences in the Netherlands and the role of public-private partnerships. Edited by: Laane $\mathrm{C}$, Besteman K. Roland Berger Strategy Consultants; 2009:, 18, 39, 40.

46. Tijssen RJW: Quantitative assessment of large heterogeneous R\&D Networks: The case of process engineering in the Netherlands. Research policy 1998, 26:792. 
47. Fischer MM, Fröhlich J, Gassler H, Varga A: Innovation, Knowledge Creation and Systems of Innovations. ERSA conference papers 2000, 5-7.

48. Cohen WM, Levinthal DA: Innovation and Learning: The two faces of R \& D. The Economic Journal 1989, 99:569-570.

49. Booth-Clibborn N, Packer C, Stevens A: Health technology diffusion rates. Statins, coronary stents, and MRI in England. International Journal of Technology Assessment in Health Care 2000, 16:781-786.

50. EUnetHTA WP4-Core HTA on Drug Eluting Stents. Pilot assessment European Network for Health Technology Assessment; 2008.

51. Becla L, Lunshof JE, Gurwitz D, Schulte in den Bäumen T, Westerhoff HV, Lange BM, Brand A: Health technology assessment in the era of personalized health care. International Journal of Technology Assessment in Health Care 2011, 27:118-126.

52. Clinical Utility of Personalised Medicine. National Health and Medical Research Council; 2011, 3.

\section{Acknowledgements}

This project is supported by a grant from the European Commission PHGEN II (Duration period: June, 2009-May 2012 EU-Project No. 20081302). This work is also supported by the Canadian Institutes for Health Research (CIHR), CIHR Institute of Genetics, the CIHR Institute of Health Services and Policy Research through a grant (no. ETG92250) to the APOGEE-Net/CanGèneTest Research and Knowledge Network on Genetic Services and Policy. We also like to extend our thanks to the research schools CAPHRI (program Public Health Genomics) and GROW of the Faculty of Health, Medicine and Life Sciences of Maastricht University for supporting this research. 

CHAPTER II

THE OVERARCHING FRAMEWORK OF TRANSLATION AND INTEGRATION INTO HEALTHCARE: A CASE FOR THE LAL MODEL

Published as:

Lal JA, Morré SA, Brand A. Personalized Medicine 2013, In Press. 


\section{Introduction}

Recently there has been substantial increase in relevant genome-based technologies to market. Compared to its utilization in healthcare systems, we notice a huge gap. In order to address this bottleneck we previously developed the Learning-Adapting-Leveling (LAL) model. In this article we aim to demonstrate the overarching reach of the model for translation to market and implementation into healthcare systems moving towards personalized healthcare.

\section{Methods}

We use qualitative logical reasoning with the LAL model as reference.

\section{Results}

We see that Technology Transfer, Health Needs Assessment, Health Technology Assessment and Health Impact Assessment are justified for their inclusion. In addition, the Public Health wheel is justified as a good reference frame along with value of information.

\section{Conclusions}

We conclude that as the LAL model covers all dimensions and tools for translation and implementation in a defined method, therefore can be considered as the overarching framework for translation and implementation in healthcare integration.

\section{Keywords}

Translational research, Personalized Healthcare, Health Needs Assessment, Health Technology Assessment, Health Impact Assessment, Learning-Adapting-Leveling, Public Health Genomics, Public Health Wheel, ACCE/EGAPP, Technology Transfer. 
The dynamic nature of biological systems at a molecular level is highly intricate. Computational power has greatly contributed to the partial elucidation of such systems and resulted in technological innovations like genome-based technologies (GBTs) [1, 2] for personalized healthcare application [3]. Genome-based constitutes all -omics deriving from the human genome and including its interacting with environmental factors. Technology can also include an intervention, technique, process, methodology and diagnostic kit/application as well as technology in the general sense [2]. In the past decade we have seen an exponential growth in these GBTs on the market [4-6] [101]. This is considered as a consequence of knowledge valorization and spin-offs [4, 7-9]. Valorization can be defined as 'transformation of knowledge into concrete new products, services and processes' [10]. Spin-offs can be defined as 'firms whose products or services develop out of technology-based ideas or scientific/technical know-how generated in a university setting by a member of the faculty, staff or student who founded (or cofounded with others) the firm' [11].

Knowledge valorization and spin-offs have become 'the' way in translation to the market, as there have been numerous studies [11-14] to interface the initially considered gap between the academic environment and the industry. This has to a substantial extent been achieved and is generally referred to as the academic-industrial complex [2]. However transitioning a technology from the lab to the market is not the only issue, as coverage and reimbursement among other issues need to be addressed in the whole translational research pipeline [102]. Consequently, integration and implementation seem as important terms when it comes to transfer of knowledge attributed to applied research and valorization of technologies beyond the market resulting in diffusion to healthcare systems. As generally known, this diffusion of GBTs takes quite some time to be readily taken up in daily practice unlike market penetration (5-10 years) [15]. The average of such diffusion depending upon the type of technology can be ranging on average from 10 years up to 20 years $[16,17]$. From this perspective it can be safely said that by the time the technology reaches widely accepted daily practice it can be considered comparatively irrelevant as a more effective and efficient technology becomes available on the market [2], the latter of which is not being implemented at that moment into healthcare and should be however, but will have to go again through the same process of the former. And by the time it does go through the process and is implemented, another better technology appears in the market. This plays 


\section{| CHAPTER ||}

out repetitively in our observation to be what we like to call the 'circle of destination'. Competition of technologies to survive in the market is indeed healthy for the economy, however if they do survive as the best, their implementation procedures are another issue, which is the circle of destination as they become irrelevant by the time they are implemented when compared to potential in the market.

\section{Learning-Adapting-Leveling model}

To this end, we had developed the LAL (Learning-Adapting-Leveling) model (see figure 1), which we previously published [2] of which a follow-up regarding the steps on how to use the model in a timeline has been recently accepted to personalized medicine [3]. This model identified the bottleneck for this circle of destination as the non-synergy between the academic-industrial complex and decision makers (policy) offering a solution framework. The model works on the pretext that the academic-industrial complex is developing a relevant technology, which could be beneficial to the healthcare system. It should be noted here that there are two types in academia, on the basic scientist producing knowledge and the other applied scientist resolving practical problems which can be done through development and commercialization of a product possibly resulting in a spin-off company in order to realize the solution's application. For the academic-industrial complex here, we denote the applied scientist from academia. However, sometimes the basic scientists' ouput can lead to the applied scientist's work. It is to be also noted here that although the academic-industrial complex defines the combination of academics and industry, it is also known that industry can develop (health related) technologies without involving academics at times. For the sake of simplicity, we refer to both these instances as synonymous with the academic-industrial complex; after all the people working in industry graduated in academics. This however true, can be an over-generalization. Nonetheless, this distinction is not necessary for the model as either combined or separated, they will eventually interact in the model and play the same role with the same aim for developing a relevant technology, which could be beneficial to the healthcare system, although motives may be diversifying. The business intentions of academics and industry when separated do not affect the functioning of the model, which is described subsequently. From henceforth, whether separated or together, the work of the academics together with industry or industry alone will be referred to as the academic-industrial complex. 


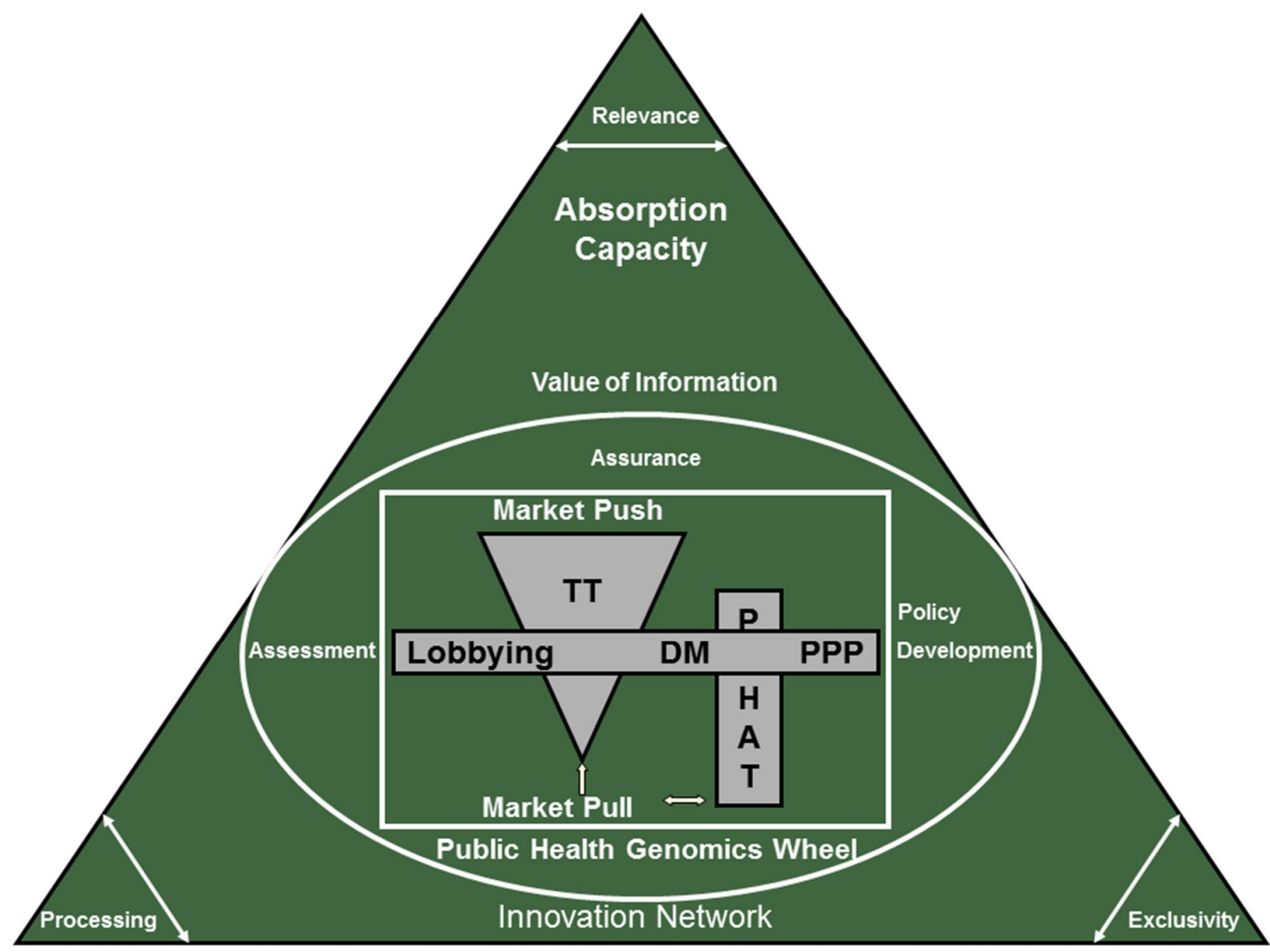

Figure 1: The LAL Model taken from [2] is based around the pseudo-parallel initiation of technology transfer (TT) and the public health assessment tools (PHAT). Pseudo-parallel is from the sense that the exact parallel can vary from case to case of the technology being considered. Also $\Pi T$ starting a bit earlier than PHAT indicates that $T T$ starts with basic research in the lab and at its preliminary stages, cannot be assessed with PHAT. TT is an inverse triangle indicating that basic/applied research starts in the lab with several feasible options and narrows down to a single concrete product to be developed. Both TT and PHAT are driven by the (market) need either by market push (developing a new need, mostly used by TT) or pull (addressing a need and penetrating that market). Market pull can lead to market push and vice-versa. These interactions are within a platform we call the innovation network and it is aspired that this possibly leads to developing broadly defined public-private partnerships (PPPs) through which TT lobbies for uptake of relevant technologies for healthcare through evidence-based reasoning. PPPs are eventually developed and improved upon which eventually reaches a critical mass we call the absorption capacity.

Coming back to the model (figure 1), at its core the model revolves around the pseudo-parallel initiation [3] and cross-talk of the Technology Transfer (TT) activity and the Public Health Assessment Tools (PHAT) [2]. TT in simplistic words is the development and transfer of the technology from the lab to the market for commercialization. It can be considered the de facto used by the academic-industrial complex, which we will come back on in the subsequent paragraphs. The PHAT [2] comprises of Health Needs Assessment (HNA), Health Technology Assessment (HTA) and Health Impact Assessment (HIA). PHAT is used to assess the current needs of the population, the technology and the impact the technology has on 


\section{| CHAPTER ||}

society respectively. Consequently, these determine the policy guidelines' development, reimbursement strategy, acceptance and implementation thus the integration of such technologies in healthcare $[2,3]$. Although these may be ideal but such evidence-based health policies are not descriptive of the actual innovation process. Decision makers (DM) use PHAT recommendations to develop these policies for healthcare. Due to this pseudo-parallel initiation of $\Pi$ and PHAT, the model encourages real time and early on involvement of all stakeholders (patient groups, insurance, industry, policy makers, investors, doctors, HTA professionals, academics, etc.) [2] as a result of these interactions. It can be noted here that through TT (which covers moving a technology from the lab to the market) and PHAT (which in a way moves the technology from the market to healthcare systems based on recommendations), the whole pipeline of non-synergy and translational research is aimed to be addressed.

In addition to its core and distinct advantage of early involvement of all stakeholders through the TT pipeline, the LAL model also constitutes and assesses the Value of Information (Vol). This Vol is divided into three parts (see figure 1) namely, evaluating the 'processing ability' of the end user (patient, doctor, etc.) with respect to understanding and using the technology, assessing the technology's 'relevance' to the target (population, patient, doctor, etc.), and understanding restrictions (exclusivity) due to intellectual property rights (IPR - limiting is wide usage), thereby developing solutions around these issues via consultation, cross-talk and public-private partnerships [2]. The model (figure 1) uses the 10 essential Public Health Tasks (within the domains of assessment, assurance and policy development) as developed first by the Institute of Medicine (IOM) in 1988 [18] and adopted later in 2001 to the field of Public Health Genomics [19]. This (known as the public health genomics wheel) is used as a reference frame to do control analysis of possible additional gaps (in parallel to PHAT) in technology conformity overlooked for public health integration [2].

So, in summary, the model assesses the feasibility of the developing technology from the start to near the end of the technology maturation process for real-time uptake by healthcare systems and policy guidelines. Thereby giving real-time recommendations to compensate for any gaps in the process prior to launching the product. As an output it builds and establishes partnership with policy and conforms the technology to be readily taken up by healthcare. This should not be considered as a business model rather a framework. The model applies differentially to settings when e.g. university hospitals directly implement their finding within their boundaries and related networks however are integrative when they venture outside their boundaries. Nonetheless, this is beyond the scope of the article. As earlier 
stated the model works on the pretext that the academic-industrial complex is developing a relevant technology, which could be beneficial to the healthcare system.

\section{Current status of the Learning-Adapting-Leveling model}

The LAL model has been integrated in the EU commissioned DG SANCO project PHGEN II (www.phgen.eu) for development of the first edition of 'European Best Practice Guidelines for Quality Assurance, Provision and Use of genome-based information and technologies' [20] and is being implemented in various European DG Research project ${ }^{1}$ and SMEs (Small Medium Enterprises) for genome-based technologies. Importantly, the LAL model has been implemented in the EU flagship pilot project ICT Future of Medicine (www.itfom.eu). Furthermore, apart from being referred to in literature [1, 3, 20-27] and conferences [28-31], mostly self-cited (refer to Google Scholar ${ }^{2}$ for further citations of reference 2) the model has been referred to in European high level reports [32-35]. As a result we like to consider the model an overarching framework, which defines how effectively and efficiently the real-time uptake of relevant technologies by the healthcare can be realized. This can help investors and industry alike to come to an early on decision on whether or not to further their technology, modify or end it, thus saving on resources. It is also important for patients in need for relevant GBTs possibly decreasing the burden of disease [3].

The article aims to build a case for the LAL model as an overarching framework as earlier stated. This can then help users (academics, industry, doctors, patient groups, clinicians, insurance, HTA professionals, etc.) to address all issues of translation and implementation to increase the likelihood of real-time integration of relevant personalized medicine related technologies (GBTs). The reason behind such an endeavor is that in the original article [2] we developed the conceptual model and detailed the development of its creation and the reasoning behind it. In our next article, we then elaborated on the detailed steps involved in the application of the model [3]. Given the model's expansion and usage as

\footnotetext{
${ }^{1}$ EU Framework Program 7 (FP7) --> COST - European Cooperation in the field of Scientific and Technical Research, Title: An integrated European Platform for Pancreas Cancer Research: From Basic Science to Clinical and Public Health Interventions for a rare disease; FP7-Health-2012-Innovation - Title: RARE Best Practices - Best Practice and knowledge sharing in the clinical management of rare-diseases. EU DG SANCO European Agency for Health Consumers --> Public Health Genomics European Network, Title: Best Practice Guidelines on Quality Assurance Provision and Use of Genome-based Information and Technologies. Canadian --> APOGEE Net CanGeneTest - A Research and Knowledge Network on Genetic Health Services and Policy, Pilot Project Title: Efficient integration of genome-based technologies into the healthcare system. Note: other grants in application.

${ }^{2}$ http://scholar.google.com/scholar?hl=en \&lr=\&safe=off\&c2coff=1\&q=link\%3Ahttp://www.translationalmedicine.com/content/9/1/207\&btnG=Search
} 


\section{| CHAPTER ||}

stated above in this paragraph, we now wish to elaborate why the model can be considered an overreaching concept for translation and integration into healthcare systems. Also these three topics are evident in their details, therefore had to be treated separately and each compliments and adds/builds on the other. Before we go into the methodology of the approach, we will first briefly describe generally accepted and used concepts in translation and implementation in this field.

\section{Translational Research and its coverage}

Commercialization or transfer of the technology from the lab to the market for us applies to the concept of TT whereas implementation applies to PHAT. Translational research covers the whole pipeline, i.e., TT and PHAT.

The term 'translational research' is generally considered from 'bench to bedside' [36], which encompasses the whole arena of translation from the lab (bench) to healthcare going beyond clinical applications (bedside).

\section{Encompassing properties of Technology Transfer}

For the academic-industrial complex, to our knowledge, transfer or commercialization of their concepts (in the lab) to technologies (on the market) is generally done through knowledge valorization. The complete concepts of knowledge valorization and spin-offs fall under the activity of $\Pi$ (see bottom part of figure 2). By definition, $T$ is as 'an activity of the migration of academic discoveries to useful application in the development of marketable products or processes' [37]. Such 'academic discoveries' are a product of 'translational research', which in turn is defined as the 'effective translation of new knowledge, mechanisms and techniques generated by advances in basic science research into new approaches for prevention, diagnosis and treatment of disease essential for improving health' [38]. It should be noted here that these are 'new approaches' and not the 'migration' itself of 'academic discoveries' to 'useful application' rather just 'new approaches'. TT migrates these 'new approaches' to 'useful application' (see figure 2). This 'migration' within TT is done through the concept of 'valorization', which is defined as already mentioned earlier in the introduction as the 'transformation of knowledge into concrete new products, services and processes' [10]. These products can be developed by regional valorization systems. 
Regional valorization systems can be defined as valorization systems from a 'regional perspective with an ecosystem of firms in the region, including customers, contractors, competitors and collaborators providing supporting services, potentials for subcontracting, knowledge collaborations and collective learning, aside from access to knowledge sources in the country and abroad' [39]. This can possibly be achieved through the concept of open innovations, which can be defined as 'any form of cooperation with third parties that can contribute to improve the long-term performance of a company' [40]. This may or may not result in spin-offs dependent on the origin of that knowledge; for example, establishment of an SME from the academic setting and/or through an established enterprise respectively. It can be said that spin-offs can be considered a possible product of knowledge valorization. However, these 'concrete new products, services and processes' need to be further developed to the form of 'marketable products or processes', which is within the definition of TT. TT therefore moves from basic research to the exit strategy of the product in question or to the market. Consequently, a spin-off is considered as one of several outcomes of TT.

TT is the most widely used and accepted activity [103] when it comes to the translation of technologies to the market and business plans and may have several variations from actor to actor. It should be noted here that as the definition of translational research contains the term 'effective translation' as well as 'bench to bedside', therefore it aims for the translation of evidence-based knowledge. In other words, it goes beyond the translation from lab (bench) to the market (using TT) and includes translation all the way to clinical practice and beyond, i.e. into healthcare (bedside), as can be seen in figure 2, the latter of which we will come back eventually in the text. The definitions above are subjective, can overlap and open to interpretation. However, we take the above concepts as they are mentioned when we talk about the encompassing properties of $\Pi$ for the academic-industrial complex to use for transferring concepts into technologies on the market. 


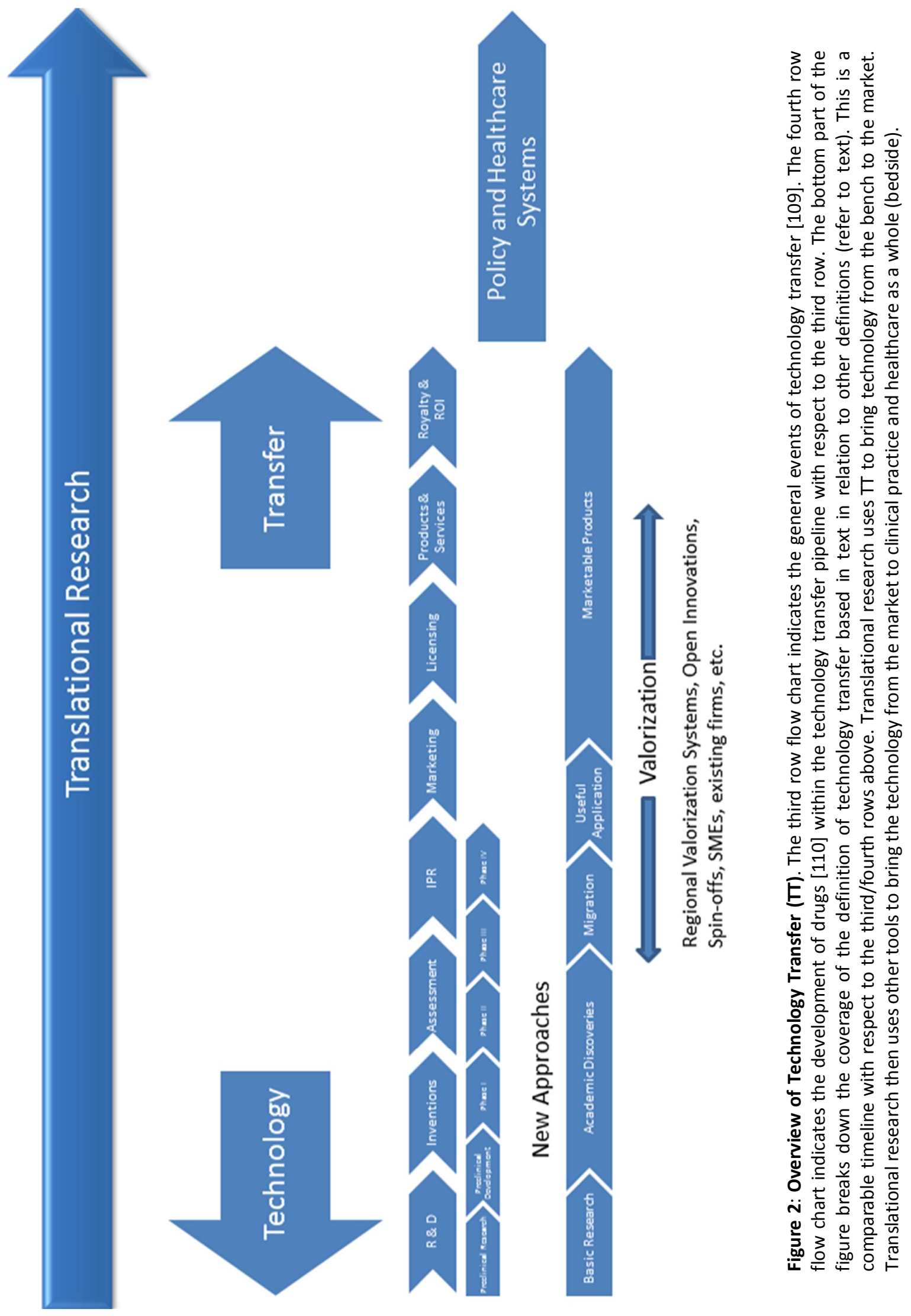




\section{Public Health Genomics}

After market introduction (through $\mathrm{TT}$ ) and before these technologies are taken up or implemented by healthcare systems and hospitals in daily practice they generally go through various assessments for their approval by decision makers generally unaware to basic scientists and industry (academic-industrial complex). There exist various assessment tools and frameworks in the Public Health Genomics field, which are considered important prior to uptake. Public Health Genomics is defined as 'the responsible and effective translation of genome-based knowledge and technologies into public policy and health services for the benefit of population health' [41]. Major tools used by decision makers directly or indirectly include Health Needs Assessment (HNA), Health Technology Assessment (HTA) and Health Impact Assessment (HIA) [2].

\section{Health Needs Assessment}

HNA is 'a systematic method of reviewing the health issues facing a population, leading to agreed priorities and resource allocation based on the needs of the population that will improve health and reduce inequalities' [42]. HNA can use epidemiological, qualitative and comparative methods to this end to address the above definition [43]. HNA works towards influencing policy through logical decision making, promoting interagency collaboration and advance prioritized R\&D [44]. The methodology of HNA involves first estimating the frequency of health issues in a given population and then evaluating the proof for the positive and negative effects of the technologies per health issue [45]. In the process, HNA addresses the impact, changeability, acceptability and resource feasibility with respect to maximum positive impact the technology can have on the health of a population, i.e. targeting whole populations, subpopulations as well as individuals [42].

\section{Health Technology Assessment}

HTA can be defined as a 'multidisciplinary process that summarizes information about the medical, social, economic, legal and ethical issues related to the use of a health technology in a systematic, transparent, unbiased, robust manner' [104]. HTA strives for the best possible value and also works on informing in the development of effective and safe (health) policies that are focused on the patient/citizen [104]. As this applies HTA's involvement in interventions and appraisals it is considered as an important information tool for decision-making [2]. According to Battista [46] HTA could be perceived to bridge the gap between decision-making and research, however, it is perceived to be based in 


\section{| CHAPTER ||}

scientific method [104]. Policy analysis, (health) economic evaluation and evidence-based medicine play a pivotal role in HTA. This is also extended to humanities/social sciences as well [47].

HTA is complimented by horizon scanning (HS - identifying emerging and new technologies) [48] and Constructive Technology Assessment (CTA). The latter addresses the issue of technologies being highly dynamic [49]. Historically HTA was developed from the need of healthcare systems and/or decision makers to identify economically relevant technologies for their usage [50,51]. However there does not exist a bridge between HTA professionals and the academic-industrial complex. Recently, an EU tender to address the issue was proposed [105]. On the other hand the American system has a chaotic documented history on HTA $[52,53]$.

\section{Health Impact Assessment}

HIA is 'a combination of procedures, methods and tools by which a policy, program, or project may be judged as to its potential effects on the health of a population, and the distribution of those effects within the population' [54]. In other words, HIA gives decision makers insights of the full spectrum of consequences of GBTs or policies as a result, as well as informs about unpredictability [55]. HIA, as a tool first investigates and determines various polices which can positively or negatively impact health. This is followed by considering both, direct as well as indirect health effects, thereby determining, which populations are affected as a result and on what basis. This is followed by reporting the findings to decision makers and finally evaluating the result of actual implementation [43]. HIA originated partly from environmental policy development, health equity and a social view of health [56].

In conclusion, HNA identifies 'health priorities for a given population', HTA evaluates the 'performance of health care technologies' and HIA assesses the 'effects of policies, programs or projects on the population's health' [43]. There are professionals and organizations, some designated by policy makers working on these separate 3 yet overlapping tools to develop recommendations for decision makers.

\section{The ACCE model}

On the other hand, the ACCE (Analytical Validity, Clinical Validity, Clinical Utility, Ethical, Legal, and Social Implications) is considered a benchmark framework to evaluate emerging genetic tests [57]. Supported and developed by $\mathrm{CDC}$, it aims to inform decision makers by presenting data on genetic tests in an updated readable format for them to make decisions [106]. From ACCE, Analytical Validity defines "how 
accurately and reliably the genetic test measures the genotype of interest' [106]. Clinical Validity defines 'how consistently and accurately the test detects or predicts the intermediate or final outcomes of interest' [106]. On the other hand, Clinical Utility defines 'how likely the test is to significantly improve patient outcomes' [106]. The last 'E' in the ACCE stands for ELSI (Ethical, Legal, and Social Implications) form an important part of the ACCE framework as implications may arise in context of using the genetic test [106].

The ACCE methodology includes gathering data, assessing it and interpreting the results followed by reporting that data to decision makers in an understandable updated read for them to come to a decision. This data is generally about DNA-related testing or genetic disorders [106]. A standard set of 44 specific questions comprises the model. These 44 questions are divided into 5 sections namely, disorder/setting (7 questions), analytical validity (10 questions), clinical validity (8 questions), clinical utility (16 questions) and ELSI (3 questions). Some of these 5 sections are also further divided into subsections [106]. The model has been both praised as well criticized (for it limitations) [57] for its different sections of ACCE. However, recommendations have also been given this is regard [57].

\section{The EGAPP Initiative}

EGAPP (Evaluation of Genomics Applications in Practice and Prevention) established in 2004 [107], can be in a way considered as the successor to the ACCE model as it builds upon ACCE and was also developed by CDC, USA. The EGAPP working group aims at the development of a 'systematic, evidencebased process for assessing genetics tests and other applications of genomic technology in transition from research to clinical and public health practice' [107]. The methodology constitutes existing recommendations based on epidemiological studies as well as knowledge from prior initiatives [107]. EGAPP consists of independent multidisciplinary panel of 15 experts sanctioned through CDC.

The general methodology involves selecting potential topics for review through horizon scanning and feedback, and then defining disorders of interest, specific tests and clinical scenario. This is followed by brief summaries of identified tests and a preliminary review and prioritization of topics using specific criteria ending in voting. The next step involves the EGAPP evidence review process. This starts with formulation of key questions and analytical framework for evidence review followed by a systematic evidence review either in the direction of comprehensive EPC (Enhanced Primary Care) reports or Rapid/Targeted reviews. After this, the EGAPP reviews the evidence reports, considers contextual issues 


\section{| CHAPTER ||}

and may consider other sources of evidence, and as a result a draft recommendation peer-reviewed statement is developed and published. This ends with the dissemination to stakeholders. Detailed step by step can be found in the document [58] CDC published.

\section{The T1 to T4 phases in translational research}

Khoury et al. [59] propose a framework for the continuum of multidisciplinary translation research. Although not part of the assessment used by decision makers, this framework has received attention [43]. The authors divide their framework into 4 phases. Phase 1 translation research (T1) aims to move a 'basic genomic discovery into a candidate health application'. This is followed by phase 2 (T2), which 'assesses the value of a genomic application for health practice leading to the development of evidencebased guidelines'. Phase 3 (T3) moves from 'evidence-based guidelines to health practice'. The final phase 4 (T4) evaluates in practice the health outcomes the genomic application. T1 to T4 can have feedback loops [59].

\section{The Public Health Genomics Enterprise}

The Public Health Genomics (PHG) Enterprise (see figure 3 below) is a composite 'for effective translation of genome-based knowledge and technologies into improved population health' [41]. The consensus was developed by an international expert workshop held in Bellagio, Italy in 2005 with 18 experts from US, Canada, Germany, UK and France. Although not part of the assessment process by policy makers, it nonetheless contains important components and overlaps with tools previously mentioned. The PHG Enterprise's knowledge integration is considered pivotal [41]. It can be defined as the process of selecting, storing, collating, analyzing, integrating and disseminating genome-based information both within and across disciplines for the benefit of population health'. This can also constitute methodological progression as well [41]. Further information can be seen from figure 3 below.

\section{The Public Health Wheel}

The Public Health Wheel adopted for Public Health Genomics (PHG) [19] can be seen from figure 4. This methodology has been widely used by the Public Health Genomics European Network (PHGEN I and II) [20] to develop European best practice guidelines for quality assurance, provision and use of genomebased information and technologies by different stakeholders at EU level. PHGEN II is a DG SANCO issued project with over 25 member institutions throughout Europe. The PHG Wheel demonstrates the 
integration of genome-based information and technologies into Public Health by addressing the 10 essential tasks of public health in the translation of the technology in question.

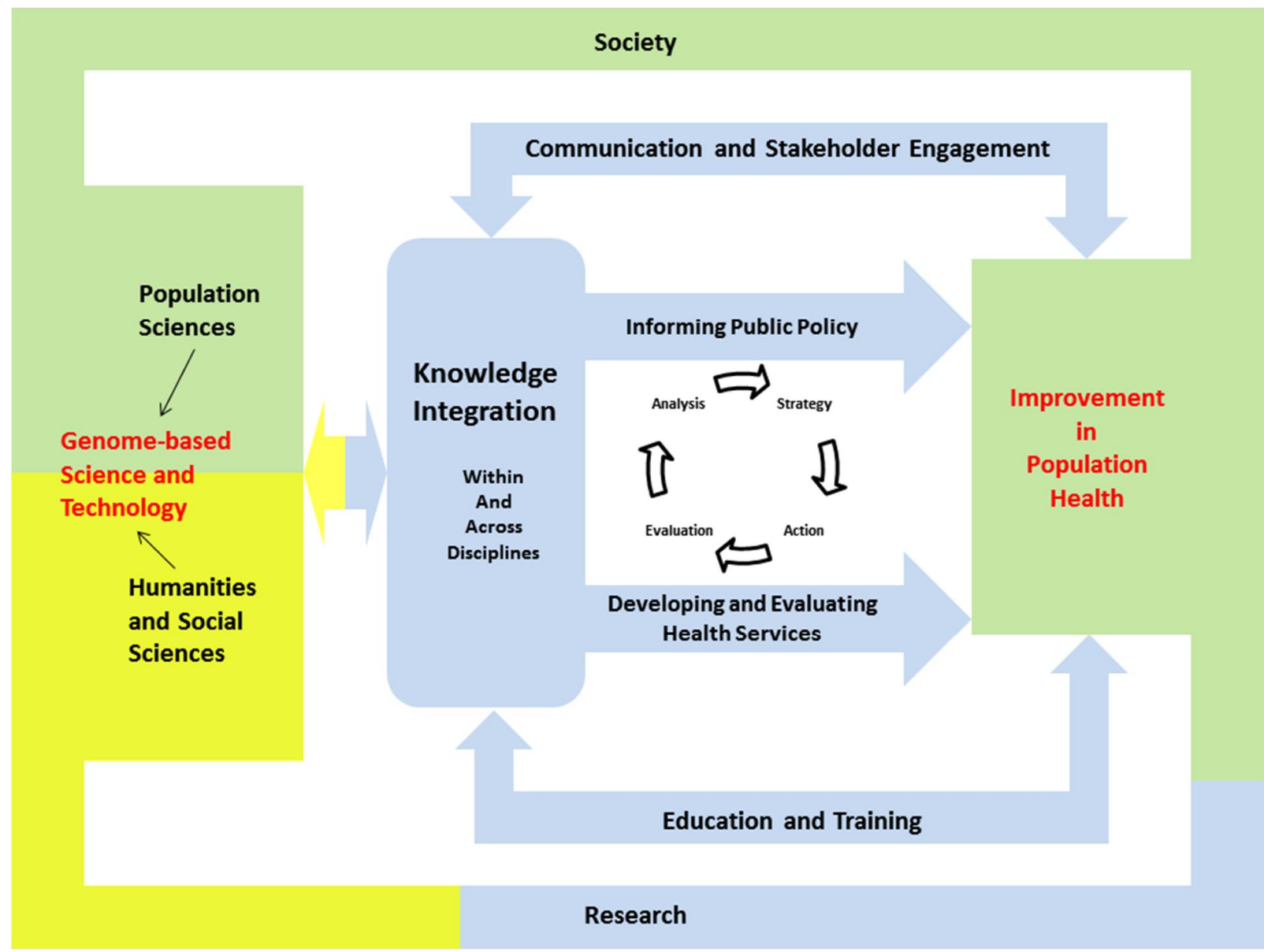

Figure 3: The PHG Enterprise - Blue represents the scope of the enterprise, which is the definition of PHG stated earlier in the manuscript. Yellow indicates creation of knowledge through research and red indicates genomebased science and technology playing a pivotal role. Green indicates society in its widest sense including stakeholders, activities, etc. Two sided arrows indicate dynamic and interactive nature of the enterprise. As can be seen the PHG enterprise has four main activities, viz. communication and stakeholder engagement, informing public policy, developing and evaluating preventive and clinical health services as well as education and training. Research identifies gaps with differentiation between basic and applied research being blurred. Also the generated knowledge is modulated by the results of its own output. This includes a cycle of analysis, strategy, action, evaluation, which is widely regarded in public health practice. Taken from [41] and adapted for resolution/color.

Further detailed definitions of these 10 essential tasks of Public Health within the 3 domains of assessment, policy development and assurance have been adopted and defined for the field of Public Health Genomics by Beskow et al. in tabular form [19], of which they can be found in table 1. 


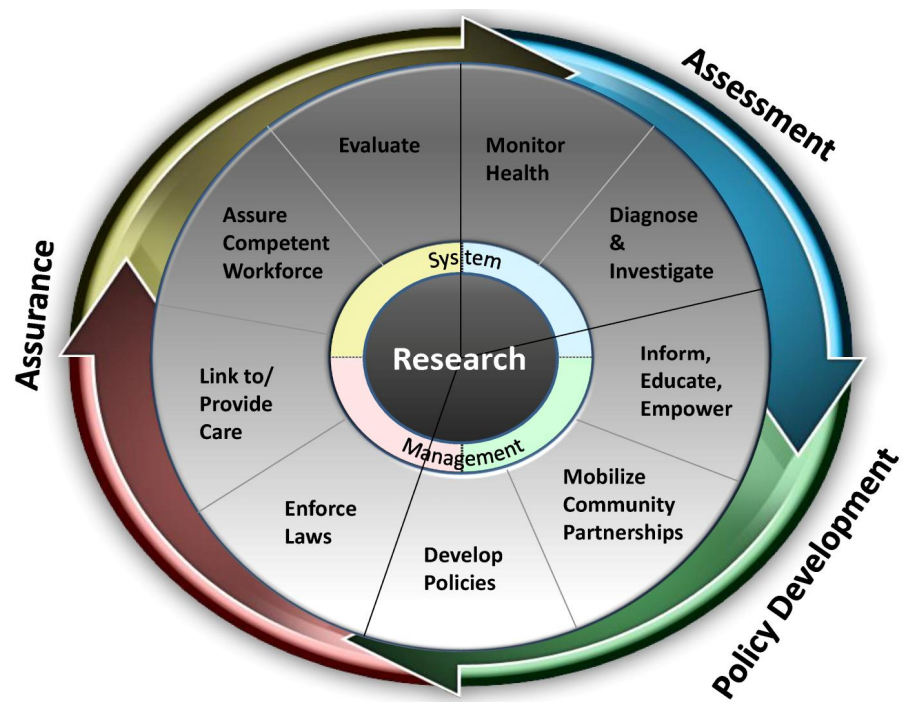

Figure 4: The PHG Wheel - The PHG Wheel demonstrating the 10 essential tasks of Public Health with the task research at its center. The 10 essential tasks can be distributed over the 3 domains of Public Health namely assessment, policy development and assurance with systems management at its center. Taken from [19] and adapted for resolution/color.

This summarizes the current and relevant tools, frameworks or models present for the market introduction and uptake of technologies in healthcare systems and hospitals with emphasis to the decision maker. We will now proceed to associate these different tools and frameworks through a common domain/framework and draw results and justification of an overarching existing framework. This can then help users to address all issues of translation before implementation to increase the likelihood of real-time uptake of relevant technologies in healthcare systems worldwide.

Table 1: Definitions of the task of the PHG Wheel. Taken directly from [19]

\begin{tabular}{|c|c|}
\hline Assessment & $\begin{array}{l}\text { Core function } \\
\text { The regular systematic collection, assembly, analysis and dissemination of information } \\
\text { including human genome epidemiologic information, on the health of the community } \\
\text { Related essential services } \\
\text { - Epidemiologic and laboratory research: quantifying the impact of gene variants on } \\
\text { human health and identifying and quantifying the impact on human health of } \\
\text { environmental risk factors that interact with gene variants. } \\
\text { Monitoring health: monitoring health status, including genetic factors, to identify } \\
\text { health problems within the community. } \\
\text { Diagnosing and investigating: investigating the distribution of genetic and } \\
\text { modifiable risk factors within the community to determine their contribution to } \\
\text { identified health problems and to improve health outcomes. }\end{array}$ \\
\hline Policy Development & $\begin{array}{l}\text { Core function } \\
\text { The formulation of standards and guidelines, in collaboration with stakeholders, which } \\
\text { promote the appropriate use of genetic information and the effectiveness, accessibility }\end{array}$ \\
\hline
\end{tabular}




\begin{tabular}{|c|c|}
\hline & $\begin{array}{l}\text { and quality of genetic tests and services. } \\
\text { Related essential services } \\
\text { - Policy and communications research: identifying and analyzing the economic, } \\
\text { social, ethical and political implications of advances in human genetics, including } \\
\text { the information and communications needs of stakeholders. } \\
\text { - Informing, educating, empowering: facilitating communications and education } \\
\text { about the integration of genomics into health promotion and disease prevention } \\
\text { programs. } \\
\text { Mobilizing partnerships: fostering collaboration between public and private } \\
\text { agencies and constituent groups to promote effective and efficient } \\
\text { communication and policy making about genomics. } \\
\text { Developing policies: establishing standards and guidelines for when and how } \\
\text { genetic information should be applied to promote health and prevent disease. }\end{array}$ \\
\hline Assurance & $\begin{array}{l}\text { Core function } \\
\text { Assuring constituents that genetic information is used appropriately and that genetic tests } \\
\text { and services meet agreed-upon goals for effectiveness, accessibility and quality. } \\
\text { Related essential services } \\
\text { - Health services research: identifying and analyzing the factors that influence the } \\
\text { impact of genetic information and the delivery, utilization and quality of genetic } \\
\text { tests and services. } \\
\text { Enforcing laws: promoting the enforcement of policies and standards enacted to } \\
\text { ensure the appropriate use of genetic information and the effectiveness, } \\
\text { accessibility and quality of genetic tests and services. } \\
\text { Linking to/providing care: ensuring the availability and accessibility of genetic } \\
\text { tests and services and associated interventions to improve health and prevent } \\
\text { disease. } \\
\text { Assuring a competent workforce: ensuring that present and future health } \\
\text { professionals have training and skills in the appropriate use of genetic information } \\
\text { to promote health and prevent disease. } \\
\text { accessibility and quality of genetic tests and services. }\end{array}$ \\
\hline $\begin{array}{l}\text { System } \\
\text { Management }\end{array}$ & $\begin{array}{l}\text { Building and maintaining the capacity of the public health infrastructure to integrate } \\
\text { genomics into public health research and practice. }\end{array}$ \\
\hline
\end{tabular}




\section{Methods}

The methodology used in the article to build a case for the overarching framework of the LAL model (figure 1) will be using the LAL model itself as a reference point [2]. The methodology will use a qualitative approach. Qualitative (logical) reasoning will deduct the priorities of inclusion or exclusion of various tools, frameworks/models or methods mentioned in the background section to the LAL model. Also, components included in the LAL model will be addressed to justify the model's overarching reach. The tools selected for description in the background and results section were based on our experience with working with such tools and our broader network of peers working in this field through our close work with academics, industry, governments as well as European networks or organizations like PHGEN II (www.phgen.eu), EUNetHTA (www.eunethta.eu), Euroscan (www.euroscan.org.uk), HTAi (www.htai.org), EAPM (www.euapm.eu), ESF (www.esf.org), EPIRARE (www.epirare.eu), PerMed among others and including information from CDC, USA. In addition, we also searched on PubMed for related terms. The PubMed search initiated with the terms based on our experience and networks above, and then expanded to terms based in those literature to include additional tools to ensure coverage of tools. As a result all tools are currently relevant and updated. The focus was to mostly include popular practical tools, which were previously used and exclude tools, which closely overlapped with selected ones or were adaptions. 


\section{Included Concepts (TT, HNA, HTA \& HIA)}

TT, given its place in literature and extensive usage [60-62] by the academic-industrial complex can be considered the most widely used and accepted activity [103] in translation from academic knowledge to products on the market. Being the benchmark in valorization and essential part of translational research (see background) in variety of forms, $\Pi$ tends to become an undisputed fact. Therefore it is logically a representation of translation of the academic-industrial complex and covers all aspects of translation. As a result it is considered one of the two pillars in the LAL model [2] speaking for the academic-industrial complex.

Same could be said for HNA, HTA and HIA, which we here refer to as PHAT [2], being the most widely used assessment tools [43] including its outreach through international organizations like the European Public Health Association's (EUPHA) 'Health Impact Assessment' section and HTAi and EUNetHTA among others. These tools (HNA, HTA and HIA) are mainly used at the local/national level (with some exceptions) for assessing technologies to be implemented in healthcare by decision makers (DM) and are well institutionalized [43], and therefore are the other pillar (PHAT) of the LAL model. As earlier stated the core of the model revolves around the pseudo-parallel initiation of TT and PHAT resulting in crosstalk, consultation and public-private partnership (PPP). The LAL model brings two important components, in fact in our view the most important components for translation (TT) and implementation (PHAT) respectively and encourages interactions between these two pillars.

\section{Excluded Concepts (ACCE, EGAPP, T1-T4, PHG Enterprise)}

The ACCE model and EGAPP Initiative

ACCE historically was developed from a human geneticists' perspective to evaluate specifically genetic tests as evident from use of the framework. It is based on a deterministic and linear understanding of genetics and genetic diseases. It is mostly limited to within the Unites States although there are indications of being used in other places [57]. On the other hand HTA is recognized worldwide ${ }^{1}$ and actively used for assessment of not only genetic tests, but in principle for all technologies in healthcare [108]. Furthermore, it is known that HTA also incorporates elements of ACCE when evaluating genome-

\footnotetext{
${ }^{1}$ www.htai.org and www.eunethta.eu
} 


\section{| CHAPTER ||}

based information and technologies [43]. Importantly, ACCE has been in a way considered to be expanded to EGAPP (as it builds on ACCE), although ACCE is still utilized. Apart from being criticized [57], ACCE is limited in its approach that it is from the perspective of human geneticists focusing on genetic testing assessment only, and only in the clinical setting as well.

The LAL model already having $\Pi T$ is from the perspective of the academic-industrial complex, which includes basic scientists including human geneticists, but also all omics' sciences, using translational research to develop new approaches to healthcare. A valid point in addition to this is that HTA, already included in the LAL model, is from the perspective of the requirements of the healthcare system and not what the human geneticists perceive as required. Aspects of ACCE have been covered in the LAL model under the concept of the reference frame of the Public Health Genomics (PHG) Wheel [19], which we will come back later in this text. Therefore, ACCE did not seem appropriate to include in the LAL model.

The same is the case for EGAPP, although incorporating parts of ACCE and encompassing the term genomic, is mostly limited within the boundaries of America however been documented elsewhere [63], is from the perspective of human geneticists and genetic epidemiology. Certain aspects are already covered as part of the reference frame of PHG. In summary, ACCE and EGAPP are limited in scope of geography, narrowed focus of the technology (genetic tests), and genetic epidemiology approach. Furthermore, it is already covered within the PHG wheel, parts of HTA already included in the LAL model. As a result ACCE and EGAPP were not considered at par with HTA therefore not included in the LAL model as such.

\section{The T1 to T4 phases in translational research}

T1-T4 phases as mentioned by Khoury et al. [59] like other similar models have been criticized by some [17]. It divides translation and implementation in 4 phases. In our previous article [2], in which the LAL model first appeared, we have defined our own phases. This includes translation from lab to industrial application as the first phase ( $\mathrm{T} 1$ for Khoury et al.). The transition from industrial application to market penetration being the second phase and the move from market to integration and implementation in policy is seen as the third phase [2]. Out of our own three phases, the first two can find home in TT of our model with the third allocated to the PHAT. Our last phase can be said as related to the T2, T3 and T4 of Khoury et al. is partly included in the LAL model, although from a different format. So the T1 of Khoury et al. falls within TT while T2, T3 and T4 fall under PHAT of our model. This also changes the timeline 
initially drawn by Khoury et al. as TT and PHAT run simultaneously. It is important to state that the idea of our own translational phases is not to add to the already existing models, which have been criticized for adding to the complexity like T1-T4 among others [17]. Rather taking T1-T4, which has been prevalent in literature (cited by 88 PubMed central articles and from $C D C$ ) and demonstrating it falls within our 3 phases.

The purpose of our own phases is to illustrate the steps of the LAL model. In other words, the term bench to bedside involves basic research possibly leading to applied research for an industrial application (our T1). The next step is to develop the technology for this industrial application and enter the market (our T2). Once in the market the aims should be to integrate or implement it in healthcare systems through best practice guidelines and health policy (our T3). We wanted to simplify multiple steps involved into 3 categories, which the LAL model addresses and is easier to focus with fewer phases involved.

\section{Public Health Genomics Enterprise and its relation to the Public Health Wheel}

Based on our own participation and discussions in the Bellagio meeting in 2005, and after reading both articles [19, 41], we consider the PHG Enterprise [41] and the PHG Wheel [19] as one and the same thing seen from a different perspective. Members of the Bellagio group [64] are authors on both papers with the PHG Wheel clearly defining all 10 essential Public Health tasks applicable to GBTs. This PHG Enterprise framework can be considered as rupturing up the pie chart of the PHG Wheel of figure 4 and converting it into a spread sheet. A lot of aspects overlap with the PHG Wheel, and as a result the PHG Enterprise covers all aspects within the assessment, policy development and assurance phase of the PHG Wheel (figure 4).

For the assessment phase (see table 1), just by looking at the PHG Enterprise aspects in figure 3, 'epidemiological' (population sciences, humanities and social sciences) and research data are covered on the research aspects of the enterprise (red color figure 3). 'Monitor health' spreads across the enterprise with the focal point being 'knowledge integration' (through developing and evaluating health services) towards improvement in 'population health' (see figure 3). Similarly, 'diagnosing and investigating' moves from research (keyword distribution) towards also in the improvement in 'population health' of the enterprise. Within 'policy development' all the tasks mentioned in the PHG Wheel (and table 1) can be clearly seen in the PHG Enterprise (see blue figure 3). In addition, 'health services research', 'linking 


\section{| CHAPTER ||}

to/providing care', 'assuring competent workforce' and 'evaluating' of the assurance phase of PHG wheel (figure 4 and table 1) are clearly seen within figure 3 (blue). These tasks of the PHG Wheel are however, in some cases merged or separated in the PHG Enterprise itself.

In addition, the 'System Management' of the PHG Wheel (figure 4, table 1) is covered in PHG Enterprise as a spread-out version of research (see the term 'research' in figure 3 ) with 'society' of figure 3 spread through the 10 tasks of the PHG Wheel (figure 4). However, the PHG Enterprise is not definitive in its steps rather a layout as such. On the other hand, the PHG Wheel is in a form of a pseudo-pie chart with the 10 essential public health tasks clearly defined, described and well distributed over Assessment, Policy Development and Assurance as can be seen from figure 4. Furthermore, the PHG Enterprise will be hard to use to as a reference frame as tasks are not defined rather distributed in terms sometimes vague. Therefore we decided to use the PHG Wheel instead of the PHG Enterprise for the LAL model's reference frame as the concrete tasks of PHG Wheel can easily be used to identify gaps besides the fact that these 10 essential tasks for our reference frame have been used to develop the best practice guidelines [20] at the EU level.

\section{PHG Wheel}

As stated above, given the clear and detailed tasks' distribution of the PHG Wheel, we have included it in the LAL model as a reference frame, which the LAL model consults through the pipeline of the parallel initiation of $\Pi T$ and PHAT. The concept of the reference frame is used as such that while going through HNA, HTA and HIA in the TT pipeline, common traits of HNA, HTA and HIA within the PHG Wheel are cross checked for coverage and expanded to other tasks of the PHG Wheel, thereby covering all aspects of public health.

Based on the definitions given in table 1 of the PHG Wheel (figure 4), and with reference to the definitions and aspects covered per tool or framework, we have assigned these tools or framework to sections of the PHG Wheel, which can be found in table 2 below. It should be noted that research is common to all tools/frameworks and corresponds to the research component of the PHG Wheel. Also the allocation of tasks per tool should not be considered absolute rather, as an indication. Please refer to table 2 throughout the text below. 
Table 2: Results of qualitative association of tools/frameworks to the PHG Wheel. Refer to table 1 and see text below for details. HNA is health needs assessment, HTA is health technology assessment, HIA is health impact assessment, ACCE is analytical validity, clinical validity, clinical utility and ELSI (ethical, legal and social implications), EGAPP is evaluation of genomic applications in practice and prevention, T1-T4 is translation phase 1 to translation phase 4 and PHG enterprise is the public health genomics enterprise.

\begin{tabular}{|c|c|}
\hline $\begin{array}{l}\text { Tools (see } \\
\text { background) }\end{array}$ & Common denominators to the PHG Wheel (see table 1 and figure 4) \\
\hline HNA & $\begin{array}{l}\text { Assessment: Epidemiological research, monitoring health, diagnosing and investigating } \\
\text { Policy Development: Policy and communications research, mobilizing partnerships, developing } \\
\text { policies } \\
\text { Assurance: Health services research, linking to/providing care, evaluating }\end{array}$ \\
\hline HTA & $\begin{array}{l}\text { Assessment: All aspects } \\
\text { Policy Development: Policy and communications research, mobilize partnerships, developing } \\
\text { polices } \\
\text { Assurance: All aspects }\end{array}$ \\
\hline $\mathrm{HIA}$ & $\begin{array}{l}\text { Assessment: Epidemiologic and laboratory research, diagnosing and investigating } \\
\text { Policy Development: Policy and communications research } \\
\text { Assurance: Health services research, evaluating }\end{array}$ \\
\hline ACCE & $\begin{array}{l}\text { Assessment: All aspects } \\
\text { Policy Development: Policy and communications research, informing, educating, empowering } \\
\text { Assurance: Health services research, evaluating }\end{array}$ \\
\hline EGAPP & $\begin{array}{l}\text { Assessment: All aspects } \\
\text { Policy Development: Policy and communications research } \\
\text { Assurance: None }\end{array}$ \\
\hline T1-T4 & $\begin{array}{l}\text { Assessment: All aspects } \\
\text { Policy Development: Policy and communications research, mobilizing partnerships, developing } \\
\text { policies } \\
\text { Assurance: Health services research, evaluating }\end{array}$ \\
\hline PHG Enterprise & $\begin{array}{l}\text { Assessment: All aspects } \\
\text { Policy Development: All aspects } \\
\text { Assurance: Health services research, linking to/providing care, evaluating }\end{array}$ \\
\hline
\end{tabular}

\section{Health Needs Assessment and the PHG wheel}

HNA in its aspects as stated (see background section), can use epidemiological data, and the definition of 'epidemiological data' within the PHG wheel in table 2 corresponds to identifying the health needs, therefore justifies its' presence in the same context within the PHG wheel. Similarly HNA covers 


\section{| CHAPTER ||}

'monitoring health' as the latter's definition key lies in it mentioning of identifying health problems within the healthcare systems. 'Diagnosing and investigating' also falls within the scope of HNA as it includes contribution (or impact) to identify health problems (needs) and relates to HNA's objective to improve health outcomes (PHG wheel). Within the policy development spectrum of the PHG wheel, HNA covers 'policy and communications research' as it relates to the identification of information and communication needs of stakeholders (can include the general population). 'Mobilizing partnerships' computes with HNA as both sections involve fostering (interagency) collaboration and promotion of policy through decision-making. 'Developing policy' is a key factor in the HNA process through both, allocation of priorities/resources and its support in decision-making.

In the assurance phase of the PHG wheel, HNA can be allocated to three tasks. 'Health services research', being the first, as it is a measure of the impact of genome-based information, quality, services, etc. can have, which can pave the way for the identification of the current health needs. The second task encompassing HNA is the 'linking to/providing care' aspect as the resource allocation and prioritizing of policy with aims to improve health of HNA is compatible to the definition. The last task being evaluating is quite obvious.

\section{Health Technology Assessment and the PHG Wheel}

HTA covers all tasks of the assessment sections (table 2) of the PHG wheel based on its definition. 'Epidemiological data' and basic research results are required for its assessment the latter of which form a rooted part of HTA. 'Monitoring health' and 'diagnosing/investigating' can fall under HTA's initial need assessment similar to HNA's above apart from covering aspects of the HTA's definition (safe, effective health polices, etc.). Also these two are required to complete the HTA analysis. Within policy development HTA can allocate to 'policy and communications research', 'mobilize partnerships' and 'developing polices'. For the first, the definition of HTA justifies its allocation as ELSI and economic aspects in the public health context are present. In 'mobilize partnerships'; policy-making plays a large role in HTA as well as consultation with external experts in order to get the best value (HTA). This is achieved through partnerships to sketch such policies. Developing polices through recommendations and formulation of health polices as per HTA form a key role in the assessment process. HTA is heavily dependent on policy analysis. Finally, HTA's role related to the tasks of the assurance phase of the PHG wheel, comprises the assessment of each stakeholder's contribution in fulfilling the tasks in complementary way ('who is doing what?'). 


\section{Health Impact Assessment and the PHG Wheel}

HIA can fall in all the three phases on the PHG wheel as well. Within assessment, it covers 'epidemiologic' and basic research as well as 'diagnosing and investigating'. The common denominator of these tasks with HIA is the keyword 'impact' (contribution in the latter). In other words, to study or measure the 'impact' of a technology epidemiological studies have to be done or are required. Similarly, one has to 'diagnose and investigate' to ascertain the possible impact of that technology. These are in context of societal, financial as well as organizational aspects. Within the 'policy and communication' task the definition aspects covered contribute to assessing the impact of a health technology or policy on healthcare. In the assurance phase, HIA can be associated with 'health services research' and 'evaluating' tasks of the PHG wheel. Again, the keyword for definitions on both sides is in analyzing the 'impact' of all kind of interventions.

Going through PHAT (HNA, HTA and HIA) as mentioned just above, in the TT pipeline for the LAL model [2], as can be seen, sections of the PHG wheel (table 1 and figure 4) are covered. Here we tally or crosscheck the PHAT to corresponding sections of the PHG wheel to fill in any gaps in the process addressing public health conformity of the technology required for PHAT. From here the expansion to unaddressed sections of the PHG Wheel including in non-traditional sense can be done to ensure all aspects of conformity are covered in the reference frame building a stronger case for decision-makers (including but not limited to reimbursement) and industry alike. We will now show that the left out components to the LAL model are still in a way covered through the PHG wheel known as the reference frame. This also justifies apart from previous reasoning, their exclusion.

\section{The ACCE model and the PHG Wheel}

The ACCE framework covers all aspects within the assessment phase (table 2) of the PHG wheel (table 1) from the perspective of the clinical setting and related to genetic tests. This is justified just by the abbreviation of ACCE compounding into the definitions, which make an obvious connection (see background section of ACCE). However, in the policy development phase, ACCE falls under the two tasks, namely 'policy and communications research' and 'informing, educating and empowering'. For the prior, ACCE by definition covers the ELSI aspects in the clinical context. Similarly, ACCE covers informing, educating and empowering' (see figure 5 below and definitions of ACCE) related to genetic counseling. 


\section{| CHAPTER ||}

For the assurance phase, ACCE associates with 'health services research' and 'evaluating' related to genetic services. In the case of the prior, quality assurance, effectiveness, economics and ELSI of genetic tests (see figure 5) within ACCE compute to the task's definition. The task of 'evaluating' genetic tests is as obvious as its name and definition, with some keywords from ACCE here being quality assurance, effectiveness, facilities, evaluation, etc.

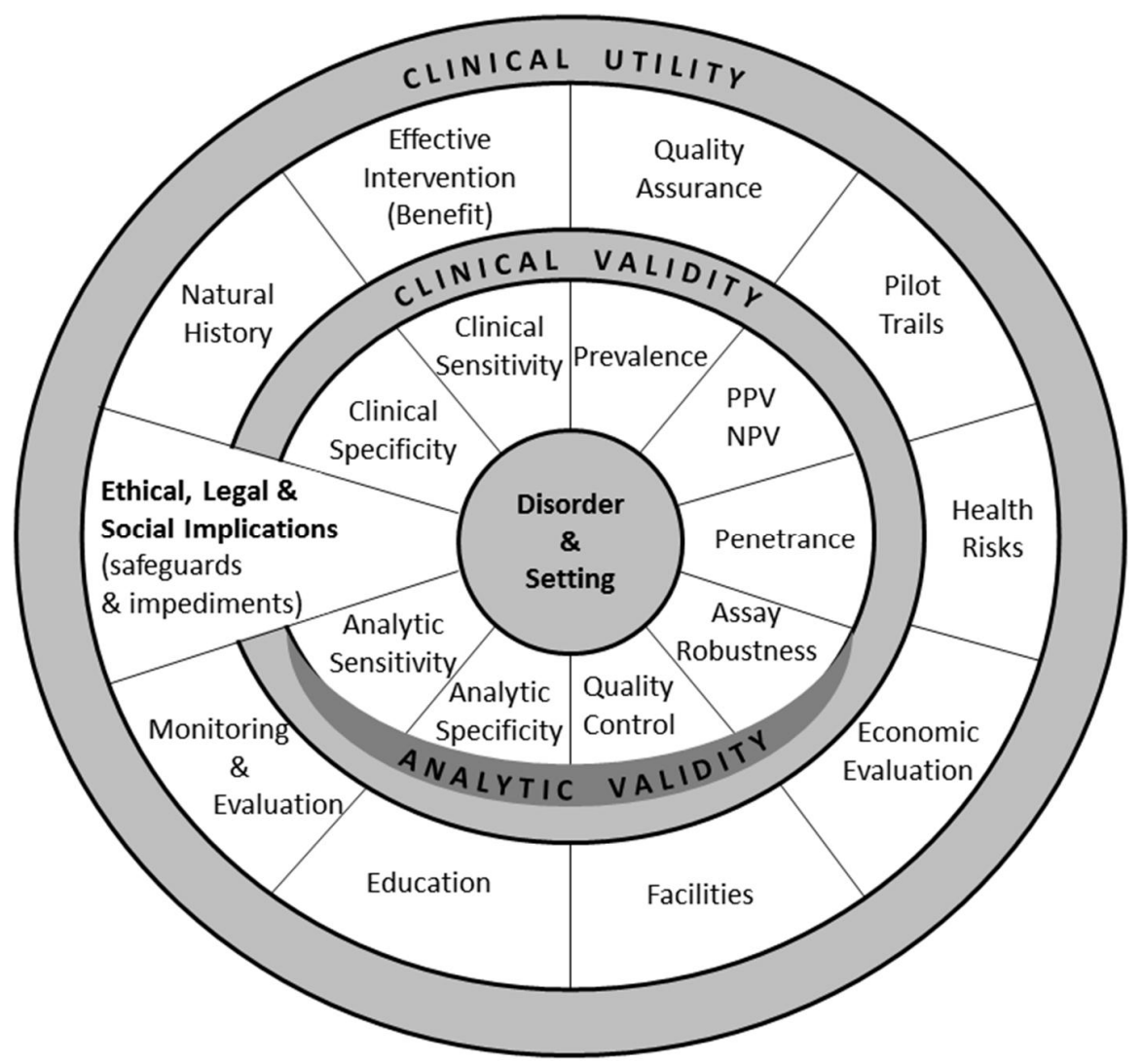

Figure 5: The ACCE - Sketch of the ACCE framework. Taken from [106]. Adapted for resolution.

\section{EGAPP Initiative and the PHG Wheel}

The EGAPP framework covers all aspects within the assessment phase of the PHG wheel (table 1 and 2) similar to the ACCE framework above and can be considered an upgrade of the ACCE with a more systematic approach, and from the perspective of (genetic) epidemiology. By definition it is an assessment tool for genetic tests, which implies its association within the assessment phase. The justification here can be similar in nature to the ACCE case for spectrum covered in the assessment phase including by just looking at the methodology of EGAPP. EGAPP covers the task 'policy and communication research' but does not have involvement in the assurance phase of the PHG wheel. In 
'policy and communications research', similar to ACCE, EGAPP covers ELSI aspects in the clinical context as well.

\section{T1 to T4 Translational phases and the PHG Wheel}

T1-T4 covers all aspects in the assessment phase (table 2) with T1 covering 'epidemiological data' as the starting point. T2 finds its way in 'monitoring health' as well as 'diagnosing and investigating' with the latter partly covered by T3 including T4. In policy development T1-T4 can be allocated 'policy and communications research' (T3-T4), 'mobilizing partnerships' (T1) and 'developing policies' (T2). In the assurance phase the framework can be placed with 'health services research' (T2) and 'evaluating' (T4). 


\section{Discussion}

As earlier stated the methodology approached the issue with a qualitative rather than a quantitative technique. Although this approach makes it more subjective and indicative, nonetheless, quantitative analysis cannot be practically measured within an association study for this aspect. We have justified well-known and applied tools to be included in the LAL model, which mainly constitutes TT and PHAT (HNA, HTA and HIA) and kept out others (ACCE, EGAPP, T1-T4 and PHG enterprise) due to their weaknesses and overlaps with existing tools (like PHAT and the PHG wheel). We have demonstrated that both included and excluded tools show relation with the PHG wheel as well. This demonstrates the importance of the PHG wheel as a reference in integration of genome-based information and technologies into public health and healthcare as a whole as it covers excluded tools which may have sections not covered otherwise.

\section{Another perspective on table 2}

Coming back to the subjectivity of the coverage parameters of these different tools in table 2 with respective to the PHG Wheel; it is an inclination towards a more indicative rather than an absolute description. Although our analysis was related more strictly to description of each tool then compared to the 10 essential tasks of the PHG wheel, it is nonetheless subjective. For example, a different view can be seen from table 3 below. Here some aspects covered are completely different from table 2 above unlike in table 2 some aspects of a domain were covered or not. Given the definition of HTA, and based on this table it seems that ACCE and EGAPP apparently do not perceive give way towards development of (health) policy. As a result these can be considered as non-HTA configurations. This is based on the usage of these different tools by different peers. This again is still subjective as other peers may see the coverage completely different. However, the important point stands that the PHG wheel has been demonstrated in the results section to be considered the most comprehensive of all these tools or the common denominator among these tools and serves as a reference frame for the tools incorporated in the LAL model.

\section{Placement of the PHG Wheel in the model}

The PHG wheel therefore extensively defines and covers all tasks in PHG. One can argue that why not then the PHG wheel is the second pillar along with TT in the LAL model instead of PHAT. The reason 
being that the PHG wheel only describes various public health tasks to be covered and not the methods how to do such tasks. On the other hand PHAT in the format of HNA, HTA and HIA are proven methods of assessment for healthcare interventions and their implementation thereby integration and widely used. Furthermore in a way HNA, HTA and HIA combined (PHAT) are methods that are applied to and cover the 10 essential task of the PHG wheel.

Table 3: Another perspective of the subjectivity coverage to the PHG Wheel. Comparable to table 2

\begin{tabular}{|c|c|}
\hline $\begin{array}{l}\text { Tools (see } \\
\text { background) }\end{array}$ & Common denominators to the PHG Wheel (see table 1 and figure 4) \\
\hline HNA & $\begin{array}{l}\text { Assessment: All aspects } \\
\text { Policy Development: All aspects } \\
\text { Assurance: All aspects }\end{array}$ \\
\hline HTA & $\begin{array}{l}\text { Assessment: All aspects } \\
\text { Policy Development: All aspects } \\
\text { Assurance: All aspects }\end{array}$ \\
\hline HIA & $\begin{array}{l}\text { Assessment: All aspects } \\
\text { Policy Development: All aspects } \\
\text { Assurance: All aspects }\end{array}$ \\
\hline ACCE & $\begin{array}{l}\text { Assessment: All aspects } \\
\text { Policy Development: None } \\
\text { Assurance: None }\end{array}$ \\
\hline EGAPP & $\begin{array}{l}\text { Assessment: All aspects } \\
\text { Policy Development: None } \\
\text { Assurance: None }\end{array}$ \\
\hline T1-T4 & $\begin{array}{l}\text { Assessment: All aspects } \\
\text { Policy Development: Policy and communications research, mobilizing partnerships, developing } \\
\text { policies } \\
\text { Assurance: None }\end{array}$ \\
\hline PHG Enterprise & $\begin{array}{l}\text { Assessment: All aspects } \\
\text { Policy Development: All aspects } \\
\text { Assurance: All aspects }\end{array}$ \\
\hline
\end{tabular}

\section{Relevance to Horizon Scanning and Constructive Technology Assessment}

There have been other initiatives like 'early HTA' [65] with the idea to promote pre-market evaluation for new upcoming technologies. Nonetheless, such initiatives lack the academic-industrial aspect $(\Pi$, see introduction section and figure 1). HTA now includes horizon scanning (HS) and Constructive Health 


\section{| CHAPTER ||}

Technology (CTA) as stated in the introduction section above. However the concept alone is more pseudo-real in nature. Reason being that there is no real-time communication between the academicindustrial complex and policy, therefore HS and CTA become limited to the knowledge they know or are provided with from the market. Recently, an EU tender call has been proposed to bring together HTA and technology developers (TT) to cross-talk [105] to answer aspects of this non-communication. Through our model by real-time cross-talk, true HS and CTA can be realized as policy is no longer limited to publicly known knowledge of upcoming technologies [3]. Through lobbying within the LAL model framework by $\Pi$, currently confidential or non-public emerging information can be tested for HS and CTA bringing into action the intended true nature of HS and CTA for assessing upcoming technologies within the HTA framework. As a consequence, HS and CTA hunting within HTA is made easy ensuring possibly missed technologies for assessment are covered as these innovators themselves approach HTA. Of course this does not replace the traditional HS and CTA approach rather supplements them and in the process defining their intended output.

\section{The overarching concept}

Furthermore, PHAT implementation process corresponds well with TT belonging to the academicindustrial complex, although not yet interactive. The LAL model addresses the issue of effective and efficient real-time integration of GBTs in healthcare by bringing together existing frameworks for the first time. Given the PHG wheel touching all assessment tools, makes it an excellent reference frame to go through all public health tasks to see if they are addressed or not in order for the final product to conform to public health needs as required by these assessment agencies (HNA, HTA and HIA).

Although the PHAT are extensive in approach and put together in the PHG wheel make quite a powerful approach in combination with the other tools, it not sufficient as the alienation to academics and industry makes this combination incomplete. The LAL model has this unique advantage bringing together TT and PHAT as well as the PHG wheel in the picture making it quite an extensive model. Moreover the LAL model takes into consideration the Vol in detail and develops the innovation network (see figure 1) through cross-talk, PPPs and consultation between TT and PHAT. The Vol runs in a same pattern as the PHG wheel (running in parallel to PHAT as a reference frame). Vol is important from two perspectives, namely from a business point of view and the traditional Vol of HTA [3] as approaches are different from these perspectives. This ensures that business aspects are covered and HTA's interest of the Vol is checked in advance before the HTA process. Based on these two aspects and Vol's well-known 
importance in decision making for both fields of work, namely business and healthcare decision making (HTA) it was decided to include Vol as the other important reference frame. The Vol through the steps of TT and PHAT always takes into consideration the processing ability of the users of the upcoming technology, relevance to the target group and restrictions of use due to Intellectual Property Rights (IPR). Hence, $\mathrm{Vol}$ recommends through the process to make sure the technology addresses these three areas. On how to use the Vol reference frame in parallel to the PHG wheel has been explained in our recent article [3].

It has been stated [66] that although several tools or concepts exist, their cross-integration is important in order to speed up the translation and currently no framework exists to do so. This article demonstrates the depth at which the LAL model can go when it comes to healthcare implementation as a result of the interactions between TT and PHAT with PHG wheel and Vol as the reference frames encompassing several tools resulting in cross integration. The PHG wheel in the LAL model guarantees a check list of aspects (tasks) and techniques or tools to be looked at through the pipeline of the innovation being developed making sure that all issues are covered apart from PHAT on a case by case basis. Also aspects not generally addressed in traditional PHAT (subjectively speaking like ACCE, EGAPP, etc.) can be addressed through the tasks of the PHG wheel. Thus they all go parallel in the LAL model (including ACCE, EGAPP which also overlap with HTA, etc. for example, but in the case of ACCE from the perspective of the evaluation of genetic tests in the clinical setting, and in the case of EGAPP from the perspective of genetic epidemiology). As result, the LAL model covers the whole wheel including gaps in the PHG wheel (in terms of TT), as well as concepts like the value of information among others, thus making it the best possible framework for healthcare integration.

\section{Pending issues}

It should be noted that TT in itself is very subjective and depending upon the actors' problems within TT can occur. However recommendations in literature has been given to this end [62]. This model is on the assumption that TT (academic-industrial complex) internally works out their issues possibly using our business Vol [3]. Nonetheless, TT is very active in transferring technology even if issues occur and the authors think it is not such problem to resolve any perceived differences and that interaction with PHAT is the bigger issue given the tools' independence from each other. 


\section{| CHAPTER ||}

Also with regard to PHAT, the protocols of the three components namely, HNA, HTA and HIA may vary from region to region and $\pi T$ will have to adapt accordingly, if they wish to conform the technology for that region and in some instances (like the US) the protocols may be carried out by a differently labeled agency [52]. Given these differences, a certain perspective and lobbying with organizations, networks, industry and decision makers including these three components (HNA, HTA and HIA) will be required to implement the LAL model. In this regard, we have already been active for some time now since the original article's publication in 2011 [2], which we briefly described in the background section stating where the LAL model has been published, integrated, lobbied and being currently implemented. Of course, organizational issues in some instances will have to be addressed and we still have a long way to go for it to be generally accepted. We have recently submitted an article defining the detailed steps involved to use the LAL model with respect to GBTs [3].

\section{Diffusion of Innovations}

We believe that the concept of 'diffusions of innovations' [67] is incorporated within the LAL model, although the idea of the model is not to study the concept rather to utilize and incorporate it through the LAL model's extensive interactions within the innovation network $[2,3]$ (see figure 1). Reason being that through these interactions in the innovation network of the LAL model between $\Pi T$ and PHAT as well as early on involvement of all stakeholders, the four main components that impact a concept according to Rogers [67] namely, innovation, communication channels, time and the social system are addressed. The 'innovation' is addressed through TT and its interactions with PHAT regarding its spread. 'Communication channels' are addressed and established through the interactions within the LAL model through the innovation network by the development of broadly defined public-private partnerships both formal and informal [2]. 'Time' in the form of the bottleneck of effective and efficient real-time integration is the basis from which the LAL model was developed. 'Social system' is incorporated through these interactions in the innovation network with TT conforming to the requirements of public health and health care systems via these assessments. Also the involvement of all stakeholders including patient groups, insurance companies, doctors, academicians, decision makers, HTA professionals, industry among others throughout the process of the LAL model ensures that the 'social system' the LAL model works within is addressed. Furthermore, the ethical, legal, social and economic implications are addressed through tools in PHAT. In addition human capital is an important aspect of the LAL model as it is according to Rogers. 
Also, the categories of adopters according to Rogers [67], namely innovators, early adopters, early majority, late majority and laggards are encompassed within the LAL model. The motive in the LAL model is to shift towards early adopters through the conformity requirements and stakeholder involvement during the implementation phase of an innovation early on. In addition, the concept of 'critical mass' in 'diffusions of innovations' can draw parallel with the 'absorption capacity' [2] of the LAL model (see figure 1). Using the concept of 'diffusion of innovations', the LAL model can develop the required interactions for effective and efficient real-time uptake of GBTs within its innovation network. In other words, even if the actors utilizing the LAL model are not familiar with 'diffusion of innovations' they are bound to use those concepts knowingly or unknowingly as a result of the complex interactions of stakeholders within the LAL model as concepts like social, change, mentality and organizational issues will be addressed in the LAL model. 
This article demonstrates the added value and comprehensibility of the LAL model as a result of inclusion of the PHG wheel in parallel and overlapping to the PHAT. We found that the PHG wheel to be a powerful instrument for reference of the different PHG tools in the form of the 10 essential public health tasks. However, we find that the PHG wheel is not sufficient for the integration process as it lacks the academic-industrial concepts like TT and Vol besides not having defined methods as well as not being directly involved with implementation unlike PHAT. The pseudo-parallel initiation of TT and PHAT are two sides of the coin of market introduction from lab to industrial product (TT) and implementation through PHAT (HNA, HTA, HIA), thereby integration. Using the PHG wheel as the reference frame in parallel to PHAT makes it a powerful combination and checklist to ensure the upcoming technology conforms to public health issues required by assessment agencies (PHAT). This reference frame includes possible overlooked sections and/or (of) excluded tools as well as limited perspectives (like ACCE, EGAPP, etc. due to previously mentioned reasons). The reference frame expands from PHAT to other sections of the PHG wheel. Similarly, Vol ensures the developing technology conforms to its accessibility in terms of its friendly platform, relevance to consumers and any IPR issues. The cross-talk critical for successful integration exists through the innovation network (figure 1) of the LAL model. Therefore the LAL model covers all aspects and components from the first idea in the lab to the product on the market (TT) to healthcare implementation (PHAT and PHG wheel) resulting in real time integration of personalized healthcare applications (GBTs) in healthcare systems. In conclusion, the LAL model should be used as the standard for healthcare integration as it covers extensively all important components for translation and implementation.

The LAL model identified 3 phases of translation [2] as earlier stated. As described in the article [2] the entities (academic-industrial complex and decision makers), which govern these phases through various tools (TT and PHAT respectively) have lack of communication, hence the bottleneck of real-time integration. The unique nature of the LAL model is that it brings together these two entities and their respective tools to cross-talk, PPPs and consultation, thereby developing the innovation network striving to an optimal (absorption) capacity to operate within (figure 1). The current article demonstrates, the LAL model incorporates relevant widely used tools for these entities, moreover, includes reference frames (PHG wheel in particular and Vol) to cover all possible gaps with feedback loops. Therefore, the 
LAL model should be the sought answer to the question of cross integration [66] of different concepts of public health and even beyond (through TT). In a nutshell the LAL model can be an overarching framework covering critical translation and implementation activities for personalized healthcare applications into the healthcare systems in a well described method [3].

\section{Future Perspective}

As given in the background section, the LAL model has been written into both accepted and ongoing grants and ongoing collaboration with SMEs. Also this model is being used in the EU flagship project ITFoM (www.itfom.eu) and other EU projects. The model has been lobbied for in the EU member states as well as integrated in the EU best practice guidelines for quality assurance, provision and use of genome-based information and technology for use by different stakeholders as well as high level reports. As a result of these best practice guidelines it has been presented at the EU parliament. Recently the EC has come up with a very specific tender, which completely fits to the model showing the direction the EU is interested. Furthermore, the model transcends various disciplines in its creation spanning from basic sciences, technology to translational research by technology transfer, public health and health sciences including HTA among others. We therefore see the sustainability of the model in the near future. Given all these activities we believe the model has a chance of being widely used in the next 5-10 years and can be eventually considered an overarching authoritative framework for translational research in personalized medicine and beyond to ensure GBTs reach in an effective and efficient manner the healthcare systems in real-time. However, in these next years the aim of the model would be towards demonstrating the results of the proof of concept of the model, which will feed into is spread.

\section{Executive Summary}

\section{Proposal of framework of translation into personalized healthcare}

- Through genome-based technologies we see a huge market development for personalized medicine.

- However, the time from market to healthcare integration is quite delayed.

- This results in disadvantage to both industry and healthcare.

- Previously we developed the Learning-Adapting-Leveling model to resolve this issue.

- We aim to demonstrate the overarching framework of this model in personalized healthcare. 


\section{| CHAPTER ||}

\section{Conceptual Demonstration}

- We see that technology transfer (TT), health technology assessment (HTA), health needs assessment (HNA) and health impact assessment (HIA) is quite useful for their inclusion in the model.

- Furthermore, the public health genomics wheel and value of information included in the model ensure that all aspects of public health tasks and business aspects are covered respectively.

- Additionally, the public health genomics wheel ensures the activities of all tools rejected in the model are addressed ensuring coverage.

Conclusion

- The LAL model can be considered as an overarching framework for translation from the lab to the market into the healthcare systems.

- In the next few years, the proof of concept will be tested. 


\section{References}

1. Lal JA, Sudbrak R, Lehrach H, Brand A: Functional Dynamics: From Biological Complexity to Translation and Impact in Healthcare Systems. Journal of Computer Science and Systems Biology 2013, 6:88-92.

2. Lal JA, Bäumen TSid, Morré SA, Brand A: Public health and valorization of genome-based technologies: a new model. Journal of Translational Medicine 2011, 9.

3. Lal JA, Vaidya A, Gutiérrez-Ibarluzea I, Dauben H-P, Brand A: The LAL Model: from theory to hypothesis of steps for implementation of basic genome-based evidences in personalized medicine. Personalized Medicine 2013, 10(7): 683-701.

4. Strength and Opportunity the landscape of the medical technology, medical biotechnology and industrial biotechnology sectors in the UK. In Book Strength and Opportunity the landscape of the medical technology, medical biotechnology and industrial biotechnology sectors in the UK (Editor ed.^eds.). pp. 11-12, 28, 34, 38, 40. City: HM Government; 2009:11-12, 28, 34, 38, 40.

5. US Biotech Market Analysis. 2010.

6. Maggon K: Global Biotechnology Market Intelligence Monograph. In Book Global Biotechnology Market Intelligence Monograph (Editor ed.^eds.), 32 edition. City: Knol Publishing Guild; 2011.

7. Ukropcova D, Sturdik E: Biotechnology commercialization in the world. Acta Chimica Slovava 2011, 4:115-125.

8. Niosi J, Banik M: The evolution and performance of biotechnology regional systems of innovations. Cambridge Journal of Economics 2005, 29:343-357.

9. Byrd CA: Profile of Spin-off Firms in the Biotechnology Sector: Results from the Biotechnology use and Development Survey-1999. In Book Profile of Spin-off Firms in the Biotechnology Sector: Results from the Biotechnology use and Development Survey-1999 (Editor ed.^eds.). City; 2002.

10. Wiedhaup K, Herben C, Meijer I: Valorization. In Partners in the polder A vision for the life sciences in the Netherlands and the role of public-private partnership. Edited by Laane C, Besteman K; 2009: 221

11. Rappert B, Webster A, Charles D: Making sense of diversity and reluctance: academic-industrial relations and intellectual property. Research Policy 1999, 28:873-889.

12. Lam A: Knowledge Networks and Careers: Academic Scientists in Industry-University Links*. Journal of Management Studies 2007, 44:993-1014. 


\section{| CHAPTER ||}

13. Debackere K, Veugelers R: The role of academic technology transfer organizations in improving industry science links. Research Policy 2005, 34.

14. Dooley L, Kirk D: University-industry collaboration Grafting the entrepreneurial paradigm onto academic structures. European Journal of Innovation Management 2007, 10:316-331.

15. Stewart JJ, Allison PN, Johnson RS: Putting a price on biotechnology. Nature biotechnology 2001, 19:813-817.

16. Berwick DM: Dissseminating Innovations in Health Care. Journal of the Amercian Medical Association 2003, 289:1969-1970.

17. Trochim W, Kane C, Graham M, Pincus HA: Evaluating Translational Research: A Process Marker Model. Clinical Translational Science 2011, 4:152-162.

18. The Future of Public Health. In Book The Future of Public Health (Editor ed.^eds.). City: Institute of Medicine 1988.

19. Beskow LM, Khoury MJ, Baker TG, Thrasher JF: The integration of Genomics into public health research, policy and practice in the United States. Community Genetics 2001, 4:4.

20. Brand A, Lal JA: European Best Practice Guidelines for Quality Assurance, Provision and Use of Genome-based Information and Technologies: the 2012 Declaration of Rome. Drug Metabolism and Drug Interactions 2012, 27:177-182.

21. Brand A: Personalisierte Medizin: Von der Vision zur Realität. Bulletin SAMW 2012, 3112:2-4.

22. Brand A: Public Health Genomics and Personalized Healthcare: a pipeline from cell to society. Drug Metabolism and Drug Interactions 2012, 27:121-123.

23. Brand A: Public Health Genomics - from cell to society. The Newsletter of the British Society for Human Genetics 2013, 48:51-52.

24. Brand A, Morré SA: Public Health Genomics - public health goes personalized. Tijdschrift voor Gezondheidswetenschappen 2013, 91:86-87.

25. Lal JA, Malogajski J, Verweij SP, Boer Pd, Ambrosino E, Brand A, Ouburg S, Morré SA: Chlamydia trachomatis Infections and Subfertility: Opportunities to Tranlsate Host Pathogen Genomic Data into Public Health. Public Health Genomics 2013, 16:50-61.

26. Lal JA: Addressing the backlog of genome-based technologies in healthcare. The Newsletter of the British Society for Human Genetics 2013, 48:59-60.

27. Steuten L, Wetering Gvd, Groothuis-Oudshoorn K, Retèl V: A Systematic and Critical Review of the Evolving Methods and Applications of Value of Information in Academia and Practice. PharmacoEconomics 2013, 31:25-48. 
28. Lal J: Valorization of genome-based technologies in public health. In 4th European Public Health Conference; 10 November; Copenhagen. European Journal of Public Health; 2011

29. Vondeling H, Lal J, Douw K, Cassiman JJ, Brand A: Public Health Genomics and Health Technology Assessment. In 9th HTAi Annual Meeting; 24 June; Bilbao Spain. Gac Sanit.; 2012: 6.

30. Lal JA: Valorization of Genomics in Public Health. In Biomedica Life Science Summit; April; Eindhoven Netherlands. 2011

31. Lal JA, Bäumen TSid, Morré SA, Brand A: Valorizing Genomics in Public Health. In Genetica Retraite; February; Kerkrade Netherlands. 2011

32. Personalized Medicine for the European citizen. Towards more precise medicine for the diagnosis, treatment and prevention of disease (iPM). European Science Foundation Forward Look 2012:17,41.

33. ICT Future of Medicine Flagship Final Report. 2012:25,26,69,70,76.

34. Innovation and Patient access to personalized medicine. Report from Irish Presidency Conference March 20th/21st 2013. European Alliance for Personalized Medicine 2013.

35. Das war die Amgen Science Lounge 2013. AMGEN 2013.

36. Woolf SH: The meaning of Translational Research and Why it Matters. The Journal of the American Medical Association 2008, 299:211.

37. Mullins B, Crowe J: Technology Transfer: A Roadmap. In Book Technology Transfer: A Roadmap February edition. City: Association of College and Univeristy Auditors; 1999.

38. Fontanarosa PB, DeAngelis CD: Basic science and translational research in JAMA. Journal of the Amercian Medical Association 2002, 287:1728.

39. Geenhuizen Mv: Valorisation of knowledge: preliminary results on valorization paths and obstacles in bringing university knowledge to market. In The Eighteenth Annual High Technology Small Firms Conference; May 27-28; University of Twente, Enschede, the Netherlands. 2010

40. Wit Jd, Dankbaar B, Vissers G: Open Innovation: the New Way of Knowledge Transfer? Journal of Business Chemistry 2007, 4:11-17.

41. Burke W, Khoury MJ, Stewart A, Zimmern RL: The path from genome based research to population health: Development of an international public health genomics network. Genetics in Medicine 2006, 8:453.

42. Health Needs Assessment - A Practical Guide. In Book Health Needs Assessment - A Practical Guide. Health Development Agency; 2005. 


\section{| CHAPTER ||}

43. Rosenköetter N, Vondeling H, Blancquaert I, Mekel OCL, Kristensen FB, Brand A: The Contribution of Health Technology Assessment, Health Needs Assessment, and Health Impact Assessment to the Assessment and Translation of Technologies in the Field of Public Health Genomics. Public Health Genomics 2011, 14:46-47.

44. Wright J, Williams R, Wilkinson JR: Development and importance of health needs assessment. British Medical Journal 1998, 316:1310-1313.

45. Gray JAM: Evidence-Based Healthcare. How to Make Health Policy and Management Decisions. New York: Churchill Livingestone; 1996.

46. Battista RN: Towards a paradigm for technology assessment. In The Scientific Basis of Health Services. Edited by Peckham M, Smith R. London: BMJ Publishing Group; 1996: 11-18

47. Garrido MV, Kristensen FB, Nielsen CP, Busse R: Health Technology Assessment and Health Policy Making Current status, challenges and potential. In Book Health Technology Assessment and Health Policy Making Current status, challenges and potential (Editor ed.^eds.). City; 2008.

48. Robert G, Stevens A, Gabbay J: 'Early warning systems' for identifying new healthcare technologies. Health Technology Assessment 1999, 3:100-108.

49. Douma KF, Karsenberg K, Hummel MJ, Bueno-de-Mesquita JM, Harten WHv: Methodology of constructive technology assessment in health care. International Journal of Technology Assessment in Health Care 2007, 23:162-168.

50. Battista RN, Hodge MJ: The "natural history" of health technology assessment. International Journal of Technology Assessment in Health Care 2009, 25:281-284.

51. Stevens A, Milne R, Burls A: Health technology assessment: history and demand. Journal of Public Health Medicine 2003, 25:98-100.

52. Eisenberg JM, Zarin D: Health Technology Assessment in the United States. International Journal of Technology Assessment in Health Care 2002, 18:192-198.

53. Sullivan SD, Watkins J, Sweet B, Ramsey SD: Health Technology Assessment in Health-Care Decision in the United States. Value in Health 2009, 12:39-43.

54. Gothenburg Consensus Paper. Health Impact Assessment. Main concepts and suggested approach. In Book Gothenburg Consensus Paper. Health Impact Assessment. Main concepts and suggested approach (Editor ed.^eds.). City: HIA Gateway, West Midlands Public health Observatory; 1999.

55. Parry J, Stevens A: Prospective health impact assessment: pitfalls, problems, and possible ways forward. British Medical Journal 2001, 323:1177-1182. 
56. Harris-Roxas B, Harris E: Differing forms, differing purposes: A typology of health impact assessment. Environmental Impact Assessment Review 2011, 31:396-401.

57. Sanderson S, Zimmern R, Kroese M, Higgins J, Patch C, Emery J: How can the evaluation of genetic tests be enhanced? Lessons learned from the ACCE framework and evaluating tests in the United Kingdom. Genetics in Medicine 2005, 7:495.

58. EGAPP: Understanding EGAPP: Four Steps of the EGAPP Working Group Review Process. In Book Understanding EGAPP: Four Steps of the EGAPP Working Group Review Process (Editor ed.^eds.). pp. 2-7. City; 2008:2-7.

59. Khoury MJ, Gwinn M, Yoon PW, Dowling N, Moore CA, Bradley L: The continuum of translation research in genomic medicine: how can we accelerate the appropriate integration of human genome discoveries into health care and disease prevention. Genetics in Medicine 2007, 9:665.

60. Bozeman B: Technology transfer and public policy: a review of research and theory. Research Policy 2000, 29:627-650.

61. Heher AD: Benchmarking of Technology Transfer Offices and What It Means for Developing Countries. In Intellectual Property Management in Health and Agricultural Innovation: A Handbook of Best Practices. 2 edition. Edited by Krattiger A, Mahoney RT, Nelson L, Thomson JA, Bennett AB, Satyanarayana K, Graff GD, Fernandez C, Kowalski SP: MIHR Oxford, PIPRA Davis, Oswaldo Cruz Foundation Brazil, bioDevelopments-International Institute Ithaca; 2007: 207

62. Siegal DS, Waldman DA, Atwater LE, Link AN: Commercial knowledge transfers from universities to firms: improving the effectiveness of university-industry collaboration. Journal of High Technology Management Research 2003, 14:111-130.

63. Azim HA, Michiels S, Zagouri F, Delalogue S, Filipits M, Namer M, Neven P, Symmans WF, Thompson A, André F, et al: Utility of prognostic genomic tests in breast cancer practice: The IMPAKT 2012 Working Group Consensus Statement. Annals of Oncology 2013, 24:647-654.

64. Genome-based Research and Population Health. Report of an expert workshop held at the Rockefeller Foundation Study and Conference Center. In Book Genome-based Research and Population Health. Report of an expert workshop held at the Rockefeller Foundation Study and Conference Center (Editor ed.^eds.). City; 2005.

65. Ijzerman MJ, Steuten LM: Early assessment of medical technologies to inform product development and market access: a review of methods and applications. Applied Health Economics and Health Policy 2011, 9:331-345. 


\section{| CHAPTER ||}

66. Syurina EV, Bäumen TSid, Brand A, Ambrosino E, Feron FJM: Concepts for the translation of genome-based innovations into public health: a comprehensive overview. Personalized Medicine 2013, 10:163-176.

67. Rogers EM: Diffusion of Innovations. 5 edn: Free Press; 2003.

\section{Internet References}

[101] http://www.mnn.com/green-tech/research-innovations/stories/biotech-made-easy

[102] http://www.genome.gov/19016729

[103] http://www.reports-research.com/168/d/2011/02/14/business-insights-trends-in-biotechnologytechnologytransfer-to-2014-deal-volume-has-doubled-sin/

[104] http://www.eunethta.eu/about-us/faq\#t287n73

[105] http://ec.europa.eu/eahc/health/tenders H09 2013.html

[106] http://www.cdc.gov/genomics/gtesting/ACCE/index.htm

[107] http://www.egappreviews.org/about.htm

[108] http://www.htai.org/index.php?id=420

http://cmsserv.ncat.edu/doclib/DORED,\%200utreach\%20and\%20Technology\%20Transfer\%20(How\%20T o\%20Section)\%20Web\%20site,-5.pdf

[110] http://www.pkdcure.org/learn/healthcare-professionals/drug-development-timeline-infographic

\section{Acknowledgements}

This project is supported by a grant from the European Commission PHGEN II (Duration period: June, 2009-November 2012 EU-Project No. 20081302). This work is also supported by the Canadian Institutes for Health Research (CIHR), CIHR Institute of Genetics, the CIHR Institute of Health Services and Policy Research through a grant (no. ETG92250) to the APOGEE-Net/CanGèneTest Research and Knowledge Network on Genetic Services and Policy. We also like to extend our thanks to the research school GROW (program Public Health Genomics) of the Faculty of Health, Medicine and Life Sciences of Maastricht University for supporting this research. 
CHAPTER III

THE LAL MODEL: FROM THEORY TO HYPOTHESIS OF STEPS FOR IMPLEMENTATION OF BASIC GENOME-BASED EVIDENCES IN PERSONALIZED MEDICINE

Published as:

Lal JA, Vaidya A, Gutiérrez-Ibarluzea I, Dauben HP, Brand A. Personalized Medicine 2013, 10(7):683-701. 


\section{Abstract}

We see a backlog in the effective and efficient integration of personalized medicine applications such as genome-based information and technologies into healthcare systems. This article aims to expand on the steps of a published innovative model, which addresses the bottleneck of real-time integration into healthcare. We present a deconstruction of the Learning-Adapting-Leveling model to simplify the steps. We found out that throughout the technology transfer pipeline, contacts, assessments and adaptations/ feedback loops are made with health needs assessment, health technology assessment and health impact assessment professionals in the same order by the academic-industrial complex, resulting in early-on involvement of all stakeholders. We conclude that the model steps can be used to resolve the bottleneck of implementation of personalized medicine application into healthcare systems.

\section{Keywords}

Health Impact Assessment, Health Needs Assessment, Health Technology Assessment, LAL Model, Life Cycle, Personalized Healthcare, Personalized Medicine, Public Health Genomics, Technology Transfer, Translational Research. 
Through scientific and technological advancement, we have come to know the dynamically complex nature of human molecular biology [1-8]. Furthermore, epigenomics [9] and pharmacogenomics [10] have demonstrated that individuals are unique and may respond differentially to a lifestyle change, treatment or disease. The interactome, which is the complete repertoire of interactions potentially encoded by the genome [11], indicates a more unique or personalized reaction to the exposome, which is the combined exposures from all sources that reach the internal chemical environment [12]. Since the post-genomics era and the inception of systems biology and systems biomedicine [13], we have seen a shift towards the concept of 'one shoe does not fit all' [14] as every individual is unique. This has made us reconsider our approaches to health. With the advent of social media and access to the internet, a trend is being seen by patients not wanting to be treated collectively, but in a more personalized or individualized manner [15]. As a result, technological innovation in the form of computational power, including systems biology coupled with wet laboratories, has driven genome-based information and technologies (GBITs) to push the agenda of healthcare systems to personalized medicine. Consequently, personalized medicine refers to the use of genome-based information and technologies for providing more stratified and precise (and possibly truly personalized) interventions [16] to individuals. 'Genomebased' encompasses all omics deriving from the human genome. Technologies include technologies in the general sense, as well as techniques, processes, methodology, diagnostic kits, applications, interventions (including drugs) and activities [17]. Genome-based information also constitutes omics interactions with the environment [18]. The environment not only embraces the location and exposures to known and unknown toxic agents, and climate, but also lifestyle and socioeconomic factors. These GBITs give true meaning to personalized medicine, as omics and other unique data retrieved from an individual combined with computational modeling bring a sense of individualized medicine [16]. In other words, GBITs have helped propel, as well as clear, the information cloud mentioned above. As a result, GBITs have brought discovered treatment options to a more personalized level. Therefore, in this article, the focus is not just on translating genome-based technologies [17] into personalized medicine, but also on the translation of GBITs.

As promising and exciting as personalized medicine may be, we see a lack of, or delay in translation of these GBITs into healthcare [17]. This is due to the lack of a cross-cutting integration of different and 


\section{| CHAPTER III}

related concepts/tools [19] for translation and implementation. These different and yet related tools address different and/or similar or overlapping aspects of the various stages of translation and implementation but have not been used with an integrative approach. This issue also occurs in many other types of health technologies. As a result, manufacturers of technologies and systems are pushing for an early dialog among the different parties involved in translation and implementation [101]. Therefore, a need exists for innovative translational models to bring personalized medicine research into healthcare systems through relevant GBITs. This article aims to demonstrate the steps of such a model.

In 2011, we published the Learning-Adapting-Leveling (LAL) model [17], in which we addressed the bottleneck of effective and efficient real-time uptake of relevant GBITs by healthcare systems. We identified the non-synergy or non-alignment between the academic-industrial complex [20] and policymakers that mostly run in parallel (at different timeframes of GBIT development) with no interaction among them. Consequently, we stated that technology transfer $(T T)$ and the public health assessment tools (PHAT) are the authoritative tools or activities used by the academic-industrial complex and policy/decision-makers, respectively $[17,18]$. $T T$ is the translation of research ideas from the laboratory through the industrial application onto the market. $T T$ can be defined as the migration of academic discoveries to useful applications in the development of marketable products or processes [21]; in this case, GBITs. On the other hand, PHAT correspond to implementing GBITs from the market into healthcare, hence its integration. This includes aspects of reimbursement, quality assurance, policy, recommendations and best practice guidelines as well as decision-making. PHAT include health needs assessment (HNA), health technology assessment (HTA) and health impact assessment (HIA). In simplistic terms, HNA identifies the needs of the population and takes into account the characteristics of the systems in order to allocate resources accordingly. HTA assesses the performance of healthcare technologies including their ethical, legal, organizational, economic and social implications. HIA on the other hand, assesses the effects of policies, programs or projects (including technologies) on the population's health [22]. The collective PHAT (HNA, HTA and HIA) are used by decision-makers (policy) based on reports from these three different assessment domains and working organizations. These recommendations, among others (opportunity is also part of the game), enable policy development, which in turn affects the wide usage and acceptance of GBITs in hospitals, healthcare systems and public health in general. These political decisions will determine the final outcome of treatment in patients and citizens, as well as the burden of disease; for example, reimbursement and insurance premium will be determined through these decisions, guideline and protocol development, release of government 
funding and restructuring of systems to accommodate these technologies, among others. On the other hand, if a relevant technology that can reduce the time to diagnosis and prognosis is rejected, it could possibly lead to higher costs as late diagnosis and prognosis will require more expensive treatments compared with early diagnosis and prognosis leading to prevention. This affects the profit margin of industry, as well as patients willing to receive relevant GBITs for treatment, in a timely manner. Similarly, from a different perspective, if rejection of the GBITs is not done, its inadequate inclusion itself can result in incorrect wide and early adoption yielding similar results as mentioned above.

The LAL model is hypothesized to be a possible solution to this bottleneck as at its base lies the pseudoparallel initiation of previously non-communicative TT and PHAT giving rise to cross-talk, public-private partnerships (PPP) and consultation in order to create a networking and crossing exercise [17]. As a result, participation of all stakeholders in the value chain of the technology early on in its development brings in a so-called win-win process $[17,18]$. Stakeholders include, but are not limited to, HTA professionals, insurance companies, healthcare providers, health professionals, patient groups, citizens, venture capitalists, decision-makers, industry and academics, among others. TT and PHAT through the process use the Public Health Genomics (PHG) wheel [23] as a reference to ensure that any possible overlooked gaps are addressed [17] through the ten essential public health tasks, which integrate GBITs in healthcare at its different levels. This affirms that by the time the technology is developed, it conforms to the standards required by decision-makers, possibly preventing an eventual lag in uptake. The LAL model includes feedback loops and is dependent on the value of information (Vol) or quality of evidence (QoE). The Vol determines the understandability of the technology to the end-user, and its relevance and limitations in terms of patents, among others, for wide usage. The LAL model works around this issue, thereby giving recommendations. In summary, the LAL model can be seen as a framework to assess the feasibility of the developing technology from the start to near-end of the technology maturation process for real-time uptake by healthcare systems and policy guidelines. Consequently, the model develops real-time recommendations to compensate for any gaps in the process prior to the launch of the product through contact with policy [17] by building a network. This ascertains that by the time the technology is ready, it can be immediately taken up by healthcare. Recently, we have argued the case for the LAL model as the required cross cutting [19] overarching framework for the effective and efficient integration of relevant genome-based technologies into healthcare systems [18] and also given its integration in best practice guidelines [24] and other running EU grants, small medium enterprises (SMEs) and consortia $[102,103]$. In addition, it is much more structured compared with other existing 


\section{| CHAPTER III}

initiatives of early dialog; for example, the Green Park Collaborative [104], which defined just outcomes of interest that will be used in future steps of the life cycle of a technology. As a result, we believe that a higher order structured model, such as the LAL model, can offer an advantage over simpler models.

The unique case of the LAL model brings together, for the first time on the same platform, the academic-industrial complex (through TT) and decision-makers (through PHAT) $[14,17,18,25]$. Platform, in this case, is the common grounds or table on which these stakeholders are brought to cross-talk; in other words, providing a stage for cross-talk and bilateral communication. In this article, we aim to develop an adapted yet flexible step-by-step methodology (toolkit) for the framework regarding how to use the LAL model. This, we believe, can eventually help in providing valuable information and to pilot in a concrete subset of health technologies that are GBITs. These developed steps can be useful for academics, industrial partners and decision-makers alike, as well as patient groups, in order to advance timely uptake of relevant GBITs benefiting all. It could also be a useful framework for other types of health technologies that experiment with a stop in their development and subsequent uptake by healthcare systems, but fine tunings may be needed to adapt it to these specific technologies. 


\section{Methodology}

The method used to describe the step-by-step guide of the LAL model will be the deconstruction of the LAL model itself [17] as can be seen in Figure 1. We will start with the elaboration of the reference frames (the Vol and PHG wheel). Vol will be touched upon from two perspectives; business and the HTA point of view. We will then go through the pseudo-parallel initiation of $\pi$ and the PHAT in the innovation network. Pseudo-parallel indicates a sense of parallel flow, however may not be absolute. Furthermore, $T$ initiates a bit earlier than PHAT (Figure 1). The deconstruction of the steps of the pseudo-parallel initiation of TT and PHAT will be based on the development of the innovation network (Figure 1) through the steps below [17]:

- Identification of the health need (market pull/push) by industry and subsequent research and development. We mark this as $0 \%$ innovation network;

- After initial research and development, contact should be made with the relevant authorities in HNA/HTA and policy. We consider this $10 \%$ development of the innovation network, which starts from $0 \%$;

- A proposal is developed either jointly (PHAT and industry) or individually (e.g., academiaindustry) and it should be advocated. Networking is the key here. We consider this $20 \%$ development of the innovation network;

- Initiation of PPP of the industry with policy through the PHAT authorities. Partnership can be in terms of collaborations, knowledge sharing and joint investigations, among others. This is a major milestone; therefore, we consider this $40 \%$ capacity of the innovation network;

- Over time, the innovation network will be developed and expanded as the industry collaborates with decision-makers through the process of the PHAT and $\Pi$ activity pipelines, which can look like a pseudo-merger. We consider this $60 \%$ network capacity;

- Finally, the innovation network should have matured enough to integrate relevant technologies into healthcare systems and policy in real time as a consequence and evaluate the impact using HIA. We believe the innovation network is at $80 \%$ functionality and is considered the threshold of the absorption capacity of this apparatus. 


\section{| CHAPTER III}

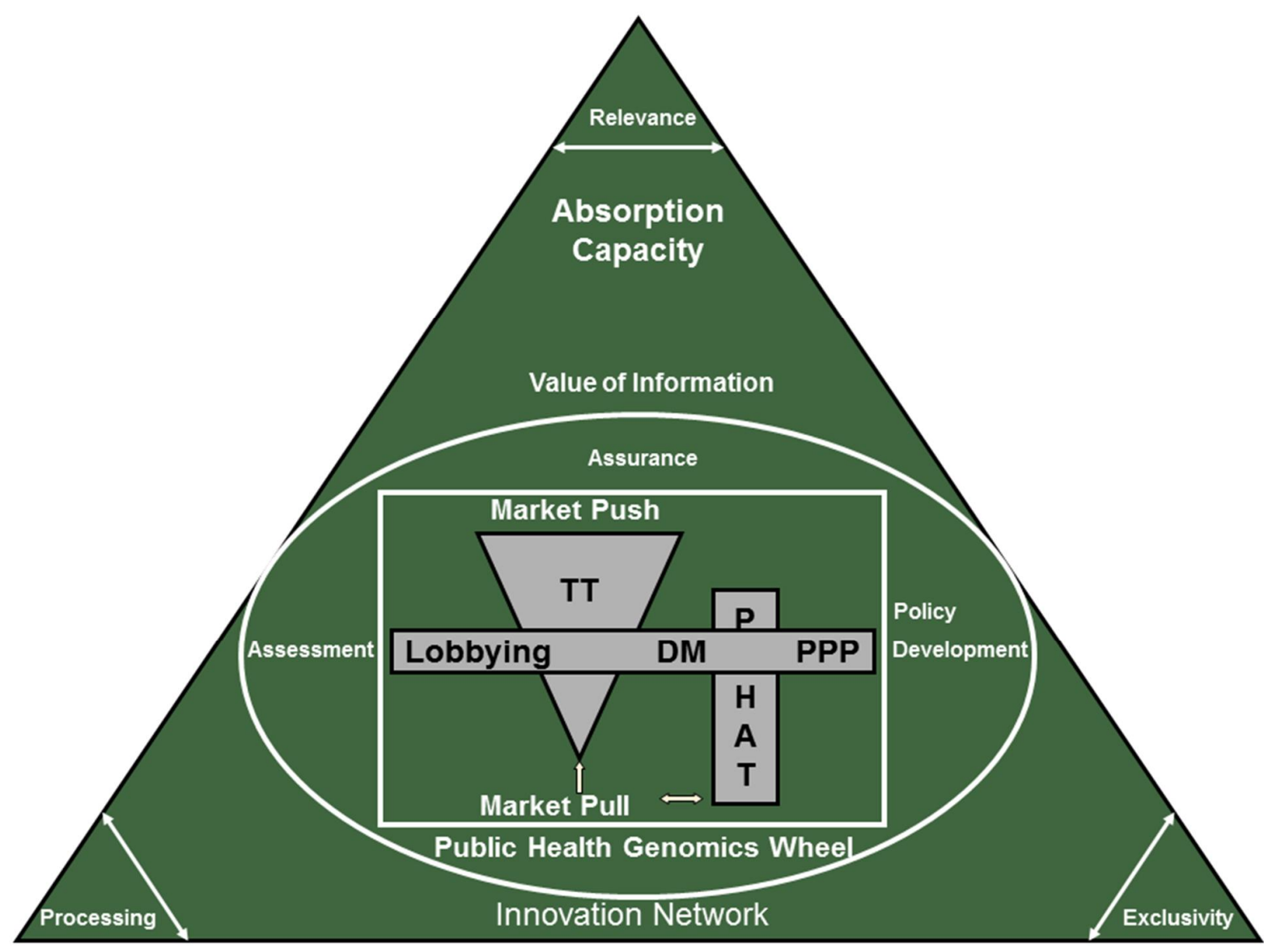

Figure 1: The Learning-Adapting-Leveling model. The distinct feature of the model is at its very heart: it brings together for the first time TT (used by the academic-industrial complex) and PHAT (accessed by DMs) to bilateral communication, resulting in an (innovation) crossing. The idea is, that through this two-way communication (ad hoc crossings), professionals on both sides can cooperate and participate in the development of a new genome-based information and technology for the academic-industrial complex, resulting in conformity to previously unmet healthcare needs and also discern the relevant technology based on the needs of the population identified by DMs (via PHAT). In other words, early involvement of all stakeholders ensures, through interaction and participation, that the emerging technology answers all questions required, and if not, it is adapted accordingly or dropped, thereby restarting (or continuing in parallel) on an alternate. This is an iterative activity involving pushing the agenda of relevant technologies (lobbying), resulting in possible broadly defined PPP [17] (see pages 11-13 in this reference). The preferred sequence of events in the PHAT are in the order stated viz. health needs assessment, health technology assessment and health impact assessment, respectively, although this is not absolute. Through the flow of work between PHAT and TT, the PHG wheel, via its ten essential public health tasks [23] distributed over the domains of assessment, policy development and assurance, is used as a reference frame to ensure all possible gaps in healthcare integration are addressed. In parallel, the value of information or quality of evidence with respect to the technology's 'relevance' (added value) to the population or end-user (e.g., health professionals, managers and patients, among others) including its affordability (economic, ethical, legal, social and organizational) by the systems, its 'processing ability' in terms of understandability or ease of use to the handler/user, and 'exclusivity' resulting in restriction of usage due to patent issues, among others, are addressed, thereby solutions can be developed from them. The value of information and public health genomics wheel ensure that all gaps and issues are dealt with at an early stage of technology development. $T T$ is symbolically an inverse triangle as there is a large amount of data generated initially in research and development and, from that data, it narrows down to a few options through the TT pipeline to innovate or valorize. During this initial stage it is hard to compute with 
PHAT, hence TT's first research and development starts slightly earlier than PHAT (see inverse triangle to PHAT block). The (market or population) need is the driving force behind both TT and PHAT. These bring in the concepts of market push and pull of a relevant technology as TT uses both in its strategy and PHAT evaluate the market pull and investigate a technology to (market) push, based on population needs. The absorption capacity is the optimal threshold of the innovation network achieved over several iterations of this PPP.

DM: Decision-maker; PHAT: Public Health Assessment Tool; PPP: public-private partnership; TT: Technology transfer. Reproduced with permisson from [17].

Where required, further description of a concept will be emphasized, provided it has not been mentioned in the introduction section above or is new. 


\section{Results}

The LAL model is named as such because within the model, the stakeholders learn (from each other), adapt (to each other's requirements and conform within their organization) and level (or even the modes of communication for understanding and adapting through these widely defined PPPs). Therefore, iterations and feedback loops are primarily involved in this model through the innovation network.

As stated in the LAL model, there are two reference points; one being the Vol and the other being the PHG wheel. When talking in terms of Vol, it can be seen from two different perspectives with both simultaneously applied within the LAL model as reference frames. The first being the Vol from a business perspective and the other being Vol from an HTA perspective. When speaking in terms of business, Vol with its three pillars (Figure 1) of relevance, processing ability and exclusivity tends to incline towards a more customer-centric model or end-user-centric model. This end-user-centric model is in the form of customer value management (CVM) or end-user value management. Adapted [26], CVM is managing each end-user relationship with the goal of achieving maximum lifetime profit from the entire user base. When using Vol in the form of CVM, we believe the following questions need to be taken into consideration [26] while developing the technology and if possible even before:

- Question 1: Is CVM being used to improve business performance?

- Question 2: Is the current model of CVM more end-user driven rather than IT driven?

- Question 3: Is customer lifetime value (CLV) being used as an important core measuring tool? ff

- Question 4: Are competing technologies and context-dependent organizational frameworks considered when analyzing the CVM?

- Question 5: How strong are our analytical capabilities?

- Question 6: Are the drivers of customer acquisition, retention and expansion recognized?

- Question 7: Are we creating customer value in the currently managed communication channels?

The seven questions above are the guiding tools for the business side of the Vol with question 1 within the 'exclusivity' pillar and 2-5 within the 'relevance' pillar. Questions 6 and 7 are part of the 'processing ability' pillar. According to Verhoef and Lemon [26], if the above questions are answered in the positive or adapted to answer in the positive, it can help in giving an advantage over the competition. This is due 
to the fact that these questions develop the business plan towards an end-user-centric concept. These plans can be provided through a concrete analytical capability. This capability is defined as the process of extensively employing data, quantitative analysis, statistical models and fact-based management techniques to drive firm decisions and actions [26]. Furthermore, these plans can be evaluated through CLV. CLV is defined as the net present value of all future profits derived from a customer over his/her lifetime with the business [26]. When businesses or SMEs have a more end-user-centric model resulting in investment in customer (end-user in our case) intelligence $(\mathrm{Cl})$, it has been demonstrated to give an advantage in the market [26]. $\mathrm{Cl}$ is the process of gathering and analyzing information regarding customers, their details and their activities, in order to build deeper and more effective customer relationships and improve strategic decision-making [105]. Answering the above seven questions positively has been demonstrated to give better outcomes in profit and success. Verhoef and Lemon [26] provide an excellent (review/research) article demonstrating this aspect. The article mentioned demonstrates that these questions should not be answered simply in the 'yes or no' format, but rather in more detail describing what steps have been taken to adapt the answers to yes.

Therefore, the seven questions are one aspect of the reference frame of the LAL model's (business) Vol, answered simultaneously with HTA's Vol. In this sense, we have taken the liberty of deriving a table summary from Verhoef and Lemon [26] per derived question above and its approaches in the format of adapted sub-questions as given in Box 1. If TT goes through these questions and acts upon them, it can ensure that their product is well received by the end-user.

On the other hand, we have Vol from the perspective of HTA. It is to be noted here that within the core of the LAL model, Vol is assessed during the HTA step (described in the subsequent paragraphs). However, we believe a shorter version of this HTA's Vol should be kept as a reference frame prior to the start, as well as in the process itself, to prepare oneself for adaptations or loose ends. HTA-based decisions to adopt a technology always pose the question of whether the evidence base to support this decision is sufficient. Vol analysis provides an analytical framework to determine the value of expanding the evidence base to inform a decision problem by performing additional research. Vol from the HTA perspective involves decision analysis regarding the commitment of resources for future research in order to minimize uncertainty surrounding the expected benefit from a new technology and to maximize the health gain. A framework has been published by Claxton et al. to compare the potential benefits of further research with the costs of further investigation, a comparison and prioritization of alternative 


\section{| CHAPTER |||}

research recommendations, as well as an assessment of the value of investing resources in research or other activities, such as the provision of health service [27]. The expected Vol for a technology is simply the difference between the expected value of the decision made with perfect information about the uncertain parameters and the decision made on the basis of existing evidence. Vol is further described later in this article under the heading of 'innovation network'. This perspective should be looked upon by both TT and PHAT prior to and during the process of the LAL model to meet head-on on issues coming up as a consequence.

Box 1: Summary of approaches per question with respect to customer value management. CLV: Customer lifetime value, CVM: Customer value management, ICT: Information and communication technology. Data taken from [25] with exception of question 4.

\begin{tabular}{|c|c|}
\hline $\begin{array}{l}\text { Question } \\
\text { No. }\end{array}$ & Approach \\
\hline 1 & $\begin{array}{l}\text { - Does it give an improved competitive advantage? } \\
\text { - Does it increase the company's end-user centric model? } \\
\text { - Does it increase marketing accountability? }\end{array}$ \\
\hline 2 & $\begin{array}{l}\text { - Is the focus more internal than external? } \\
\text { - Does the current ICT facilitate the management of end-user relationship? } \\
\text { - Is the focus on the underlying value of the enhancement rather than the enhancement } \\
\text { itself? } \\
\text { - Is the technology investment benefiting the end user and their management? } \\
\text { - Is there an end-user strategy before implementation? } \\
\text { - } \quad \text { Is your organization counter-productive to customer (end-user) orientation by being } \\
\text { - How much employee level involvement exists with end users? } \\
\text { - Do all departments have defined responsibilities within the customer centric model? } \\
\text { - } \quad \text { Are customer profitability and customer satisfaction taken into account? }\end{array}$ \\
\hline 3 & $\begin{array}{l}\text { - Are sales promotions decreasing customers by increasing consumer price sensitivity? } \\
\text { - Is the interdependency of purchase behavior within product categories defined? } \\
\text { - Is the focus too much on a single product option? } \\
\text { - Are there strategies focused on maximizing CLV? } \\
\text { - Can you evaluate or interpret investments and marketing strategies using CLV thus increase } \\
\text { marketing accountability? }\end{array}$ \\
\hline
\end{tabular}




\begin{tabular}{|c|c|}
\hline & $\begin{array}{l}\text { - Can you determine the value of your user base? } \\
\text { - If so, can you link their equity to shareholder value? } \\
\text { - Is segmentation and resource allocation with end users plausible as a result? } \\
\text { - Is the end-user portfolio dependent on CLVs? } \\
\text { - Are bonuses and incentives based on evaluation of CLVs? }\end{array}$ \\
\hline 4 & $\begin{array}{l}\text { - Do you have knowledge of the organizational and structural conditions of the end user? } \\
\text { - Have you taken into account the influence of competing technologies in the end user } \\
\text { perception of the value of the technology? } \\
\text { - How could competing technologies and the perceptions of managers' influence future } \\
\text { pricing and reimbursement? }\end{array}$ \\
\hline 5 & $\begin{array}{l}\text { - Do you have an end-user oriented analytical based selection strategy? } \\
\text { - Do you use fact based management techniques? } \\
\text { - Do you heavily rely on customer intelligence? } \\
\text { - If so do you use cross-tabulation, tree-based methods, logistic regression and/or boosting } \\
\text { - techniques? } \\
\text { - Does your company also have a customer intelligence marketing interface to translate the } \\
\text { - Does your customer intelligence have interdisciplinary functions? }\end{array}$ \\
\hline 6 & 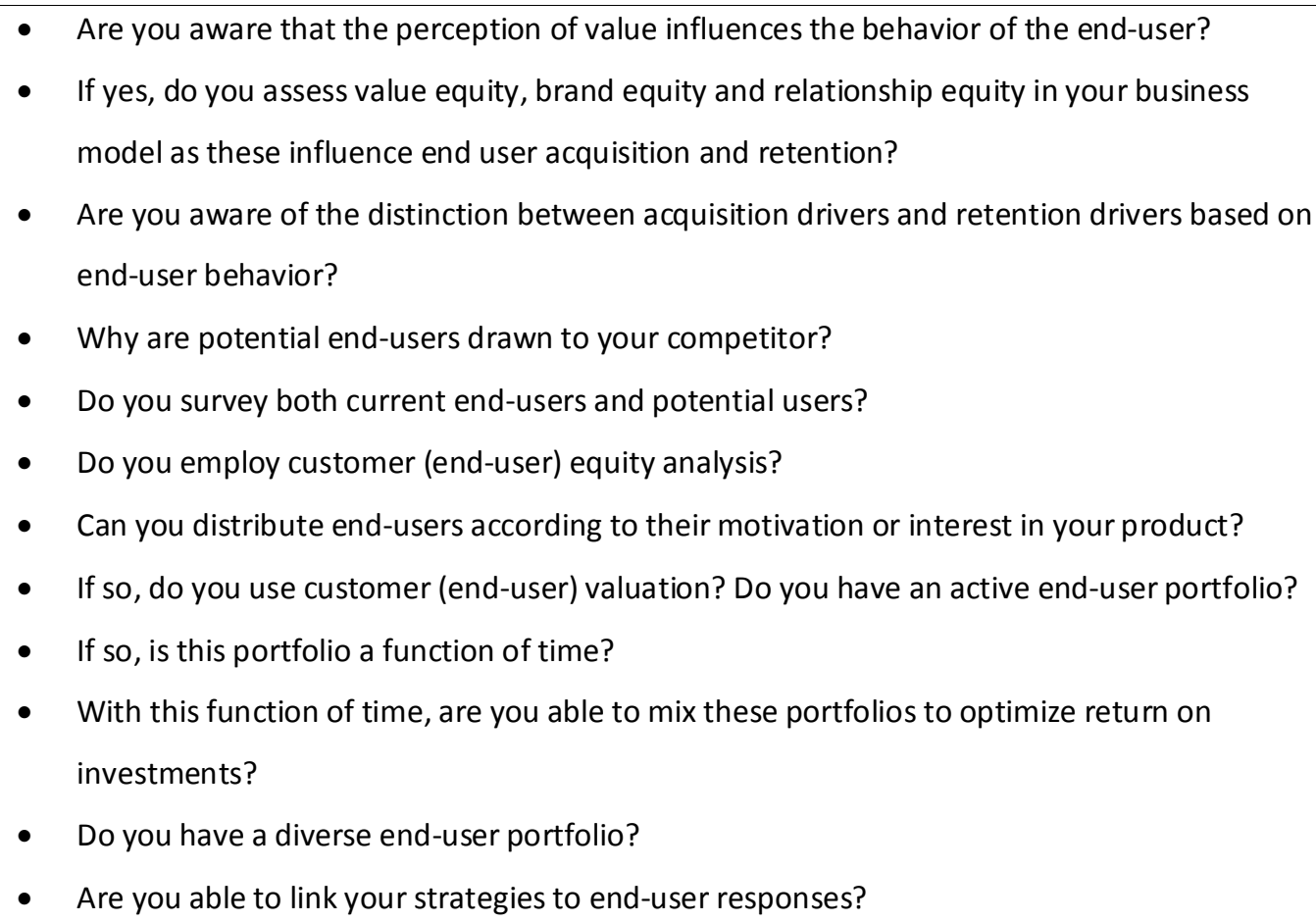 \\
\hline 7 & - Is customer value maintained as well as created in the diverse communication channels you \\
\hline
\end{tabular}




\begin{tabular}{|l|l|}
\hline use? \\
- Having multiple channels make things more complex or valuable? \\
- Are your end-users lost in translation by these multiple channels? \\
- Are you forcing end-users to a specific channel? \\
- Do you have a feedback process on multichannel usage? \\
- Are you able to allocate resources efficiently over these channels?
\end{tabular}

The other reference frame is the PHG wheel [23] with its domains of policy development, assurance, assessment and reassessment (possible disinvestment or deprescription) defining the ten essential tasks as given in Table 1. These tasks fulfill the public health integration. The idea behind this reference frame of the PHG wheel is to cross-check these tasks for possible issues within each domain, which may need to be taken into consideration for the specific technology in development for acceptance in public health and healthcare. Although these topics are touched upon in the PHAT [18], like Vol, these are good reference points to be taken into consideration based on the relevance of the reference point to the applicability of the technology. Noticeable is that the application of the reference points of Vol and the PHG wheel have direct implications on the development of the innovation network (Figure 1). If one goes through the processes involved in either reference frame, it inevitably gives rise to the innovation network. The reference frames therefore are a prelude to the actual development of the innovation network through broadly defined PPPs [17] materialized through the core interactions between TT and PHAT. Importantly, the reference frames are to be kept in parallel as a checklist prior to starting the LAL model and during the LAL model (when TT and PHAT have been initiated). This way, overlooked issues, both from business development and healthcare integration, as well as public health perspectives, are brought up and addressed. The PHG wheel does not have a defined methodology, but rather defines the tasks to be addressed. The PHAT resolve or define this in its own methodology [18]. On the other hand, in the case of Vol, it is possibly addressed before or during the development of the technology. It is essential to state here that the reference frames are not themselves the core steps within the LAL model but can be considered a pre-step or a continued step, or as a checklist for which to prepare.

We have defined and described how to use the reference frames prior to and while embarking on the core of the LAL model. As stated in the LAL model article $[17,18]$, and based on Figure 1 , we will now present our step-by-step method on how to use the framework. Some assumptions have to be stated. The ideal standard for an idea to develop and reach the market is 5-10 years (innovation pipeline) on 
average. This ideal standard is based on the time to market, given a reasonable return on investment [28], as well as being based on our own experience. So we will keep the timeframe of the usage of the LAL model for 5 years in the current example, although this may vary depending on when the technology reaches the market and can give some room for the analysis. Our steps are summarized in Figure 2.

Table 1: Details of the (ten) essential tasks of the domains of assessment, policy development and assurance of public health. GBIT: Genome-based information and technology. Adapted with permission from [22] with findings taken from [23].

\begin{tabular}{|c|c|}
\hline $\begin{array}{l}\text { Monitor Health } \\
\text { Diagnose \& } \\
\text { investigate }\end{array}$ & $\begin{array}{l}\text { Core function } \\
\text { The regular systematic collection, assembly, analysis and dissemination of personal } \\
\text { health information including GBITs } \\
\text { Related essential services } \\
\text { - Basic research: quantifying the impact of GBITs and their interaction with } \\
\text { environmental factors including knowledge from epigenomics, microbiome } \\
\text { etc. } \\
\text { Monitoring health: monitoring health status, including genome-based } \\
\text { factors, to identify health problems within the healthcare system. } \\
\text { Diagnosing and investigating: investigating the distribution of genome-based } \\
\text { and modifiable risk factors to determine their contribution to identified } \\
\text { health problems and to improve health outcomes. }\end{array}$ \\
\hline $\begin{array}{l}\text { - Inform, } \\
\text { educate, } \\
\text { empower } \\
\text { - } \text { Mobilize } \\
\text { community } \\
\text { partnerships } \\
\text { Develop } \\
\text { policies }\end{array}$ & $\begin{array}{l}\text { Core function } \\
\text { The formulation of standards and guidelines, in collaboration with stakeholders, } \\
\text { which promote the appropriate use of GBITs and the effectiveness, accessibility and } \\
\text { quality of their tests and services. } \\
\text { Related essential services } \\
\text { - Policy and communications research: identifying and analyzing the economic, } \\
\text { social, ethical and political implications of advances in GBITs, including the } \\
\text { information and communications needs of stakeholders. } \\
\text { Informing, educating, empowering: facilitating communications and } \\
\text { education about the integration of GBITs into health promotion and disease } \\
\text { prevention programs. } \\
\text { Mobilizing partnerships: fostering collaboration between public and private }\end{array}$ \\
\hline
\end{tabular}




\begin{tabular}{|c|c|}
\hline & $\begin{array}{l}\text { agencies and constituent groups to promote effective and efficient } \\
\text { communication and policy making about GBITs. } \\
\text { Developing policies: establishing standards and guidelines for when and how } \\
\text { genetic information should be applied to promote health and prevent } \\
\text { disease. }\end{array}$ \\
\hline $\begin{array}{ll}\text { - } & \text { Enforce laws } \\
\text { - } & \text { Link to/ provide } \\
\text { care } \\
\text { - } \\
\text { Assure } \\
\text { competent } \\
\text { workforce } \\
\text { - } \quad \text { Evaluate }\end{array}$ & $\begin{array}{l}\text { Core function } \\
\text { Assuring constituents that GBITs are used appropriately and that tests and services } \\
\text { meet agreed-upon goals for effectiveness, accessibility and quality. } \\
\text { Related essential services } \\
\text { - Health services research: identifying and analyzing the factors that influence } \\
\text { the impact of GBITs and the delivery, utilization and quality of health } \\
\text { information and services. } \\
\text { Enforcing laws: promoting the enforcement of policies and standards } \\
\text { enacted to ensure the appropriate use of GBITs and the effectiveness, } \\
\text { accessibility and quality of genome-based (health) information and services. } \\
\text { Linking to/providing care: ensuring the availability and accessibility of GBITs } \\
\text { and services and associated interventions to improve health and prevent } \\
\text { disease. } \\
\text { - Assuring a competent workforce: ensuring that present and future health } \\
\text { professionals have training and skills in the appropriate use of GBITs to } \\
\text { promote health and prevent disease. } \\
\text { and quality of this information and related services. }\end{array}$ \\
\hline System Management (1) & $\begin{array}{l}\text { Building and maintaining the capacity of the public health infrastructure to integrate } \\
\text { GBITs into public health policy, research and practice. }\end{array}$ \\
\hline
\end{tabular}

As can be seen from Figure 2, TT starts a bit earlier than PHAT (Figure 1) as the latter's HNA cannot identify with the early stages of the current research [17]. Initial work starts with basic research (and preclinical research and development) which can be motivated by the need (market pull/push) in the market (Figure 1). The pseudo-parallel initiation is from the concept of broadly defined PPPs [17], which bring in the early-on involvement of all relevant stakeholders. From our previous article [17], we hypothetically demonstrated the steps involved in the development of the innovation network given in the methodology section above. In the subsequent paragraphs, we will expand on these steps. It is to be 
noted that feedback loops and iterations are commonly based on the response of either party. Furthermore, the reference points (Vol and PHAT) are cross-checked for applicable areas possibly overlooked throughout the whole innovation pipeline (0-80\% innovation network). These two important points may or may not be mentioned again for the sake of simplicity, however, should be kept in mind at all times. Please refer to Figures $1 \& 2$ through the steps below.

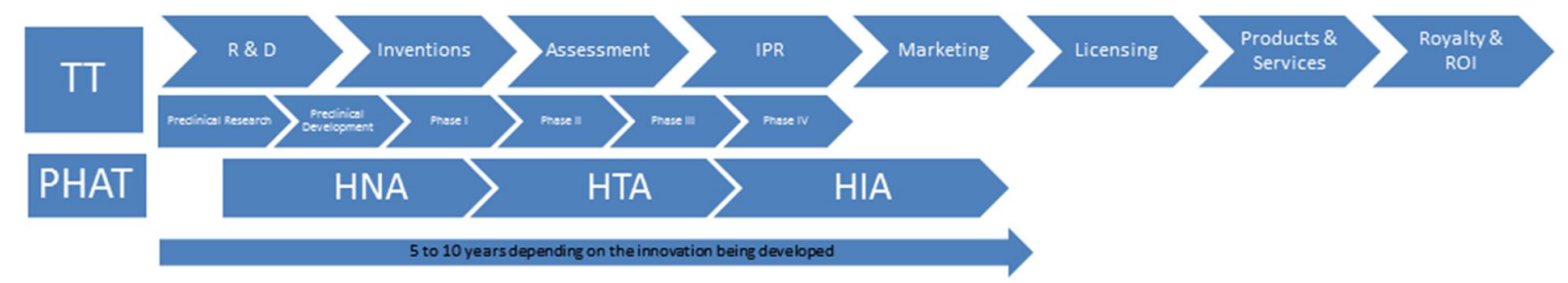

Figure 2: Summary and breakdown of the pseudo-parallel initiation of technology transfer and the public health assessment tools. The first two rows belong to $\Pi$ and the third row belongs to PHAT. The fourth row gives an estimation of the duration of the parallel initiation (TT and PHAT). The first row subjectively indicates the complete TT activity [107]; the second row indicates the US FDA's view on drug development, which is within the boundaries of $\Pi$, of which the parallel is shown [108]. TT starts with basic R\&D in the laboratory which leads to an idea of an application, which develops into an invention or conceptual illustration. This invention is assessed for its feasibility for market introduction including strength, weaknesses, opportunities and threats and qualitative research analysis. If the assessment proves worthwhile to pursue, prior to market introduction, the invention or product's intellectual property is protected giving it IPR and market approval is sought during or after the assessment. Depending on whether the invention is a small medium enterprises product and/or from a larger established organization the product can be licensed for mass production or sales, which results in new products and services. Consequently, the licensed products generate possible profits which give return on investment by the stakeholders and payments on royalties. In the FDA's view, Phase I concerns testing the drug for safety, administered amount and side effects in a volunteered small healthy group of subjects [108]. Phase II is concerned with effectiveness, as well as safety, in a small healthy group of volunteer subjects. Phase III involves testing the drug on a large group of volunteer subjects. This is to establish the effectiveness, as well as to observe side effects and compare with currently used treatments. Phase IV is carried out when the drug is approved and is being used. The concept is to gather and analyze data on risks, benefits and best usage. HNA (1 year) identifies the required needs of the population and allocates resources accordingly. HTA (3 years) assesses the performance of healthcare technologies, including ethical, legal, economic, organizational and social implications. HIA (2 years), on the other hand, assesses the effects of policies, programs or projects on the population's health [22].

HIA: Health impact assessment; HNA: Health needs assessment; HTA: Health technology assessment; IPR: Intellectual property right; PHAT: Public health assessment tool; PPP: Public-private partnership; R\&D: Research and development; ROI: Return on investment; $\Pi$ : Technology transfer. 


\section{| CHAPTER |||}

\section{Part 1: $0 \%$ innovation network}

Market dynamics are driven by the need in the market. This (health) need can either be created (market push) or can capitalize on existing demands (market pull). The academic-industrial complex [20] is at the forefront in identifying or developing the need; however, the healthcare system itself can sometimes identify the need, depending upon patient/healthcare need. These healthcare system-identified needs are selected through early awareness and alert systems including strengths, weaknesses, opportunities and threats, and qualitative research analysis [29,30]; however, we believe true early awareness and alert systems are not fully realized [18] as healthcare is in reach with only upcoming technologies in the public domain to assess. Coming back to the academic-industrial complex, the market health need is the driving force behind research (including preclinical research). The academic-industrial complex keeps in mind the Vol and PHG wheel and tries to conform to the developmental process of their research to the references while in these stages. As can be seen from Figure 2, during the initial research, HNA cannot identify with the preliminary ongoing research as it is in its initial stages and promising applicability cannot be determined. This is considered as $0 \%$ of the innovation network as no communication has been made with healthcare policy.

\section{Part 2: 10\% innovation network}

During the different phases of research and development (Figures $1 \& 2$ ) and in the case of drug development, the latter phases of preclinical research and the different stages of preclinical development including initial stages of Phase I, initial contact followed by multiple contacts, will be made with HNA. This is the start of the broadly defined PPPs [17] of the innovation network. Based on the requirements of the reference frames and the current identified need, feedback is sought based on the relevance of the solution. A preliminary HNA assessment will be carried out, which will bring the TT people back and forth to the drawing board to conform to the identified health need. $\pi$ will conform according to the given information, as conformity will ensure acceptance of their technology to healthcare systems, thus possibly generating profit.

The need from this perspective can be defined as 'the ability to benefit from the intervention' [31]. The availability of an intervention may not necessarily indicate a need; similarly a demand for an intervention may not indicate a need. The need for healthcare must be distinguished from the need for health. The need for healthcare is much more specific than the need for health and is now widely accepted to mean 
the population's ability to benefit from healthcare. It depends on the potential of preventive or treatment services to remedy health problems.

HNA, therefore, is a systematic method of identifying unmet health and healthcare needs of a population and making changes to meet those unmet needs. It provides information for:

- Improving health;

- Service planning;

- Priority setting;

- Policy development.

HNA is thus not a health status of population assessment. It aims to improve health and it incorporates the concept of a capacity to benefit from an intervention. HNA usually aims to make incremental changes to existing services [106].

HNA is performed by combining epidemiological approaches with patient's perspectives, assessment of the effectiveness and possible cost-effectiveness of interventions. The principal activities involved in healthcare needs assessment are therefore:

- The assessment of incidence and prevalence (how many people need the service/intervention);

- The effectiveness and cost-effectiveness of their services (do they confer any benefit, and if so, at what cost, i.e., what is the relative benefit?) and the baseline services.

During the process of HNA, the following questions are answered;

- What is the problem?

- What is the size and nature of the problem?

- What are the current services?

- Identify interventions by asking patients what they want;

- Identify interventions by reviewing scientific knowledge;

- Consult professionals and other stakeholders;

- What are the most cost effective solutions?

- What are the resource implications?

-What are the recommendations and plan for implementation?

- What are the outcomes to evaluate change? 


\section{| CHAPTER |||}

Subsequently, initial contact is made with HTA professionals during or after adaptation has been performed based on the HNA assessment. The idea is to get them in touch with a prospective upcoming technology which is not currently in the public domain for the HTA's early awareness and alert systems bringing the concept of true alert systems and constructive technology assessment (CTA) [18,32,33]. This is during the near-end of the invention phase (Figure 2) and also includes Phase I of drug development. This is considered $10 \%$ of the innovation network.

\section{Part 3: $20 \%$ innovation network}

This involves the near-end of the invention development including the assessment for feasibility in the $\Pi$ pipeline (Figure 2) and the start of patenting (intellectual property rights). This also includes Phase I through Phase III of drug development. The HTA professionals assess (possibly via horizon scanning or CTA) [34] this prospective technology for its relevance to a society's need. This involves feedback loops and communication both ways and possibly going back to the drawing board for the TT professionals, adapting to conform to HTA requirements based on its horizon scanning and CTA, including HTA's Vol.

HTA is a way of assessing the ways science and technology are used in healthcare and disease prevention. It covers medical, social, economic legal, organizational and ethical issues. It provides policymakers with objective information, so they can formulate health policies that are safe, effective, patientfocused, context tailored and cost-effective. Five distinctive activities have been described by Sullivan et al. to define the HTA process [35]:

- Horizon scanning or early awareness alert systems;

- Topic determination and queuing;

- Collection and quality assessment of evidence;

- Appraisal;

- Assessment.

Early awareness and alert (e.g., horizon scanning) involves the early examination and active monitoring of emerging technology to determine, in part, potential evidence requirements, and budgetary implications $[32,33]$. CTA is a form of early HTA to minimize social conflicts, inadequate diffusion and unwise investments. CTA assesses the exact impact of a new technology to a broader approach, including the analysis of design, development and implementation of that new technology [36]. CTA can be done in parallel with the early awareness and alert system. 
Economic evaluation is a dimension of the HTA tool to determine the value of a new medical technology and is defined as 'the comparative analysis of alternative courses of action in terms of both their costs and their benefit' [37]. Decision-making between alternative courses of action in healthcare is based on evidence regarding clinical and cost-effectiveness and aims to maximize net health benefit. At the time of decision, clinical and cost-effectiveness evidence regarding the medical technology in question, may not be sufficient enough. This may result in a potentially wrong decision. Therefore, decision-makers would face the dilemma of taking an uncertain decision or expanding the evidence base to increase the net benefit of healthcare to society.

Vol analysis may answer this question by providing a framework for HTA, thus falling between questions 4 and 5 above. Vol estimates the potential benefits of gathering further information before making a decision [27]. Vol is a systematic decision-analytic approach to determine whether there is enough evidence to support new technologies, optimally designing research studies and setting research priorities for HTA [38]. Vol analysis helps decision-makers to allocate resources to further research regarding any chosen intervention in order to achieve the greatest return in terms of outcomes, for example, health gain from the resources available.

Using currently available information, it is possible to calculate the expected added value of the new technology against the existing practice, if any. Uncertainty in one or more parameters of current data (imperfect information) may adversely affect the precision of added value of the new technology. Thus, the uncertainty in the available data raises the possibility of a wrong decision. Vol analysis calculates the expected value of perfect information using methods such as the Bayesian method. The essence of the Bayesian approach is to provide a mathematical rule explaining how one should change one's existing beliefs in the light of new evidence. The difference between the expected value of the technology with current information and the expected value of the technology with perfect information is 'Vol'. This helps the decision-maker in research prioritization, as the cost of the new research for evidence base expansion should be lower than Vol. We believe that Vol could be used as a tool for research and research funding or investment prioritization and it has significant potential to assist in the decisionmaking process regarding the adoption of a technology into the practice [39].

It should be noted here that lobbying through advocating and reasoning, in a way that defends the conformity and relevance of the upcoming technology should be done by the $T T$ professionals 


\section{| CHAPTER II|}

(academic-industrial complex) and communicated with HTA in a polite manner before and/or throughout the process, or possibly at the end of a short initial assessment. This defense of conformity and relevance of the upcoming technology will be based on the Vol and PHG wheel reference frame questions answered, including HNA's assessment conformity. Of course it can be unavoidable that the TT professionals will have to go back to the drawing board to adapt to the HTA's assessment. Multiple contacts are made between the two parties. Early on involvement with all stakeholders is established here throughout the process and possibly earlier (10\% innovation network). These stakeholders, apart from the academic-industrial complex and HTA professionals, involve medical doctors, insurance companies and patient groups, among others. Feedback will be sought from these stakeholders to build a good case for the HTA assessment or post-assessment. Based on HTA's feedback and assessment, TT should adapt the emerging technology accordingly so that it conforms to the need as perceived by policy. This is how the proposal will be developed. This will complete $20 \%$ of the innovation network.

\section{Part 4: 40\% innovation network}

These potential interactions between TT and PHAT could possibly lead to fully fledged, broadly defined PPPs [17]. According to our original article [17], these PPPs are collaborative and involve bilateral communication, cross-talk and consultation, among others. The prelude to these fully fledged PPPs can be seen until $20 \%$ of the innovation network. The theory here is that, throughout this interaction, by the time it reaches $20 \%$ development of the innovation network, a sense of reliability and trust can develop and both parties will see the benefit of this PPP. For the academic-industrial complex, this will be from the perspective of saving resources and profit. The benefit of PPPs for policy-making can be seen as the realization of true horizon scanning [18] and timely interventions removing the issue of the circle of destination [18] (which is the cycle of late introduction of a then relevant technology, but now less relevant due to the functions of time and emerging technologies). Saving resources occurs not only from the perspective of time, but also from the fact that realizing early on that the technology development needs adaptations and, if not possible, can dictate whether or not to stop the project before its success or failure, respectively. Through these PPPs, the true sense of the impact of the technology can be investigated via HIA, as not only HIA data but company statistics are shared. HIA investigation starts in the TT pipeline from the early stages of intellectual policy rights through market introduction and its dynamics. For drug discovery, it starts at Phase IV. The HIA can significantly boost the case for TT towards the policy-makers by combining HTA recommendations, as well as the HNA. 
HIA, including post-introduction observation [40] is the process of identifying the future health consequences of a proposed action. It has been defined in the Gothenburg Consensus Paper that the HIA is a combination of procedures, methods and tools by which a policy, program or project may be judged by its potential effects on the health of a population, and the distribution of those effects within the population [41]. HIA is one way in which policy proposals, which affect determinants of health, can be assessed for both their potential intended and unintended consequences on the health of the population and the distribution of impacts within specific subgroups [42].

HIA includes the following elements:

Consideration of evidence about the anticipated relationships between a policy, program or project, and the health of a population;

- Consideration of the opinions, experience and expectations of those who may be affected by the proposed policy, program or project;

- Provision of more informed understanding by decision-makers and the public regarding the effects of the policy, program or project on health;

- Proposals for adjustments/options to maximize the positive and minimize the negative health impacts.

HIA offers a systematic five-step process for structuring actions aimed at identifying which health determinants will be affected by planning and conducting a study of the potential repercussions on a given population's health, and interacting with policy developers on the basis of the results.

The steps of HIA are as follows:

- Screening - to determine whether or not there exists the potential for significant health impacts as a result of a policy, program or project;

- Scoping - establishes the study area boundaries, identifies possible consequences, and determines a management approach for the HIA;

- Appraisal - considers the nature and magnitude of health impacts and the affected populations;

- Reporting - develops practical recommendations as to whether the decision-makers will consider the results of the HIA in their decisions;

- Monitoring - reviews the effectiveness of the HIA process and evaluates the actual health outcomes as a result of the project or policy. 


\section{| CHAPTER |||}

In addition to drawing on public health information produced through research, the HIA process is designed to accompany the process of public policy development, encouraging decision-makers and groups within the population to take part in identifying potential health effects. Therefore, HIA is an interaction between the public health sector, those responsible for the proposed policy and the population, whenever possible. This relates to our concept of PPPs.

\section{Part 5: 60\% innovation network}

Given that the above conditions are fulfilled through these now established PPPs, the LAL model will be reused in the development of other upcoming technologies. As a result, it is perceived that confident and streamlined processes will be developed over the second and third runs of the LAL model with the same policy agencies. Over several iterations or runs of the LAL model, the PPPs will recognize room for improvement in the innovation network in order to efficiently execute the model. As a result the innovation network matures enough to further shorten the time frame of this process. This is considered $60 \%$ coverage of the innovation network.

\section{Part 6: $80 \%$ innovation network}

Over time, the innovation network should develop into a system through repeated TT and PHAT interactions to involve decision-makers early on, along with the PHAT (of course these decision-makers heavily rely on PHAT). By this stage, it is perceived that the network of interactions will become stable and near perfected (not perfect, hence $20 \%$ always remains as room for improvement). So in a way, efficient assessments and adaptations have been implemented to bridge the gap between TT and PHAT and the timeframe has been standardized ending with HIA followed by the decision by policy-makers. This is the threshold we call the absorption capacity.

The steps are designed to show that, through the process of feedback loops, conformity requirements and reference frames, by the time the technology is ready for launch or launched, it can be readily taken up by healthcare systems in real-time as all assessments would have been met head on, which would have traditionally been taken after the technology information was in the public domain. In addition, the technology was adapted to the perceived requirements for policy guidelines. As a result, market penetration and reimbursement issues, among others, are resolved as guideline development is addressed by early dialog through the PPPs. In addition, timely intervention is a possibility, as is reducing 
the redundancy of a technology by its timely uptake $[17,18]$. A summary of our innovation network development and its steps are shown in Figure 3.

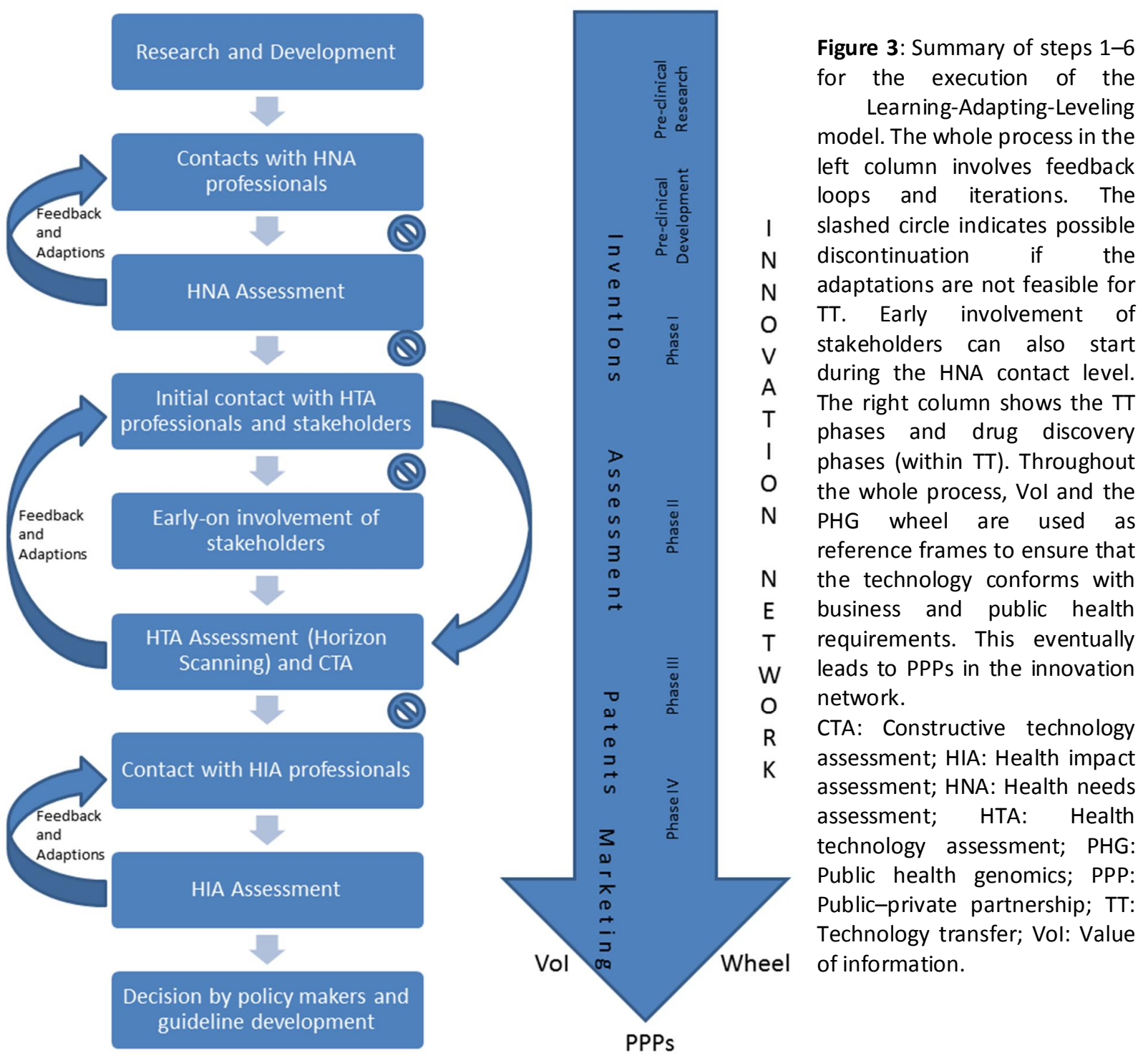


We aimed to develop concrete steps from the LAL model framework. Keeping the Vol and PHG wheel as the reference frame, we started off with defined steps initiated by research and development. After basic research and development, initial contact was made with HNA professionals by TT professionals or technology developers. After the HNA and subsequent feedback loops and adaptations, contact was made with HTA professionals and stakeholders. These stakeholders were given active involvement in the process, followed by HTA assessment in the form of CTA. After feedback loops and adaptations, contact was made with HIA professionals for the final round of assessments and feedback loops to be done by HIA. As a result the technology is then conformed to PHAT and ready to be forwarded to decision-makers for final decision and guideline development.

However, there are issues which need to be addressed. The Vol and PHG wheel reference frames are more of an indicative reference than a fully-fledged litmus test. Certain questions in the case of Vol (business) and steps in the case of Vol (HTA), as well as statements in the PHG wheel can be skipped based on the applicability of the technology or relevance of the reference frames' components to the technology's objective. This also applies to the stakeholder using it (TT for business Vol and TT/PHAT in the case of the PHG wheel). This is why piloting should be performed as it is required to demonstrate the applicability of the model itself and the modifications or tunings that may be required.

The steps involved for migrating the answering of the reference frames from negative to positive are not described as this will greatly vary from stakeholder to stakeholder depending upon their interest, capability and applicability, and are therefore of a subjective nature. This is outside the scope of the core steps themselves. The reference frames themselves are a prelude to the actual development of the innovation network.

HNA, HTA and HIA are sequential approaches with significant overlap in their aims, procedures and methodologies. All three deliver facilitating components for various phases of translational research, as described by Khoury et al. [43]. Furthermore, they go beyond these four phases. HNA, HTA and HIA have their own timeframes and independence from each other and TT. Also, a personalized healthcare intervention has been proposed with decision-making on a 'case by case' basis [44]. This will redefine the 
roles of HNA, HTA and HIA in translational research and its implementation. As a consequence, they do not currently compute with the LAL model, therefore an adapted version of each is necessary. This can also apply for TT to conform to the sequence of events. However, this still does not guarantee that PHAT professionals will be on board. As a result, it can be an absolute that $\Pi$ professionals will always have to contact PHAT to execute the LAL model. Furthermore, its tailored tuning to different technologies and their characteristics (which is also the proposal of Figure 1 ) will still have to be done. It can be possible, that over time, the model becomes a standard in some places, whereas elsewhere, it may not be that soon. In any case, the current economic landscape could encourage an enabler to a broader expansion of the model. Still, the model steps can be applied internally by $\Pi$ professionals, including the PHAT assessments, the reason for which is elaborated on in the text. The distinct advantage of doing so is that, even if PHAT are not on board, $\mathrm{TT}$ is still able to conform their technologies to healthcare and public health requirements, possibly reaping a similar benefit. This should not be considered a replacement of contact, as networking in the innovation network is required for real-time acceptance into healthcare and the development of PPP can be beneficial in the long term. The non-synergy between TT and PHAT is the inherent problem, and to resolve this, their bilateral communication is required. Therefore, it is not recommended to just stick to internal assessments.

In relation to the above paragraph, timelines of assessments in the LAL model (Figure 3) cannot be defined at this stage. The timeframe we suggest is ideal (5-10 years; this is quicker than normal, but can perhaps be still too long for the integration of a new molecular diagnostic assay), with HNA, HTA and HIA being 1,3 and 2 years, respectively (Figure 2), for the 5 -year period. However, when previous work has been performed, especially in the case of HNA, it can be shortened. On the other hand, however, to address this issue, current systems in place have evolved over time to a more structured, transparent and informative way of execution. Nonetheless, currently existing technologies are required in many senses to enable and optimize this structuring and potential restructuring possibly through the LAL model. This also includes feedback loops (Figure 1). However, even if we were able to adapt HNA, HTA and HIA within the timeframes, adaptations on the feedback would require additional time. Initially, when the model is applied, time can increase due to the added steps outside of $\Pi$ 's domain. Nonetheless, it is advantageous compared with the time lost from market to healthcare systems (circle of destination) [18]. In the long run, the timeframe can be reduced and eventually adapted to be sustainable for the PPPs. These PPPs can take more time to come into form and a few iterations may be required before the PPPs are formalized. However, according to our broadly defined PPPs, this also 


\section{| CHAPTER II|}

involves consultation and two-way communication. The decision-makers in Figure 1 are indirectly present through their domains of HNA, HTA and HIA (these give recommendations to decision-makers). Here, over time, decision-makers can also be more directly involved through these PPPs in the innovation network. Lobbying can bring ethical, legal, societal and organizational issues of misled or hidden agendas, which we proposed in our original article [17]. As well as their own source, HTA should have, apart from their own source and the TT data, a third opinion, for example, the patient groups when these PPPs are formalized.

The quantification of TT products to a single qualitative product (see Figure 1 inverse triangle) [17], does not hinder its process when getting feedback from PHAT, as through feedback, options can be added or reduced, eventually choosing one with which to go forward. Of course, this does not imply that TT should show several options to PHAT as they will outright reject it, unless some of them are worth investigating (in their opinion). TT can identify which of their products through the reference frames are worth forwarding to PHAT for investigation, or if the current one needs to be replaced with a new one halfway through, possibly bringing it back to square one.

Irrespective of the issues stated above, the problem of this non-synergy and inexistence of crossings are the main issues $[17,18]$ and the LAL model, through its steps, ensures all aspects of business and public health, as well as healthcare issues, are covered [18]. This non-communication causes a delay in the uptake of relevant GBITs in healthcare, therefore bilateral communication is necessary to ensure realtime interventions. In other words, as we elaborated, the LAL model covers all possible areas of business, public health and healthcare requirements which can be neglected. Being the overarching framework [18] through its extensive coverage of these neglected areas, the LAL model should be implemented. Other issues in the delay to healthcare integration can include lack of funding to research institutes and limited return on investments for some diagnostics as compared with pharmaceuticals and biologics. However, for the limited return on investments, this can also be an issue of not implementing the LAL model, as conformity issues being resolved can open currently inaccessible markets and/or healthcare systems more quickly for better returns in real-time. Coming back to lack of funding, it can be seen as another issue, in itself detached from the major issue of cross-cutting communication. Even if the funding issue is resolved, communication barriers, which we have elaborated on in this article, will still remain, which can be resolved through the LAL model. Apart from this, clinical utility of current GBITs limit their usage in healthcare systems. However, this again is an issue related to the LAL model as the 
oversight of the clinical utility is a product of this lack of stakeholder engagement between healthcare systems and industry, which our model resolves from the perspective of conformity. A game theory approach to these situations as well as the LAL model interactions in general can be an interesting endeavor. 
The documented backlog of relevant GBITs inhibits the deployment of personalized medicine applications in healthcare. This is evident by the promises of the post-genomic era through systems biology regarding a truly personalized treatment, compared with its actual execution in healthcare systems. We believe that the LAL model, through its steps, can resolve this issue of backlog of relevant GBITs, and thereby release the GBITs in an effective and efficient manner in real-time for personalized applications in healthcare.

We have described the LAL model step-by-step as to how it should be used. The Vol and PHG wheel should go throughout the LAL model processes of execution to ensure that all business, as well public health, issues are covered. Iterations are important as adaptations and conformity issues are resolved through the process, ensuring that by the time the technology is rolled out, it conforms to decisionmakers for real-time uptake and policy guidelines. TT starts slightly earlier than HNA and, later on, moves in parallel with HNA, HTA and HIA in the same order as assessment feedback. A case is built for the decision-makers of the technology being developed through feedback, reference frames and approval from each domain (HNA, HTA and HIA). The innovation network develops accordingly, with early involvement of all stakeholders. PPPs are developed within the innovation network over some iterations.

Timeline issues of the steps involved still need to be defined as this may vary from type of technology to assessment timeframe, as well as TT and PHAT tools adaptability to each other. Over time, however, this can be reduced and become sustainable. During this time, internal assessment of PHAT by $\Pi$ can be performed; however, this is not recommended in the long term, as it will not resolve the issue of nonsynergy, possible stagnating profits. Through these steps, real-time uptake of GBITs by healthcare in an effective and efficient manner becomes plausible. Apart from decreasing the timeframe to healthcare in the long run, not only can $\pi$ come to an early decision to continue or kill the technology through PHAT feedback, thus saving on resources, but it can also tap into a previously unexplored market due to timeframe constraints. In other words, although businesses make profits on who they sell their technologies to, if the whole healthcare system accepts their product, that is a larger market than traditionally sold to for example, a few hospitals, research centers or other companies, among others. In time, however, there may be a chance that eventually the healthcare systems accept (or reject) the 
technology, which gives more reason to execute the LAL model to ensure acceptance. Also, healthcare systems can benefit from timely interventions and technologies reaching the patient in an effective and efficient manner. As a result, this could positively impact on health outcomes. Previous ways of doing this, although they, in some cases, provided benefits to manufacturers and systems, are not possible under the current economic landscape and, thus, this approach is more likely to be successful because it provides a rational, structured and logical process to introduce added value technologies in healthcare systems. The described methodology of the LAL model framework can benefit all stakeholders who use it. Although the model is being implemented in some contexts as this article goes into publication, its proof of concept still remains to be seen at the end of the technology maturation process. Nonetheless, the LAL model framework, given its overarching frame [18], can be the answer to cross-integration [19] and a focus on GBITs might eventually make it a possible tool for the implementation of personalized medicine.

\section{Future Prospects}

We believe that if the model is properly lobbied, although it is currently being advocated including through grants, high level policy reports and industry support, it can become the de facto model for the evaluation of GBITs for effective and efficient integration of personalized medicine applications in healthcare in a timely manner. As our current work is inclined to pilot studies to test the robustness of the LAL model, we aim to tweak and refine TT, HNA, HTA and HIA to each other for streamline a performance. Over time, this will be achieved and the general acceptance will grow as we work closely with several agencies and industries. Furthermore, elsewhere we have demonstrated why the LAL model is an overarching framework covering all aspects of healthcare integration, building its case. In addition, we see growing evidence of demand for early dialog and early involvement of all stakeholders with programs and initiatives towards this end. The LAL model addresses this early dialog and stakeholder involvement. Given the fact that the framework constitutes all of the necessary tools and early dialog demanded for healthcare integration, it may eventually be used in different adapted forms by relevant stakeholders. As a result, it may be an essential tool used in personalized medicine for healthcare implementation. 


\section{| CHAPTER |II}

\section{Executive Summary}

\section{Delayed personalized medicine application}

- Personalized medicine has become the central focus of the concept 'one shoe does not fit all', resulting in a more personalized approach towards healthcare.

- However, the implementation of personalized medicine applications such as genome-based information and technologies is not completely seen.

\section{Bottleneck and solution}

- This bottleneck is due to the fact that there is lack of communication between the academicindustrial complex and healthcare assessment professionals.

- We expand upon the innovative Learning-Adapting-Leveling model framework as this framework addresses the bottleneck.

\section{Developed concrete steps}

- We develop in chronological order the steps of this model to resolve the issue of delayed implementation

- The value of information from the business perspective and health technology assessment perspective as well as the ten essential public health tasks are used as reference frames for early detection of issues throughout the process.

- Throughout the technology transfer activity, the academic-industrial complex makes contacts with the health needs assessment, health technology assessment and health impact assessment professionals in the same order.

- These contact lead to assessments, adaptations and feedback loops.

- As a consequence, early involvement of all stakeholders is guaranteed.

\section{$\underline{\text { Key issues }}$}

- This model addresses the effective and efficient implementation of personalized medicine applications to healthcare in a timely manner.

- However, the model still needs to be tweaked for compatibility between technology transfer, health needs assessment, health technology assessment and health impact assessment, including timelines of parallel work.

- We believe the model can become the overarching framework in personalized medicine for healthcare integration of genome-based information and technologies. 


\section{References}

1. Alon U, Surette MG, Barkai N, Leibler S: Robustness in bacterial chemotaxis. Nature 1999, 397:168-171.

2. Goh KI, Cusick ME, Valle D, Childs B, Vidal M, Barabasi AL: The human disease network. Proceedings of the National Academy of Sciences of the United States of America 2007, 104:8685-8690.

3. Jordan JD, Landau EM, lyengar R: Signaling networks: the origins of cellular multitasking. Cell 2000, 103:193-200.

4. Levine $\mathrm{K}$, Tinkelenberg AH, Cross F: The CLN gene family: central regulators of cell cycle Start in budding yeast. Progress in cell cycle research 1995, 1:101-114.

5. Little JW, Shepley DP, Wert DW: Robustness of a gene regulatory circuit. The EMBO journal $1999,18: 4299-4307$.

6. Weng G, Bhalla US, Iyengar R: Complexity in biological signaling systems. Science 1999, 284:92-96.

7. Yi TM, Huang Y, Simon MI, Doyle J: Robust perfect adaptation in bacterial chemotaxis through integral feedback control. Proceedings of the National Academy of Sciences of the United States of America 2000, 97:4649-4653.

8. Edelman GM, Gally JA: Degeneracy and complexity in biological systems. Proceedings of the National Academy of Sciences of the United States of America 2001, 98:13763-13768.

9. Schernhammer ES, Laden F, Speizer FE, Willett WC, Hunter DJ, Kawachi I, Colditz GA: Rotating night shifts and risk of breast cancer in women participating in the nurses' health study. Journal of the National Cancer Institute 2001, 93:1563-1568.

10. Mancinelli L, Cronin M, Sadee W: Pharmacogenomics: the promise of personalized medicine. AAPS pharmSci 2000, 2:E4.

11. Sanchez C, Lachaize C, Janody F, Bellon B, Roder L, Euzenat J, Rechenmann F, Jacq B: Grasping at molecular interactions and genetic networks in Drosophila melanogaster using FlyNets, an Internet database. Nucleic acids research 1999, 27:89-94.

12. Rappaport SM, Smith MT: Environment and Disease Risks. Science 2010, 330:460-461.

13. Kitano H: Computational systems biology. Nature 2002, 420:206-210. 


\section{CHAPTER III}

14. Lal JA, Brand A: Functional dynamics: from biological complexity to translation and impact in healthcare systems. J Comput Sci Syst Biol 6: 088-092.

15. Sorensen K, Brand H: Health literacy: the essential catalyst for the responsible and effective translation of genome-based information for the benefit of population health. Public health genomics 2011, 14:195-200.

16. Cesuroglu T, van Ommen B, Malats N, Sudbrak R, Lehrach $H$, Brand A: Public health perspective: from personalized medicine to personal health. Personalized Medicine 2012, 9:115-119.

17. Lal JA, Schulte In den Baumen T, Morre SA, Brand A: Public health and valorization of genome-based technologies: a new model. Journal of translational medicine 2011, 9:207.

18. Lal JA, Morre SA, Brand A: The overarching framework of translational and integration: a case for the LAL model. Personalized Medicine 2013, In Press.

19. Syurina EV, TSidB, Brand A, Ambrosino E, Feron FJM: Concepts for the translation of genomebased innovations into public health: a comprehensive overview. Personalized medicine 2013, 10:163-167.

20. Crow MM, Tucker C: The American research university system as America's de facto technology policy. Science and Public Policy 2001, 28:2-10.

21. Mullins B CJ: Technology transfer: A roadmap. In Book Technology transfer: A roadmap. 1999.

22. Rosenkotter N, Vondeling H, Blancquaert I, Mekel OC, Kristensen FB, Brand A: The contribution of health technology assessment, health needs assessment, and health impact assessment to the assessment and translation of technologies in the field of public health genomics. Public health genomics 2011, 14:43-52.

23. Beskow LM, Khoury MJ, Baker TG, Thrasher JF: The Integration of Genomics into Public Health Research, Policy and Practice in the United States. Community genetics 2001, 4:2-11.

24. Brand A, Lal JA: European Best Practice Guidelines for Quality Assurance, Provision and Use of Genome-based Information and Technologies: the 2012 Declaration of Rome. Drug metabolism and drug interactions 2012, 27:177-182.

25. Lal JA, Malogajski J, Verweij SP, de Boer P, Ambrosino E, Brand A, Ouburg S, Morre SA: Chlamydia trachomatis infections and subfertility: opportunities to translate host pathogen genomic data into public health. Public health genomics 2013, 16:50-61. 
26. Verhoef PC, Lemon KN: Successful customer value management: Key lessons and emerging trends. European Management Journal 2013, 31:1-15.

27. Claxton K, Neumann PJ, Araki S, Weinstein MC: Bayesian value-of-information analysis. An application to a policy model of Alzheimer's disease. International journal of technology assessment in health care 2001, 17:38-55.

28. Stewart JJ, Allison PN, Johnson RS: Putting a price on biotechnology. Nature biotechnology 2001, 19:813-817.

29. Douw K, Vondeling H, Eskildsen D, Simpson S: Use of the Internet in scanning the horizon for new and emerging health technologies: a survey of agencies involved in horizon scanning. Journal of medical Internet research 2003, 5:e6.

30. Brown IT, Smale A, Verma A, Momandwall S: Medical technology horizon scanning. Australasian physical \& engineering sciences in medicine / supported by the Australasian College of Physical Scientists in Medicine and the Australasian Association of Physical Sciences in Medicine 2005, 28:200-203.

31. Stevens A, Gabbay J: Needs assessment needs assessment. Health trends 1991, 23:20-23.

32. Gutierrez-Ibarluzea I, Simpson S, Benguria-Arrate G: Early awareness and alert systems: an overview of EuroScan methods. International journal of technology assessment in health care 2012, 28:301-307.

33. Packer C, Gutierrez-Ibarluzea I, Simpson S: The evolution of early awareness and alert methods and systems. International journal of technology assessment in health care 2012, 28:199-200.

34. Lampe K, Mäkelä M, Garrido MV, Anttila H, Autti-Rämö I, Hicks NJ, Hofmann B, Koivisto J, Kunz R, Kärki P, et al: The HTA Core Model: A novel method for producing and reporting health technology assessments. International journal of technology assessment in health care 2009, 25:9-20.

35. Sullivan SD, Watkins J, Sweet B, Ramsey SD: Health technology assessment in health-care decisions in the United States. Value in health : the journal of the International Society for Pharmacoeconomics and Outcomes Research 2009, 12 Suppl 2:S39-44.

36. Douma KF, Karsenberg K, Hummel MJ, Bueno-de-Mesquita JM, van Harten WH: Methodology of constructive technology assessment in health care. International journal of technology assessment in health care 2007, 23:162-168. 


\section{| CHAPTER III}

37. Drummond MF, Sculpher MJ, Torrance GW: Methods for the economic evaluation of health care programmes. Oxford University Press; 2005.

38. Eckermann S, Karnon J, Willan AR: The value of value of information: best informing research design and prioritization using current methods. PharmacoEconomics 2010, 28:699-709.

39. Steuten L, van de Wetering G, Groothuis-Oudshoorn K, Retel V: A systematic and critical review of the evolving methods and applications of value of information in academia and practice. PharmacoEconomics 2013, 31:25-48.

40. Varela-Lema L, Ruano-Ravina A, Mota TC, Ibargoyen-Roteta N, Imaz I, Gutierrez-Ibarluzea I, Blasco-Amaro JA, Soto-Pedre E, Sampietro-Colom L: Post-introduction observation of healthcare technologies after coverage: the Spanish proposal. International journal of technology assessment in health care 2012, 28:285-293.

41. Policy WECfH: Health impact assessment: main concepts and suggested approach. The Gothenburg Consensus Paper, WHO Regional Office for Europe, ed. 1999, Brussels:World Health Organisation. In Book Health impact assessment: main concepts and suggested approach. The Gothenburg Consensus Paper, WHO Regional Office for Europe, ed. 1999, Brussels:World Health Organisation.

42. Mindell J, Bowen C, Herriot N, Findlay G, Atkinson S: Institutionalizing health impact assessment in London as a public health tool for increasing synergy between policies in other areas. Public health 2010, 124:107-114.

43. Khoury MJ, Gwinn M, Yoon PW, Dowling N, Moore CA, Bradley L: The continuum of translation research in genomic medicine: how can we accelerate the appropriate integration of human genome discoveries into health care and disease prevention? Genetics in medicine : official journal of the American College of Medical Genetics 2007, 9:665-674.

44. Gutiérrez-Ibarluzea I: Personalised Health Care, the need for reassessment. A HTA perspective far beyond cost-effectiveness Italian Journal of Public Health 2012, 9.

\section{Internet References}

[101] Executive Agency for Health and Consumers. Call 2013: tenders.

http://ec.europa.eu/eahc/health/tenders H09 2013.html

[102] IT Future of Medicine. www.itfom.eu

[103] Public Health Genomics. www.phgen.eu

[104] Green Park Collaborative. www.greenparkcollaborative.org 
[105] Measuring and valuing customer relationships. How to develop the measures that drive profitable CRM strategies. www.business-intelligence.co.uk/reports/measure $\mathrm{crm} /$ summary.asp

[106] The University of Birmingham (UK). An introduction to HCNA.

www.birmingham.ac.uk/research/activity/mds/projects/HaPS/PHEB/HCNA/intro/index.aspx

[107] Division of Research and Economic Development. Technology transfer.

http://cmsserv.ncat.edu/doclib/DORED,\%200utreach\%20and\%20Technology\%20Transfer\%20(How\%20T o\%20Section)\%20Web\%20site,-5.pdf

[108] PKD Foundation. Drug development timeline. www.pkdcure.org/research/drug-

development/timeline

\section{Acknowledgements}

This project is supported by a grant from the European Commission PHGEN II (Duration period: June, 2009-November 2012 EU-Project No. 20081302). This work is also supported by the Canadian Institutes for Health Research (CIHR), CIHR Institute of Genetics, the CIHR Institute of Health Services and Policy Research through a grant (no. ETG92250) to the APOGEE-Net/CanGèneTest Research and Knowledge Network on Genetic Services and Policy. We also like to extend our thanks to the research school GROW of the Faculty of Health, Medicine and Life Sciences of Maastricht University for supporting this research. 

CHAPTER IV

CONCLUSIONS PART A 


\section{Conclusions}

With regard to specific objectives of Part $A$, with respect to chapter I, the thesis completed and answered the objectives pertaining namely:

- Presenting the problem statement of the bottleneck of integration of genome-based technologies into healthcare

Based on the exponential increase in genome-based technologies (including personalized healthcare applications) onto the market, patents and scientific literature, there seems to be a backlog of integration of these technologies into healthcare. Although there are tools and systems in place for move from bench to healthcare, still the backlog remains regarding the real-time effective and efficient real-time integration of genome-based technologies. The chapter identified the reason for the bottleneck mainly being the non-synergy or lack of communication between the academic-industrial complex and the decision makers of healthcare integration. Furthermore, these two entities use different tools for translational research

- Identifying the rationale behind the problem statement

The rationale behind the problem of the realtime effective and efficient integration of genome-based technologies is the different tools used by the non-synergistic academic-industrial complex and the decision makers for healthcare

\section{Take Home Message}

Although there is an exponential growth of genome-based technologies its real time effective and efficient integration in healthcare is not realized.

The bottleneck is identified as the non-synergy between the academic-industrial complex and health policy decision making.

These two entities namely use different tools for translation (Technology Transfer) and implementation (Public Health Assessment Tools) respectively used in different timeframes.

By the time a new technology enters healthcare it becomes outdated as a newer technology presents itself on the market.

The Learning-Adapting-Leveling model resolves this bottleneck by bringing Technology Transfer and the Public Health Assessment Tools together for the first time on a common platform in parallel along with other concepts.

The protocol developed on this framework can help industry to come to an early on decision saving on resource as well as possibly help decision maker diffuse relevant technologies and develop policies.

The model has been argued as an overarching framework and has been demonstrated accordingly. Conceptual issues of tools used have been cleared. Issues regarding compatibility of the tools in the model still need to be resolved. 
integration. As a consequence, by the time a technology reaches the healthcare, it can be considered less relevant compared to a new emerging technology on the market. This is a continued cycle. As a result, both industry and healthcare systems including individuals (both patients and healthy people) are at a disadvantage. For the industry, they are unable to tap into a much larger market than currently perceived. For example the industry sells their technology or product to a few research hospitals, labs and partners making millions in the process. However, if healthcare accepts the technology and its widespread usage becomes a reality, it becomes a much larger market, generating larger sums of profit. Furthermore, several technologies perceived by the academic-industrial complex are answering the need generally get rejected or delayed by the healthcare. If the academic-industrial complex can come to an early-on decision to the developing and emerging technology regarding its acceptance and conformity to healthcare requirements, it can save a lot on resources like time and money. In addition the academic-industrial complex based on the new information, make the decision to either adapt the technology before market introduction or kill it entirely or continue with it for the traditional perceived market. On the other hand, both citizens and healthcare are at a disadvantage due to the delay of relevant genome-based technologies. Reason being this relevantly blocked technology may be more effective and efficient than the currently utilized one. As a possible consequence, for example the emerging diagnostic technology can predict susceptibility or onset to a disease better than the currently utilized one, if integrated timely. It can possibly reduce the burden of disease through the concept of early prevention better than only by cure. Similarly, patients can benefit from other genome-based technologies if integrated on time.

- Introducing tools to resolve the problem statement In this chapter it was concluded that technology transfer is the most widely used tool for commercialization of technologies from bench (the lab) to the market and return on investments. This is mainly used by the academic-industrial complex for valorization and commercialization. On the other hand, HNA, HTA and HIA are the most widely used tools referred to by decision makers to come to a decision on healthcare integration and allocation of resources. Furthermore the value of information is considered pivotal for 
decision making. Also the PHG wheel seems relevant for its inclusion as a good reference point for integration into public health.

- Defining valorization in public health genomics

Valorization in public health genomics is defined as the process of realization of added value bioproducts in the domain of public health for benefit of the population and healthcare systems. Here realization is considered in terms of understanding the importance, impact or potential benefit and implementing the bioproduct. Bioproducts are defined as sustainable novel products or products satisfying novel health applications derived or inspired from genome-based information or technologies. Genome-based means everything deriving from the human genome, for example all 'omics', like genomics, proteomics, metabolomics, etc. Genome-based information implies 'omics' interactions with environmental factors like lifestyle, location, climate, etc. Technologies can be a technique, process, clinical application, diagnostic kit, methodology, activity or technology in the general sense.

- Developing the framework to close the gap - the LAL model

The Learning-Adapting-Leveling model was developed using the methodology of an integrated approach of the basic design cycle and the fish trap model. Based on the reasoning in the methodology, tools were selected or discarded for their relevance to the problem. The Learning-Adapting-Leveling model for the first time brings together the two major activities used by the academic-industrial complex and the decision makers, namely technology transfer and the public health assessment tools (HNA, HTA, HIA) respectively in a pseudo-parallel initiation. The proposal is, that through this parallel initiation, there will be interactions and feedback loops to conform the technology to the healthcare requirements. These interactions will be on a platform defined as the innovation network, possibly leading to broadly defined public-private partnerships. During the whole process, the model takes into consideration the value of information and the public health genomics wheel as reference points to ensure that all business aspects and as well as healthcare aspects are addressed by the time the technology is rolled out into the market. As a result, it can be readily taken up by healthcare system as 
the technology would now conform to its requirements. This over time will be optimized with an operational threshold which is defined as the absorption capacity.

With regard to specific objectives of Part A, with respect to chapter II, the thesis completed and answered the objectives pertaining namely:

- Elaborating on various concepts used in the field and arguing the exclusion/inclusion of them with respect to the developed framework

In this chapter, all relevant concepts were described and defined which are used for translational research. The tools or activities defined and described were valorization, spin-offs, regional valorization systems, open innovations, drug discovery, HNA, HTA, HIA, ACCE, EGAPP, T1-T4, PHG Enterprise, and PHG Wheel. Based on the definition of technology transfer, and in parallel to definitions of valorization, regional valorization systems, spin-offs, open innovations and drug discovery were placed within the overarching framework of technology transfer. Technology transfer was described to be the most popular tool or activity used for commercialization of technologies onto the market and return on investments so it was considered one of the two core components of the Learning-Adapting-Leveling model. Similarly, HNA, HTA and HIA in the form of the public health assessment tools are widely used assessment tools referred to by decision makers in healthcare systems with defined methodology. Therefore it was the other core component of the Learning-Adapting-Leveling model. On the other hand ACCE, EGAPP, T1-T4 and the PHG Enterprise although having valid points in their own application were left out due to their assorted weaknesses or ill-defined methodologies and/or overlaps with existing tools in the Learning-Adapting-Leveling model like the PHG wheel. The PHG wheel was demonstrated to show its overarching framework over the rejected tools and overlaps to HNA, HTA and HIA, therefore was considered an important part of the model. Reason for it being not considered at par with HNA, HTA and HIA was that unlike them the PHG wheel does not have a defined methodology rather just mentions the 10 essential public health tasks, which address public health integration and the way of doing it (e.g. using HTA). Nonetheless, the PHG wheel is a good reference point to ensure that aspects of even rejected tools are covered and possible overseen gaps can be addressed. 
- Resolving the issue of overlapping tools and defining them with relation to each other.

Through the definitions in this chapter, the tools with a commercialization aspect seem to greatly overlap with each other. It was cleared that translational research covers the whole aspect from bench (lab) through the market to healthcare. For the first half, i.e. from lab to market, technology transfer is considered the overarching framework. Technology transfer was defined to demonstrate its encompassing various tools or concepts (valorization, regional valorization system, open innovation and drug discovery and spin-offs) within commercialization and defined their slots in time through the process.

- Argumentation why the solution framework is the answer

The Learning-Adapting-Leveling model brings for the first time the two widely used and popular activities for translational research namely, technology transfer and the public health assessment tools (HNA, HTA and HIA) onto a common platform (innovation network) and aims for broadly defined public-private partnerships through these interactions. Technology transfer addresses the move from lab onto the market and the public health assessment tools define the move from the market to healthcare integration through recommendation given to decision makers. This chapter defined the comprehensibility of the PHG wheel (part of the model) as an overarching reference frame to ensure that all aspects are covered including rejected tools. Similarly the value of information ensures that the relevance, processing ability in terms of understandability of the technology to the end user and exclusivity in terms of patents which restrict the wide usage are addressed. The value of information is dealt from both a business aspect and HTA aspect for early on assessment. In addition, the model encourages early-on involvement of all stakeholders. The model is quite comprehensive in its reach of issues to be addressed from both a business as well as from the healthcare perspectives due to its extensively defined tools. Also the model has been heavily cited, presented, published in high level reports and is being implemented in pilot studies. Moreover, the model has been developed specifically for the bottleneck defined. Therefore, it should be considered an overarching framework and the solution to the defined bottleneck. 
With regard to specific objectives of Part A, with respect to chapter III, the thesis completed and answered the objectives pertaining namely:

- Elaboration on the defined steps of the solution framework

The defined steps start off with basic research and development in the lab. After some initial feasibility tests and the development of the idea which would answer the need or create one, the technology transfer professionals make contract with the HNA professionals. The HNA professional do an initial assessment and this leads the technology transfer professionals back to the drawing board and adapt. Thereafter, the technology professionals contact the HTA professionals with their conformed technology, keeping in mind the HTA's value of information. The HTA professionals assess the technology and give feedback based on that assessment. The technology professionals go back to the lab and conform the technology further. The same step is repeated with the HIA professionals. This can involve several feedback loops per agency. Also the PHG wheel and the value of information are taken as a reference point throughout the process to ensure that all aspects are addressed. As a consequence, by the time the technology is rolled out onto the market, through relevant lobbying based on the conformity issues addressed, the technology can be readily taken up by decision makers who assess it based on the original assessment requirements addressed. The aim is that overtime of these interactions public-private partnerships will be developed and will be optimized.

- Addressing issues still to be resolved Initially through this process, the timeframe will be increases as additional steps will be added in the technology transfer pipeline, however in the long run this will be optimized and compared to the delayed uptake, it is a good tradeoff. Also HNA, HTA and HIA have to be compatible to each other as well as technology transfer which are believed to be resolved through the development of public-private partnerships. Furthermore, not all aspects of value of information and the public health genomics wheel need to be addressed depending on the type of the technology or the user of the Learning-AdaptingLeveling model. However, addressing these reference points from negative to positive will enhance the possibility of healthcare integration. Another point to be noted is that how 
to bring these reference points from negative to positive is not answered here as this is entirely subjective depending upon the user of the reference points. Even after the model's wide attention, it does not guarantee that it will be widely used. As an initial solution the technology transfer professionals should do these assessments internally however this should not replace the actual networking, which is the problem to begin with. The internal assessment will only prepare for perceived assessments in a noncooperative manner. Another point is that ethical issues can develop as a result of lobbying which should be addressed using the public health assessment tools recommendations, the technology transfer proposal and a third opinion from patient groups and external ethical committees. 
PART B

POTENTIAL IMPLEMENTATION OF LAL MODEL

IN PRACTICE 


\section{Specific Aims}

After the development of the Learning-Adapting-Leveling model as the theoretical solution framework to the bottleneck of effective and efficient real-time integration of genome-based technologies and laying the foundation of theoretical concepts and argumentation of the model as the overarching framework this section of the thesis aims to document potential scenarios where the model could be implemented given its protocol has been defined. The use of the model and its potential scenarios has already been mentioned in various relevant national as well as European policy reports and other key documents. Also this part aims to contribute to the development of European best practice guidelines for genome-based knowledge and technologies including personalized healthcare applications as current guidelines do not compute with emerging genome-based information and technologies for personalized medicine applications. The aims of Part B relate to the following general objectives mentioned in the introduction chapter:

- Giving examples where the new framework can be implemented

- Contribution to the development of the European best practice guidelines on genome-based information and technologies

The specific aims of this section derived from the general objectives above are as follows:

- Chapter V

- Demonstrate the complexity of biology reasoning for the move in the post-genomics era to systems biology and systems medicine for personalized health applications and beyond

- Signifying the issue of taking into account environmental factors interacting with 'omics' factors as well as health systems when developing such applications

- Using the EU flagship pilot project ICT Future of Medicine (ITFoM) as example of European research project where the Learning-Adapting-Leveling model is being implemented

- Chapter VI

- Applying it to Chlamydia trachomatis as the leading cause in tubal pathology and infertility

- Demonstrating the implementation of the Learning-Adapting-Leveling model to potential SMES

- Chapter VII

- Contributing to the development of the European best practice guidelines for genomebased information and technologies (PHGEN II)

- Integration of the Learning-Adapting-Leveling model into these guidelines

- Chapter VIII

- Conclusions based on the specific aims mentioned 
CHAPTER V

FUNCTIONAL DYNAMICS: FROM BIOLOGICAL COMPLEXITY TO TRANSLATION AND IMPACT IN HEALTHCARE SYSTEMS

Published as:

Lal JA, Sudbrak R, Lehrach $H, B$ Band A.

Journal of Computer Science and Systems Biology 2013, 6(2):88-92. 
Biological complexity at a molecular and physiological level is dynamically translucent and requires a system-wide computational approach to possibly elucidate underlying mechanisms for medical and public health applications. Functional dynamics is ideal to study molecular functions given biological functions are dependent on the dynamic nature of networks it operates within. However, environmental factors significantly affect the molecular dynamics in biology, which still needs to be incorporated in study of functions for medical applicability. Through technological innovation medicine is seeing a potential shift in demand for personalized interventions, which has not been fully realized yet. Also the applicability of functional dynamics' utility seems not visible in healthcare systems. This article addresses the above mentioned issues, challenges in translation/implementation using the example of the "virtual patient" developed through the pilot EU flagship project ICT Future of Medicine, and provides possible solutions and insights of new and existing scientific data, infrastructures and frameworks like the Learning-Adapting-Leveling model to make it feasible including policy-wise by incorporating best practice guidelines developed through the Public Health Genomics European Network and tries to touch upon its consequential impact. As a result, we see that real time integration in healthcare requires early-on involvement of all stakeholders as well as taking into account health policy issues, which is addressed by the proposed Learning-Adapting-Leveling model and the best practice guidelines. Furthermore, environmental factors and exposome properties need to be taken into consideration, which the pilot ICT Future of Medicine has been taken into account. We now possibly see a shift from stratified medicine through personalized medicine and possibly towards individualized medicine. This coupling of the pilot project ICT Future of Medicine by integrating the Learning-Adapting-Leveling model to resolve real-time integration issues and considering policy-wise the best practice guidelines has set the stage for it to potentially revolutionize the healthcare system as a whole.

Keywords: Computational biology, Functional dynamics, Information and communication technologies, Public health genomics, Personalized medicine, Systems biology, Genomics, Health technology assessment, Health needs assessment, Health impact assessment, Technology transfer. 


\section{The Complexity of Biology}

Over the progression of scientific knowledge and discovery especially in the field of genome-based information and technologies (encompassing all-omics, for example genomics, transcriptomics, proteomics, metabolomics, etc. and their interaction with environmental factors), we come short of understanding the complexity of the highly dynamic molecular human biology with all permutations and combinations of interactions and physiology itself; rather we are only able to grasp the complexity of it. What makes the molecule level biology far complex is that not only does function rely on the molecule (genes, proteins, transcription factors, metabolites, etc.) itself, but also relies with the combination of network of interactions (for example gene-gene, gene-protein, protein-protein, 1 protein-12 proteins, etc.) it is attached. This can constitute feed-back/forward control [1,2], modularity [3], redundancy [4] and multitasking [5]. The function of operation can also be dependent on the structure of the molecule and/ or the structural stability of the network [6] and localization. Certain molecules can act as genetic switches among others. Inhibiting a molecule (say gene) may not inhibit the function itself, but being linked to a stable network and/or complimentary genes can compensate. Also networks can be unaffected to perturbations as complimentary yet unrelated networks can stand in. In addition to that the combination of the functional molecule and the network itself is required for execution of the function as separated, may not work. Furthermore, large well connected networks are least affected to perturbations compared to not so well connected networks, however the prior can be fragile if the most influential nodes are corrupted. This is all being in a highly dynamic, constantly adapting and changing environment and at different levels (molecular, cellular and tissues). A very nice review to this end has been written by Kitano already in 2002 [7]. What adds to the complexity of the network is that previously unconnected diseases or genes seem related and have not yet been medically classified accordingly [8].

\section{Systems Approach}

From the above, what can be understood is that although single gene/protein research is still required to gather information about function and processes, a more holistic approach is needed to elucidate the functional complexity of a system, which is the human. This also implies for drug discovery and targeting as well as therapy. Through -omics based research, we now know that functionality or execution of a process is dependent upon multiple molecules and networks and no one molecule or network can work 


\section{CHAPTER V}

on its own, therefore can be viewed as a system. Drug targeting now requires consequences to the network (or so called side-affects). The data generated by this information, especially in biological systems impracticably large, consequentially make it dependent on computational power to store, analyze and interpret it unlike what was previously thought to be localized to the wet lab. This has been evident from the very basic of biological computing in BLAST [9] to data analysis [10,11], among others [12-14]. This convergence of biology and computation power has been recently discussed [15], stating the fact that the two fields have been alongside each other for quite some time and rely on each other for inspiration.

Systems biology is defined as an integrative, interdisciplinary approach to biological science that is built around the concept of close integration of computational methods, technology development (including omics) and global measurement and analysis of biological systems [16], and seem to be the future to this holistic or system-wide approach. Functional dynamics within systems biology as a field on its own greatly corresponds to the study of biological systems as the latter being dynamic is a key in its function. The challenge for functional dynamics and systems biology as a whole is to integrate not just biological complexity previously mentioned above, but also take into consideration external environmental interactions with the biological system at different levels which can significantly impact (through mutations, single nucleotide polymorphisms, change in network behavior, etc.) the biological system. From epigenomics/ epigenetics, we now know that genome-based information and its' permutations are not the only factor detrimental in health outcomes but environmental (including chemical, surroundings, lifestyle, social and economic) factors influence the interactome (complete repertories of interactions potentially encoded by the genome) [17], of human molecular physiology through the superimposition of the exposome (combined exposures from all sources that reach the internal chemical environment) [18]. With the exposome being in constant contact with the molecular complex system, one cannot appreciate and predict the functionality of the dynamics involved without taking into account the exposome.

\section{Personalized and Individualized}

With the completion of the human genome project and the post-genomics era, which started off with transcriptomics, proteomics and metabolomics among others pointed to the direction of a more individualized or personalized healthcare effort. As the user or patient became aware of knowledge through the influx of information present on the internet and (social) media and related online tools and 
digital libraries, the user demands personalized or preferentially individualized approaches towards his/her medical interventions and wants to be more involved in the decision making process [19]. Scientific data through research made it possible to predict at a systems level through simulations and models as well as through identification of early on biomarkers for diseases. Similarly prevention through early diagnosis (including family history, risk groups, genetic susceptibility, etc.) and understanding disease progression in an individual with reference samples as well as involving the person in the decision making process brought forward the concept of P4 (predictive, preventive, personalized and participatory) medicine [20]. However, this was not followed through contrary to the fact that there is now a gradual shift towards personalized interventions in the whole arena of healthcare [21], given the concept of 'one shoe does not fit all'. This transition also evolves from genomics focused personalized medicine to personalized medicine that takes all the relevant factors (including environmental) into account [21], shifting the paradigm from genomics to a systems approach in medicine and healthcare.

Given the dynamic nature of molecular life sciences as a large system (human) as mentioned above, this article aims to touch upon the challenge of encompassing environmental factors affecting biological molecular systems in scientific data and address the issue in personalized medicine of the bottlenecks of real-time integration of systems biology innovations in healthcare systems. 
Computational power in the form of ICT (information and communication technologies) was driven by large scale physics and commercial applications where medicine played a small role [22]. This is now changing as more ICT tools are being used and developed towards medicine. A revolution in ICT can potentially open the door for functional dynamics studies including systems biology applicability in healthcare like it did for other sectors.

The challenge remains largely for ICT to develop tools in the form of hardware and software to compute these already existing large data sets and ongoing generation of data taking into account the dynamic nature of the interactome [17], as well as all factors including socioeconomic, environmental and molecular determinants of health to implement the P4 medicine [20]. The EU pilot flagship project ITFoM (ICT Future of Medicine) is one of the six pilot projects in the 'European Future and Emerging Technologies flagships' and does take this into consideration. ITFoM aims to develop the virtual counterpart of an individual pulling the healthcare towards not just personalized medicine but individualized medicine, given the fact as described before that 'one shoe does not fit all' [21]. The idea is to first simulate and test drugs/medicines on the virtual patient (ICT-based replicas of molecular organization of individual humans) and/or gets advice from this developed ITFoM system and then executing the advice on the actual patient/individual. This can be accessed by both the individual and doctor for solutions. ITFoM and related projects of the "virtual patient" will develop an entirely new ICT that will enable real-time dynamic models of biochemical pathways, cells, tissues, diseases and ultimately, the entire human [22]. The strategy will be based on a common denominator replica [22]. Not only molecular data but all types of medical dataflow will be integrated in the system as well as environmental and lifestyle factors and will be a self-learning, follow-up system with reference datasets.

\section{Implementation}

However, like genome-based technologies and system biology, studies of functions of molecules or networks in dynamic environments although partly successful, do not see the implementations or direct use of these generated computational data applications in healthcare systems. Even if ICT does give a push in the right direction and we are able to develop the virtual patient there still seems to be a roadblock. This is given the assumption that the logical roadblock itself viz., the complexity of data and 
functionality in a dynamic environment is well understood or statistically significant and the environmental factors are acceptably taken into account. Furthermore, the ethical, legal, economic as well as privacy issues are considered. Although ICT and related projects may be the forerunners of taking into account to some extent ethical, legal, economic as well as privacy and governance issues there are implications beyond the obvious, which are generally an oversight and a common mistake of the academic-industrial complex [23].

ICT tools have been developed for medicine but its practical usage for most parts is far away. It can be also said that ICT tools developed for medicine, were limited to just molecular interactions and not taking into account general medical, environmental and lifestyle factors which greatly influence the outcome. ITFoM addresses these issues among others, however, from patterns $[24,25]$, in technological integration in healthcare systems [26], it seems evident technological diffusion is a long process [24]. ITFoM and related projects will set the stage for an ICT revolution in medicine. Learning from history, ICT innovations like health technologies, in general lack to capitalize on the issues of healthcare integration or translation, in a timely as well as effective and efficient manner $[24,27,28]$.

Public health and healthcare systems are a different system compared to tools (including technologies) developed for them. Coming back to the oversight or common mistake and the reason for the above mentioned pattern of delayed implementation, is that when developing technologies or knowledge discovery for transferring to healthcare systems, one generally neglects the health policy aspects of the healthcare system [29]. The commonly used activity for such translation [30,31], is technology transfer (TT), which is seen as the activity of the migration of academic discoveries to useful application in the development of marketable products or processes [32]. When valorizing new technologies focus is generally to the market itself and stays there. The push for healthcare implementation stagnates as a result and can be very long say 20 years [24]. Scientific merit or just evidence is not sufficient for real time uptake by healthcare. For example, the technology's clinical utility, which is a measure of the health care value provided by the technology [33], is of relevance, as well as the market authorization, reimbursement and insurance. Unheard terms like Health Needs Assessment (HNA), Health Technology Assessment (HTA) and Health Impact Assessment (HIA), collectively known as the Public Health Assessment Tools (PHAT) $[29,34,35]$, play an important role in healthcare implementation and are used by health professionals, doctors, decision makers or policy makers and are detrimental to insurance and reimbursement policies regarding a technology. 


\section{CHAPTER V}

HNA is a systematic method of reviewing the health issues facing a population, leading to agreed priorities and resource allocation that will improve health and reduce inequalities [34]. HTA is a multidisciplinary process that summarizes information about the medical, social, economic and ethical issues related to the use of a health technology in a systematic, transparent, unbiased, robust manner. Its aim is to inform the formulation of safe, effective, health policies that are patient focused and seek to achieve best value [36]. This corresponds to interventions and involvement in appraisals making HTA an influential tool to inform decision-making [29]. HIA is a combination of procedures, methods and tools by which a policy, program, or project may be judged as to its potential effects on the health of a population, and the distribution of those effects within the population [37]. In summary, HNA identifies health priorities for a given population, HTA evaluates the performance of health care technologies and HIA assesses the effects of policies, programs or projects on the population's health [34]. The above mentioned terms are generally neglected during technology transfer resulting in healthcare implementation delay [29].

Recently to resolve the bottleneck or roadblock a new framework called the LAL (Learning-AdaptingLeveling) model has been developed addressing healthcare implementation through and to the end of the technology transfer activity [29]. The LAL model's core (Figure 1) lies at the pseudo-parallel initiation of TT and PHAT through bilateral communication, feedback, public-private partnerships, and consultation between the two enterprises to adapt, stop or further the developing relevant technology based on its value to healthcare. Thus saving time and resources and accomplishes in real time by minimizing the delay of non or mis-communication between the different fields i.e. technology transfer and PHAT. Also, early on involvement of all stakeholders including the academic-industrial complex [23], health professionals, doctors, insurance companies, policy makers, patient groups, investors, etc. is within the framework of the model. In addition the 10 essential tasks of the Public Health wheel [38], which will ensure genome-based information and technologies, can be integrated into public health are covered. The LAL model brings two different fields of work for the first time together, i.e. technology transfer (TT) and the public health assessment tools (PHAT) and early on involvement of all stakeholders, public health integration and the value of information generated [29]. Ethical legal, social, economic aspects and clinical as well as personal utility [39-41], among others are covered in the model [29]. The model operates as a feedback mechanism to adapt and integrate new information and compensate through its collaborative communication network. It works as an overarching framework among frameworks within and between different enterprises. 


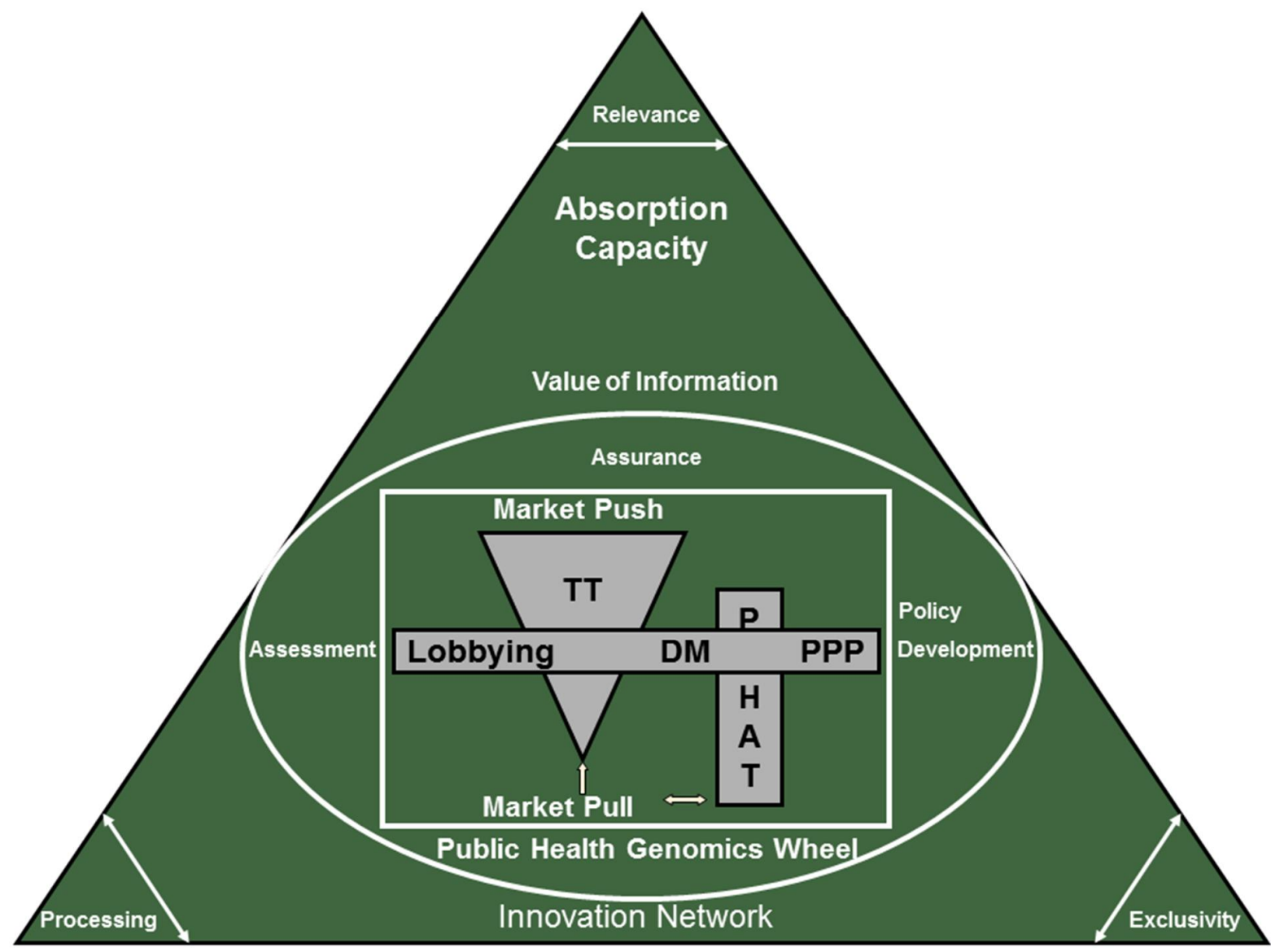

Figure 1: The Learning-Adapting-Leveling (LAL) model - The LAL model is a framework, which addresses the issue of real-time integration in healthcare systems. The added value of the model is that for the first time it brings together technology transfer and the public health assessment tools in parallel working together by crosstalk (via public-private partnerships or PPPs), giving rise to the innovation network, and in the process adapting the technology to public health and society needs. By the time the technology roles out of the TT pipeline, it is well suited to the conformity of PHAT, and to be taken up by decision makers (DM). This model takes into consideration, the 10 essential tasks of the public health wheel in the form of its 3 domains, namely policy development, assessment and assurance. The model also takes into account the Value of Information from the perspective of the developing technology's relevance to the end consumer, its processing ability or understandability to the user, and exclusivity like intellectual property rights, which may restrict its wide usage. This (innovation) network through PPPs has an optimal working function called the absorption capacity [29].

ITFoM also takes into consideration healthcare integration issues by following the LAL model in its implementation plan. Although the LAL model sets the stage for real-time uptake of relevant technologies and information data for healthcare implementation and the primordial soup for new policies in that direction, the development of policies favoring relevant uptake is an important aspect. Based on these polices which in part are contributed through recommendations of HTA reports, actual implementation and acceptance of health technologies or health information in healthcare settings are 


\section{CHAPTER V}

executed and for the technologies, reimbursement and market authorization is released. Policy tools in the form of guidelines have been developed by the Public Health Genomics European Network [42], (www.phgen.eu ) complimenting ITFoM and the LAL model.

The Public Health Genomics European Network (PHGEN II) is a European Commission DG SANCO (Directorate General for Health and Consumer Affairs) supported project and constitutes partners from all European Member States and observers. The aim of PHGEN II is to develop European best practice guidelines for quality assurance, provision and use of genome-based information and technologies by different stakeholders at an EU level [42]. The scope of PHGEN II is to assist EU Member States among others to develop relevant policies for-omics integration in healthcare. These guidelines [42] are a meta level guidance tool to further develop guidelines and are based around the 10 essential task of the Public Health Wheel [38], as can be seen from figure 2, which strives to integrate genome-based information and technologies into public health. This brings in the field of Public Health Genomics, which is the responsible and effective translation of genome-based knowledge and technologies into public policy and health services for the benefit of population health [43]. With final dissemination of these guidelines to all Member States and advocacy for policy implementation as well as set up of PHGEN National Task Forces will help towards healthcare implementation of ITFoM and the LAL model.

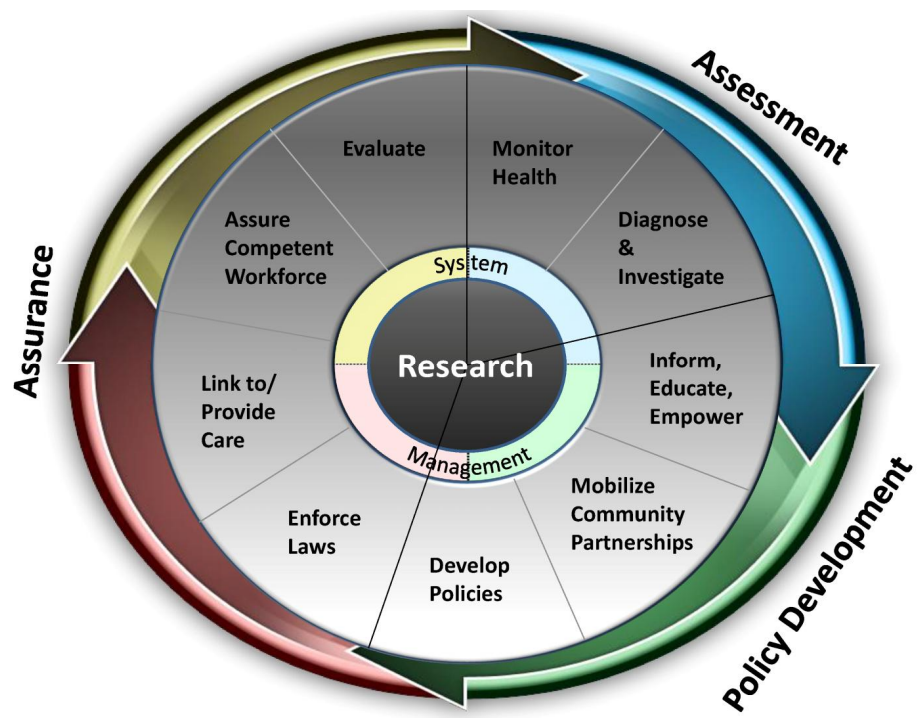

Figure 2: The 10 essential Public Health tasks - The 10 mentioned tasks are given in the pie chart divided over the 3 public health domains of assessment, policy development and assurance, with research being the core in all tasks. The European best practice guidelines developed by PHGEN were based around these 10 essential tasks [38]. 


\section{Discussion and Conclusions}

Although functional dynamics as just a field of study can exist as a research theme within systems biology, however for it to be useful to medicine, personalized medicine, personalized healthcare and healthcare as a whole, knowledge generated and subsequent relevant technologies developed need to be readily absorbed by healthcare systems in real-time. In order for that to happen the field while embarking on such an endeavor should not neglect health policy aspects and all stakeholders involved from an early on stage. The platform is set through ITFoM, the LAL model and PHGEN II for scientific, implementation and policy-wise respectively for healthcare uptake. However, these levels or steps can be achieved only once stability has been achieved with regard to the challenges functional dynamics face with biological complexity as previously stated. Moreover, environmental interactions add on to this seemingly chaotic, but actually synergistic complexity. ITFoM and related projects aim to address these two supposedly loose ends.

Another aspect of this implementation is the fact that there is a shift from the 'one shoe fits all' concept of medical classification and from stratified medicine to personalized and possibly individualized medicine given the uniqueness and similarities of individuals; and need to be taken into consideration when advocating for new policies. The LAL model and PHGEN II supports this shift in priorities and is already pre-existent within the core concept of ITFoM and related projects of the "virtual patient".

Systems biology coupled through the power of ICT seems to be the future of medical research and beyond given its systemic complexity and likely will benefit research and application of functional dynamics through magnifying its computational power and inclusion of environmental factors as a standard. Studying functions of molecules and networks without the exposome is indeed incomplete. This research will eventually push for personalized/individualized medical as well as health interventions in the future.

\section{Future Prospects}

Functional dynamics has a bright future given the incremental potential of ITFoM through systems biology. This is just not from a scientific point of view but given the framework of the LAL model and the policy in place through PHGEN II, active uptake by healthcare is a possibility. What remains to be seen is 


\section{| CHAPTER V}

how the ITFoM pilot project develops its models further and integrates data both scientific and environmental to its new ICT as well as developed infrastructure in hardware and software. The impact of the translation of this research and development into healthcare systems is hard to accurately predict given the semi-theoretical nature of what is currently implied, but what can be said is once accomplished it will greatly impact and revolutionize healthcare as a whole. It will change for sure the doctor-patient relationship. ITFoM tools can be used by both patients and doctors among others to be decided, however this will change the role each stakeholder plays and will bring it a new set of ethical, legal and socio-economic implications and organizational changes currently immeasurable. 


\section{References}

1. Alon U, Surette MG, Barkai N, Leibler S: Robustness in bacterial chemotaxis. Nature 1999, 397:168-171.

2. Yi TM, Huang Y, Simon MI, Doyle J: Robust perfect adaptation in bacterial chemotaxis through integral feedback control. Proceedings of the National Academy of Sciences of the United States of America 2000, 97:4649-4653.

3. Weng G, Bhalla US, Iyengar R: Complexity in biological signaling systems. Science 1999, 284:9296.

4. Levine K, Tinkelenberg A, Cross F: Progress in Cell Cycle Research. (eds. Meijer L, Guidet S, Lim Tung HY) Plenum, New York, 1995, 101-114.

5. Jordan JD, Landau EM, Iyengar R: Signaling networks: the origins of cellular multitasking. Cell 2000, 103:193-200.

6. Little JW, Shepley DP, Wert DW: Robustness of a gene regulatory circuit. The EMBO Journal 1999, 18:4299-4306.

7. Kitano H: Computation systems biology. Nature 2002, 420:206-209.

8. Goh KI, Cusick ME, Valle D, Childs B, Vidal M, Barabási AL: The human disease network. Proceedings of the National Academy of Sciences of the United States of America 2007, 104:8685-8690.

9. Altschul SF, Gish W, Miller W, Myers EW, Lipman DJ: Basic local alignment search tool. Journal of Molecular Biology 1990, 215:403-410.

10. Schatz MC, Delcher AL, Salzberg SL: Assembly of large genomes using second-generation sequencing. Genome Research 2010, 20:1165-1173.

11. Gusfield D: Algorithms on Strings, Trees and Sequences Computer Science and Computational Biology. USA: Cambridge University Press 1997.

12. Myers CL, Chiriac C, Troyanskaya OG: Discovering biological networks from diverse functional genomic data. Methods in Molecular Biology 2009, 563:157-175.

13. Goldberg DE: Genetic Algorithms in Search, Optimization and Machine Learning. 1st edn: Addison-Wesley Longman Publishing Co., Inc.; 1989.

14. Bishop CM: Neural Networks for Pattern Recognition. 1st edn: Oxford University Press; 1996. 


\section{CHAPTER V}

15. Navlakha S, Bar-Joseph Z: Algorithms in nature: the convergence of systems biology and computational thinking. Molecular Systems Biology 2011, 7:546.

16. Galas DJ, Hood L: Systems biology and emerging technologies will catalyze the transition from reactive medicine to Predictive, Personalized, Preventive And Participatory (P4) medicine. Interdisciplinary Bio Central 2009, 1:1-4.

17. Sanchez C, Lachaize C, Janody F, Bellon B, Röder L, Euzenat J, Rechenmann F, Jacq B: Grasping at molecular interactions and genetic networks in Drosophila melanogaster using FlyNets, an internet database. Nucleic Acids Research 1999, 27:90.

18. Rapport SM, Smith MT: Epidemiology. Environment and disease risks. Science 2010, 330:460461.

19. Sorenson K, Brand H: Health literacy: the essential catalyst for the responsible and effective translation of genome-based information for the benefit of the population health. Public Health Genomics 2011, 14:195-200.

20. Hood L, Friend SH: Predictive, personalized, preventive, participatory (P4) cancer medicine. Nature Reviews Clinical Oncology 2011, 8:184-187.

21. Cesuroglu T, Ommen Bv, Malats N, Sudbrak R, Lehrach H, Brand A: Public health perspective: from personalized medicine to personal health. Personalized Medicine 2012, 9:115-119.

22. Lehrach H, Sudbrak R, Boyle P, Pasterk M, Zatloukal K, Müller H, Hubbard T, Brand A, Girolami M, Jameson D, et al: ITFoM - The Future of Medicine. Procedia Computer Science 2011, 7:26-29.

23. Crow MM, Tucker C: The American research university system as America's de factor technology policy. Science and Public Policy 2001, 28:2-9.

24. Berwick DM: Dissseminating Innovations in Health Care. Journal of the Amercian Medical Association 2003, 289:1969-1970.

25. Murtagh J, Foerster V: Managing Technology Diffusion Discussion Paper. Canadian Agency for Drugs and Technologies in Health; 2009.

26. Booth-Clibborn N, Packer C, Stevens A: Health technology diffusion rates. Statins, coronary stents, and MRI in England. International Journal of Technology Assessment in Health Care 2000, 16:781-786.

27. Mcglynn EA, Asch SM, Adams J, Keesey J, Hicks J, DeCristofaro A, Kerr EA: The quality of health care delivered to adults in the United states. New England Journal of Medicine 2003, 348:26432644. 
28. Woolf SH: The meaning of Translational Research and Why it Matters. The Journal of the American Medical Association 2008, 299:211.

29. Lal JA, Bäumen TSid, Morré SA, Brand A: Public health and valorization of genome-based technologies: a new model. Journal of Translational Medicine 2011, 9:207.

30. Business Insights: Trends in Biotechnology Technology Transfer to 2014-Deal volume has doubled since 2008 [http://www.reports-research.com/168/d/2011/02/14/business-insightstrends-in-biotechnology-technologytransfer-to-2014-deal-volume-has-doubled-sin/]

31. Rasmussen E: The Process of New Venture Creation in a University Setting. Accompanying measures \& survival of new firms: between Darwinism and assistance. In; Montpellier. 2005

32. Mullins B, Crowe J: Technology Transfer: A Roadmap. In Book Technology Transfer: A Roadmap (Editor ed.^eds.), February edition. City: Association of College and Univeristy Auditors; 1999.

33. Clinical Utility of Personalised Medicine National Health and Medical Research Council. Australian Government; 2011.

34. Rosenköetter N, Vondeling H, Blancquaert I, Mekel OCL, Kristensen FB, Brand A: The Contribution of Health Technology Assessment, Health Needs Assessment, and Health Impact Assessment to the Assessment and Translation of Technologies in the Field of Public Health Genomics. Public Health Genomics 2011, 14:46-52.

35. Becla L, Lunshof JE, Gurwitz D, Bäumen TSId, Westerhoff HV, Lange BM, Brand A: Health technology assessment in the era of personalized health care. International Journal of Technology Assessment in Health Care 2011, 27:118-126.

36. HTA Definition [http://www.eunethta.eu/Public/About_EUnetHTA/HTA/]

37. Gothenburg Consensus Paper. Health Impact Assessment. Main concepts and suggested approach. In Book Gothenburg Consensus Paper. Health Impact Assessment. Main concepts and suggested approach (Editor ed.^eds.). City: HIA Gateway, West Midlands Public health Observatory; 1999.

38. Beskow LM, Khoury MJ, Baker TG, Thrasher JF: The integration of Genomics into public health research, policy and practice in the United States. Community Genetics 2001, 4:4.

39. Interim Report Quality Assurance. Public Heath Genomics European Network 2012.

40. Interim Report Provision. Public Heath Genomics European Network 2012.

41. Interim Report Use. Public Heath Genomics European Network 2012. 


\section{| CHAPTER V}

42. Brand A, Lal JA: European Best Practice Guidelines for Quality Assurance, Provision and Use of Genome-based Information and Technologies: the 2012 Declaration of Rome. Drug Metabolism and Drug Interactions 2012, 27:177-182.

43. Burke W, Khoury MJ, Stewart A, Zimmern RL: The path from genome based research to population health: Development of an international public health genomics network. Genetics in Medicine 2006, 8:453.

\section{Acknowledgements}

This project is supported by a grant from the European Commission PHGEN II (Duration period: June, 2009-May 2012 EU-Project No. 20081302). This work is also supported by the Canadian Institutes for Health Research (CIHR), CIHR Institute of Genetics, the CIHR Institute of Health Services and Policy Research through a grant (no. ETG92250), to the APOGEE-Net/CanGèneTest Research, and Knowledge Network on Genetic Services and Policy. We also like to extend our thanks to the research schools CAPHRI (program Public Health Genomics) and GROW of the Faculty of Health, Medicine and Life Sciences of Maastricht University for supporting this research. 
CHAPTER VI

CHLAMYDIA TRACHOMATIS INFECTIONS AND SUBFERTILITY: OPPORTUNITIES TO TRANSLATE HOST PATHOGEN GENOMIC DATA INTO PUBLIC HEALTH

Published as:

Lal JA, Malogajski J, Verweij SP, de Boer P, Ambrosino E, Brand A, Ouburg S, Morré SA.

Public Health Genomics 2013, 16:50-61. 
Chlamydia trachomatis (CT) infections in women can result in tubal pathology (TP). Worldwide 10-15\% of all couples are subfertile, meaning they did not get pregnant after 1 year. Part of the routine subfertility diagnostics is the Chlamydia Antibody Test (CAT) to decide for laparoscopy or not in order to diagnose TP. The CAT positive and negative predictive value is such that many unneeded laparoscopies are done and many TP cases are missed. Addition of host genetic markers related to infection susceptibility and severity could potentially improve the clinical management of couples who suffer from subfertility. In the present study, the potential translational and clinical value of adding diagnostic host genetic marker profiles on the basis of infection and inflammation to the current clinical management of subfertility was investigated. This review provides an overview of the current state of the art of host genetic markers in relation to $\mathrm{CT}$ infection, proposes a new clinical diagnostic approach, and investigates how the Learning-Adapting-Leveling model \{LAL, a public health genomic (PHG) model\} can be of value and provide insight to see whether these host genetic markers can be translated into public health. This review shows that the preliminary basis of adding host genetic marker profiles to the current diagnostic procedures of subfertility is present but has to be further developed before implementation into health care can be achieved. CT infection is an example in the field of PHG with potential diagnostic to be taken up in the future in the field of subfertility diagnosis with a time line for integration to be dependent on enhanced participation of many stakeholders in the field of PHG which could be advanced through the LAL model.

Keywords: Chlamydia trachomatis, Genomics, Host genetic markers, Learning-Adapting-Leveling model, Molecular diagnostics, Public health genomics, Stakeholders, Subfertility, Translation. 


\section{Chlamydia trachomatis Infection}

Chlamydia trachomatis is the most common bacterial sexually transmitted infection throughout the world. An estimated 89 million cases per year worldwide are reported. The infection is often asymptomatic resulting in patients not seeking treatment. Untreated urogenital $C$. trachomatis may give rise to late complications, including pelvic inflammatory disease, ectopic pregnancy and tubal pathology [1-4]. The clinical course of chlamydial infections is heterogeneous i.e. transmission, symptoms, clearance, and development of late complications differ per patient [5-7].

Sexually acquired $C$. trachomatis is an important public health concern for its effects on reproduction. Women who develop late complications, such as pelvic inflammatory disease or tubal pathology, suffer considerable morbidity and emotional distress, and are a socio-economic burden [8].

To prevent such outcomes, early diagnosis is important. Currently, screening for tubal pathology is performed via laparoscopy, a procedure, which is invasive and expensive, labor intensive and has a risk for surgical complications. This has resulted in extensive efforts to improve noninvasive diagnostic tests to decrease the risks of current screening methods.

Much research on bacterial components, clinical and environmental factors $[9,10]$ has been done, but no definitive correlates of late complications have been identified [11]. For a variety of infectious diseases (e.g. malaria, hepatitis and meningococcal infections), it has been shown that host genetics play a crucial role in susceptibility to and severity of disease [12-14]. To estimate the role of genetics in course of infection, twin studies are a powerful tool, and Bailey et al. [15] have shown that host genetic factors contribute almost $40 \%$ to the variation in clinical course of Chlamydia infection. These results establish the potential importance of genetic studies. Den Hartog et al. [16] showed, in a cohort of subfertile women, that single nucleotide polymorphisms (SNPs) in several pattern recognition receptors (PRRs) increase the risk of developing tubal pathology following a C. trachomatis infection.

The innate immune response is the first line of defense against a $C$. trachomatis infection. PRRs recognize components of the bacterium, and SNPs in these genes may affect the functionality of these PRRs and 


\section{| CHAPTER VI}

may, therefore, increase the risk for development of late complications. In addition, SNPs in genes coding for cytokines involved in immune responses may also be influential. Recently, our group [17] reviewed the role of SNPs in PRRs and cytokines in relation to susceptibility to a C. trachomatis infection.

It is important that these scientific findings are utilized in the clinic and incorporated in public health policy. The Learning-Adapting-Leveling (LAL) model [18] is a model of translating scientific data from the lab through the market and implementing it into public health policy; it will be discussed later in this review as a possible way to assimilate new findings in clinical settings.

\section{Outline of the Article}

The overarching aim of the current review is to determine if $\mathrm{C}$. trachomatis is one of the proof of principles in the field of public health genomics (PHG) with the potential to be taken up in the future in the field of subfertility diagnosis. Therefore, we provide an overview of the current state of the art on host genetic markers in relation to infection, propose a new clinical diagnostic approach in subfertility diagnostics based on this overview and describe how the LAL model (an integrated PHG model) can be of value to see if these host genetic markers can be translated from the lab to the market and implemented into public health.

\section{Overview of CT Host Genetic Determinants of Infection}

Several studies have shown the importance of host genetic variation on the clinical course of Chlamydia infections. This section will highlight recent findings, similar to a recent review [17], divided into detection of the pathogen Chlamydia by PRRs and the subsequent intercellular signaling by cytokines, with a focus on the innate immune system. Combined carriage of SNPs in so-called traits may exhibit a stronger influence on the course of Chlamydia infections, e.g. a reduced pathogen recognition capacity in multiple PRRs may result in higher susceptibility compared to the susceptibility when only one PRR has a reduced recognition capacity. Results for trait analyses are highlighted at the end of this section.

\section{Pattern Recognition Receptors}

Toll-Like Receptors (TLR)

TLRs are a much investigated group of receptors. Studies have shown that TLRs are essential in the host immune system by recognizing pathogenic components (pathogen associated molecular patterns (PAMPs) and danger associated molecular patterns (DAMPs) and inducing an immune response. These 
receptors are present on antigen presenting cells (APC) and epithelial cells; they reside both on the cell membrane and within cells. The TLR family has been studied in relation to various infectious and autoimmune diseases with varying associations $[17,19,20]$. TLRs 2,4 and 9 are well-researched TLR family members. TLR2 and TLR4, both trans-membrane pathogen receptors, recognize chlamydial peptidoglycan (PGN) and lipopolysaccharide (LPS), respectively. TLR9 is an intracellular receptor, recognizing $\mathrm{CpG}$ islands in bacteria.

Karimi et al. [19] investigated the role of 2 SNPs in TLR2 in C. trachomatis infected women and control groups: TLR2 -16934T>A (rs4696480) and TLR2 +2477G>A ( rs5743708). They did not find any association in TLR2 genotype distribution for both susceptibility to and severity of the infection. However, in haplotype analysis, they showed that haplotype TG was protective for developing tubal pathology. Laisk et al. [21] also evaluated the role of TLR2 +2477G >A ( $s 55743708$ ) in developing tubal pathology and found no association, confirming the results of the single SNP analyses of Karimi et al. [19].

Den Hartog et al. [22] studied TLR4 +896A>G (rs4986790). The genotype distribution of this SNP in subfertile women with or without C. trachomatis infection did not differ. However, women with this SNP and who were positive for C. trachomatis IgG and CHSP60 IgG had tubal pathology. Results of this study are highly specific, but have low sensitivity, since not all women with tubal pathology had this combination of C. trachomatis and cHSP60 serology, and TLR4 +896 mutation carriage. Laisk et al. [21] also investigated the role of this SNP in developing tubal pathology; they did not find any association. Taylor et al. [23] studied a different TLR4 polymorphism, rs1927911, and found that the mutant genotype increases the susceptibility to $C$. trachomatis infections. Similarly, they found that the TLR1 rs5743618 TT genotype increased susceptibility to Chlamydia infections [23].

Ouburg et al. [24] studied TLR9 SNPs in a murine model, in a cohort of Dutch Caucasian women visiting a STD outpatient clinic and a cohort of subfertile Dutch Caucasian women. The overall genotype distribution did not differ between groups. However, haplotype analyses showed, though not statistically significant, that distribution of TLR9 haplotype -1486 T (rs187084), -1237 C (rs5743836), +1174 G (rs352139), and +2848 A ( $r$ 352140) was more frequently found in women who developed tubal pathology. 


\section{| CHAPTER VI}

\section{C-C Chemokine Receptor Type 5 (CCR5)}

CCR5 is a chemokine receptor present on several immune cells, including monocytes, dendritic cells, microglial cells, T helper 1 cells, and macrophages. A 32-bp deletion within the CCR5 gene, CCR5 $\Delta 32$, results in premature termination of the protein, altering its function [25]. Barr et al. [26] demonstrated that this mutation has a protective effect against developing tubal pathology when both alleles are mutated. However, these results were not confirmed in a recent study [21] . For this inconsistency, the authors suggest that the ligand of CCR5, RANTES (CCL5), binds the CCR1 chemokine receptor as well, therefore inducing a normal response. Since they also used a different study population, they addressed the importance of group selection, and as a result this may be a reason for finding different outcomes [21].

\section{Mannose-Binding Lectin (MBL)}

MBL is important in the innate immune response. It binds to various carbohydrate structures of a.o. bacteria and either directly kills the pathogen or promotes phagocytosis [27]. Studies have shown that MBL inhibits a C. trachomatis infection [28]. Laisk et al. [21] investigated the role of 6 polymorphisms in the MBL2 gene, coding for MBL. They found that a hyperproduction haplotype of MBL2, HYA/HYA, was a risk factor for tubal pathology independent of a C. trachomatis infection. They also found this association, only smaller, in C. trachomatis infected patients with tubal pathology. They suggest that hyperproduction of MBL may affect epithelial cells within the genitourinary tract, inducing tubal pathology [21]. The low-producing MBL2 genotypes are associated with tubal pathology and adverse outcome of in vitro fertilization (IVF) treatment [29].

\section{Human Leukocyte Antigen (HLA)}

HLA codes for the major histocompatibility complex and thus, has an important function in the immune system. The HLA system has been linked to a variety of infectious diseases and disease outcomes. In the literature, a link between HLA -DQA1 * 0102 and HLA -DQB1 * 0602 alleles, and Chlamydia induced tubal pathology has been described [30].

Another studied receptor is the major histocompatibility complex class I chain-related A (MICA), present on a.o. natural killer cells. When its ligand binds, activating signals for natural killer cells increase. Allele MICA * 008 had a high negative correlation with C. trachomatis IgG antibodies. In this study, IgG antibodies were associated with tubal pathology. This group hypothesized that MICA alleles might play 
an important role in the development of tubal pathology. However, in the infertile women of their study, they could not establish an association between MICA alleles and tubal pathology with or without $\mathrm{C}$. trachomatis IgG antibodies [31].

\section{Cytokines}

A variety of cytokines have been associated with disease and disease outcomes. These cytokines have important immunoregulatory functions, and alterations in function may, therefore, influence immune responses. Several cytokines have been studied in women who developed tubal pathology. Some associations were found: IL10 -1082 A allele together with HLA -DQA1 * 0102 and HLA -DQB1 * 0602 alleles; these were associated with severe tubal pathology [30, 32]. Both the TNF- $\alpha-308 \mathrm{~A}$ allele and the IL6 CC genotypes were found to be associated with tubal pathology: the former as a risk factor and the latter as a protective factor for tubal pathology [32].

A statistically significant association between the IFNg +874 polymorphism and chlamydial tubal pathology was not found [32], nor for IL1B +3954, IL1B -511, and IL1RN gene polymorphisms [33] . However, a study performed in an ex vivo model showed that IL1, in the absence of its antagonist IL1RA, causes destruction of the ciliated cells in the Fallopian tubes [34]. In addition, SNPs in NLRP3, associated with hypoproduction of IL1 $\beta$, is involved in tubal pathology [35]. Due to these findings, one may hypothesize that SNPs influencing IL1 functionality may affect the development of tubal pathology and the rate of severity.

The mutant allele of the IL12B rs3212227 SNP is associated with increased susceptibility to tubal factor infertility and with a more severe progression of disease [36]. The same group also demonstrated that IL10 and IFNG genotypes affect the lympho-proliferative responses in Chlamydia infections [37].

\section{Trait Analyses}

Den Hartog et al. [16] investigated the role of 5 SNPs in 4 genes assumed to play a role in C. trachomatis infection. The investigated genes were TLR4, TLR9, CD14, and CARD15/NOD2. The risk for development of tubal pathology doubled if a patient had 2 or more SNPs within the studied genes, compared to 1 SNP. In addition, when investigating only 1 SNP in TLR4 or CD14 [38], no association with tubal pathology was found. Due to a small sample size, no statistical significance was observed in the trait analyses; a statistical trend, however, was observed. 


\section{| CHAPTER VI}

Ohman et al. [37] found that the combined carriage of specific IL10 and IFNG genotypes has an additive effect on the risk for Chlamydia infection. Atik et al. [39] demonstrated that combinations of SNPs affect the adverse that trachomatous trachiasis risk decreased 5 times with the combination of TNFA $(-308 \mathrm{~A})$, LTA (252A), VCAM1 (-1594C), and SCYA 11 (23T) minor allele, and the combination of TNFA (-308A), IL9 (113M), IL1B (5' UTR-T), and VCAM1 (-1594C). However, trachiasis risk increased 13.5 times with the combination of TNFA (-308G), VDR (intron G), IL4R (50V), and ICAM1 (56M) minor allele. Although these results are from ocular infections, one might hypothesize that similar effects might be observed in urogenital infections.

Although compelling, these results have to be confirmed in other studies, and additional SNPs have to be added in order to define the SNP profiles that are associated with and would help predict a patient's predisposition to Chlamydia infections and tubal pathology. This requires large cohorts which can be obtained via large consortia, in which different disciplines contribute to the overall goal.

\section{Consortium Approaches: EU Framework Program EpiGenChlamydia}

To perform large scale typing for the identification of genetic biomarkers, large and clinically welldefined cohorts are needed. Toward this end, a small consortium was founded in 2005 with Dutch, Belgian and American partners with expertise on clinical, epidemiological, bacterial, animal, immunological, and host immunogenetic studies to have an integrated approach to study $\mathrm{C}$. trachomatis infections, especially the clear inter-individual differences in the clinical course of infection. This consortium was named the ICTI consortium $[40,41]$. Members of the ICTI consortium applied for and obtained funding from the European Union as a large international consortium consisting of 20 partners, the EpiGenChlamydia (EGC) consortium [42].

This Chlamydia consortium was funded by the EU Framework Program 6 under the Coordination Actions in functional genomics research for a period of 2.5 years and provided its closure report in 2010. The aim of this consortium was to structure transnational research to such a degree that comparative genomics and genetic epidemiology on large numbers of unrelated individuals could be performed with future funding. This funding made it possible for 20 groups from Europe, Africa and the USA to participate (see http://www.EpigenChlamydia.eu for details). The overall goal of the EGC consortium was to accommodate the optimal environment to build and prepare a consortium to reliably determine the genetic predisposition to infection in both ocular and sexually transmitted C. trachomatis. This will allow 
the development of diagnostic tools that can determine an individual's predisposition to infection and the risk to develop late complications. Further, it was hoped that the knowledge generated through this effort would contribute to the understanding of the Chlamydia - host interaction, in order to allow the development of novel tools for the detection and treatment of and vaccine development for $\mathrm{C}$. trachomatis infections.

The EGC consortium has provided the final reports to the EU including state-of-the-art reports on the epidemiology of both ocular and sexually transmitted C. trachomatis infections [4], bacterial typing [43, 44] , immunogenetics [17] , SNP genotyping strategies, and sample validation. Two deliverables were of major importance for the future success in translation of immunogenetic markers and for obtaining new funding for a biobank, consisting of physical and virtual sample collections, and a data warehouse in which genotyping data together with clinical and demographical data is merged and accessible.

The partners working on ocular Chlamydia diseases coordinated by the London School of Hygiene and tropical medicine (David Mabey and Robin Bailey together with their Gambian partners) have already defined and secured 1,500 case-control pairs (total $n=3,000$ ). The scientific coordinator of the EGC consortium (S. Morré) together with Dutch collaborators have collected more than 7,000 specimens which are at present in use, while 10,000 specimens are available for further studies.

Currently, part of the consortium has obtained new funding from the EU based on Small-to-MediumEnterprise collaborations with universities. This EuroTransBio grant has as main goal to develop a diagnostic test on the basis of human genetics and C. trachomatis serology to better assess the presence of C. trachomatis -associated tubal damage in subfertile women. This consortium, ending in the beginning of 2015, is in progress of performing large scale analyses of human genetic variation to identify novel genetic markers that are able to stratify patients with tubal pathology. The identified SNPs in the PRR genes have already shown to be highly predictive for the development of tubal pathology. However, single SNPs do not provide a high enough predictive value for a diagnostic test. By combining multiple identified and novel SNPs in the PPR genes and genes in linked pathways, and exploiting them as susceptibility markers, a highly predictive test for tubal pathology-based subfertility can potentially be developed. This will be discussed further in the next section. 


\section{Improvement of Subfertility Diagnostics Based on Host Genetics}

Subfertility poses an enormous burden on healthcare and society throughout the world. Worldwide, $15 \%$ of couples trying to conceive suffer from subfertility $[45,46]$. In women, one of the major causes of female subfertility is tubal pathology [45]. In tubal pathology, C. trachomatis is the single most common cause for infertility [45].

From all subfertility problems in women, tubal damage is a common cause of infertility. It includes tubal obstruction and pelvic adhesions resulting from infection, endometriosis and previous surgery. The current diagnostic procedure for diagnosing this condition can be performed by sonohysterography/hysterosalpingo contrast sonography, hysterosalpingography (HSG), fertiloscopy, falloposcopy, or laparoscopy and dye hydrotubation, often using detection of IgG antibodies against C. trachomatis as first screenings tool.

Despite their effectiveness, the above-mentioned methods are costly and invasive [47] and not suitable for screening. There are several test methods available to assess the risk of $C$. trachomatis -associated tubal pathology in subfertile women. The reference standard for diagnosing tubal pathology in subfertile women is laparoscopy. However, laparoscopy has several disadvantages. First, it is an invasive, expensive procedure (on average 3,000 Euros, including additional costs) and requires general anesthesia. Furthermore, it holds a $1.5 \%$ risk of surgical complications (e.g. bleeding, infection).

Since it is widely recognized that a C. trachomatis infection is the single most common cause of tubal peritoneal damage (WHO task force on the prevention and management of infertility [48]), detecting evidence of infection using serology is noninvasive, simple and quick to perform [47]. As such, Chlamydia serology is often used as a first screening test for tubal damage in infertile women but has a limited sensitivity of $50-60 \%$.

Currently, women with subfertility are screened for a C. trachomatis using serology (see figure 1). Serologic testing (CAT: Chlamydia antibody testing) for $C$. trachomatis is based on micro immunofluorescence assays. Elevated titres of IgG are highly predictive for infection with $\mathrm{C}$. trachomatis. These serologic assays focus mainly on the major outer membrane protein $A$, which is an antigen present 
in the outer membrane of a chlamydial particle. When the serology outcome is negative, no further action is taken, and the couple is asked to try for one more year to get pregnant. Some women undergo HSG. If the outcome is negative (in most of the cases), they try as well to conceive for one more year. If positive (in around $5-7 \%$ of the cases) a laparoscopy will be performed, and up to $5 \%$ will have tubal pathology and will proceed to IVF procedures. Since HSG does not identify many new cases ( $5 \%$ of HSG positive cases), its positive predictive value is almost identical to serology. The low specificity of the test also causes misdiagnosis because women that are negative in the serology test may in fact have tubal pathology in up to $20 \%$ of cases (percentages based on the cohort described in ref [16], personal communication).

\section{Chlamydia}

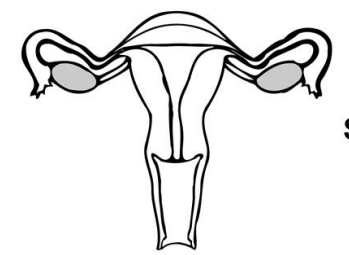

Subfertility

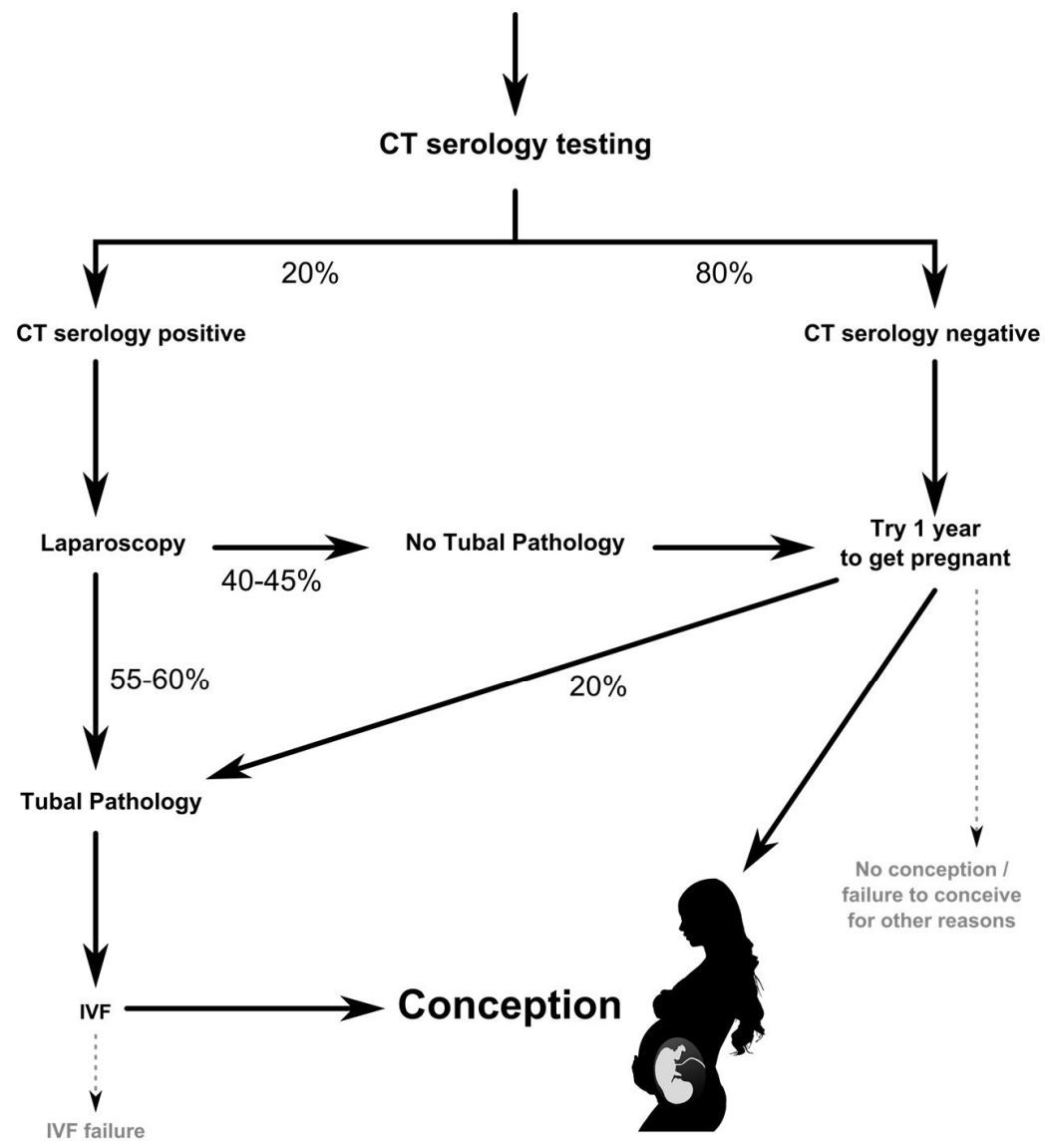

Figure 1: The relation between serology testing (Chlamydia antibody testing) and laparoscopy and the percentages of misdiagnosis. $\mathrm{CT}=\mathrm{C}$. trachomatis; IVF = in vitro fertilization.

If the serology outcome is positive, laparoscopy will be used for further diagnosis. When tubal damage is detected using laparoscopy, it is likely that an IVF procedure will be initiated to get pregnant. One of the drawbacks of serology is that it comes with limited sensitivity: $55-60 \%$ of the Chlamydia serology positive women actually have tubal pathology. Subsequently, this means that $40-45 \%$ of the women are serology positive and undergo laparoscopy while there is no tubal disease. In 


\section{CHAPTER VI}

conclusion, in the serology negative and positive groups, a significant percentage of the women get either unneeded laparoscopies or get misdiagnosed.

Given the prevalence of subfertility, each year an estimated number of 300,000 women visit the general practice with C. trachomatis -associated infertility in Europe. Using the current diagnostic procedures, there is still a substantial subset of women that are misdiagnosed. This poses an enormous psychological burden on these women. Additionally, there are tremendous economic costs associated with the disease.

Despite the rapid development in the field of human reproductive medicine, there is still a medical need for diagnostic tools that are able to stratify clinically relevant $C$. trachomatis infections. The diagnostic test envisioned should be able to predict $\mathrm{C}$. trachomatis -based tubal pathology and subsequent infertility by complementing serology in such a way that not only the sensitivity, but also the positive and negative predictive values increase significantly.

Epidemiological studies have demonstrated a $40 \%$ inheritable component for Chlamydia infections in humans [15]. This suggests the need for studies to identify which genes are responsible for this $40 \%$ component of risk. The innate immune system plays a pivotal role in the first recognition of Chlamydia and the subsequent immune response.

Since a panel of human PRRs are involved in the recognition of Chlamydia, we hypothesized and have shown that carrier traits (i.e. carrying multiple SNPs in multiple genes) result in a higher aberrant immune response as compared to single gene association studies. Subsequently, these traits synergistically increase risk for tubal pathology following C. trachomatis infection. Of 227 subfertile Dutch women, we performed genotyping of PRR genes TLR9, TLR4, CD14, and CARD15/NOD2 looking for common versus rare alleles [16] . Subfertility was defined based on laparoscopic grade of tubal pathology including extensive peri-adnexal adhesions and/or distal occlusion of at least one tube. Being a carrier of several rare alleles was more frequent in women with tubal pathology (who had elevated serum IgG titers against $C$. trachomatis). We showed that after $C$. trachomatis infection (these infections defined as CT IgG titers >32), subfertile women carrying >2 SNPs in PRR genes were at increased risk for tubal pathology compared to women carrying $<2$ SNPs (73 vs. $33 \%$ risk). This association was not found among women without IgG antibodies to $\mathrm{C}$. trachomatis. Thus, adequate recognition of $\mathrm{C}$. trachomatis 
by receptors in the genital tract is an important step in the immune response and may play a role in protecting the host against developing late sequelae following infection.

The single SNP frequencies of TLR2 SNPs did not alter the risk for tubal pathology in subfertile women, but, combined into specific haplotypes, carriage of haplotype I significantly reduced the risk of developing tubal pathology after a $C$. trachomatis infection $(p=0.015, O R=0.28)$. This haplotype also showed a significant trend in an inverse association with disease severity (asymptomatic > symptomatic $>$ tubal pathology; ptrend $=0.021$ ) [19]. In addition, PRRs, cytokines and chemokines play an essential role in the immunopathogenesis of C. trachomatis infections. The chemokine receptor, CCR5, is crucial for T cell activation and function, since its deficiency causes suppression of T cell responses. We showed that among patients with anti-chlamydial IgG responses, tubal pathology correlated with a low incidence of the CCR5 532 deletion (7\%), while women without tubal pathology had a higher incidence of the CCR5 432 deletion (31\%), as compared to controls (19\%) [26] . Thus, inflammation associated with CCR5 may predispose to development of complications of $C$. trachomatis infection. Recent findings show a similar pattern for CXCR5 [49].

Therefore, single SNPs, haplotypes, and eventually larger genetic traits based on genetic variation in multiple genes can potentially be used as susceptibility or severity markers for tubal pathology as a result of a C. trachomatis infection. By combining multiple SNPs in one diagnostic test, high predictive values can be achieved which should be suitable as a future companion diagnostic in the diagnosis and treatment strategy for subfertility. 


\section{Public Health Genomics Approached and Translation into Public Health}

Findings in the field of immunogenetics of $C$. trachomatis infections are of high relevance for public health and healthcare in general. Such results contribute to the understanding of infection with this agent, which is worldwide the leading cause of preventable blindness and the most prevalent sexually transmitted disease that is strongly associated with ectopic pregnancy, tubal infertility and pelvic inflammation. Furthermore, our findings provide new insights into the pathways that help explain individual heterogeneity in the clinical course of $\mathrm{C}$. trachomatis infection and the possible development of more targeted and personalized approaches in the prevention, diagnosis and treatment of disease.

To improve the health outcomes associated with C. trachomatis infections, there is an urgent need to timely translate immunogenetic findings into the healthcare system. The specialty having this task is called PHG, which is defined as 'the responsible and effective translation of genome-based knowledge and technologies into public policy and health services for the benefit of population health' [50] . In the context of $\mathrm{C}$. trachomatis infections, such a public health initiative has not been advanced, despite sufficient data and the significant need to do so. However, significant public health strategies need to be implemented very early after the discovery phase, and proofs of concept need to be obtained to promote a faster translational process not only from bench to bedside, but also from bedside to healthcare. Possible bottlenecks for implementation need to be identified.

Developing diagnostic tools based on host genetic predisposition can help determine an individual's risk (as well as late complications) of infection. However, moving forward with such a plan has hurdles along the path. We have seen that $\mathrm{C}$. trachomatis infections, symptoms and complications can differ between individuals on the basis of host genetic factors, ethnicity and environmental factors. And based on this review and on the literature, it seems likely that diagnostic assessment will allow for inclusion of a large number of case-specific variables (i.e. more stratified) that may even become 'truly' personalized in the near future with the incorporation information derived from dynamic fields of investigation such as systems biomedicine and epigenomics [51] . The major obstacle to implementation is not the CE (Conformtie Europeene) IVD or FDA approval of diagnostic application, but originates with the healthcare integration [52] and policy embedment processes. In general, a timely translation with direct 
implementation by the healthcare systems is low [53], which is illustrated by the large amount of data present in the literature $[54]$, patents $[55,56]$ and marketed products $[57,58]$ compared to technologies being used in hospitals $[53,59]$. In order to minimize failure, it is important that researchers take into account the policy aspect and the acceptance of diagnostic applications in the healthcare systems [52] itself, instead of just considering the market.

In order to move into the healthcare systems, it is important to think from the decision-making and policy implementation perspective. Health policy is generally developed through evidence-based interventions and around general public health instruments, such as Health Technology Assessment (HTA) among others [60, 61]. We use the term Public Health Assessment Tools (PHAT) [52] when we refer collectively to Health Needs Assessment, HTA and Health Impact Assessment. Health Needs Assessment is a systematic method of reviewing the health issues facing a population, leading to agreed upon priorities and resource allocation that will improve health and reduce inequalities [61]. HTA is a multi-disciplinary field of assessment that evaluates the medical, economic, social, legal, ethical, and other implications of the incremental value, diffusion and use of a technology in healthcare [62]. Health Impact Assessment is a combination of procedures, methods and tools by which a policy, program or project may be judged as to its potential effects on the health of a population and the distribution of those effects within the population. For simplicity, we will utilize HTA [63]. This assessment is generally done by HTA professionals in the form of systematic reviews of an emerging or new technology/tool with recommendations forwarded to decision-makers. Based on these recommendations, a technique, technology, tool, or process is implemented in the policy of healthcare, and these policy decisions determine the acceptability, the applicability in healthcare and the reimbursements. This in our observations has been generally neglected [52] by the academic-industrial complex [64]. Therefore, it is of uttermost importance to take into consideration the HTA process while developing a tool, diagnostic kit or technology, thus, in order to efficiently, effectively and in a timely manner introduce an innovation into the healthcare system. Although HTA or PHAT themselves are not sufficient when looking at the whole translational pipeline from bench to healthcare, HTA needs to be streamlined and integrated with the technology transfer process, which is the process of translating an idea into an innovative product on the market.

In the case of C. trachomatis, it can be said with some certainty that HLA, specific cytokines including IL10 and several TLRs play a role in infection and disease progression. These factors will likely vary 


\section{| CHAPTER VI}

between ethnical groups. For example, the most important TLR4 SNP, associated with tubal factor infertility in Caucasians, does not exist in Chinese people at all. Furthermore, the current needs of the population, relevant technologies on the market and prioritizing technologies based on applicability, the target audience, and stakeholder involvement including patient groups need to be taken into consideration. Also, the analytical validity (the ability of a test to accurately and reliably measure the genotype of interest) [65], clinical validity (the ability of the test to detect or predict the phenotype of interest) [65] and clinical utility (the likelihood that the test will lead to an improved outcome and incorporates assessment of the risk and benefits of genetic testing as well as economic evaluation) [65] of the diagnostic tool will have to be considered. In addition, by performing HTA, the economic, ethical, legal, and social implications will have to be thoroughly addressed in order to preempt any HTA related assessments by health policy-makers for technology integration. This collectively covers many aspects of HTA investigation.

Healthcare aims to improve health of the populations and that the perspective from which the healthcare decision-maker works is based on HTA recommendations. Therefore, at the end of the day, the approval of the technology lies in its clinical utility, since the analytical validity and clinical validity have been more or less addressed. Furthermore, equal weight is given to its ethical, economic, legal, and social implications (ELSI) in society. Generally, ethical, legal and economic issues are dealt with during the development of a diagnostic tool or technology; however, the social implications may be overlooked. This can be the downfall of the tool in healthcare implementation. Also, ELSI can be limited to one perspective: the industrial and not the population based perspective. Taking into account future developments such as 'truly' personalized medicine moving from clinical utility to personal utility and the use of 'personal-genome tests' [66] , current HTA as a tool for decision-making will be challenged [67] . Current HTA evaluates a technology on the population or subpopulation, but not on an individual level, which will be the need in the era of 'truly' personalized medicine.

When talking in terms of economics, reimbursement through insurance companies becomes important, which is sometimes addressed during the development of a technology. Financially stable patients with no need of reimbursement, early adopters or 'trendsetters' do not guarantee acceptance by decisionmakers as this does not represent the majority. HTA analysis is done based on the needs of the population as a whole and not based on a few elite. Therefore, these ethical questions have to be addressed here as well. HTA professionals generally prioritize technologies listed by them and investigate 
one or a few of them based on what they deem relevant to the current population need, which also has to be taken into account during the development of the diagnostic tool. This can be done via comparison to what already exists on how one can get a competitive advantage based on the current population need and through consultation with patient groups. This brings in another important aspect: the involvement of stakeholders. These stakeholders represent the needs of the population and should be given preference through the development of the diagnostic tool or technology. All these several aspects should be taken into account while developing a specific diagnostic kit for C. trachomatis subfertility, in order to decrease the chance of failure, if not guarantee its success in healthcare implementation. As a result among other factors, there is generally a delay in uptake and wide usage of diagnostic applications and technologies in healthcare systems and hospitals. Consequentially, by the time the technology or kit reaches the healthcare system, it becomes inferior in terms of efficiency and effectiveness, given the exponential growth of newer versions of emerging technologies on the market. The latter again goes through this delaying process.

Recently, a new framework or model [52] has been developed which addresses all the above mentioned issues and others not mentioned here with regard to healthcare implementation. The LAL model brings together for the first time 2 separate entities, namely technology transfer and PHAT in a pseudo-parallel initiation. Through this, the model promotes early on involvement of all stakeholders (including academics, industry, patient groups, insurance, policy makers, doctors, HTA professionals, etc.) via public-private partnership, consultation, bilateral communication, exchange of information, feedback loops, and relevant lobbying. The model ensures that through the technology transfer pipeline, all PHAT aspects are addressed, and it also encompasses the Public Health Genomics Wheel [68] as a reference frame which demonstrates the essential tasks of Public Health for integration of genome-based technologies ensuring all possible gaps are addressed. This ensures that by the time the technology or diagnostic application is developed, it conforms to the standards required by decision-makers based on population needs. This decreases the likelihood of rejection as a relevant tool for healthcare. The model also puts emphasis on the value of information (how much a decision-maker is willing to pay to come to a decision) [69]. This is in terms of the end-user clarity, including ease of use, relevance to the patient and doctors, and any legal issues restricting use of the diagnostic application widely. As a consequence of the value of information, adaptations in this case to the diagnostic application can be made accordingly. Through this process, by the time the technology or diagnostic application is developed, it meets all conditions of healthcare policy, therefore, facilitating timely uptake. We believe this overarching LAL 


\section{| CHAPTER VI}

model can ease the bottleneck of a real-time uptake by hospitals as well as help industry to come to an early-on strategic decision on the new technology and thus, save on resources [52]. The LAL model seems an appropriate tool and framework for the development of the Chlamydia diagnostic kit that will ensure all issues of healthcare, as well as public health conformity and industrial requirements, are met and addressed. As a result, by the time the diagnostic kit is ready, it can be made immediately available for widespread use throughout the healthcare system. This can help industry to tap into a generally wider consumer market than traditionally accessed as well as help decrease the burden of disease that more would result from delay in the technology. It becomes obvious, that public health approaches need to adjust to these developments. Thus, PHG in the future will be quite different from PHG in the past [51].

Rapid scientific advances in genomics and its application to epigenomics, microbiomics and systems biology not only contribute to the understanding of disease mechanisms, and to the characterization of each person's unique clinical, genomic, and environmental information, but also provide the option of new promising applications in patient and human health management during the whole life-course. In fact, what was just a decade ago a distant vision for a new era of public health, in which advances from the -omic sciences would be integrated into strategies aiming at benefiting population health, is now the soon-to-be realized development of effective personalized healthcare that will be 'truly' personalized medicine. The utility of most genetic tests and biomarkers is still not evidence-based enough. In the personalized medicine setting, the traditional assessment and evaluation tools are inadequate. We clearly face the need for a new paradigm because as we start to understand, for example, that what we call common complex diseases might be a sum of 'rare diseases'; we move from risk factors to individual pathways or networks, and from that perspective, we move from clinical utility to personal utility [70] . However, the real paradigm shift depends on the willingness to restructure policies and on the ability to train practitioners from various professions. P4 Medicine is a future vision defined by biologist Leroy Hood, and is short for 'Predictive, Preventive, Personalized, and Participatory Medicine' [71]. The premise of P4 Medicine is that, over the next 20 years, medical practice will be revolutionized by biotechnology, to manage a person's health, instead of manage a patient's disease. Although probably not around the next corner, there is a clear urgency to prepare healthcare systems and policy-makers in advance of the inevitable. 
The implementation of PHG requires increased concerted actions not only on the global (http://www.graphint.org), but also on the European level. The Public Health Genomics European Network (PHGEN II), which is funded by the General Directorate for Health and Consumer Protection (DG SANCO) (http://www.phgen.eu), initiated the National Task Forces on PHG in over 15 EU Member States. Due to these initiatives, the National Institutes of Public Health took a leading role in PHG in Finland, Belgium, the Netherlands, Croatia, Poland, or Germany. PHGEN II has developed 'European Best Practice Guidelines for Quality Assurance, Provision and Use of Genome-based Information and Technologies' [72], which will assist the European Member States. The LAL model has also been integrated in these guidelines. A major international research consortium called Information and Communication Technology for Future Medicine (ITFoM)' anticipates the medicine of the future, based on molecular, physiological, anatomical and environmental data from individual patients (http://www.itfom.eu). The 'ITFOM project' will create the entirely new ICT that will make it possible to make general models of human pathways, tissues, diseases, and ultimately of the human as a whole. Patient-individualized versions of ICT replica ('virtual patients') will be used to identify personalized prevention and therapy schedules and side effects of drugs [73]. The LAL model will play an important role in this project to make sure that by the time the technology is rolled out, it is adapted and conformed for real-time integration in healthcare.

\section{Conclusions and Future Prospects}

We investigated the potential translational and clinical value of adding diagnostic host genetic marker profiles relating to infection and inflammation to the current clinical diagnosis and management of subfertility, which is based on serology and laparoscopy. It is clear from a large body of evidence that host genetic factors play a role in the susceptibility to and severity of $C$. trachomatis infection, as shown by twin studies and many candidate gene studies. To bring this current host genetic work to the next level, large scale SNP typing and SNP identification in confirmation cohorts is essential and is in progress. This work will provide insight into what type of host genetic profile can help improve subfertility diagnoses and whether the added sensitivity, specificity, and positive and negative predictive value will realize the hope that carrier traits will significantly increase the ability to predict and identify those at greatest risk of severe complications from $C$. trachomatis infection. In addition, as shown by the LAL model, stakeholders have to be informed and participate early-on in the potential implementation of these findings, a major task and challenge for the field of public health genomics which can be realized through the LAL model. 
1. Manavi K: A review on infection with Chlamydia trachomatis. Best Pract Res Clin Obstet Gynaecol 2006; 20: 941-951.

2. Bébéar C, de Barbeyrac B: Genital Chlamydia trachomatis infections. Clin Microbiol Infect 2009; 15: 4-10.

3. Akande V, Turner C, Horner P, Horne A, Pacey A; British Fertility Society: Impact of Chlamydia trachomatis in the reproductive setting: British Fertility Society Guidelines for practice. Hum Fertil Camb) 2010; 13: 115-125.

4. Land JA, van Bergen JEAM, Morré SA, Postma MJ: Epidemiology of Chlamydia trachomatis infection in women and the cost-effectiveness of screening. Hum Reprod Update 2010; 16: 189204.

5. van Valkengoed IG, Morré SA, van den Brule AJ, Meijer CJLM, Bouter LM, van Eijk JT, Boeke AJ: Follow-up, treatment, and reinfection rates among asymptomatic Chlamydia trachomatis cases in general practice. Br J Gen Pract 2002; 52: 623-627.

6. Stamm WE: Diagnosis of Chlamydia trachomatis genitourinary infections. Ann Intern Med 1988; 108: 710-717.

7. Morré SA, Rozendaal L, van Valkengoed IG, Boeke AJ, van Voorst Vader PC, Schirm J, de Blok S, van Den Hoek JA, van Doornum GJ, Meijer CJ, van Den Brule AJ: Urogenital Chlamydia trachomatis serovars in men and women with a symptomatic or asymptomatic infection: an association with clinical manifestations? J Clin Microbiol 2000; 38: 2292-2296.

8. Brunham RC, Rey-Latino J: Immunology of Chlamydia infection: implications for a Chlamydia trachomatis vaccine. Nat Rev Immunol 2005; 5: 149-161.

9. Morré SA, Moes R, Van Valkengoed IG, Boeke JP, van Eijk JT, Meijer CJLM, Van den Brule AJ: Genotyping of Chlamydia trachomatis in urine specimens will facilitate large epidemiological studies. J Clin Microbiol 1998; 36: 3077-3078.

10. Molano M, Meijer CJLM, Morré SA, Pol R, van den Brule AJ: Combination of PCR targeting the VD2 of omp1 and reverse line blot analysis for typing of urogenital Chlamydia trachomatis serovars in cervical scrape specimens. J Clin Microbiol 2004; 42: 2935-2939.

11. den Hartog JE, Morré SA, Land JA: Chlamydia trachomatis -associated tubal factor subfertility: immunogenetic aspects and serological screening. Hum Reprod Update 2006; 12:719-730. 
12. Hill AV: Genetic susceptibility to malaria and other infectious diseases: from the MHC to the whole genome. Parasitology 1996; 112(suppl 112):S75-S84.

13. Frodsham AJ: Host genetics and the outcome of hepatitis B viral infection. Transpl Immunol 2005; 14: 183-186.

14. Smirnova I, Mann N, Dols A, Derkx HH, Hibberd ML, Levin M, Beutler B: Assay of locusspecific genetic load implicates rare Toll-like receptor 4 mutations in meningococcal susceptibility. Proc Natl Acad Sci USA 2003; 100: 6075-6080.

15. Bailey RL, Natividad-Sancho A, Fowler A, Peeling RW, Mabey DC, Whittle HC, Jepson AP: Host genetic contribution to the cellular immune response to Chlamydia trachomatis: heritability estimate from a Gambian twin study. Drugs Today (Barc) 2009; 45(suppl B):45-50.

16. den Hartog JE, Ouburg S, Land JA, Lyons JM, Ito JI, Peña AS, Morré SA: Do host genetic traits in the bacterial sensing system play a role in the development of Chlamydia trachomatis associated tubal pathology in subfertile women? BMC Infect Dis 2006; 6: 122.

17. Morré SA, Karimi O, Ouburg S: Chlamydia trachomatis : identification of susceptibility markers for ocular and sexually transmitted infection by immunogenetics. FEMS Immunol Med Microbiol 2009; 55: 140-153.

18. Lal JA, Schulte in den Bäumen T, Morré SA, Brand A: Public health and valorization of genomebased technologies: a new model. J Transl Med 2011; 9: 207.

19. Karimi O, Ouburg S, de Vries HJ, Peña AS, Pleijster J, Land JA, Morré SA: TLR2 haplotypes in the susceptibility to and severity of Chlamydia trachomatis infections in Dutch women. Drugs Today (Barc) 2009; 45(suppl B):67-74.

20. van der Paardt M; Crusius JBA; de Koning MHMT, Morré SA, van de Stadt RJ, Dijkmans BA, Peña AS, van der Horst-Bruinsma IE: No evidence for involvement of the Toll-like receptor 4 (TLR4) A896G and CD14- C260T polymorphisms in susceptibility to ankylosing spondylitis. Ann Rheum Dis $2005 ; 64: 235-238$.

21. Laisk T, Peters M, Saare M, Haller-Kikkatalo K, Karro H, Salumets A: Association of CCR5, TLR2, TLR4 and MBL genetic variations with genital tract infections and tubal factor infertility. J Reprod Immunol 2010; 87: 74-81.

22. den Hartog JE, Lyons JM, Ouburg S, Fennema JS, de Vries HJ, Bruggeman CA, Ito JI, Peña AS, Land JA, Morré SA: TLR4 in Chlamydia trachomatis infections: knockout mice, STD patients and women with tubal factor subfertility. Drugs Today (Barc) 2009; 45(suppl B):75-82. 


\section{| CHAPTER VI}

23. Taylor BD, Darville T, Ferrell RE, Kammerer CM, Ness RB, Haggerty CL: Variants in tolllike receptor 1 and 4 genes are associated with Chlamydia trachomatis among women with pelvic inflammatory disease. J Infect Dis 2012; 205: 603-609.

24. Ouburg S, Lyons JM, Land JA, den Hartog JE, Fennema JS, de Vries HJ, Bruggeman CA, Ito JI, Peña AS, Lundberg PS, Morré SA: TLR9 KO mice, haplotypes and CPG indices in Chlamydia trachomatis infection. Drugs Today (Barc) 2009; 45(suppl B):83-93.

25. Mueller A, Strange PG: The chemokine receptor, CCR5. Int J Biochem Cell Biol 2004; 36:35-38.

26. Barr EL, Ouburg S, Igietseme JU, Morré SA, Okwandu E, Eko FO, Ifere G, Belay T, He Q, Lyn D, Nwankwo G, Lillard JW Jr, Black CM, Ananaba GA: Host inflammatory response and development of complications of Chlamydia trachomatis genital infection in CCR5-deficient mice and subfertile women with the CCR5 $\triangle 32$ gene deletion. J Microbiol Immunol Infect 2005; 38: 244-254.

27. Turner MW: Mannose-binding lectin (MBL) in health and disease. Immunobiology 1998; 199: 327-339.

28. Swanson AF, Ezekowitz RA, Lee A, Kuo CC: Human mannose-binding protein inhibits infection of HeLa cells by Chlamydia trachomatis. Infect Immun 1998; 66: 1607-1612.

29. Laisk T, Peters M, Salumets A: Mannosebinding lectin genotypes: potential role in tubal damage and adverse IVF outcome. J Reprod Immunol 2011; 92: 62-67.

30. Kinnunen AH, Surcel HM, Lehtinen M, Karhukorpi J, Tiitinen A, Halttunen M, Bloigu A, Morrison RP, Karttunen R, Paavonen J: HLA DQ alleles and interleukin-10 polymorphism associated with Chlamydia trachomatis -related tubal factor infertility: a case-control study. Hum Reprod 2002; 17: 2073-2078.

31. Mei B, Luo Q, Du K, Huo Z, Wang F, Yu P: Association of MICA gene polymorphisms with Chlamydia trachomatis infection and related tubal pathology in infertile women. Hum Reprod 2009; 24: 3090-3095.

32. Ohman H, Tiitinen A, Halttunen M, Lehtinen M, Paavonen J, Surcel HM: Cytokine polymorphisms and severity of tubal damage in women with Chlamydia -associated infertility. J Infect Dis 2009; 199: 1353-1359.

33. Murillo LS, Land JA, Pleijster J, Bruggeman CA, Peña AS, Morré SA: Interleukin-1B (IL-1B) and interleukin-1 receptor antagonist (IL-1RN) gene polymorphisms are not associated with tubal pathology and Chlamydia trachomatis-related tubal factor subfertility. Hum Reprod 2003; 18: 2309-2314. 
34. Hvid M, Baczynska A, Deleuran B, Fedder J, Knudsen HJ, Christiansen G, Birkelund S: Interleukin-1 is the initiator of Fallopian tube destruction during Chlamydia trachomatis infection. Cell Microbiol 2007; 9:2795-2803.

35. Wang W, Stassen FR, Surcel HM, Ohman H, Tiitinen A, Paavonen J, de Vries HJ, Heijmans R, Pleijster J, Morré SA, Ouburg S: Analyses of polymorphisms in the inflammasome-associated NLRP3 and miRNA-146A genes in the susceptibility to and tubal pathology of Chlamydia trachomatis infection. Drugs Today (Barc) 2009; 45(suppl B):95-103.

36. Ohman H, Bailey R, Natividad A, Ragoussis J, Johnson LL, Tiitinen A, Halttunen M, Paavonen J, Surcel HM: Effect of IL12A and IL12B polymorphisms on the risk of Chlamydia trachomatis induced tubal factor infertility and disease severity. Hum Reprod 2012; 27: 2217-2223.

37. Ohman H, Tiitinen A, Halttunen M, Paavonen J, Surcel HM: Cytokine gene polymorphism and Chlamydia trachomatis-specific immune responses. Hum Immunol 2011; 72:278-282.

38. Ouburg S, Spaargaren J, den Hartog JE, Land JA, Fennema JS, Pleijster J, Peña AS, Morré SA; ICTI consortium: The CD14 functional gene polymorphism $-260 C>T$ is not involved in either the susceptibility to Chlamydia trachomatis infection or the development of tubal pathology. BMC Infect Dis 2005; 5: 114.

39. Atik B, Skwor TA, Kandel RP, Sharma B, Adhikari HK, Steiner L, Erlich H, Dean D: Identification of novel single nucleotide polymorphisms in inflammatory genes as risk factors associated with trachomatous trichiasis. PLoS One 2008; 3:e3600.

40. Morré SA, Spaargaren J, Ossewaarde JM, Land JA, Bax CJ, Dörr PJ, Oostvogel PM, Vanrompay D, Savelkoul PH, Pannekoek Y, van Bergen JE, Fennema HS, de Vries HJ, Crusius JB, Peña AS, Ito JI, Lyons JM: Description of the ICTI consortium: an integrated approach to the study of Chlamydia trachomatis infection. Drugs Today 2006; 42(suppl A):107-114.

41. Lyons JM, Ouburg S, Morré SA: An integrated approach to Chlamydia trachomatis infection: the ICTI Consortium, an update. Drugs Today 2009; 45(suppl B):15-23.

42. Morré SA, Ouburg S, Peña AS, Brand A: The EU FP6 EpiGenChlamydia Consortium: contribution of molecular epidemiology and host-pathogen genomics to understanding Chlamydia trachomatis -related disease. Drugs Today 2009; 45(suppl B):7-13.

43. Klint M, Fuxelius HH, GoldkuhI RR, Skarin H, Rutemark C, Andersson SG, Persson K, Herrmann B: High-resolution genotyping of Chlamydia trachomatis strains by multilocus sequence analysis. $J$ Clin Microbiol 2007; 45:1410-1414. 


\section{| CHAPTER VI}

44. Pedersen LN, Herrmann B, Møller JK: Typing Chlamydia trachomatis : from egg yolk to nanotechnology. FEMS Immunol Med Microbiol 2009; 55: 120-130.

45. Evers JL: Female subfertility. Lancet 2002; 360:151-159.

46. Broeze KA, Opmeer BC, Van Geloven N, Coppus SF, Collins JA, Den Hartog JE, Van der Linden PJ, Marianowski P, Ng EH, Van der Steeg JW, Steures P, Strandell A, Van der Veen F, Mol BW: Are patient characteristics associated with the accuracy of hysterosalpingography in diagnosing tubal pathology? An individual patient data meta-analysis. Hum Reprod Update 2011; 17: 293-300.

47. Akande VA, Hunt LP, Cahill DJ, Caul EO, Ford WC, Jenkins JM: Tubal damage in infertile women: prediction using Chlamydia serology. Hum Reprod 2003; 18: 1841-1847.

48. Tubal infertility: serologic relationship to past chlamydial and gonococcal infection. WHO Task Force on the Prevention and Management of Infertility. Sex Transm Dis 1995; 22:71-77.

49. Jiang J, Karimi O, Ouburg S, Champion Cl, Khurana A, Liu G, Freed A, Pleijster J, Rozengurt N, Land JA, Surcel HM, Tiitinen A, Paavonen J, Kronenberg M, Morré SA, Kelly KA: Interruption of CXCL13CXCR5 axis increases upper genital tract pathology and activation of NKT cells following chlamydial genital infection. PLoS One 2012; 7:e47487.

50. Burke W, Khoury MJ, Stewart A, Zimmern RL: The path from genome-based research to population health: development of an international public health genomics network. Genet Med 2006; 8: 451-458.

51. Brand A: Public health genomics - public health goes personalized? Eur J Public Health 2011; 21: 2-3.

52. Lal JA, Schulte in den Bäumen T, Morré SA, Brand A: Public health and valorization of genomebased technologies: a new model. J Transl Med 2011; 9:e207.

53. Berwick DM: Disseminating innovations in health care. JAMA 2003; 289: 1969-1970.

54. Peng W: Trends in the biotech literature 2009. Nat Biotechnol 2010; 28: 887.

55. Snyder M, Cook-Deegan B: DNA Patent Database Statistics: 2010. DNA Patent Database. 2011.

56. Catalogue of Biotechnology Patents in the Mercosur. BIOTECHSUR. 2008. http://docs.biotecsur.org/informes/en/inventario/9_patentes_ms.pdf.

57. HM Government: Strength and Opportunity. The landscape of the medical technology, medical biotechnology and industrial biotechnology sectors in the UK. 2009; 11-12, 28, 34, 38, 40.

58. US Biotech Market Analysis: RNCOS Industry Research Solutions. 2010

59. Murtagh J, Foerster V: Managing Technology Diffusion-Discussion Paper. Canadian Agency for Drugs and Technologies in Health. 2009; 4: 7-8, 10. 
60. Brand A, Brand $H$, Schulte in den Bäumen $T$ : The impact of genetics and genomics on public health. Eur J Hum Genet 2008; 16: 5-13.

61. Rosenkötter N, Vondeling H, Blancquaert I, Mekel OCL, Kristensen FB, Brand A: The contribution of health technology assessment, health needs assessment, and health impact assessment to the assessment and translation of technologies in the field of public health genomics. Public Health Genomics 2010; 14: 43-52.

62. Battista RN: Expanding the scientific basis of health technology assessment: a research agenda for the next decade. Int J Technol Assess Health Care 2006; 22: 275-280.

63. Health Impact Assessment: Main concepts and suggested approach Gottenburg consensus paper. Brussels: European Center for Health Policy. 1999; 4.

64. Crow MM, Tucker C: The American research university system as America's de facto technology policy. Sci Public Policy 2001; 28: 2-9.

65. Haddow J, Palomaki G: ACCE: A model process for evaluating data on emerging genetic tests; in Khoury M, Little J, Burke W (eds): Human Genome Epidemiology. Oxford, Oxford University Press, 2004, pp 217-233.

66. Brand A: The future is being built today: facing the era of integrative genomics, personal-genome tests and personalized healthcare - a need for integrative minds? Eur J Hum Genet 2009; 17: 977-978.

67. Becla L, Lunshof JE, Gurwitz D, Schulte in den Bäumen T, Westerhoff HV, Lange BM, Brand A: Health technology assessment in the era of personalized health care. Int J Technol Assess Health Care 2011; 27: 118-126.

68. Beskow LM, Khoury MJ, Baker TG, Thrasher JF: The integration of Genomics into public health research, policy and practice in the United States. Community Genet 2001; 4: 2-11.

69. Oestreich T: The value of information Part 1-The Framework. Journal of Management Excellence: The Value of Information 2010; 9:7-10. http://www.oracle.com/us/solutions/businessintelligence/064068.pdf.

70. Brand A, Ambrosino E, Knoppers BM: Public Health Genomics journal: adjusting the agenda to future needs. Public Health Genomics 2011; 14: 125-126.

71. Hood L: A Doctor's Vision of the Future of Medicine. Newsweek, June 27, 2009.

72. Brand A, Lal JA; Public Health Genomics European Network: European Best Practice Guidelines for Quality Assurance, Provision and Use of Genome-based Information and Technologies - the 2012 Declaration of Rome. Drug Metabol Drug Interact 2012; 27: 177-182. 


\section{| CHAPTER VI}

73. Lehrach H, Sudbrak R, Boyle P, Pasterk M, Zatloukal K, Müller H, Hubbard T, Brand A, Jameson D, Westerhoff HV: ITFoM - the IT future of medicine. Procedia Comput Sci 2011; 7: 26-29.

\section{Acknowledgement}

The authors thank the Public Health Genomics European Network (PHGEN) for commissioning work on assessment instruments in Public Health Genomics within the European Union. This work was supported by two grants of the European Commission (DG SANCO, Agreement PHGEN I 2005313 and Agreement PHGEN II 20081302). The aim of this work is in line with the European EpiGenChlamydia Consortium which was supported by the European Commission within the Sixth Framework Program through contract LSHG-CT-2007-037637. See http://www.Epi-GenChlamydia.eu for more details about this consortium. 
CHAPTER VII

EUROPEAN BEST PRACTICE GUIDELINES FOR QUALITY ASSURANCE, PROVISION AND USE OF GENOME-BASED

INFORMATION AND TECHNOLOGIES: THE 2012

DECLARATION OF ROME

Published as:

Brand A, Lal JA.

Drug Metabolism and Drug Interactions 2012, 27(3):177-182. 


\section{Introduction}

Genomics is a highly dynamic field and, as such, represents a moving target for public health. Public health is shifting from a focus on the population towards an emphasis of the individual as a means of supporting the well-being of the population. In particular, we are entering the era of predictive, personalized, preemptive and participatory (P4) medicine supported by advanced technological infrastructure. These changes represent a paradigm shift in our approach to healthcare and will go handin-hand with a major reclassification of diseases. The challenge now is to understand how all of these changes will impact public health and how to ensure that they are translated effectively into benefits for individual citizens and society as a whole. Thus, there is a need to develop guidelines aiming not to close doors. Instead, the goal is to create a vision that allows for flexibility and adaptability in their implementation in order to have a maximum impact on health, the healthcare infrastructure, health technologies and economic growth in the health sector.

Therefore, the European Commission asked to develop "European Best Practice Guidelines for Quality Assurance, Provision and Use of Genome-based Information and Technologies" to support the Member States (and other relevant stakeholders) to more efficiently and effectively work together at a European level in addressing the challenges deriving from emerging genome-based information and technologies and to prepare for the paradigm shift of personalized healthcare in time. The implementation of the concept of public health genomics being the responsible and effective translation of genome-based knowledge and technologies for the benefit of population health requires modifications of public health and health governance systems on all levels.

The Public Health Genomics European Network (PHGEN II) fulfills this task. It is an EU DG SANCO (European Union Directorate-General for Health and Consumers) funded and European Agency for Health and Consumers (EAHC) issued project (EU Project No. 20081302, 2009 - 2012), which recently produced the first edition of the "European Best Practice Guidelines for Quality Assurance, Provision and Use of Genome-based Information and Technologies". The guidelines will assist all EU Member States, Applicant and EFTA-European Economic Area (EEA) countries with evidence-based guidance on the timely and responsible integration of genome-based information and technologies into healthcare systems for the benefit of population health. They build on the extensive work of the Public Health 
Genomics European Network (PHGEN, www.phgen.eu) being the cornerstone in the development of public health genomics in Europe. Whereas PHGEN I (DG SANCO 2006 - 2008) identified the need for European best practice guidelines ("mapping exercise"), PHGEN II developed the first edition of these European best practice guidelines using the concept of "genome-based information and technologies" (Bellagio- Model), which PHGEN I established as a scientific benchmark in Europe. In this concept, genome-based information is very holistic and includes not only all "omics" data but also environmental, socioeconomic and lifestyle factors, as well as information on health systems. The process of the development of guidelines was in line with international standards and acknowledges the diversity and cultural differences in Europe. Key experts, such as public health experts, EU lawyers, human geneticists, ethicists, systems biologists, Health Technology Assessment (HTA) experts, representatives from the private sector and patient groups, as well as policy makers had been involved in PHGEN II.

On 19 and 20 April 2012, experts from across the field of public health genomics representing key European and national organizations and institutions from policy making, academia and private sector came together at the final PHGEN II meeting in Rome - amongst them the European Society for Pharmacogenomics and Theranostics (ESPT) and the European Medicines Agency (EMA) - to discuss the future of public health genomics and to endorse the Declaration of Rome on 19 April 2012, a summary of the "European Best Practice Guidelines for Quality Assurance, Provision and Use of Genome-based Information and Technologies".

\section{Preamble}

In the past decade, we have witnessed major changes in our approach to public health, not only in terms of research and practice but also the policies that support it. New insights into our individual biological make-up are being obtained from genomics, proteomics, epigenomics, microbiomics and other "omics" technologies. As these data are integrated through the use of information and communication technologies (ICT), we are on the brink of achieving an understanding of the systems biology of human health and disease that also incorporates environmental contributions. In this way, we can begin to envisage new approaches to the promotion and management of human health across the entire life course of an individual. Indeed, we can now consider a future involving truly personalized healthcare in which technological advances are placed at the service of population health. The evidence we now require to demonstrate the benefit of new technologies will need to follow a new paradigm, however. What is required is an assessment of individual benefit rather than overall effects in large populations or 


\section{| CHAPTER VII}

even subpopulations of patients. Thus, public health assessment and evaluation tools must now address concepts, such as personal rather than clinical utility.

In light of these changes, PHGEN II recognizes that:

- Common complex diseases can be considered in terms of a constellation of "rare" diseases, each of which reflects a complex biological system.

- We are moving away from a traditional classification of disease and towards groups of shared pathology that can be described as "diseasomes" or disease nodes.

- We are moving away from a focus on risk factors within biostatistical models of populations and towards an emphasis of individual pathways or networks.

- It is time to emphasize personal rather than clinical utility.

The use of genome-based knowledge and technologies will increase the quality of life of European citizens. Predictive, personalized, preemptive and participatory (P4) medicine serves as a blueprint for the Public Health Genomics European Network (PHGEN II) to prepare healthcare systems and policy makers for this paradigm shift in our approach to healthcare. This shift will lead to a fundamental change not only in knowledge but also in our view of ourselves and the way we are living. As a result, society must be willing to restructure policies and support knowledge transfer to maximize benefit to public health.

\section{Information and Communication Technologies}

The increasing role of ICT in healthcare is driven by improved technological options. Combined genomic and phenotypic analysis has become possible owing to advances in technologies to support data analysis and modeling. The complexity of the task when applied to diagnosis and therapy, however, demands algorithms and mathematical models to reduce uncertainties. As a result, efforts are now being made to generate computational models of individual persons ("virtual twins"). Such models can be used to follow individuals throughout their lifetime and enable health professionals to optimize all types of health interventions. In this way, it becomes possible to improve the safety, quality, effectiveness and efficiency of healthcare services. In addition, by following individuals rather than remaining tied to a given healthcare system, it will enable citizens to handle and access personal health-related data whenever needed. 


\section{Genome-based information and technologies}

PHGEN II builds on the definitions that were developed and agreed in PHGEN I, such as the glossary on public health genomics and the status of genetic information and genetic testing. The definitions provided by PHGEN II take into account the most recent developments in the fields of genomics, systems biology and systems medicine, which provide the evidence base for the "European Best Practice Guidelines for Quality Assurance, Provision and Use of Genome-based Information and Technologies" (see Figure 1: Research for new insights and innovative solutions to health problems). The terms genome-based information (GBI) and genome-based technologies (GBT) are encompassed by the term genome-based information and technologies (GBIT).

These definitions address the following areas:

- genetics, genomics, functional genomics, systems biology and systems medicine, and the distinctions between them;

- genetic disease, genomic disease and systems biology disease, and the distinctions between them;

- the differences between generic, cohort-based, personalized and truly individualized medicine. Based on these definitions, systems medicine is understood through the integration of genetics, genomics, functional genomics and systems biology.

\section{Rationale for the European Best Practice Guidelines for Quality Assurance, Provision and Use of Genome-based Information and Technologies}

The ultimate objective of PHGEN II is to enable informed decisions at the macro-, meso- and micro-levels regarding quality assurance, provision and use of emerging GBIT. The means chosen by PHGEN II to achieve this is the preparation of European best practice guidelines to support this decision-making process now and in the future. However, meta-level guidance is also needed. This meta-level guidance can be achieved by ensuring that the ten essential public health tasks, as described within the public health wheel or public health trials (assessment, policy development, assurance) can be adequately fulfilled in each jurisdiction on the basis of a common understanding of best practice guidelines for each task (see Figure 1). Within these best practice guidelines, translational research considerations are combined with system management under the concept of public health genomics. 


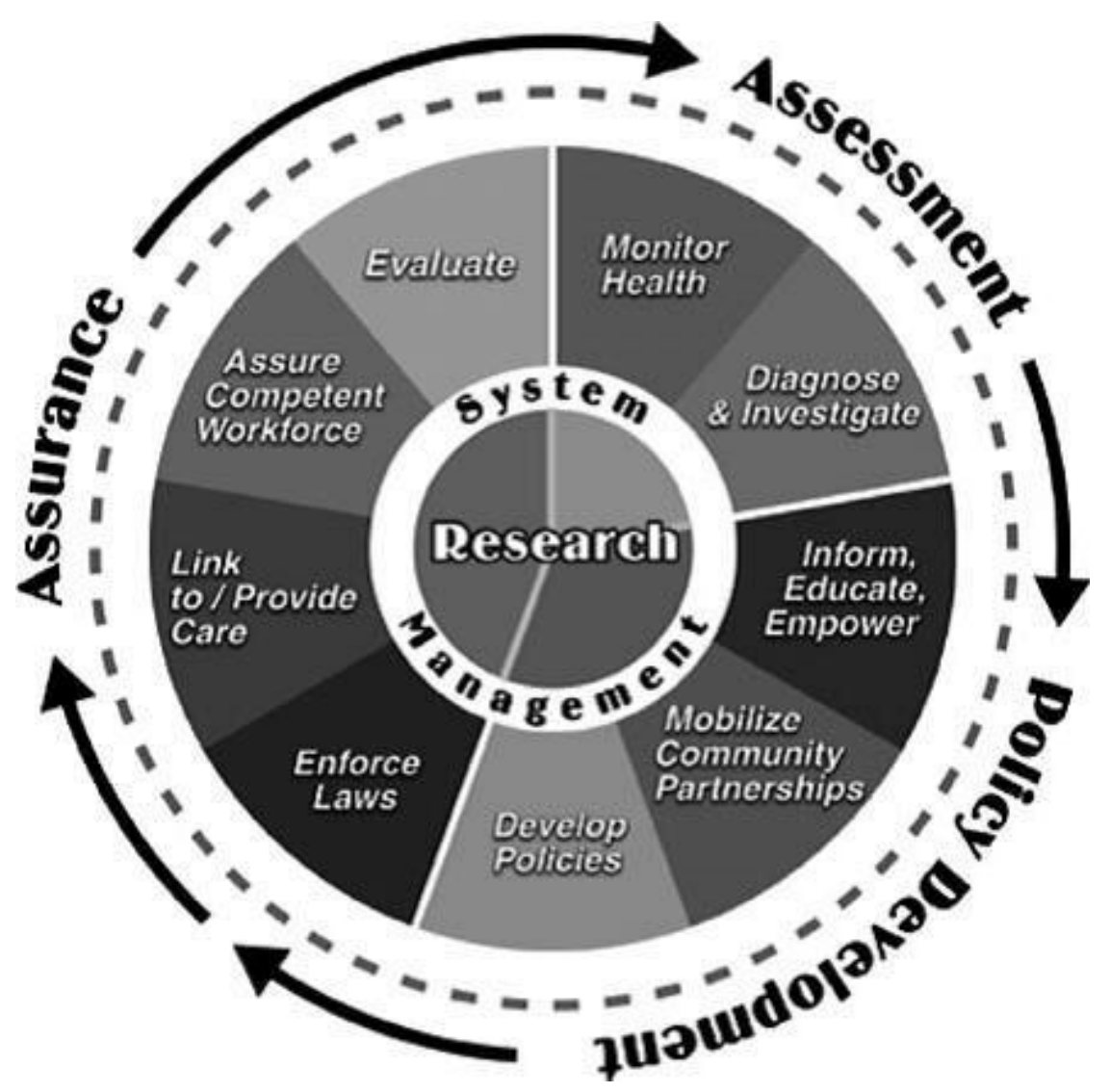

Figure 1: Public health trias/public health wheel (Institute of Medicine, 1988). 


\section{European Best Practice Guidelines for Quality Assurance, Provision and Use of Genome-based Information and Technologies}

1. Research

- Keep up with new insights from the basic sciences.

- Develop trans-disciplinary agendas for translational research.

- Promote sustainable funding for translational research to produce neutral and trustworthy information.

- Generate evidence to demonstrate when the use of GBIT in public health can improve health outcomes in a safe, effective and cost-effective manner.

- Engage community members and foster trust by supporting research collaborations and partnerships between academics and the public.

\section{Monitor Health}

- Develop prospective surveillance systems for personal health data that facilitate accurate and ongoing assessment of highly dynamic health information across the life course.

- Promote the use of personal files that contain comprehensive personal health information including 'omics' data and their interaction with all other health determinants.

- Ensure that data obtained from different sources and for different purposes can be securely linked.

- Maintain the locus of control for health information with the individual citizen.

- Support self-monitoring using ICT tools.

- Develop new strategies for the use of GBIT within the public domain.

- Maintain an infrastructure for the sharing of GBIT data in public health settings.

- Increase the use of Health Needs Assessment (HNA), HTA and Health Impact Assessment (HIA) tools for the evaluation of GBIT in public health.

\section{Diagnose and Investigate}

- Move from symptom- and phenotype-based approaches to pathway-based cloud diagnostics for the early identification of health problems in individual patients.

- Move from clinical utility to personal utility. 


\section{| CHAPTER VII}

- Identify which information is relevant to an individual, when, and for what purpose across the life course.

- Use GBIT to identify hazards to population health.

- Identify and assess new GBIT for proactive and timely decision-making.

- Ensure that the assessment of GBIT is comparative, comprehensive and constructive.

\section{Inform, Educate, Empower}

- Promote health literacy amongst all stakeholders: enable citizens (including health professionals), individually and cooperatively, to access, understand, appraise and apply information that will facilitate the application of GBIT for the benefit of individual citizens and their communities.

- Promote person-centered healthcare.

- Promote social media to enable proactive consumers ("prosumers") to manage their own health.

- Establish online platforms dedicated to informing users about validated GBIT as well as available related health services.

- Support participatory and personalized healthcare through the implementation of decision support tools that link the values and preferences of citizens to the best available evidence on GBIT.

- Ensure that potential users receive guidance on when and how to use public health assessment tools (HNA, HTA, HIA) applicable to GBIT.

\section{Mobilize Community Partnerships}

- Support the establishment and maintenance of national task forces (NTFs) on public health genomics. NTFs provide a platform for public health practitioners, basic scientists in genomics, HTA officials and policy makers to listen to, learn from and contribute to public health genomics, and to define the policy questions regarding GBIT as well as to assess GBIT at different stages in their life cycle.

- Promote public-private partnerships by establishing mechanisms, tools and models to link the needs of healthcare systems to those of the private sector and to foster the development of individualized technologies (e.g., learning adapting leveling model).

- Reinforce the key role of institutes of public health in community engagement. 
- Promote patient- and citizen-oriented social media activities as a new form of community partnership.

\section{Develop Polices}

- Reinforce the collaboration between PHGEN NTFs, national HTA agencies and parliamentary technology assessment groups to develop strategies to inform policy makers on the introduction and iterative assessment of GBIT in an effective, efficient and timely manner.

- Promote policies that cover all phases of the innovation pipeline by combining technology transfer activities with health policy processes: laboratory-enterprise, enterprise-regulation, regulation-reimbursement, and reimbursement-disinvestment.

- Improve the implementation of promising GBIT under conditions that safeguard the wellbeing of citizens.

- Support health policies related to GBIT based on good governance and trust.

- Treat GBIT as the most holistic approach to health information when developing health policies.

- Ensure that public health genomics works towards health in all policies.

- Support health policy making that takes into account the best available evidence, real-world context, resources and population characteristics as well as the needs, values and preferences of stakeholders.

- Translate knowledge in a timely manner from basic sciences into healthcare applications.

\section{Enforce Laws}

- Use the dynamics of GBIT as a unique opportunity to frame and enforce laws and regulations proactively.

- Ensure that GBIT and their use in public health meet applicable legal standards. In this regard, the privacy and security of personal health information should be safeguarded in accordance with applicable law.

- Work to correct perceptions of genetic exceptionalism and promote laws and regulations that address personal health information as a whole.

- Use modern ICT to protect personal health data. 


\section{| CHAPTER VII}

- Assess existing legal frameworks applicable to public health genomics on a regular basis and adapt them when necessary to ensure the consistency and visibility of enforceable rules on the use of GBIT in different contexts.

- Support implementation of the EU Data Protection Directive amongst European Member States to allow the use of personal health data for personal and public health purposes and to improve the outcome of health interventions.

\section{Link to/provide care}

- Reduce inequalities in health through sustainable access to and use of GBIT.

- Develop systems that support interoperability between personal and public health management.

- Support the integration of P4 medicine into healthcare provision.

\section{Ensure a Competent Workforce}

- Integrate GBIT into the professional training and lifelong learning curricula of health professionals.

\section{Evaluate}

- Establish and support a holistic and systems-based evaluation of the impact of GBIT, taking into account economic issues and the different European health systems.

- Promote a system in which technology transfer activities and policy-based public health assessment tools (HNA, HTA, HIA) run in parallel for the timely, effective and efficient evaluation of GBIT.

- Help and support the development of methods, information systems, tools and resources to adapt assessment tools to changing requirements. 


\section{Concluding Remarks}

The proposed list of guidelines under the Declaration of Rome is crucial for the implementation of GBIT amongst European Member States in order to improve public and personal health. Future PHGEN activities will continue to build on previous work to provide a platform for the developments indicated in these guidelines. We therefore strongly recommend that PHGEN activities continue to be supported on European, national and regional levels within the applicable healthcare framework.

\section{The next step: implementation of the 2012 Declaration of Rome in the EU Member States}

Because health is not just a value in itself but also a driver for growth, only a healthy population can achieve its full economic potential. As mentioned in the Europe 2020 agenda 'promoting good health is an integral part of the smart and inclusive growth objectives for Europe 2020. Keeping people healthy and active for longer has a positive impact on productivity and competitiveness. Innovation in healthcare helps take up the challenge of sustainability in the sector in the context of demographic change', and action to reduce inequalities in health is important to achieve 'inclusive growth'.

Thus, as the next step a Joint Action (JA) "Public Health Genomics and Personalized Healthcare: Implementing the 'European Best Practice Guidelines for Quality Assurance, Provision and Use of Genome-based Information and Technologies' in rare diseases and cancer" is planned being a joint financing of a public body or non-governmental organization by the European Community and one or more Member States. The planned JA fully supports and implements the Innovation Partnership Flagship Action of Active and Healthy Ageing. It covers not only Innovation/ Genomics and Health in All Policies (HiAP) in the current program "Together for Health" (2008 - 2013) but it also highly prepares for and contributes to the new program "Health for Growth " $(2014-2020)$ by applying innovative solutions for improving the quality, efficiency and sustainability of health systems, putting the emphasis on human capital and the exchange of European best practices. As such, the planned JA will help Member States, which are under pressure to strike the right balance between providing universal access to high-quality health services and respecting budgetary constraints, to reduce healthcare costs and substantially improve the quality of care to all citizens now and in the future. The European best practice guidelines developed within PHGEN II and summarized in the Declaration of Rome address many areas of the Health for Growth Program, such as HTA, medical devices, clinical trials and medicinal products, and 


\section{| CHAPTER VII}

strengthen the link between technological innovation and its effective and responsible uptake and commercialization.

Therefore, the planned JA will be a very timely and essential next step by:

- Supporting the Member States to prepare in time for the paradigm shift towards personalized healthcare.

- Bringing together European stakeholders with a common objective and commitment to reduce the burden of common complex and rare disorders, and providing Member States with a framework for sharing information, resources, best practices and expertise in monitoring, surveillance, knowledge integration, prevention and care.

- Helping in the identification of common action at an EU level that can provide added value to national efforts.

- Helping with the implementation of the HiAP approach and the concepts of HNA, HTA and HIA in supporting action for active and healthy ageing.

- Serving as an umbrella for researchers from the different stages of translational research in the life sciences ("from cell to society") and market authorization, as well as fostering this translational process to the policy making and public health level.

- Focusing on two key policy areas of application, rare diseases and cancer.

- Using the well-established 13 NTFs of PHGEN I as the core group and extending it to the maximum of Member States.

In conclusion, building on PHGEN I and II the planned JA will help address the transition to personalized healthcare and personal health from the data integration and modeling perspective points of view in all EU Member States, Applicant and FTA-EEA countries within the upcoming years. 
CHAPTER VIII

CONCLUSIONS PART B 


\section{Conclusion}

With regard to specific objectives of Part $B$, with respect to chapter $\mathbf{V}$, the thesis completed and answered the objectives pertaining namely:

- Demonstrate the complexity of biology reasoning for the move in the post-genomics era to systems biology and systems medicine for personalized health applications and beyond

In this chapter, it was demonstrated that molecular biology is quite complex and requires computational power to partially elucidate answers. However, this has given us more questions than answers. The chapter shows that molecular interactions are complex and form also a hierarchy of thousands of interactions. These interactions can lead to feed-back/forward control, modularity, redundancy as well as multitasking. Also structure of a molecule also affects several functions as well as localization. Further genetic switches exist in these networks. Several previous unclassified medical relations have come up due to the interactome. Several such complex interactions were described including interations with environmental factors. This explosion of data has only been possible with the explosion of 'omics' research through computational power. However, given this large amount of data, a more holistic approach is needed; this can be addressed through systems biology. With the advent of social media and the individual/citizen becoming aware of the uniqueness of individuals, the individual is demanding for a more personalized approach to healthcare. Only a systemic approach or holistic approach can make it possible to understand the complexity of biological data and address personalized healthcare applications.

\section{Take Home Message}

Complexity of biology has been partially elucidated through computational power in the form of genome-based technologies and systems biology. This has given a push towards personalized healthcare and within that personalized medicine.

The Learning-Adapting-Leveling model demonstrates its potential application in personalized healthcare through the EU flagship project ICT Future of Medicine. ICT Future of Medicine addresses the environmental aspects and the LearningAdapting-Leveling model is being implemented in the project to ensure by the time the technology is rolled out it is ready for healthcare implementation.

Chlamydia trachomatis is a leading cause of tubal pathology and infertility in women. Potential SME based diagnostic kit for genetic markers in tubal pathology and infertility to be used in conjunction with Chlamydia trachomatis serology is proposed to avoid misdiagnosis. The Learning-Adapting-Leveling model is proposed to be implemented to this potential SME based diagnostic kit.

EU Guidelines on the QA, provision and use of genome-based information and technologies have been endorsed by EU member states to compensate the currently limited scope of existing guidelines and the model has been incorporated into them. 
- Signifying the issue of taking into account environmental factors interacting with 'omics' factors as well as health systems when developing such applications

This chapter has emphasized that the development of technologies generally do not take into account the environmental factors, such as lifestyle, climate, social, etc. It is now known that these do affect the exposome having consequences to 'omics'. In order to implement the P4 medicine, technological development needs to take into account environmental factors like socio-economic, lifestyle and the molecular determinants of health which all affect molecular interactions. Also, healthcare systems need to be taken into account while developing the technology. The Learning-Adapting-Leveling model addresses the issue of healthcare systems as well.

- Using the EU flagship pilot project ICT Future of Medicine (ITFoM, www.itfom.eu) as example of European research project where the Learning-Adapting-Leveling model is being implemented

The ITFoM project does indeed take into account the environmental factors mentioned above, however it is still to be seen how it will be utilized. ITFoM aims to develop a virtual human on the computer, on which simulations like drug reactions and treatments can be done. Thereafter it can be transferred to the actual individual. The Learning-AdaptingLeveling model has been implemented in the ITFoM project to ensure that the required unseen assessment through the technology transfer pipeline namely HNA, HTA and HIA is being done to ensure that the technology conforms to the national and regional healthcare requirements. Also, the model is being used to assess throughout the development of the technology the value of information and to ensure the 10 essential public health tasks for integration is implemented. The Learning-Adapting-Leveling model is also being lobbied with relevant stakeholders. This will ensure that by the time the technology (here the simulation tool) is rolled out, it can be readily taken up by the healthcare system.

With regard to specific objectives of Part B, with respect to chapter VI, the thesis completed and answered the questions pertaining namely:

- Applying it to Chlamydia trachomatis as the leading cause in tubal pathology and infertility In this chapter Chlamydia trachomatis has been reviewed as the leading cause in tubal pathology. The chapter reviews all relevant data on chlamydia trachomatis and provides an overview of the current state of the art on host genetic markers in relation to infection and the subsequent late complications it can cause in susceptible people. The chapter proposes a new clinical diagnostic approach in subfertility diagnostics based on this overview. The potential translational and clinical value of adding diagnostic host genetic marker profiles relating to infection and inflammation was proposed to be added to the current diagnosis techniques which are in the case of subfertility triage in women, Chlamydia serology. Reason being there is significant amounts of misdiagnosis as several women without tubal pathology have to go through the invasive and expensive laparoscopy with the traditional Chlamydia trachomatis serology tests. 
- Demonstrating the implementation of the Learning-Adapting-Leveling model to potential SMEs Based on the above mentioned problem, it has been considered to develop for example a potential SME based diagnostic kit for genetic markers in tubal pathology and infertility to be used in conjunction with Chlamydia trachomatis serology. In order to realize this endeavor large scale SNP typing and SNP identification in parallel to cohorts is required. This is currently ongoing. It is hoped that this work will provide insight into what type of host genetic profile can help improve subfertility diagnoses and whether the added sensitivity, specificity, and positive and negative predictive value will realize the hope that carrier traits will significantly increase the ability to predict and identify those at greatest risk of severe complications from C. trachomatis infection. The LearningAdapting-Leveling model will be used and applied here as an overarching framework to answer that question. As the potential development of the SME and the identification of genetic markers are being evaluated, the Learning-Adapting-Leveling model will closely follow the whole process. Early on assessments can be evaluated starting with HNA through the technology transfer pipeline of this potential diagnostic kit. As a result it is hypothesized that by the time the diagnostic kit is rolled out it would have conformed to the healthcare requirements and can be readily taken up in daily practice through lobbying.

With regard to specific objectives of Part B, with respect to chapter VII, the thesis completed and answered the objectives pertaining namely:

- Contributing to the development of the European best practice guidelines for genome-based information and technologies (PHGEN II)

Adapted and shortened from:

Brand A, Lal J, Malats N, Gutierrez-Ibarluzea I.

The Public Health Genomics Network (PHGEN).

The Newsletter of the British Society of Human Genetics 2013, (48):53-54.

Genomics is a highly dynamic field and, as such, represents a moving target for public health. Public health is shifting from a focus on the population towards an emphasis of the individual as a means of supporting the wellbeing of the population. In particular, we are entering the era of predictive, personalized, preemptive and participatory (P4) medicine supported by advanced technological infrastructure. These changes represent a paradigm shift in our approach to healthcare and will go hand in hand with a major reclassification of disease. The challenge now is to understand how all of these changes will impact public health and how to ensure that they are translated effectively into benefits for individual citizens and society as a whole. Thus, there is a need to develop guidelines aiming not to close doors. Instead, the goal is to create a vision that allows for flexibility and adaptability in their implementation in order to have a maximum impact on health, the health care infrastructure, health technologies and economic growth in the health sector. Therefore, the European Commission asked to develop "European Best 
Practice Guidelines for Quality Assurance, Provision and Use of Genome-based Information and Technologies" to support the Member States (and other relevant stakeholders) to more efficiently and effectively work together at European level in addressing the challenges deriving from emerging genome-based information and technologies and to prepare for the paradigm shift of personalized healthcare in time. The implementation of the concept of public health genomics being the responsible and effective translation of genome-based knowledge and technologies for the benefit of population health requires modifications of public health and health governance systems on all levels. The Public Health Genomics European Network (PHGEN II) fulfills this task. It is an EU DG SANCO funded and European Agency for Health and Consumers (EAHC) issued project (EU Project No. 20081302, 2009-2012), which produced recently the first edition of "European Best Practice Guidelines for Quality Assurance, Provision and Use of Genome-based Information and Technologies". The guidelines will assist all EU Member States, Applicant and EFTA-EEA countries with evidence-based guidance on the timely and responsible integration of genome-based information and technologies into healthcare systems for the benefit of population health. They build on the extensive work of the Public Health Genomics European Network (PHGEN, www.phgen.eu) being the cornerstone in the development of public health genomics in Europe. In this concept, genome-based information is very holistic and includes not only all 'omics' data, but also environmental, socioeconomic, and lifestyle factors as well as information on health systems. The process of the guidelines development was in line with international standards and acknowledges the diversity and cultural differences in Europe. Key experts such as public health experts, EU lawyers, human geneticists, ethicists, systems biologists, Health Technology Assessment (HTA) experts, representatives from the private sector and patient groups as well as key policymakers had been involved in PHGEN II. On the 19th and 20th of April 2012, experts from across the field of public health genomics representing key European and national organizations and institutions from policymaking, academia and private sector as well as the representatives from all EU Member States came together at the final PHGEN II meeting in Rome to discuss the future of public health genomics and to endorse the Declaration of Rome on 19 April 2012, a summary of the "'European Best Practice Guidelines for Quality Assurance, Provision and Use of Genome-based Information and Technologies" (Declaration of Rome, www.phgen.eu). PHGEN II builds on the definitions that were developed and agreed in PHGEN I, such as the glossary on public health genomics and the status of genetic information and genetic testing. The definitions provided by PHGEN II take into account the most recent developments in the fields of genomics, systems biology, and systems medicine, which provide the evidence base for the "European Best Practice Guidelines for Quality Assurance, Provision and Use of Genome-based Information and Technologies". The terms genome-based information (GBI) and genome-based technologies (GBT) are encompassed by the term genome-based information and technologies (GBIT). The ultimate objective of PHGEN II is to enable informed decisions at the macro, meso and micro levels regarding quality assurance, provision and use of emerging GBIT. The means 
chosen by PHGEN II to achieve this is the preparation of European best practice guidelines to support this decision-making process now and in the future. However, meta level guidance is also needed. This meta level guidance can be achieved by ensuring that the 10 essential public health tasks, as described within the public health wheel or public health trias (assessment, policy development, assurance) can be adequately fulfilled in each jurisdiction on the basis of a common understanding of best practice guidelines for each task Within these best practice guidelines, translational research considerations are combined with system management under the concept of public health genomics.

- Integration of the Learning-Adapting-Leveling model into these guidelines

It can be seen from this chapter that the best practice guidelines is based around the 10 essential tasks of the public health wheel. This wheel synonymously called the public health genomics wheel is an essential part of the Learning-Adapting-Leveling model. Furthermore, the Learning-Adapting-Leveling model has been explicitly mentioned to be integrated in the best practice guidelines as demonstrated in this chapter. Therefore compatibility of the Learning-Adapting-Leveling model with the best practice guidelines is ensured. This also resolves the issue of currently existing guidelines which do not take into account genomics as a moving target and emerging technologies as previous guidelines mostly focused on clinical guidelines. 
Section 3

General Discussion 


\section{Discussion}

Through genome-based technologies and systems biology, personalized healthcare including personalized medicine has taken an exponential leap in its demand. For example, the market for personalized medicine is expected to reach $\$ 454$ billion by 2015 for the US market alone. However, depending upon the type of technology being implemented, a gap is noticed from time to market to daily practice of these genome-based technologies in healthcare systems. In other words, it is seen although a translation from the lab to industrial application (phase I) and also from the industrial application to market penetration (phase II), there seems to be an apparent delay in the uptake from the market to healthcare integration (phase III). By the time the delayed relevant technology is taken up in daily practice is can be considered less relevant as a more effective and efficient technology emerges onto the market. This plays out repetitively which was defined as the circle of destination in chapter II. As was also indicated, issues like lack of funding to research institutes and limited return of investments are hindrances which play a role. The latter however can be attributed to failure of the product and inaccessible markets or untimely interventions. The lack of funding is more of an inherent problem to the research institute and it's funders and does not compute with the delay in uptake of existing technologies on the market. Although the right timing of the introduction to market does play a role, but still even if this is properly addressed, the issue of timely uptake remains. Therefore these are not major reasons for the backlog.

This problem completely relates to the field of Public Health Genomics, which is defined as the responsible and effective translation of genome-based knowledge and technologies into public policy and health services for the benefit of population health. The sub-specialty of the field within public health to address the problem was elaborated and defined in chapter I as 'valorization in public health genomics'. It can be seen as the 'process of realization' of relevant added value 'bioproducts', i.e. technologies, in the domain of public health for benefit of the population and healthcare systems. Bioproducts are sustainable novel products or products satisfying novel health applications derived or inspired from genome-based information or technologies. Genome-based derives everything coming out of the human genome i.e. genomics, proteomics, metabolomics, etc. genome-based information includes the interactions with environmental factors such as lifestyle, climate, etc. Technologies can include a technique, process, methodology, tool, activity, diagnostic kit, application and technology in 
the general sense. There seems to be quite an overlap between the definition of 'bioproduct' and 'technology'. In essence the bioproduct is the technology developed.

\section{The Proposed Solution}

In this thesis the main reason for the bottleneck or backlog of effective and efficient real-time uptake of genome-based health applications was identified as the non-synergy and lack of communication between the academic-industrial complex and the decision makers of healthcare systems (as indicated in chapter I). The latter includes the agencies which assess the technologies and develop the recommendations for decision makers. This bottleneck also affects the return on investment mentioned partially due to making the market (in this case healthcare) time-wise inaccessible. Also, the academicindustrial complex mainly involved in the first 2 phases mentioned above use different tools than the healthcare systems (phase III). A cross-integration of different concepts (mainly in phase III) has been suggested to address translation from bench to healthcare.

Within the concept of valorization in public health genomics, in order to resolve this bottleneck, the Learning-Adapting-Leveling model (figure 1 below) was created bringing in for the first time the academic-industrial complex and decision makers of healthcare systems on the same platform moving together relatively parallel. Technology transfer is generally used with the first two phases mentioned in the paragraph above and chapter I. For the third phase mentioned above, the public health assessment tools are used. It is hypothesized that through the interactions between technology transfer and the public health assessment tools (HNA, HTA, HIA) through the development of the technology, academicindustrial complex will adapt their technology to conform to the healthcare needs taking into account the value of information and the public health genomics wheel as reference points. The process starts with basic research and development in the lab leading to an idea which can develop or address a need. As mentioned in chapters I and III, after some feasibility studies, the TT professionals approach the HNA professionals which in turn assess the developing technology based on needs and give feedback. The TT professionals go back to the drawing board and adapt the technology. Thereafter they approach the HTA professionals. The HTA professionals assess the technology as such and give feedback, with the TT professionals going back to the lab and adapt the technology. Lastly, the same process is addressed with HIA professionals. This can involve several feedback loops. 


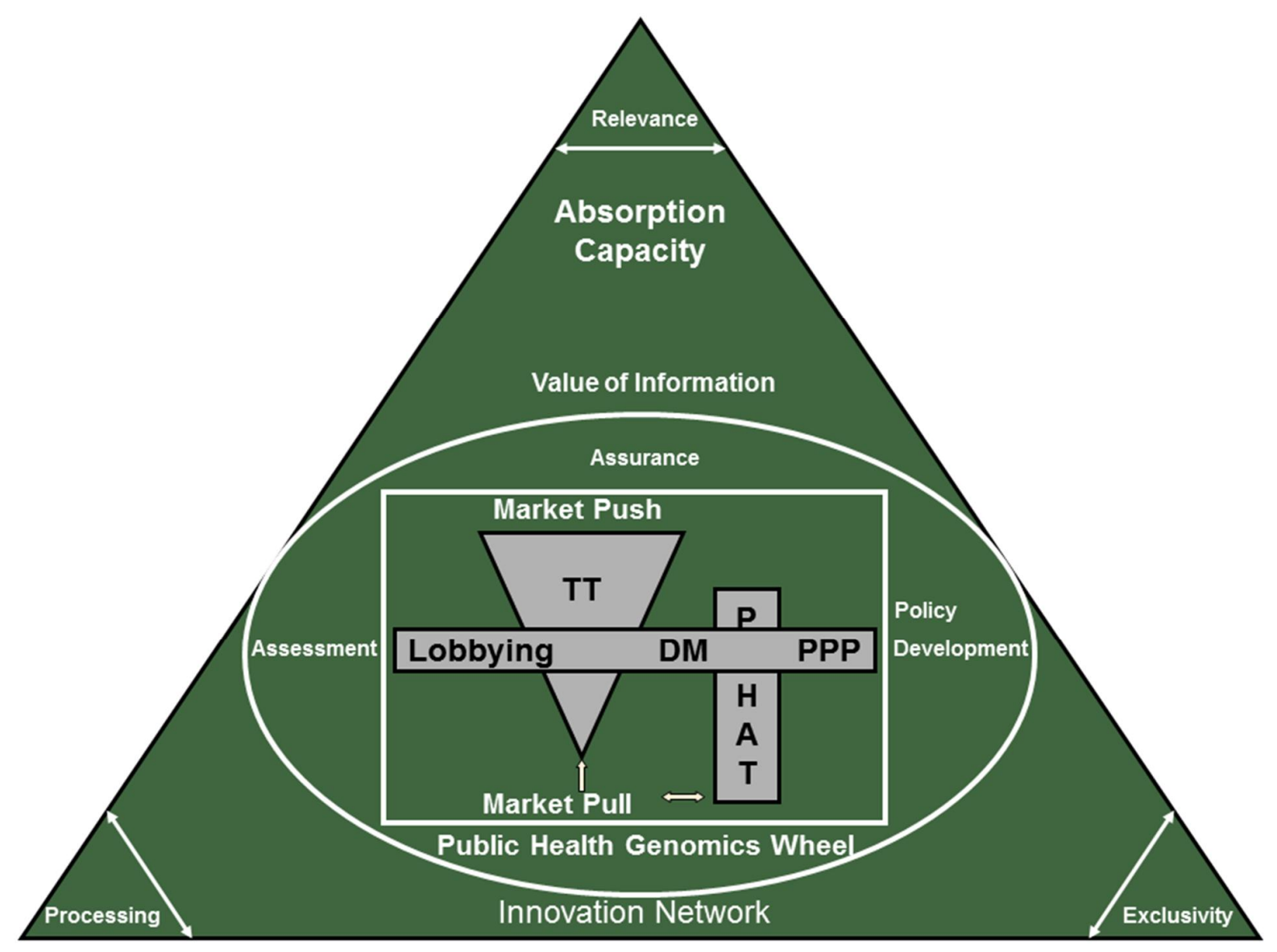

Figure 1: The Learning-Adapting-Leveling Model as the solution overarching framework.

During the whole process the value of information ( $\mathrm{Vol}$, as one of the two reference points) is seen from three perspectives, namely, the processing ability or understandability of the end user regarding the use of the technology, relevance to the target need and exclusivity (patents, etc.), which restrict its wide usage. These three perspectives are analyzed from two different fields of work. As indicated in chapter II, the value of information is analyzed from the business aspect as well as the HTA's Vol concept. This ensures that the technology covers business as well as public health aspects and some form of HTA requirements are fulfilled early on before feedback. On the other hand the public health genomics wheel defines the 10 essential tasks of public health needed to be addressed for successful integration of genome-based technologies into public health. These reference points are kept throughout the process when technology transfer professionals interact with the HNA, HTA and HIA professionals adapting and conforming their technologies to ensure no loop holes remain. 
As a result through feedback loops of interactions and improvements by the time the technology is ready to be rolled out it will have been conformed to the previously unseen requirements of policy. Consequently, it can be readily taken up by the relevant healthcare system and potential broadly defined public-private partnerships could be realized in the process. This also takes into consideration of lobbying of relevant technologies in the pipeline based on their assessments (by HNA, HTA and HIA) and conformity requirements addressed. The important part here being the early-on involvement of all stakeholders (patient groups, insurance, doctors, decision makers, etc.) through the interaction between the academic-industrial complex (through technology transfer) and the PHAT (HNA, HTA and HIA professionals). It is presumed logical that through this interaction other stakeholders will be eventually consulted early-on as the conformity analysis issue does take these stakeholders into account based on the need.

Initiatives like early HTA aiming to facilitate pre-market assessment of upcoming new technologies have been introduced. However they lack the technology transfer aspect of the academic-industrial complex. It is well known that modern HTA constitutes horizon scanning and/or constructive technology assessment for early on assessment of dynamically moving technologies. However, the true sense of these tools is not realized. Reason being that horizon scanning and constructive technology assessment can only assess early on or in real-time only those upcoming technologies whose knowledge is in the public domain or recently introduced on the market. Through the Learning-Adapting-Leveling model, the interaction between the academic-industrial complex and the HTA professionals early on in the development of the technology, a true sense of horizon scanning and/or constructive technology assessment can be realized as the early on evaluation will comprise of assessment through the pipeline of the technology development prior to its official release to the market or public domain. This also ensures that relevant technologies are not missed out as the technology developers will be approaching the HTA professionals.

As an outcome of this interaction through the innovation network in chapter I of this thesis, it has been stated that the progress and development can lead to the advantage of the industry to come to an earlyon decision on the technology being developed, whether to conform it further if feasible, or kill it entirely, thus saving on resources like time and money. Also, they could still continue with the technology not conformed, targeting the traditional market. On the other hand, mainly mentioned in chapters II and III, this can also possibly help decision makers to timely diffuse relevant technologies into 


\section{| GENERAL DISCUSSION}

health care and develop related policy-wise guidelines. One of the possible outcomes is that this can reduce the burden of disease as when the technology reaches the patient on time, it can help in a more effective and efficient manner to diagnose or treat the disease and possibly prevent it early on. Economically, this will seem more advantageous, therefore delayed integration of relevant technologies is indeed a problem.

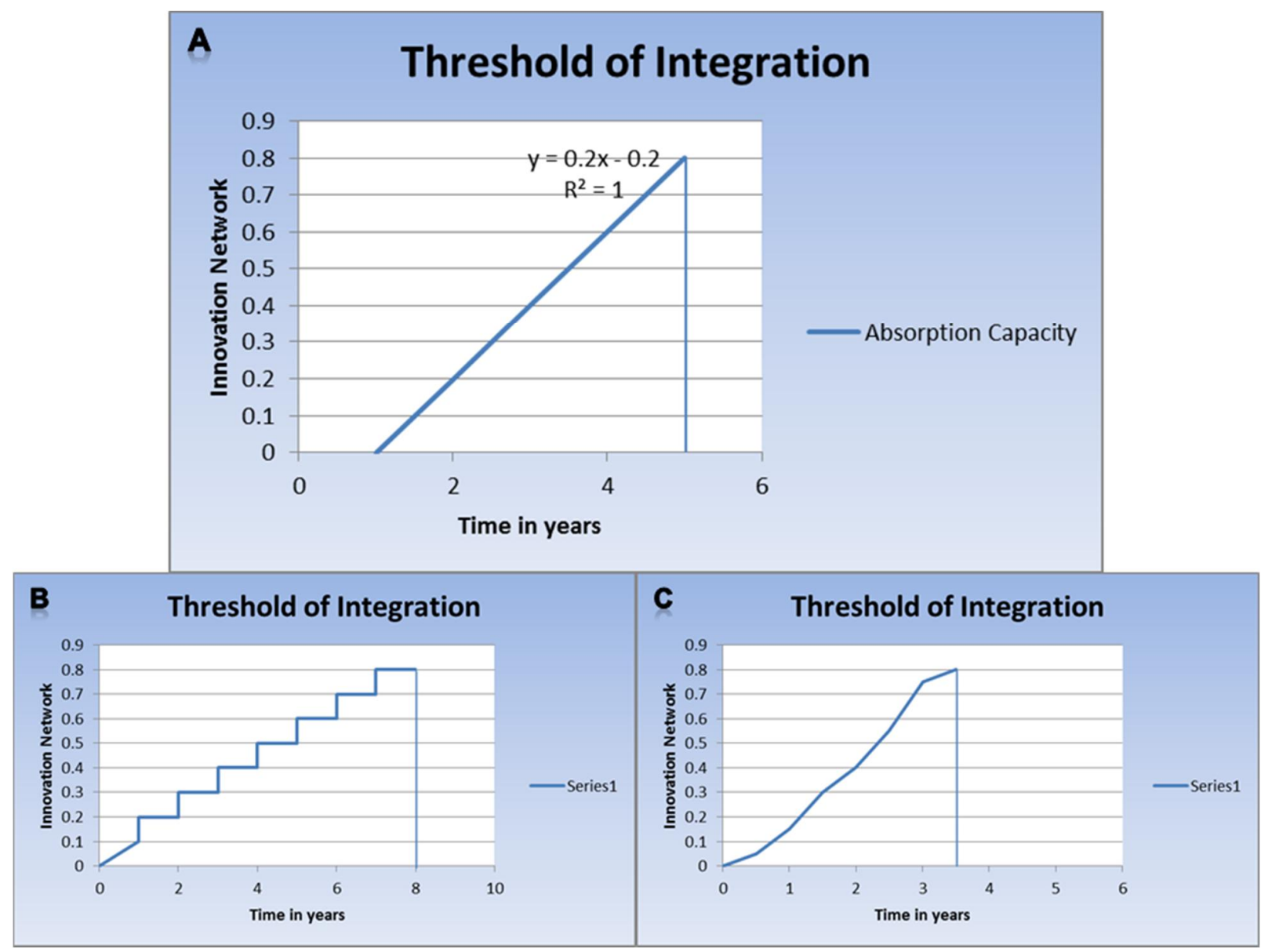

Figure 2: The absorption capacity as a function of the innovation network through time.

In chapter I, it has been stated that the higher the absorption capacity, the higher the possible success of this proposed model. In other words, the more cases or technologies which go through these interactions in the innovation network eventually will lead to broadly defined public-private partnerships for future cases. Each time a new technology goes through the now existing partnership/network, through experience, the better the interactions will be optimized in terms of efficiency and effectiveness. It can then be said the more optimized the interaction, the better the chance of success both in time and resources. This absorption capacity (see figure 2 above on threshold capacity) will never be $100 \%$ as 
there will be always room for improvement and unforeseen issues. Also the timeline of integration is dependent on the type of technology being developed and where. For example, pharmaceuticals can take more time than general technologies. In chapter I we have given an example on how this progressed and in chapter III, we elaborate on this developing the step-by step methodology of the framework as stated previously above.

\section{Coverage of the Model}

In chapter II, based on the definition of technology transfer it was proven that technology transfer covers all aspects of translational research from the lab till the market and return on investments. This includes technology transfer's coverage (see figure 3 ) of the concepts of valorization, spin-offs, regional valorization systems, drug discovery and open innovations, although can still be considered subjective. Given that technology transfer covers the complete spectrum from the lab to bringing the technology onto the market including return on investment, and since is it widely used in a variety of forms, it is the best representation of the academic-industrial complex. Therefore, it was considered one of the two core components of the Learning-Adapting-Leveling model.

\section{Translational Research}

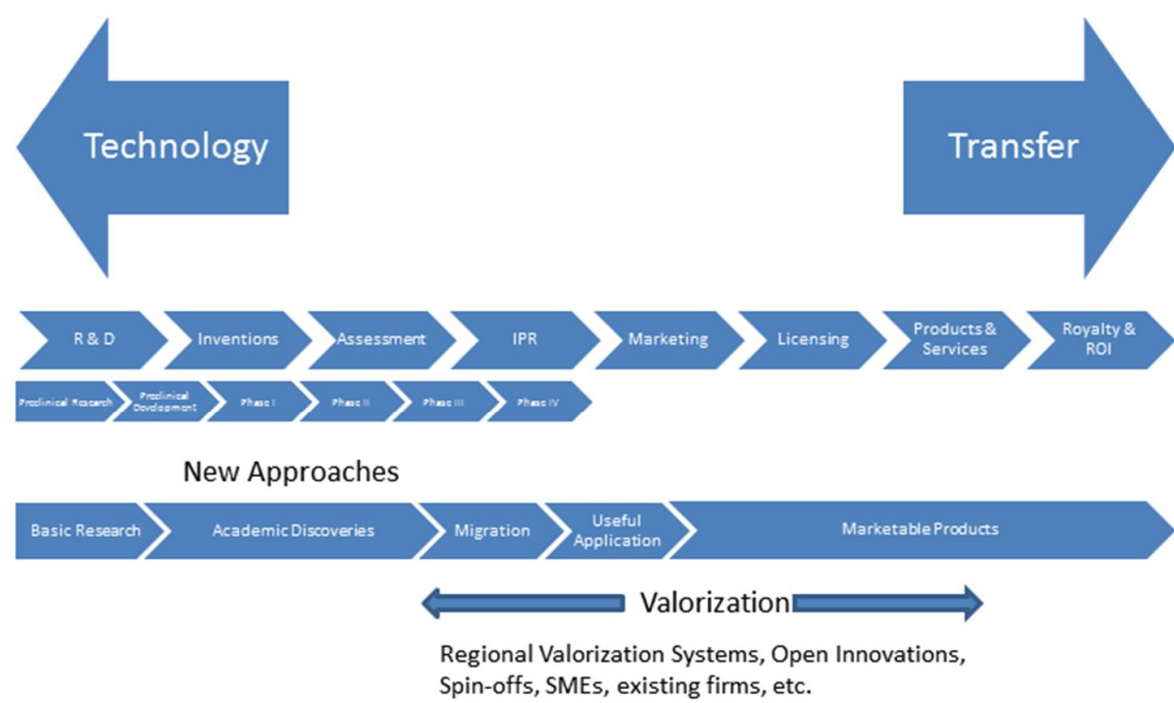

Figure 3: Technology Transfer (TT) as the overarching framework from bench to market. The third row indicates a subjective vew on the $T$ process, the fourth row is the drug discovery pipeline. The last two rows indicate the ovearching framework of TT. 


\section{| GENERAL DISCUSSION}

Similarly, for HNA, HTA and HIA were considered well defined popular methodologies important for decision making. Thus, their usage in the model as the public health assessment tools was the second core of the Learning-Adapting-Leveling model. The model considered to keep out certain other tools like ACCE, EGAPP, T1-T4 and PHG enterprise due to their limited scope and/or overlaps with the public health assessment tools as was demonstrated in chapter II. Importantly, the public health genomics wheel or the public health wheel was considered quite important for its inclusion in the model as a reference point. In the chapter it was demonstrated based on the definitions of the rejected tools with comparison with the definitions of the components of the public health genomics wheel, the latter covered all aspects of the rejected tools ensuring that possible gaps can be avoided and that extensive coverage is ensured. Furthermore, it was stated that the public health assessment tools (HNA, HTA and HIA) are also covered in a way over the public health genomics wheel; this being due to the reason that HNA, HTA and HIA are methods used to fulfill or to do some of the ten essential public health tasks of the wheel. However, the wheel itself was not considered the core component rather a reference point due to the fact that the public health genomics wheel defines the 10 essential tasks of public health which have to be considered for successful integration of genome-based technologies into public health and healthcare without a defined method. On the other hand the public health assessment tools in the form of HNA, HTA and HIA are well defined and proven popular methodologies referred to by decision makers for recommendations. In addition to the above the model also takes into consideration the value of information as the other reference point. As a consequence the Learning-Adapting-Leveling model is quite comprehensive in its approach and coverage of relevant tools for translation and implementation from the lab through the market to the implementation in healthcare. Therefore it can be considered an overarching framework.

As mentioned in chapter II, the coverage of the Learning-Adapting-Leveling model can also be considered addressing also the concept of 'diffusions of innovations' however the scope of the model is to utilize and incorporate it as opposed to studying the concept. In the Learning-Adapting-Leveling model's innovation network, these interactions amongst the technology transfer professionals and the HNA, HTA and HIA professionals including early on involvement of all stakeholders, address the four main components that impact a concept according to the concept of 'diffusion of innovations' namely, innovation, communication channels, time and the social system are addressed. The 'innovation' is addressed through technology transfer and its interactions with the public health assessment tools (HNA, HTA and HIA) regarding its spread. 'Communication channels' are addressed and established 
through the interactions within the model through the innovation network by the development of broadly defined public-private partnerships both formal and informal. 'Time' in the form of the bottleneck of effective and efficient real-time integration is the basis from which the LAL model was developed. 'Social system' is incorporated through these interactions in the innovation network with technology transfer conforming to the requirements of public health and health care systems via these assessments. Also the involvement of all stakeholders including patient groups, insurance companies, doctors, academicians, decision makers, HTA professionals, industry among others throughout the process of the model ensures that the 'social system' the Learning-Adapting-Leveling model works within is addressed. Furthermore, the ethical, legal, social and economic implications are addressed through tools in the public health assessment tools.

In addition, human capital is an important aspect of the model as it is according to the concept of 'diffusion of innovations'. Also, the categories of adopters (from diffusion of innovations), namely innovators, early adopters, early majority, late majority and laggards are encompassed within the Learning-Adapting-Leveling model. The motive in the model is to shift towards early adopters through the conformity requirements and stakeholder involvement during the implementation phase of an innovation early on. In addition, the concept of 'critical mass' in 'diffusions of innovations' can draw parallel with the 'absorption capacity' of the Learning-Adapting-Leveling model. Using the concept of 'diffusion of innovations', the LAL model can develop the required interactions for effective and efficient real-time uptake of genome-based technologies within its innovation network. In other words, even if the actors utilizing the model are not familiar with 'diffusion of innovations' they are bound to use those concepts knowingly or unknowingly as a result of the complex interactions of stakeholders within the model as concepts like social, change, mentality and organizational issues will be addressed in the model. On the other hand however, although adaptions since the original concept of diffusion of innovations (in 1990) has been done, it was the rationale behind the idea of the need of a systematic approach like HTA which is incorporated as one of the core components of the Learning-AdaptingLeveling model.

\section{The Model in Action}

Chapter $\mathbf{V}$ demonstrates that the Learning-Adapting-Leveling model is being piloted in the EU flagship pilot project ICT Future of Medicine (ITFoM). ITFoM aims to develop a virtual patient of a human taking all information from molecular to family history among others into account. The idea is that doctors, 


\section{| GENERAL DISCUSSION}

patients and healthy individuals can simulate the reaction to a drug for example before using it on the patient. Although ITFoM covers the environmental factors of genome-based information and technologies and all possible data, healthcare integration will be a bottleneck. Here the LearningAdapting-Leveling model is being used to ensure all needed interactions and assessments of HNA, HTA and HIA keeping in mind the two reference frames namely value of information and the public health genomics wheel are accomplished through the technology transfer pipeline. As a result, once the ITFoM technology being a simulation tool is ready to be rolled out into the market, given the conformity issues addressed and through the developing public-private partnerships as well as the currently ongoing lobbying of the relevance of the technological development, it in principle should be ready for uptake in real time by healthcare systems.

In chapter VI, it was described the significant contribution of Chlamydia trachomatis to tubal pathology and infertility. Here again the ongoing application of the Learning-Adapting-Leveling model is described. Generally, there is a misdiagnosis in the currently available diagnostic test known as the Chlamydia Antibody Test (CAT). The chapter builds the case for adding host genetic marker profiles as a supplement to the currently existing CAT. Through the possibility of a new spin-off company (TubaScan Ltd.) the aim is to develop a diagnostic kit based around this principle by currently working on large scale single nucleotide polymorphism typing and identification. This most likely will increase the sensitivity, specificity and positive \& negative predictive value of diagnosis of tubal pathology in women with subfertility such that clinical utility can be defined properly. The Learning-Adapting-Leveling model is planned to be applied as an overarching framework within this project through the development of the diagnostic kit. This will ensure that health policy aspects are covered early on through the process. Using the framework of interactions between technology transfer and HNA, HTA and HIA, it is aimed to ensure real-time uptake by the time the technology has been developed. Here again the true sense of horizon scanning or constructive technology assessment as early stated can be realized.

As stated in chapter VII, the Learning-Adapting Leveling model has been incorporated into the EU DG SANCO "European Best Practice Guidelines for Quality Assurance, Provision and Use of Genome-based Information and Technologies" to be used by all EU member states i.e. by the key stakeholders on European and national level. These guidelines have been developed around the 10 essential tasks of the public health genomics wheel (see figure 4), which form an essential part of the Learning-AdaptingLeveling model reference frame, therefore again shows the importance of the wheel. The Learning- 
Adapting-Leveling model has been explicitly mentioned in these European best practice guidelines. The final aim of the guidelines was to inform decision makers at the macro, meso and micro-levels. The guidelines are more of a guideline for guidelines. The proposed list of guidelines can be considered crucial for the implementation of genome-based information and technologies amongst European Member States in order to improve public and personal health. Based on the current guidelines, as the next step, a DG SANCO Joint Action is planned on 'public health genomics and personalized healthcare: implementing the European best practice guidelines for quality assurance, provision and use of genomebased information and technologies in rare diseases and cancer'. The planned JA fully supports and implements the Innovation Partnership Flagship Action of Active and Healthy Ageing. It covers not only Innovation/Genomics and Health in All Policies (HiAP) in the current program "Together for Health" (2008 - 2013), but it also highly prepares for and contributes to the new program "Health for Growth" (2014 - 2020) by applying innovative solutions for improving the quality, efficiency and sustainability of health systems, putting the emphasis on human capital and the exchange of European best practices.

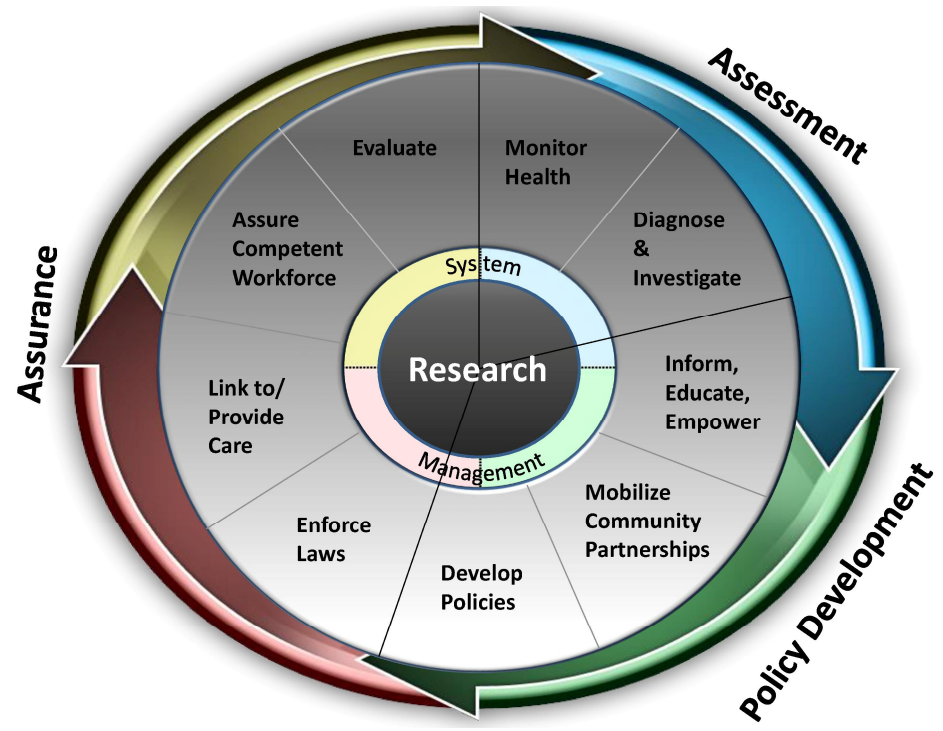

Figure 4: The 10 essential tasks of the PHG Wheel distributed over the 3 domains of assessment, policy development and assurance.

Coming back to these guidelines as given in chapter VII, apart from the model being incorporated in the specific sub-category of the category 'Mobilize community partnerships', the model due to its involvement with HNA, HTA and HIA including P4 medicine, as well as the guidelines developing the basis from the public health genomics wheel, ensures that the model is covered through all the guidelines mentioned. Examples of which are given below from the developed guidelines of the 10 categories (coverage of the model given in italics) 


\section{| GENERAL DISCUSSION}

\section{Research}

- $\quad$ Keep up with new insights from the basic sciences

The Learning-Adapting-Leveling model revolves around the concept of true horizon scanning and constructive technology assessment through early on involvement of these agencies (HNA, HTA and HIA) with technology transfer professionals or basic scientists working in applied research. Such basic scientists can be considered as entrepreneurs generating spinoffs as a result. Alternately they can just be applied researchers with potential applications another picks up for commercialization. This can be realized through the public-private partnerships of the model.

- Develop trans-disciplinary agendas for translational research.

The Learning-Adapting-Leveling model is trans-disciplinary as it involves a lot of actors from basic scientists, systems biologists, omics scientists (academia), industry, health research and policy through HNA, HTA and HIA, government through decision making, regulatory bodies, regional administration, medicine through doctors, patients and health professionals, insurance and reimbursements, etc. Again through the public-private partnerships in the innovation network of the model these agenda for translational research can be set.

- $\quad$ Promote sustainable funding for translational research to produce neutral and trustworthy information.

The recent EU DG SANCO call (tender) of a half a million Euros on dialogue between HTA professionals and technology developers indicates to answer this guideline (the tender of which completely is the Learning-Adapting-Leveling model). Future grants seem to be likely.

- Generate evidence to demonstrate when the use of GBIT in public health can improve health outcomes in a safe, effective and cost-effective manner.

The interactions between the academic-industrial complex and the public health assessment tools resulting in adaptions to technological conformity and the lobbying based on generated evidence is within the framework of the model. The technology transfer professionals have to demonstrate the evidence of the benefit of GBITs (their developing technologies) to healthcare in the Learning-Adapting-Leveling model.

- Engage community members and foster trust by supporting research collaborations and partnerships between academics and the public.

Early on involvement of all stakeholders and public-private partnerships are a basic principle in the Learning-Adapting-Leveling model, therefore this is taken into consideration. 


\section{Monitor Health}

- $\quad$ Develop prospective surveillance systems for personal health data that facilitate accurate and ongoing assessment of highly dynamic health information across the life course.

- $\quad$ Promote the use of personal files that contain comprehensive personal health information including 'omics' data and their interaction with all other health determinants.

The above two fall within the perspective of personalized healthcare. The model was developed with omics research (combined with its interactions with environmental factors) and systems biology in mind giving the output into personalized healthcare. The basic principle of monitor health falls within the reference frame of the PHG wheel of the LearningAdapting-Leveling model.

- Ensure that data obtained from different sources and for different purposes can be securely linked.

This brings in the ELSI (ethical, legal, social implications) model of HTA within the LAL model (e.g. data protection) as well as HIA. Again this falls within our reference frame.

- $\quad$ Maintain the locus of control for health information with the individual citizen.

- $\quad$ Support self-monitoring using ICT tools.

The above two fall in the category of personalized healthcare and personalized medicine on which the model was developed.

- $\quad$ Develop new strategies for the use of GBIT within the public domain.

- $\quad$ Maintain an infrastructure for the sharing of GBIT data in public health settings.

The above two fall again within the promotion or execution of the model through the interactions in the innovation network.

- $\quad$ Increase the use of Health Needs Assessment (HNA), HTA and Health Impact Assessment $(\mathrm{HIA})$ tools for the evaluation of GBIT in public health.

This becomes quite self-explanatory in the Learning-Adapting-Leveling model being the core components.

\section{Diagnose and Investigate}

- $\quad$ Move from symptom- and phenotype-based approaches to pathway-based cloud diagnostics for the early identification of health problems in individual patients.

- Move from clinical utility to personal utility. 


\section{| GENERAL DISCUSSION}

The above two again fall in the category of omics research (with its interactions with environmental factors) and systems biology (giving way towards personalized healthcare) which are the tools being translated into public health through the model.

- Identify which information is relevant to an individual, when, and for what purpose across the life course.

This falls for example, within the Value of Information reference frame of the LearningAdapting-Leveling model.

- Use GBIT to identify hazards to population health.

- Identify and assess new GBIT for proactive and timely decision-making.

- Ensure that the assessment of GBIT is comparative, comprehensive and constructive.

- Again the above three are quite self-explanatory given the scope of the developed model (Learning-Adapting-Leveling) using HNA, HTA and HIA through the technology transfer pipeline for true horizon scanning and constructive technology assessment.

\section{Inform, Educate, Empower}

- Promote health literacy amongst all stakeholders: enable citizens (including health professionals), individually and cooperatively, to access, understand, appraise and apply information that will facilitate the application of GBIT for the benefit of individual citizens and their communities.

- Promote person-centered healthcare.

- Promote social media to enable proactive consumers ("prosumers") to manage their own health.

- Establish online platforms dedicated to informing users about validated GBIT as well as available related health services.

- Support participatory and personalized healthcare through the implementation of decision support tools that link the values and preferences of citizens to the best available evidence on GBIT.

Here time and again, the above mentioned six points fall within the core principle of the Learning-Adapting-Leveling model's "early-on" involvement of all stakeholders through the innovation network.

- Ensure that potential users receive guidance on when and how to use public health assessment tools (HNA, HTA, HIA) applicable to GBIT. 
This comes through the iterations of the feedbacks on using the defined methodology of the Learning-Adapting-Leveling model as given in chapter III.

\section{Mobilize Community Partnerships}

- Support the establishment and maintenance of national task forces (NTFs) on public health genomics. NTFs provide a platform for public health practitioners, basic scientists in genomics, HTA officials and policy makers to listen to, learn from and contribute to public health genomics, and to define the policy questions regarding GBIT as well as to assess GBIT at different stages in their life cycle.

- Promote public-private partnerships by establishing mechanisms, tools and models to link the needs of healthcare systems to those of the private sector and to foster the development of individualized technologies (e.g., learning adapting leveling model).

The above two mentioned guidelines again fall within the broadly defined concept of publicprivate partnership as mentioned in the Learning-Adapting-Leveling model.

- Reinforce the key role of institutes of public health in community engagement.

- $\quad$ Promote patient- and citizen-oriented social media activities as a new form of community partnership.

The above two guidelines apart from being part of the public-private partnerships as mentioned in the Learning-Adapting-Leveling model also involves the model's core principle of early-on involvement.

\section{Develop Polices}

- $\quad$ Reinforce the collaboration between PHGEN NTFs, national HTA agencies and parliamentary technology assessment groups to develop strategies to inform policy makers on the introduction and iterative assessment of GBIT in an effective, efficient and timely manner. This falls within the public-private partnership category of the model.

- Promote policies that cover all phases of the innovation pipeline by combining technology transfer activities with health policy processes: laboratory-enterprise, enterprise-regulation, regulation-reimbursement, and reimbursement-disinvestment.

In chapter I, it was stated that government driven regulatory requirements would greatly benefit the execution of the model, this becomes apparent with this statement as well as 


\section{| GENERAL DISCUSSION}

given current EU calls of which the model answers (including the EU tender previously mentioned).

- Improve the implementation of promising GBIT under conditions that safeguard the wellbeing of citizens.

The ELSI of HTA and HNA assessment as well as the public-private partnerships answer this guideline within the model.

- Support health policies related to GBIT based on good governance and trust.

The government driven regulatory requirements are favorable to the model.

- $\quad$ Treat GBIT as the most holistic approach to health information when developing health policies.

This guideline is what the model wishes to translate.

- Ensure that public health genomics works towards health in all policies.

This again answers the government driven regulatory requirements

- Support health policy making that takes into account the best available evidence, real-world context, resources and population characteristics as well as the needs, values and preferences of stakeholders.

This is answered by HNA, value of information as well as early on involvement of all stakeholders as mentioned in the model.

- $\quad$ Translate knowledge in a timely manner from basic sciences into healthcare applications.

The final objective of the Learning-Adapting-Leveling model.

\section{Enforce Laws}

- Use the dynamics of GBIT as a unique opportunity to frame and enforce laws and regulations proactively.

This guideline works well with the model as the model also aims to develop policies on GBITs through the interactions between the academic-industrial complex and the decision makers through the public health assessment tools (HNA, HTA and HIA).

- Ensure that GBIT and their use in public health meet applicable legal standards. In this regard, the privacy and security of personal health information should be safeguarded in accordance with applicable law.

This falls within the category of ELSI of the model. 
- Work to correct perceptions of genetic exceptionalism and promote laws and regulations that address personal health information as a whole.

- Use modern ICT to protect personal health data. Data protection, health literacy (reference frame) and personalized healthcare are addressed through this thus alleviating the model.

- $\quad$ Assess existing legal frameworks applicable to public health genomics on a regular basis and adapt them when necessary to ensure the consistency and visibility of enforceable rules on the use of GBIT in different contexts.

The constant adaptions and iterations in the Learning-Adapting-Leveling model will also promote the upgrade or revisions in health policies and legal frameworks to accommodate the requirement of GBITs by the interactions in the innovation network including its consequential lobbying.

- Support implementation of the EU Data Protection Directive amongst European Member States to allow the use of personal health data for personal and public health purposes and to improve the outcome of health interventions.

The statement of this guidelines again works towards the positive usage of the model.

\section{Link to/provide care}

- $\quad$ Reduce inequalities in health through sustainable access to and use of GBIT.

- Develop systems that support interoperability between personal and public health management.

The above two mentioned guidelines are addressed through the interactions of the LearningAdapting-Leveling model developed potentially into public-private partnerships. As a consequence, timely interventions can reach the patient reducing inequalities and management can be made consistent over time.

- Support the integration of P4 medicine into healthcare provision. The Learning-Adapting-Leveling model also addresses $P 4$ medicine.

\section{Ensure a Competent Workforce}

- Integrate GBIT into the professional training and lifelong learning curricula of health professionals. 


\section{| GENERAL DISCUSSION}

This involves the 'processing ability' or understandability, one of the three components of the value of information reference frame in the model. In addition, the PHG wheel reference frame's 'educate' covers this as well. This can be achieved through lobbying.

\section{Evaluate}

- Establish and support a holistic and systems-based evaluation of the impact of GBIT, taking into account economic issues and the different European health systems.

This directly relates to HIA of the model as well as partly HTA.

- Promote a system in which technology transfer activities and policy-based public health assessment tools (HNA, HTA, HIA) run in parallel for the timely, effective and efficient evaluation of GBIT.

This is the government driven regulatory requirement which supports the functioning of the model.

The Learning-Adapting-Leveling model has been lobbied through self-citations, other citations, high level policy reports, grants and presentations internationally. Below the list as a result of this lobbying and promotion of the model is given:

\section{Related Publications (citing the original)}

- Brand A, Lal JA. European Best Practice Guidelines for Quality Assurance, Provision and Use of Genome-based Information and Technologies: the 2012 declaration of Rome. Drug Metabolism and Drug Interactions 2012, 27(3):177-182.

- Lal JA. Addressing the backlog of genome-based technologies in healthcare. The Newsletter of the British Society for Human Genetics 2013, 48:59-60.

- Lal JA, Malogajski J, Verweij SP, de Boer P, Ambrosino E, Brand A, Ouburg S, Morré SA. Chlamydia trachomatis Infections and Subfertility: Opportunities to Translate Host Pathogen Genomic Data into Public Health. Public Health Genomics 2013, 16:50-61.

- Lal JA, Sudbrak R, Lehrach H, Brand A. Functional Dynamics: From Biological Complexity to Translation and Impact in Healthcare Systems. Journal of Computer Science and Systems Biology 2013, 6(2): 088-092.

- Brand A. Personalisierte Medizin: Von der Vision zur Realität. Bulletin SAMW 2012, 3112:2-4. 
- Brand A, Morré, SA. Public Health Genomics - public health goes personalized. Tijdschrift voor Gezondheidswetenschappen 2013, 91(2):86-87.

- Brand A. Public Health Genomics and Personalized Healthcare: a pipeline from cell to society. Drug Metabolism and Drug Interactions 2012, 27(3):121-123.

- Brand A. Public Health Genomics - from cell to society. The Newsletter of the British Society for Human Genetics 2013, 48:51-52.

- Lal JA, Vaidya A, Gutiérrez-Ibarluzea I, Dauben HP, Brand A. The LAL Model: from theory to hypothesis of steps for implementation of basic genome-based evidences in personalized medicine. Personalized Medicine 2013, 10(7):683-701.

- Lal JA, Morré SA, Brand A: The overarching framework of translational and integration: a case for the LAL model. Personalized Medicine 2013, In Press.

\section{Other articles/publications citing the original article}

- Malogajski J, Brankovic I, Verweij SP, Ambrosino E, van Agtmael MA, Brand A, Oudurg S, Morré SA. Translational Potential into Health Care of Basic Genomic and Genetic Findings for Human Immunodeficiency Virus, Chlamydia trachomatis, and Human Papilloma Virus. Biomedical Research International 2013, 2013:1-10

- Lotte S, van de Wetering G, Groothuis-Oudshoorn K, Retel V. A Systematic and Critical Review of the Evolving Methods and Applications of Value of Information in Academia and Practice. Pharmacoeconomics 2013, 31(1):25-48.

- Joly Y, Dove ES. Moving Beyond Commercialization: Strategies to Maximize the Economic and Social Impact of Genomics Research. Policy Brief No. 5, Genome Canada April 2012 pages 1-13.

- Anzenbacher P, Nekvindová J. Perzonalizovaná medicína - současná praxe a př́sliby do budoucna. Klinická farmakologie a farmacie2012, 26(3):123-125

- Sanders MS. Host genetic variants in susceptibility, severity and outcome of bacterial meningitis. Chapter 9, Doctoral Thesis - Medicine. Vrije Universiteit Amsterdam. 27-02-2013.

\section{High level policy reports incorporating the model}

- European Science Foundation Forward Look: Personalized Medicine for the European citizen. Towards more precise medicine for the diagnosis, treatment and prevention of disease (iPM), 2012, pp. 17, 41. 


\section{| GENERAL DISCUSSION}

- European Alliance for Personalized Medicine: Innovation and Patient access to personalized medicine. Report from Irish Presidency Conference March 20th/21st 2013.

- ICT Future of Medicine Pilot Flagship Final Report. 30 April 2012, Pages 25, 26, 69, 70, 76.

- Das war die Amgen Science Lounge 2013. AMGEN.

\section{Related published conference proceedings}

- Lal J. Valorization of genome-based technologies in public health. In proceedings of the 4th European Public Health Conference. European Journal of Public Health 2011, Vol. 21, Supplement 1. 10 November, Copenhagen.

- Vondeling H, Lal J, Douw Karla, Cassiman JJ, Brand A. Public Health Genomics and Health Technology Assessment. In proceeds of the 9th HTAi Annual Meeting. Gac Sanit. 2012, 26(Espec Congr 2):6.

- Lal JA. Valorization of Genomics in Public Health. Biomedica Life Science Summit 2011, Eindhoven Netherlands.

- Lal JA, Schulte in den Bäumen T, Morré SA, Brand A. Valorizing Genomics in Public Health. Genetica Retraite 2011, Kerkrade Netherlands.

\section{Grants incorporating the model}

- EU Framework Program 7 (FP7)

- COST - European Cooperation in the field of Scientific and Technical Research

- Title: An integrated European Platform for Pancreas Cancer Research: From Basic Science to Clinical and Public Health Interventions for a rare disease

- Duration: 2013-2016

○ FP7-Helath-2012-Innovation

- Title: RARE Best practices - Best Practice and knowledge sharing in the clinical management of rare-diseases

- Duration: 2013-2016

○ FP7-ICT-FET-F

- Title: Information and Communication Technology Future of Medicine /ITFoM

- Duration: 2011-2012

- EU DG SANCO

- European Agency for Health Consumers 
- Public Health Genomics European Network II (PHGEN II),

- Title: European Best Practice Guidelines on Quality Assurance Provision and Use of Genome-based Information and Technologies.

- Duration: June 2009 to November 2012

- Canadian

- APOGEE Net CanGeneTest - A Research and Knowledge Network on Genetic Health Services and Policy.

- Pilot Project Title: Efficient integration of genome-based technologies into the healthcare system.

- Duration: March 2011 to March 2015

\section{Other related articles/conference proceedings}

- Brand A, Lal J, Malats N, Gutierrez-Ibarluzea I. The Public Health Genomics European Network. The Newsletter of the British Society for Human Genetics 2013, 48:53-55.

- Brand A, Lal J. Declaration of Rome-European guidance on Public Health Genomics. 5th European Public Health Conference. European Journal of Public Health 2012, 22(2):16.

\section{Interviews related to the model}

- TV Interview sporting a discussion along with colleagues regarding Public Health Genomics (1505-2012, 11:30-14:00) for the ARTE TV network.

- Thirty minutes telephonic interview on the harmonization strategies for HTA reimbursement and regulatory approval by Prof. Ron Goeree (McMaster University, Canada) and Michael Drummond (University of York). (06-12-2012, 15:00-15:30)

The Learning-Adapting-Leveling model has also been incorporated in other currently submitted FP7s as well as other grants including the current DG SANCO 'Call for tender $n^{\circ}$ EAHC/2013/Health/09 concerning pilots on early dialogue between health technology assessors and healthcare product developers during the development phase of medicinal products and medical devices' which directly relate to the interactions of the model. Furthermore, a Universiteitsfond Limburg SWOL grant to organize a workshop around the Learning-Adapting-Leveling model has been accepted. This workshop will involve high level stakeholders to familiarize themselves with the model and to implement it. In addition, other papers in submission and preparation emphasis the model. In particular from the reports 


\section{| GENERAL DISCUSSION}

listed, the report from the Irish Presidency of the EU incorporates the model. Also the incorporation of the model in the European Science Foundation's Forward Look report and the developed European best practice guidelines of PHGEN II at EU level emphasize the extensive proliferation of the model in the EU.

\section{Ongoing Adaptions}

On the other hand, there are issues which still have to be addressed. Preferentially, government driven regulatory requirements concerning the Learning-Adapting-Leveling model would be stimulating for the adoption of the model, however may turn out as stated in the EU best practice guidelines above. Also, having the push of the technology from the market and the pull is from the society or healthcare based around the need is ideal. Therefore, it is up to the academic-industrial complex to voluntarily implement the model in their setting. It can be said here, that the broadly defined public-private partnerships are an important component to spread this voluntary implementation to a standard eventually as these partnerships convey the momentum from on section to another. As a consequence, this can bolster possible future government driven regulatory requirements. However, as mentioned in chapter II, the EU DG SANCO has placed a call for these early interactions understanding the importance of it. The Learning-Adapting-Leveling model is an applicant in that call.

The model seems simply straightforward. Nevertheless, the public health assessment tools (HNA, HTA and $\mathrm{HIA}$ ) have to be compatible to each other as well as technology transfer as mentioned in chapters I and II. These tools have their own timeframes and subjective methodologies. This can lead of redefining the roles of these tools. This will only be achieved through the interactions within the Learning-AdaptingLeveling model possibly leading to organizational and policy-wise changes to synergize the approach. Coming back to HTA, it should be noted like HNA and HIA, although quite widely used in several countries, there are regions where agencies with alternate names and functions carry out the assessments. It goes without saying that in that case within the Learning-Adapting-Leveling model those agencies will replace the public health assessment tools mentioned for the assessment. Nonetheless, the technology transfer professionals will most likely have to always approach the HNA, HTA and HIA professionals to activate the model. Given its wide lobbying, the model could be eventually considered a standard in some regions whereas in other regions it may take time or be rejected.

However, the model can still be applied internally by technology transfer professionals, if the current landscape does not allow the development of the innovation network. Through this the academic- 
industrial complex can ensure that even though an innovation network or public-private partnership does not exist, they nonetheless are able to conform to perceived health policy needs. Internal assessments should be not considered a replacement to the original design as it is important that health policy is on board for real-time acceptance. Furthermore, development of public-private partnerships is beneficial in the long run. Importantly, the core problem of delayed uptake or lack of effective and efficient real-time uptake of genome based (information and) technologies is in fact the lack of communication or non-synergy between the academic-industrial complex and health policy. Therefore, internal assessments should be not a replacement.

The term technology transfer can be described in simplistic terms as to transfer the technology from one setting to another setting. However, the term itself is mostly used in the valorization and commercialization of technologies, therefore does not constitute healthcare implementation. Although there may be issues between the players of technology transfer, given its extensive usage and popularity, the problems are minor and can be neglected. Also within technology transfer the drug discovery phase timeline issues as mentioned in chapter I are not due to the organizational issues of technology transfer rather the proof of concept of the drug being developed. It should also be noted as stated in chapter III that technology transfer professionals should present the best or few of the best options to health policy rather than showing all on the table for assessment as this can result in rejection.

Regional valorization systems as indicated in section 1 seem to have some of the ingredients from industry to policy, which can bring in the question, why it was not considered an overarching framework. The system itself apart from being not popular and not proven importantly has no defined method or defined actors. Furthermore this system works on the policy aspect for development for only business conditioning or favorable conditions. Also it does not cover the whole pipeline (from bench to healthcare) and is only focused on regional structures. In addition, it does not have any systematic proven approaches like HTA. Conclusively, it is far away from being useful as the healthcare perspective is completey lacking. The Learning-Adapting-Leveling model does in fact take these aspects into consideration and goes beyond business conditioning for conditioning of the healthcare benefits. Also the regional valorization system is in fact covered within the Learning-Adapting-Leveling model with regard to its interactions. 


\section{| GENERAL DISCUSSION}

The mentioned reference frames of the Learning-Adapting-Leveling model should be considered more indicative than absolute as stated in chapter III. With respect to the applicability of the technology and relevance to the end user and the actor implementing the model, certain criteria of the business aspect of the value of information can be overlooked. Also the process of answering the reference frames in the positive will make the chances of success more likely; however the process itself is not defined in the thesis. Again this is dependent on the actor using the model. The reference frames can be considered as a prelude to the actual development of the innovation network.

Timelines are currently hypothetical in chapter III as the model has only started its proof of concept yet which is currently ongoing. Although the ideal of 5-10 years seem good, it may not be the case when the model is executed. In fact, the initial timeframe could be much longer. However, in the long run over iterations the timeframe can be reduced and optimized. Even for the technology transfer pipeline, the timeframe could increase due to the additional consultations and feedback loops with the HNA, HTA and HIA professionals. Also structural conformity issues between the different agencies and professionals involved may take its initial toll. Nonetheless, real-time uptake by healthcare systems should be more likely once the technology is rolled out and is more advantageous when compared to the time lost from market to healthcare. Another issue is that relevant lobbying in the Learning-Adapting-Leveling model brings in ethical issues of varied interests of stakeholders. As indicated in chapter I, the decision makers should apart from the lobbied case of the academic-industrial complex and their own perceived point of view, use opinions of third parties like patient groups and ethical boards to ensure only relevant technologies reach the end user.

Coming back to the structural conformity mentioned in the previous paragraph, apart from the issues of conformity amongst the different agencies, HTA for example is mainly focused on a population level as mentioned in chapter VI, given the new development of the European best practice guidelines as in chapter VII, the shift seems to move from clinical utility to personal utility. As a consequence, HTA will have to shift from assessment at a population level to an individual level. Although currently this seems a long term organizational tour, it is required to shift to personalized medicine as given in the guidelines. Nonetheless, HTA analysis can still happen for personalized interventions however will be at a more stratified level than individual which is feasible for the near future and for the model. Nonetheless, conformity issues between agencies remains. 
Also as mentioned in chapter I, the model does not address the phasing out of the technology although it is pointed out that the same model can be used to phase out as it is used to phase in. This is dependent on who is the user, for example, the healthcare professional or industry. The prior would be more concerned with the phasing out. Nevertheless, it would be advantageous for the academicindustrial complex to also pay attention to the phasing out of the technology as it would present a better case for simultaneously phasing in a technology. Although this has not been expanded upon in the chapter, it can be briefly emphasized. The interactions between technology transfer and the public health assessment tools through the development of the innovation network will eventually lead to public-private partnerships. In parallel to introducing a technology through the assessments, the technology transfer professionals should build a case based on those assessments or based on the early assessment of that integrated technology, why it should be made obsolete while introducing their technology. A SWOT (Strengths, Weaknesses, Opportunities and Threats) analysis and comparative analysis can build a case in a broad and quick way for the phasing out of a technology. This can be done simultaneously while developing their technology through the framework of the Learning-AdaptingLeveling model. Another aspect of this model is that it can be used in an adapted form to propel relevant non-genome-based technologies into healthcare or other organizations.

Although the issue of bottleneck of delay is the problem on the major part, the model in some instances is not required in a smaller setting. For example, some university medical centers develop technologies within their own settings and implement it into their own settings directly without going through the general approval protocols. Some of such developed technologies go as far as not applying for a CE marking as the technology is not being officially categorized as diagnostics. These technologies are diffused readily into that healthcare settings and related networks. These developed technologies are generally built in collaboration with industry. On the other hand sometimes, industry developing prototypes and using their contacts let these university medical centers test the feasibility of the technology and adapt the technology in-house. However with current ongoing scenarios, the CE marking is being discussed with industry regarding its possibility of potential mandatory application, which may change the approach of such university medical centers making it more feasible for the LearningAdapting-Leveling model. However, if willing the medical centers, the model can be differentially applied in these settings to broaden the scope and reach of these developing technologies also making it easier for future official assessments. In the current status, the assessments can be more internal than official and later on approached to the official channels as per requirement, for example if they want to move 


\section{| GENERAL DISCUSSION}

from related networks usage to wide implementation. Another perspective to this issue is that quality assurance is becoming very important. In the near future, also technologies applied in hospitals (medical centers) need to have an HTA and systematic assessments. Thus, no in-house testing will be possible eventually. This is a consequence of EU talks after recent scandals (like silicon implants) and is part of the new EU directive on medical devices and IVD new directive. This contributes to the importance of the model.

The concept of the game theory has been widely used in several fields of applications including biology. The interactions between technology transfer of the academic-industrial complex and public health assessment tools of the decision makers does involve complex interactions. Therefore a game theory approach to study this will be an interesting endeavor. With regard to the interactions between technology transfer and the public health assessment tools as two separate units, the concept of noncooperative game theory would be interesting to investigate. Furthermore, the interactions of the academics with industry (academic-industrial complex) and the internal interactions between HNA, HTA and HIA within the public health assessment tools will be interested to analyze with cooperative game theory.

The Learning-Adapting-Leveling model has not been completely tested although is being tested on different projects for the proof of concept. Until the proof of concept is accomplished, the scenarios and probabilities mentioned above although seem likely are still hypothetical. Therefore, it is very important for the sustainability of the model to have results on its first run which is currently an ongoing process for the next few years. 


\section{Chapter-wise Addressed Thesis Objectives}

The general objectives of the thesis can be summarized as have been answered in the mentioned chapters (when compared to the specific objectives and conclusions of Part A and Part B):

- parta

- Chapter I

- Identify the reason behind the backlog or bottleneck of effective and efficient real time integration of health innovations/interventions (genome-based technologies)

- Address the issue of this bottleneck by proposing a framework covering the whole spectrum of translational research possibly through integration and development of (new) concepts

- Defining the concept of valorization in public health genomics

- Chapter II

- Demonstrating reasons why certain tools are not relevant to the framework

- Addressing the confusion behind different concepts in translational research and showing the relations between them including conceptual development of the placement of tools within the translational research pipeline

- Chapter III

- Developing the protocol of the developed framework

- pART B

- Chapters V and VI

- Giving examples where the new framework can be implemented

- Chapter VII

- Contribution to the development of the European best practice guidelines on genome-based information and technologies 



\section{Section 4}

\section{Concluding Remarks and Future Prospects}

Adapted and Expanded From:

LaI JA.

Addressing the backlog of genome-based technologies in healthcare. The Newsletter of the British Society for Human Genetics 2013, (48):59-60. 
Personalized healthcare sets off a new era of healthcare that currently focuses on the individual genomic signature of patients interacting dynamically with other health determinants such as environmental and lifestyle factors to create a more personalized approach at a much earlier stage to healthcare. The core diagnostic/therapeutic segment of the market comprised primarily of drugs, medical devices and diagnostics is expected to reach $f 26$ billion by 2015. Improvements in genome-based technologies are primarily responsible for this staggering growth. The market is pioneered by small to medium enterprises (SMEs) regarded as the true innovator of personalized diagnostics market, highly contributing to benefits for society as stated in Europe's growth strategy EU 2020. However, both historically and currently we see that the timely uptake and implementation of these relevant SME-based genomics-related applications in real time is negligible. The average time to diffusion in healthcare systems is over 10-20 years even with marketing approval (FDA, CE, CE-IVD, etc.). As a result and given the exponential fast pace of technological development, by the time a relevant application is integrated into the healthcare system, it is considered less relevant as a more effective and efficient application becomes available on the market. Subsequently, both businesses and the population (e.g., patients in need of new and more accurate clinical diagnostics) are at a disadvantage. This can increase the burden of disease as well as become a development hurdle for companies in the diagnostics market causing significant problems, i.e. product failure connecting to loss of employment, capital and IP.

Based around this bottleneck of healthcare integration the unique Learning-Adapting-Leveling model was developed at the Institute for Public Health Genomics (IPHG) at Maastricht University. The innovative nature of this model is that for the first time it brings together in parallel two never before connected yet seemingly striking activities or worlds. The first being technology transfer, which is used by industry to translate academic knowledge, patents and applied research into marketable products and the second being the public health assessment tools. The latter is used by decision makers to assess technologies on the market for healthcare implementation/acceptance, guidelines and reimbursement. The Learning-Adapting-Leveling model identifies non-synergy between these two different entities as the reason for the bottleneck of technological integration. The model assesses the feasibility of the developing technology from the start to near the end of the technology maturation process for real-time uptake by healthcare systems and policy guidelines, thereby give real-time recommendations to 
compensate for any gaps in the process prior to launching the product and to build contact with policy. This model encourages early-on involvement of all stakeholders including doctors, industry, patient groups, investors, insurance companies, HTA professionals, etc. leading towards a possible cross-talk and public-private partnership. The model also takes into account and assesses the Value of Information and does control analysis of possible gaps defined through the 10 essential public health tasks defined by the Institute of Medicine (IOM) in 1988 and adapted to public health genomics within the Public Health Genomics European Network (PHGEN). As a consequence it can be considered an overarching framework given its extensive coverage of various widely regarded tools.

The model has been officially integrated in the European best practice guidelines for QA, provision and use of genome-based information and technologies, FP7s and a few SMEs as well as the EU flagship pilot project ICT Future of Medicine (www.itfom.eu). The model is also being used with SMEs and other projects as well as grants and publications. Also a Joint Action is being planned for PHGEN II for the implementation of the European best practice guidelines or in other words the implementation of the model. Furthermore, the model has been published in high level reports like the Cyprus and Irish Presidency and the European Science Foundation's Forward Look report among others. The model has been presented in the form of talks and poster presentations at international conferences and lectures. Approaches even by contacts outside the EU (US and Australia) with people interested in the model have been seen. Moreover, a grant from Limburg Fond has been obtained to organize a workshop on the LAL model with participants from the academic-industrial complex, policy makers, hospitals and health services on the regional level. The model has been lobbied for in the EU member states. Recently, the EC has come up with a very specific tender on HTA interactions with technology developers as mentioned in the general discussion section, which completely fits to the model showing the direction the EU is interested in. Therefore, the sustainability of the model seems feasible and possibly could become the standard for implementation of technologies to public health and healthcare. The next step of the model is to test its proof of concept with pilot studies.

Although the model still may have adaption issues as mentioned in the general discussion section, it is nonetheless the first translational model created within Public Health Genomics and the only one being used at the Institute for Public Health Genomics for valorization concepts. Also, this model fulfills accurately the definition of Public Health Genomics. The model creates translational research from basic sciences to public health and from technology development to technology transfer. Most importantly, 


\section{CONCLUDING REMARKS AND FUTURE PROSPECTS}

the model is a new specialization (valorization) within the field of Public Health Genomics. As the field of Public Health Genomics is more into 'the responsible and effective translation...' the sub-specialization of valorization in public health genomics is more into the 'process of realization of added value bioproducts (genome-based technologies) in the domain of public health...'. Realization is from the perspective of identifying and understanding the importance and relevance of the bioproduct, i.e. any kind of technology, for public health and healthcare purposes, thereby implementing them.

The Learning-Adapting-Leveling model based on the previous chapters has been proposed to be the overarching framework in translational research for integration and implementation from bench to bedside and beyond, i.e. into healthcare, through early-on interactions of all stakeholders. Through the process the stakeholders 'learn' from each other, 'adapt' or conform to each other's requirements and through the innovation network and developed public-private partnerships 'level' the modes of communication to a single platform. This is the essence of the Learning-Adapting-Leveling model. The model conceptually answers the original objective of the thesis regarding the bottleneck of the effective and efficient real-time integration of genome-based information and technologies into healthcare.

Conclusively the thesis has developed a conceptual framework for the whole pipeline of translational research i.e. from technology transfer to healthcare integration, using the concept of valorization in public health genomics and demonstrated it with examples of potential applications. However the proof of the model is an ongoing endeavor with various partners over the next few years. 


\section{Section 5}

\section{Miscellaneous}

Summary

Dutch Summary

Hindi Summary

Curriculum Vitae

Publications

Acknowledgements 



\section{Summary}

Public Health Genomics is the responsible, effective, efficient and timely translation of genome-based information and technologies into health policies and practice for the benefit of population health. Being complementary to the interventions in health protection and health promotion, public health genomics also demonstrates that the current public health interventions move towards personalized healthcare including personalized medicine. In the past decade we have seen an exponential growth of personalized medicine applications onto the market with the US market alone estimated to be at \$454 billion by 2015 . The advent of personalized medicine and healthcare can find its roots in computational power through 'omics' research as well as systems biology and systems biomedicine. Importantly this push has been from active participation of individuals through social media and the internet. However, it is noticed that the implementation into healthcare systems of such personalized applications in the form of genomebased information and technologies seems to have a huge backlog. Consequently both industry and the citizens are at disadvantage. The thesis aims to address the issue of the backlog and bottleneck of the effective and efficient integration of genome-based information and technologies (including personalized medicine applications) from the bench (lab) through the market to the bedside and beyond (healthcare) covering the whole spectrum of translational research of (personalized) healthcare. The hypothesis is that given the exponential increase in genome-based technological innovation, systems in place for technological integration into the healthcare practice still have a backlog in real time. The thesis therefore projects to gain knowledge about current methodological apparatuses in place, identification of gaps/problems and issues in healthcare systems, see possible integration of relevant tools and give recommendations as well as potential application of the recommendations. Furthermore, certain existing concepts and ideas have overlaps, and the thesis also aims to develop a consistency of the relation of different tools to avoid confusion thereby bringing in new concepts in valorization. In addition, the current existing European guidelines are not compatible for implementation of genomebased technologies; therefore the thesis also contributes to the recently developed and endorsed European best practice guidelines (PHGEN II), which will be implemented in the next few years in all EU member states. 
With respect to the aims mentioned above, section 1 describes and builds the case for the above mentioned problem statement and reviews the state of the art. In section 2, PART A of the thesis deals with the development of theoretical concepts.

Within PART A, chapter I deals with setting the stage of the issue as the lack of communication or nonsynergy between the academic-industrial complex and decision makers. It was identified that both use different tools for commercialization and implementation respectively, namely technology transfer (TT) and the public health assessment tools (PHAT) in the form of health needs assessment (HNA), health technology assessment (HTA), and health impact assessment (HIA). Furthermore, the chapter defined the concept of valorization in Public Health Genomics as the process of realization of added value (bio)products in the domain of public health for benefit of the population and healthcare systems. The chapter addressed the bottleneck by developing a conceptual framework known as the LearningAdapting-Leveling model. This model brings for the first time technology transfer and the public health assessment tools for cross talk to conform to each other's needs.

Chapter II elaborates on all the widely used concepts of translation and implementation of genomebased technologies such as personalized health applications. These include valorization, regional valorization system, open innovation, technology transfer, drug discovery, spin-offs from the commercial perspective from bench to market. From market to healthcare concepts include HNA, HTA, HIA, ACCE, EGAPP, T1-T4, PHG Enterprise and the PHG wheel. The chapter clarifies the concepts of different tools and demonstrates the place of different tools within other concepts and clears the confusion. It elaborates that for the commercialization technology transfer covers all aspects of translation to the market including valorization, regional valorization system, open innovation, drug discovery and spinoffs. It also demonstrates why certain tools were left out of the developed framework (ACCE, EGAPP, T1T4, PHG Enterprise) due to limited scope and overlaps with existing widely regarded tools.

Chapter III develops the complete detailed protocol on how to use the Learning-Adapting-Leveling model. This was stated as after initial research and development the technology transfer professionals contact the HNA, HTA and HIA professionals in the same order involving feedback loops and going back to the drawing board before going to the next professional (HNA to HTA to HIA). Throughout the process the reference frames of value of information and the public health genomics wheel is taken into account. This could lead to broadly defined public-private partnerships and can be optimized over time. Also this 
chapter addresses issues of timeframe as well as compatibility issues. Chapter IV gives the general conclusion of Part A based on the specific objectives defined.

PART B deals with the potential implementation scenarios of the developed framework.

Within Part B, chapter $\mathbf{V}$ describes the complexity of biology as a result of '-omics' and the introduction of systems biology and its relation to personalized medicine and healthcare. It proposes the implementation of the developed framework using an example of a current EU flagship pilot project ICT Future of Medicine (ITFoM).

Chapter VI gives an overview of the current state of the art of host genetic markers in relation to Chlamydia trachomatis infection. The potential translational and clinical value of adding diagnostic host genetic marker profiles on the basis of infection and inflammation to the current clinical management of subfertility was investigated. The chapter proposes a new clinical diagnostic approach supplementing traditional Chlamydia trachomatis serological tests, and investigates how the Learning-Adapting-Leveling model can be of value and provide insight to see whether these host genetic markers can be translated into public health

Chapter VII contributes to the development of the European best practice guidelines for quality assurance, provision and use of genome-based information and technologies (PHGEN II) and clears certain concepts. Here the thesis demonstrates the incorporation of the developed framework in the European policy guidelines. Chapter VIII gives the general conclusions of Part B based on the specific objectives defined.

Section 3 discusses in detail the conceptual development of the framework and discusses the issues of the previous chapters and conceptualizes beyond the status quo and concludes the model's overarching reach and the development of the conceptual framework from technology transfer to healthcare integration. Section 4 derives the final conclusion over the complete thesis and states that the framework developed answers the objectives of the thesis regarding the bottleneck of real-time effective and efficient integration of genome-based information and technologies, although the proof of concept is a currently ongoing project over the next few years with collaborators. Section $\mathbf{5}$ gives the summary of the thesis, the $\mathrm{CV}$ of the thesis author as well as list of publications and acknowledgements. 



\section{Samenvatting (Dutch Summary)}

Public Health Genomics is de verantwoordelijke, effectieve, efficiënte en tijdige vertaling van genoomgebaseerde informatie en technologie naar gezondheidsinstellingen en gezondheidszorg ter bevordering van de volksgezondheid. Terwijl Public Health Genomics bijdraagt aan de interventie in gezondheidsbescherming en bevordering van de gezondheid, laat het ook zien dat de hedendaagse interventies in Public Health veranderen in gepersonaliseerde gezondheidszorg, en daarmee gepersonaliseerde geneeskunde. In de afgelopen tien jaar was er op de markt een exponentiële groei waarneembaar in gepersonaliseerde geneeskundige toepassingen, met de markt van de Verenigde Staten alleen al geschat op een waarde van \$454 miljard tegen het jaar 2015. De komst van gepersonaliseerde geneeskunde en gezondheidszorg vindt zijn oorsprong in zowel rekenkracht door onderzoek naar 'omics', alsmede systeem biologie en systeem biomedische wetenschappen. Het is belangrijk te weten dat de aanzet daarvan afkomstig is uit de actieve betrokkenheid van individuen door sociale media en internet. Er is echter bemerkt dat de implementatie in gezondheidszorgsystemen van dergelijke gepersonaliseerde toepassingen, in de vorm van genoomgebaseerde informatie en technologie, een enorme achterstand heeft. Als gevolg worden zowel de industrie als de bevolking benadeeld. Deze thesis heeft als doel het ter sprake brengen van het probleem van de achterstand en het knelpunt van de effectieve en efficiënte integratie van genoomgebaseerde informatie en technologie (inclusief gepersonaliseerde geneeskundige toepassingen) vanaf de onderzoekstafel (laboratorium) via de markt naar het ben van de patiënt en verder (gezondheidszorg), om het gehele spectrum aan translationeel onderzoek naar (gepersonaliseerde) gezondheidszorg te behandelen. De hypothese is dat gezien de exponentiële toename in genoomgebaseerde technologische innovatie, de bestaande systemen voor technologische integratie in de praktijk van gezondheidszorg een voortdurende achterstand hebben. Deze thesis stelt zich ten doel om kennis te verwerven van hedendaagse methodologische systematiek, missende informatie of problemen binnen gezondheidszorgsystemen te identificeren, mogelijke integratie van relevante hulpmiddelen in kaart te brengen en zowel aanbevelingen als potentiële toepassing van de aanbevelingen te doen. Verder hebben bepaalde bestaande concepten en ideeën overlap, en de thesis heeft tevens als doel om éénduidige hulpmiddelen te ontwikkelen, en zal daarbij nieuwe concepten van valorisatie introduceren. Bovendien zijn de huidige bestaande Europese richtlijnen niet compatibel voor implementatie van genoomgebaseerde technologie; daarom draagt de thesis ook bij aan de recent ontwikkelde en doorgevoerde European best 
practice guidelines (PHGEN II), welke gedurende de komende jaren geïmplementeerd zullen worden in alle EU lidstaten.

Met betrekking tot bovengenoemde doelstellingen, beschrijft en onderbouwt sectie 1 van de thesis de eerst genoemde probleemstelling, en beoordeelt de huidige stand van zaken. In sectie $\mathbf{2}$ behandelt DEEL A de ontwikkeling van theoretische concepten.

Binnen DEEL A behandelt hoofdstuk 1 het introduceren van het probleem van gebrek aan communicatie en non-synergie tussen het academisch-industriële complex en de besluitvormers. Analyse liet zien dat beiden verschillende hulpmiddelen gebruikten voor commercialisatie en implementatie, namelijk technology transfer (TT) en de public health assessment tools (PHAT) in de vorm van health needs assessment (HNA), health technology assessment (HTA), en health impact assessment (HIA). Verder stelde het hoofdstuk het concept van valorisatie in Public Health Genomics vast als het realisatie-proces van (bio)producten met toegevoegde waarde in het domein van volksgezondheid ten behoeve van de bevolking en gezondheidszorgsystemen. Het hoofdstuk benoemt het knelpunt door het ontwikkelen van een conceptueel kader dat we kennen als het Learning-Adapting-Leveling model. Dit model brengt voor het eerst technologische overdracht en de beoordelingshulpmiddelen van de volksgezondheid samen om elkaar aan te vullen.

Hoofdstuk II gaat dieper in op alle veel gebruikte concepten van translatie en implementatie van genoomgebaseerde technologieën zoals gepersonaliseerde toepassingen in de gezondheidszorg. Hiertoe behoren valorisatie, het regionale valorisatiesysteem, open innovatie, technologische overdracht, farmaceutisch onderzoek, en spin-offs vanuit commerciële perspectief van de onderzoekstafel tot de markt. Concepten van de markt naar gezondheidszorg omvatten HNA, HTA, HIA, ACCE, EGAPP, T1-T4, PHG Enterprise en het PHG wiel. Het hoofdstuk verduidelijkt de concepten van verschillende hulpmiddelen en demonstreert de plaats die verschillende hulpmiddelen innemen binnen andere concepten. Het gaat verder in op het feit dat voor commercialisatie, de technologische overdracht alle aspecten van vertaling naar de markt beslaat, inclusief valorisatie, het regionale valorisatiesysteem, open innovatie, farmaceutisch onderzoek en spin-offs. Het toont ook aan waarom bepaalde hulpmiddelen werden weggelaten uit het ontwikkelde kader (ACCE, EGAPP, T1 - T4 , PHG Enterprise) vanwege de beperkte reikwijdte en overlappingen met bestaande, alom bekende hulpmiddelen. 
Hoofdstuk III ontwikkelt het complete gedetailleerde protocol rond de vraag hoe het Learning-AdaptingLeveling model gebruikt moet worden. Dit werd opgesteld aangezien de professionals in de technologische overdracht, na het beginnende onderzoek en ontwikkeling, contact opnamen met de HNA, HTA en HIA professionals in dezelfde volgorde, waarin zij feedback rondes betrokken en terugkeerden naar de eerste fase voordat zij verder gingen naar de volgende professional (HNA naar HTA naar HIA). Gedurende dit proces werden de referentiekaders informatie-waarde en het Public Health Genomics Wheel in acht genomen. Dit zou kunnen leiden tot breed gedefinieerde publiekprivate partnerschappen, hetgeen later steeds verder geoptimaliseerd kan worden. Dit hoofdstuk behandelt ook de problemen van het tijdskader, alsmede compatibiliteitsproblemen. Hoofdstuk IV geeft de uiteindelijke conclusie van DEEL A, gebaseerd op de specifiek gedefinieerde doelstellingen.

DEEL B behandelt de potentiële implementatie scenario's van het ontwikkelde kader.

Binnen DEEL B omschrijft Hoofdstuk $\mathbf{V}$ de complexiteit van biologie als resultaat van '-omics', en de introductie van systeem biologie en haar relatie tot gepersonaliseerde geneeskunde en gezondheidszorg. Het stelt de implementatie van het ontwikkelde kader voor door het voorbeeld van het belangrijke huidige EU proefproject ICT Future of Medicine (ITFoM) aan te halen.

Hoofdstuk VI geeft een overzicht van de huidige stand van zaken wat betreft de genetische merker in relatie tot de Chlamydia trachomatis infectie. De potentiële translationele en klinische waarde van het toevoegen van diagnostische genetische merker profielen, op basis van infectie en ontsteking, aan het huidige klinische management van verminderde vruchtbaarheid werd onderzocht. Het hoofdstuk stelt een nieuwe klinisch diagnostische benadering voor ter aanvulling op de traditionele serologische test op Chlamydia trachomatis, en onderzoekt hoe het Learning - Adapting - Leveling model van waarde kan zijn en inzicht kan geven om te zien of deze genetische merkers naar de volksgezondheid kunnen worden vertaald.

Hoofdstuk VII draagt bij aan de ontwikkeling van de Europese richtlijnen voor kwaliteitsborging, levering en het gebruik van het genoomgebaseerde informatie en technologieën (PHGEN II) en verduidelijkt specifieke concepten. Hier laat de thesis de incorporatie van het ontwikkelde kader in de Europese richtlijnen zien. Hoofdstuk VIII geeft de algemene conclusies van DEEL B, gebaseerd op de specifiek gedefinieerde doelstellingen. 
Sectie 3 gaat uitgebreid in op de conceptuele ontwikkeling van het kader en benoemt de problemen uit de voorgaande hoofdstukken, conceptualiseert verder dan de status quo, en concludeert het overkoepelende bereik van het model en de ontwikkeling van het conceptueel kader van de overdracht van technologie tot integratie in gezondheidszorg. Sectie 4 trekt de uiteindelijke conclusie vanuit de gehele thesis en stelt dat het ontwikkelde kader de doelstellingen van de thesis, betreffende het knelpunt van werkelijk effectieve en efficiënte integratie van genoomgebaseerde informatie en technologieën, beantwoord, hoewel het bewijs van het concept momenteel work-in-progress is. Sectie 5 geeft een samenvatting van de thesis, de CV van de auteur van deze thesis alsmede een lijst van publicaties en dankwoord. 


\section{सारांश (Hindi Summary)}

जन स्वास्थ्य जीनोमिक्स, जनता के स्वास्थ्य लाभ के लिए स्वास्थ्य नीतियों और व्यवहार में जीनोम आधारित सूचना और प्रौद्योगिकी के जिम्मेदार, प्रभावी, कुशल और सामयिक अनुवाद है। स्वास्थ्य सुरक्षा और स्वास्थ्य को बढ़ावा देने में हस्तक्षेप करने के लिए पूरक होने के नाते, जन स्वास्थ्य जीनोमिक्स भी मौजूदा जन स्वास्थ्य उपायों, व्यक्तिगत दवा सहित व्यक्तिगत स्वास्थ्य की दिशा मे एक प्रभावी कदम दर्शाता है। पिछले एक दशक में हमने अमेरिका के बाजार मे अकेले 2015 तक \$ 454000000000 में होने के अनुमान के साथ बाजार पर व्यक्तिगत चिकित्सा अनुप्रयोगों की एक व्याख्यात्मक वृद्धि देखी है। व्यक्तिगत चिकित्सा पद्धति और स्वास्थ्य सेवा के आगमन से ओमिक्स अनुसंधान के साथ ही जीव विज्ञान प्रणाली और प्रणालियां, बायोमेडिसिन के माध्यम से लेखाव्यक्ति मे अपनी जड़ो को पा सकता है। यह महत्वपूर्ण कार्य सामाजिक मीडिया और इंटरनेट के माध्यम से व्यक्तियों की सक्रिय भागीदारी से किया गया है। हालांकि यह जीनोम आधारित सूचना और प्रौद्योगिकी के रुप मे इस तरह के व्यक्तिगत उपयोग,स्वास्थ्य प्रणाली में एक संचित कार्य है। जिसके परिणाम स्वरुप दोनो उद्योग और नागरिको को हानि हुई है। इस थीसिस का यह लक्ष्य है कि संचित कार्य तथा अन्य रुकावटों कि समस्या का समाधान हो सके, ताकि उपयोगी तथा प्रभावशाली जीनोम आधारित तकनीकी सूचना और अभियोग का एकीकरण हो सकें, जिससे कि प्रयोगशाला से स्वास्थ्य सेवा बाजार हेतु सभी तरंगो में स्वास्थ्य सेवा अनुसंधान का अनुवाद हो सके। कल्पना की जाती है कि जीनोम आधारित तकनीकी मे घातांकी बढ़ोत्तरी तथा तकनीकी एकीकरण, स्वास्थ्य सेवा कार्य प्रणाली मे संचित कार्य है। इसलिए यह शोध-पत्र आज के समय में प्रणाली संबंधित उपकरणों, अन्तराल/समस्या की पहचान तथा स्वास्थ्य सेवा क्रम मामले, उपर्युक्त उपकरणों का एकीकरण, तथा संम्भवता उपयोग के प्रोत्साहन को संबोधित करता है ताकि ज्ञान की बढ़ोत्तरी हो सके। इसके अतिरिक्त कुछ ऐसी मौजूदा विचार धाराएँ है, जो एक दूसरें पर अतिव्याप्त है। यह शोध पत्र मूल्य वृद्धि में नई अवधारणाओं में लाने के भ्रम की स्थिति से बचने के लिए विभिन्न उपकरणों के संबंध मे एक अनुकूलता विकसित करने का एक प्रयास है। इसके अलावा मोजूदा यूरोपीय दिशानिर्देश जीनोम आधारित प्रौद्योगिकी के कार्यान्वयन के लिए संगत नही है। इसलिए इस थीसिस के परिणामों को अगले कुछ वर्षो मे लागू किया जाएगा, जो हाल मे ही विकसित और समर्थन प्राप्त यूरोपीय सबसे अच्छा अभ्यास दिशा निर्देशों (पीएचजीइएन द्वितीय) के लिए योगदान के रुप मे यूरोपिय संघ के सदस्य देशों/राज्यों में अगले कुछ वर्षो में लागू की जा सकती है।

ऊपर दिये गए उल्लेख जो खण्ड-1 में वर्णित है उपरोक्त समस्याओं के बयान के लिए मामले को मजबूत बनाता है और कला की स्थितिज की समीक्षा करता है। शोध-पत्र के खण्ड-2, भाग अ में सैद्धान्तिक धारणा के अविष्कार का वर्णन है। 
भाग-अ के भीतर प्रथम अध्याय में शैक्षणिक औद्योगिक परिसर और निर्णय निर्माताओं के बीच संचार या गैर तालमेल की कमी के रूप में इस मुद्दे को मंच पर स्थापित करने का समायोजन है। व्यवासायिक और कार्यान्वय के लिए दोनों का उपयोग विभिन्न उपकरणों अर्थात प्रौद्योगिकी हस्तान्तरण (टी०टी०) और स्वास्थ के रूप में सार्वजनिक स्वास्थ मूल्यांकन उपकरण (फैट) स्वास्थ सम्बन्धी (एच०एन०ए०) तकनीकी प्रौद्योगिकी आंकलन (एच०टी०ए०) की पहचान करना आवश्यक है। इसके अलावा इस अध्याय में जोड़ मूल्य की प्राप्ति (जीव) जनसंख्या और स्वास्थ्य प्रणालियों के लाभ के लिए सार्वजनिक स्वास्थ्य के क्षेत्र में उत्पादो को प्रक्रिया के रुप में जन स्वास्थ्य जीनोमिक्स में मूल्य वृद्धि की अवधारणा को भी परिभाषित किया गया है। यह अध्याय लर्निंग एडापटिंग लेवलिंग मॉडल के रुप में एक वैचारिक ढांचे के विकास को भी संबोधित करता है। यह मॉडल, एक दूसरे की जरुरतो के अनुरुप पहली बार प्रोद्योगिकी हस्तांतरण और सार्वजनिक स्वास्थ्य मूल्यांकन उपकरण की बात करता है।

द्वितीय अध्याय में व्यक्तिगत स्वास्थ्य अनुप्रयोगों के रुप में जीनोम आधारित प्रौद्योगिकी का अनुवाद और कार्यान्वयन के सभी पहलुओं का व्यापक रुप से इस्तेमाल किया गया है। यह अवधारणा बताती है कि प्रौद्योगिकी बेंच से बाजार को व्यावसायिक नजरिए से मूल्यवृद्धि,क्षेत्रियमूल्यवृद्धि प्रणाली, खुला नवाचार, प्रौद्योगिकी हस्तांतरण दवाओं की खोज उपोत्पाद शामिल है। बाजार से स्वास्थ्य अवधारणाओं (एच०एन०ए०) (एच०टी०ए०) (एच०आइ०ए०) (ए०सी०सी०ई०) (इ0जी०ए०पी०पी०) (टी1)-(टी4) (पी०एच०जी०) उद्यम और (पी०एच०जी०) चक्र शामिल है। इस अध्याय मे विभिन्न उपकरणों की अवधारणाओं को स्पष्ट किया है यह अन्य अवधारणो के भीतर विभिन्न उपकरणों के स्थान को दर्शाता है और भ्रम की स्थिति को साफ करता है। यह व्यावसायीकरण प्रौद्योगिकी हस्तांतरण के लिए मूल्य वृद्धि क्षेत्रिय मूल्यवृद्धि प्रणाली, खुला नवाचार दवाओं की खोज और अनपेक्षित लाभ सहित बाजार के लिए अनुवाद के सभी पहलुओं को शामिल करता है। यह इस बात को प्रदर्शित करता है। कि किस प्रकार कुछ उपकरण विकसित ढांचे (ए०सी०सी०ई0, ई0जी०ए०पी०पी०, टी01,टी04, पी०एच०जी० इन्टरप्राइज)से अलग कर दिये गए है और उसका कारण है संकीर्ण प्रयोजन तथा संभवतः उपकरणो का अतिव्यापन।

तृतीय अध्याय लर्निंग एडापटिंग लेवलिंग मॉडल का उपयोग करने पर पूरा विस्तृत प्रोटोकॉल विकसित करता है। इस प्रारंभिक अनुसंधान और विकास के बाद प्रौद्योगिकी हस्तांतरण से जुड़े व्यवसायिक मनुष्य के लिए मौका है कि वह ड्राइग बोर्ड तथा प्रतिपुष्टि फंदे को समझे जिसे पहले वह (एच०आइ0ए०)को (एच०टी०ए०)को (एच०एन०ए०) में (एच०टी०ए०) और (एच०आइ०ए०) व्यवसायिक मनुष्य से सम्पर्क करें। प्रक्रिया के दौरान सूचना एवं जन स्वास्थ्य जीनोमिक्स चक्र के मूल्य के संदर्भ फेम को ध्यान मे रखा जाता हैं यह मोटे तौर पर इसे परिभाषित सार्वजनिक निजी भागीदारी के लिए उपयोग किया जा सकता है। समय के साथ इसे अनुकूलित किया जा सकता है। इसके अलावा यह अध्याय समय सीमा के मुददों के साथ ही, संगतता समस्यओं के हल को भी प्रस्तुत करता है। चतुर्थ अध्याय भाग 'अ' में परिभाषित विशिष्ट उददेश्यों पर आधारित सामान्य निष्कर्ष देता है। 
भाग बः विकसित ढांचे की क्षमता का कार्यान्वयन परिदृश्यों को संबोधित करता है।

भाग-ब के अध्याय पांच ओमिक्स और जीव विज्ञान प्रणाली, व्यक्तिगत चिकित्सा और स्वास्थ्य को अपने रिश्ते की शुरुआत का एक परिणाम के रुप में जीव विज्ञान की जटिलता का वर्णन करता है। यह चिकित्सा (आइ०टी०ए०एम०) के एक मौजूदा यूरोपीय संघ के प्रमुख पायलट परियोजना (आइ0सी०टी०) भविष्य का एक उदाहरण का उपयोग कर विकसित ढांचे के कार्यान्वयन का प्रस्ताव करता है।

अध्याय छ: क्लैमाइडिया ट्कैमैटिस संक्रमण के संबंध मे आनुवांशिक मार्करो की कला की वर्तमान स्थिति का एक सिंहवलोकन देता है। सबफर्टिलिटी के वर्तमान नैदानिक प्रबंधन के लिए संक्रमण और प्रजवलन के आधार पर निदान अनुवांशिक मार्कर प्रोफाइल को जोड़ने की क्षमता अनुवादिक और नैदानिक मूल्य की जांच इसमें सम्मिलित है। यह अध्याय पारंपरिक क्लैमाइडिया और लर्निंग एडापटिंग लेवलिंग मॉडल मूल्य हेतु आनुवंशिक मार्करो का सार्वजनिक स्वास्थ्य में अनुवाद और इस क्षेत्र में अंतदृष्टि को दर्शाता है।

अध्याय सात गुणवत्ता आश्वासन प्रावधान और जीनोम आधारित सूचना और प्रौद्योगिकी (पी०एच०जी०इ०एन०) द्वितीय का उपयोग करने के लिए यूरोपीय सबसे अच्छे अभ्यास दिशा निर्देशो के विकास के लिए योगदान देता है और कुछ अवधारणाओं को भी साफ करता है। यहॉ पर शोध पत्र यूरोपीय नीतिगत दिशानिर्देशों में विकसित रुपरेखा के समावेश को दर्शाता है। अध्याय आठ मे परिभाषित विशिष्ट उददेश्यों के आधार पर भाग ब के सामान्य को निष्कर्ष देता है।

धारा 3 में विस्तार ढांचे के वैचारिक विकास की चर्चा है और पिछले अध्यायों के मुददों पर चर्चा है और यथास्थिति से परे कन्सेपटुलाइज और मॉडल की व्यापक पहुंच और स्वास्थ्य एकीकरण के लिए प्रौद्योगिकी हस्तांतरण से वैचारिक ढांचे के विकास के निष्कर्ष को निकाला है। यह अवधारणा सबूत के साथ वर्तमान में चल रही है, हालाकि धारा-4 पूरी शोध पत्र और ढांचे के जवाब जीनोम आधारित सूचना और प्रौद्योगिकी के वास्तविक समय प्रभावी और कुशल एकीकरण के बारे मे शोध के उददेश्यों के विकसित राज्यो में अंतिम निष्कर्ष बताता है। सहयोगियों के साथ अगले कुछ वर्षो मे परियोजना धारा-5 शोध पत्र के सारांश शोध लेखक के बायोडाटा के साथ प्रकाशन और आभार-पूर्ति की सूची देता है। 



\section{Curriculum Vitae}

Ir. Jonathan A. Lal (born 10 September 1982 in Allahabad, India) did his Bachelor of Technology degree (2001-2005) in Biotechnology with a specialization in Genetic Engineering from Sam Higginbottom Institute of Agriculture, Technology \& Sciences (formerly Allahabad Agricultural Institute AAIDU), India. During this time, he also worked as student trainees in the division of biological standardization at the Indian

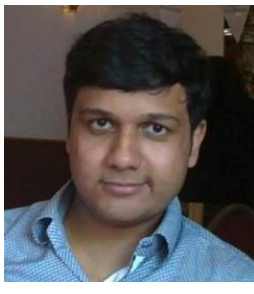
Veterinary Research Institute and as well as at the department of Genetic Medicine at Sir Ganga Ram Hospital, New Delhi. He also received training in 'Small Molecule Drug Design', 'Bioinformatics' and 'rDNA Technology' from the Institute of Bioinformatics and Applied Biotechnology, Bangalore, India.

He did his Master of Science in Life Science \& Technology (specialization Cell Diagnostics) from Delft University of Technology (TU Delft), The Netherlands from 2005 to 2007. This degree was in collaboration (joint degree) with Leiden University, The Netherlands. His master thesis was performed at the Institute of Biology, Leiden, in the Molecular \& Developmental Biology Group of which the research was on yeast genetics and protein-protein interactions studies. During his master's degree, he also did projects for Progentix BV, based in Bilthoven, and DNage BV, Leiden, The Netherlands.

After graduating from TU Delft, Ir. Lal worked as a Bioproduct Designer at the department of Biotechnology at TU Delft from 2007 to 2009 . Here he was involved in translating academic knowledge into industrial applications or bioproducts by applying integrative and creative approaches of Technological Product Design in the Life Sciences to design and evaluate products in the industrial context. During this time, he did a project for DSM and Paques BV and designed and worked on various patents for TU Delft. As a result he obtained his Engineering Doctorate or Professional Doctorate in Engineering degree in Bioproduct Design with a specialization in Pharma-Genomics \& Proteomics.

In 2009, Ir. Lal joined Maastricht University as the Project Manager of PHGEN II (Public Health Genomics European Network II), an EU DG SANCO funded project (no. 2008302) at the Institute for Public Health Genomics from October 2009 till 1 May 2013. PHGEN II was the follow-up of PHGEN I (DG SANCO) developing the first edition of "European Best Practice Guidelines for Quality Assurance, Provision and Use of Genome-based Information and Technologies". Ir. Lal managed, organized and assisted in 
developing and directing the evidence-based research from different European partners (over 22 partners) with regard to Public Health Genomics to formulate European guidelines for use of genomebased technologies in healthcare systems in Europe assisting EU Member States in policy making in the area of personalised healthcare and beyond. This also included financial as well as administrative and logistical aspects.

During his time at Maastricht University he worked also as a PhD researcher till 2013 in Public Health Genomics at the Institute for Public Health Genomics, GROW School for Oncology and Developmental Biology, Faculty of Health, Medicine \& Life Sciences at Maastricht University. His work was on 'Valorization in Public Health Genomics'. The thesis involves the pilot project grant from APOGEE CanGeneTest, Canada, regarding the efficient and effective integration of genome-based technologies into healthcare as well as PHGEN II. During this time he has co-written accepted FP7s among other grants. He was also involved in education activities at the university.

He was also assigned as the B-team member of the EU flagship pilot project ICT Future of Medicine (ITFoM) from 2011 to 2012 which had over 100 institutional members. Ir. Lal is a life time member for the 'Society for Biotechnologists', India, member of P3G (Population Project in Genomics) as well as a former member of KIVI NIRIA (The Royal Institute of Engineers) and member of the Dutch Network Research Pharmacogenetics both situated in The Netherlands. He is an alumni member of Delft University of Technology and LIFE (Delft) and involved in several international networks. He was also a guest reviewer for the "Special Issue on Synthetic Biology and Global Health" for the Systems and Synthetic Biology Journal 2012 and appeared in two interviews on Public Health Genomics. 


\section{Publications}

\section{List of Publications}

- Lal JA, Schulte in den Baümen T, Morré SA, Brand A. Public Health and Valorization of Genomebased Technologies: A new model. Journal of Translational Medicine 2011, 9:207.

- Brand A., Lal JA. European Best Practice Guidelines for Quality Assurance, Provision and Use of Genome-based Information and Technologies - the 2012 Declaration of Rome. Drug Metabolism and Drug Interactions 2012, 27(3):177-182.

- Soltani J, Lal JA, van Heusden GPH, Hooykaas PJJ. Nuclear localization of Agrobacterium VirD2 in the yeast Saccharomyces cerevisiae. Novin Genetics 2012, 29(2):157-164.

- Lal JA, Malogajski J, Verweij SP, de Boer P, Ambrosino E, Brand A, Oudburg S, Morré SA. Chlamydia trachomatis infections and subfertility: opportunities to translate host pathogen genomic data into public health. Public Health Genomics 2013, 16:50-61.

- Brand A, Lal J, Malats N, Gutierrez-Ibarluzea I. The Public Health Genomics European Network (PHGEN). The Newsletter of the British Society for Human Genetics 2013, 48, 53-55.

- Lal JA. Addressing the backlog of genome-based technologies in healthcare. The Newsletter of the British Society for Human Genetics 2013, 48, 60-61.

- Lal JA, Sudbrak R, Lehrach H, Brand A. Functional Dynamics: From Biological Complexity to Translation and Impact in Healthcare Systems. Journal of Computer Science and Systems Biology 2013, 6:88-92.

- Lal JA, Vaidya A, Gutiérrez-Ibarluzea I, Dauben HP, Angela Brand. The LAL Model: from theory to hypothesis of steps for implementation of basic genome-based evidences in personalized medicine. Personalized Medicine 2013, 10(7):683-701.

- Lal JA, Morré SA, Brand A. The overarching framework of translation and integration: a case for the LAL model. Personalized Medicine (In Press). 


\section{Conference Proceedings}

- Lal J. Valorization of genome-based technologies in public health. In proceedings of the 4th European Public Health Conference. European Journal of Public Health 2011, Vol. 21, Supplement 1. 10 November, Copenhagen.

- Vondeling H, Lal J, Douw Karla, Cassiman JJ, Brand A. Public Health Genomics and Health Technology Assessment. In proceeds of the 9th HTAi Annual Meeting. Gac Sanit. 2012, 26(Espec Congr 2):6.

- Lal JA. Valorization of Genomics in Public Health. Biomedica Life Science Summit 2011, Eindhoven Netherlands.

- Lal JA, Schulte in den Bäumen T, Morré SA, Brand A. Valorizing Genomics in Public Health. Genetica Retraite 2011, Kerkrade Netherlands. 


\section{Acknowledgements}

First of all I would like to thank my supervisors for their intellectual support and feasibility as well as flexibility in research progress. Without them, this work would have never been possible. I would specially like to thank Prof. Dr. Angela Brand for motivating me to merge my Delft expertise with Health Technology Assessment which became the primordial soup for the Learning-Adapting-Leveling model. Also her ideas merged with mine became killer instincts beyond just the development of the model. I would also like to appreciate the hard work of Prof. Dr. Servaas A. Morré with me, his problem-solving skills and industrial experience helped towards the boost I needed for my work. Angela and Servaas, I enjoyed all the late night 03:00 AM discussions and whole night marathons of research. We were a unique and one of kind team. Thank you both for the opportunities which came down the road. I would also like to thank my co-authors for their useful feedback and collaboration. Also, I would like to thank PHGEN II and APOGEE Canada for their investments in me. I want to extend my thanks to Prof. Dr. Joep Geraedts for his constant support to our Institute which got embedded in his department, through which my research was made possible.

I would like to thank my parents for their continued support and guidance. They have been always been there for me in thick and thin. Thank you. When in a problem they were always the loving resolvers. Without their support again, I would have not been here in the first place. Love you Mom and Dad! You both have done so much for me that I will never be able to grasp the love you have showered upon me. Guess that will always be with children - your striving to be obedient son.

I am thankful to my beautiful wife Abhilasha. Her sacrifice, service, love and including hard work with the kids at home while I was in mental lockdown towards my research and associated projects like PHGEN II, ITFOM, APOGEE, grants, international conferences, etc. has contributed to the product of the thesis. Those long hours spent at work, overnight and outside of Maastricht were made only possible through her patience and love towards me. Thank you darling, I really appreciate it and love you for eternity!

This thesis is dedicated to my children Joyce and Joel born in Delft and Maastricht respectively. Their love and patience ("when's Papa coming home?", "please don't go Papa", "love you Papa", etc.) will forever be engrained in me.

I would like to thank Annelies Plugge for the translation of the Dutch summary and Samuel Richmond Saxena and Shailesh Marker for the translation of the Hindi summary. Appreciate it.

Lastly, I would like to thank my Church and my God to whom I am eternally grateful and in eternal debt. The best guidance once could ever get. THANK YOU and LOVE YOU!

"Happy is the man who finds wisdom, and the man who gains understanding" 


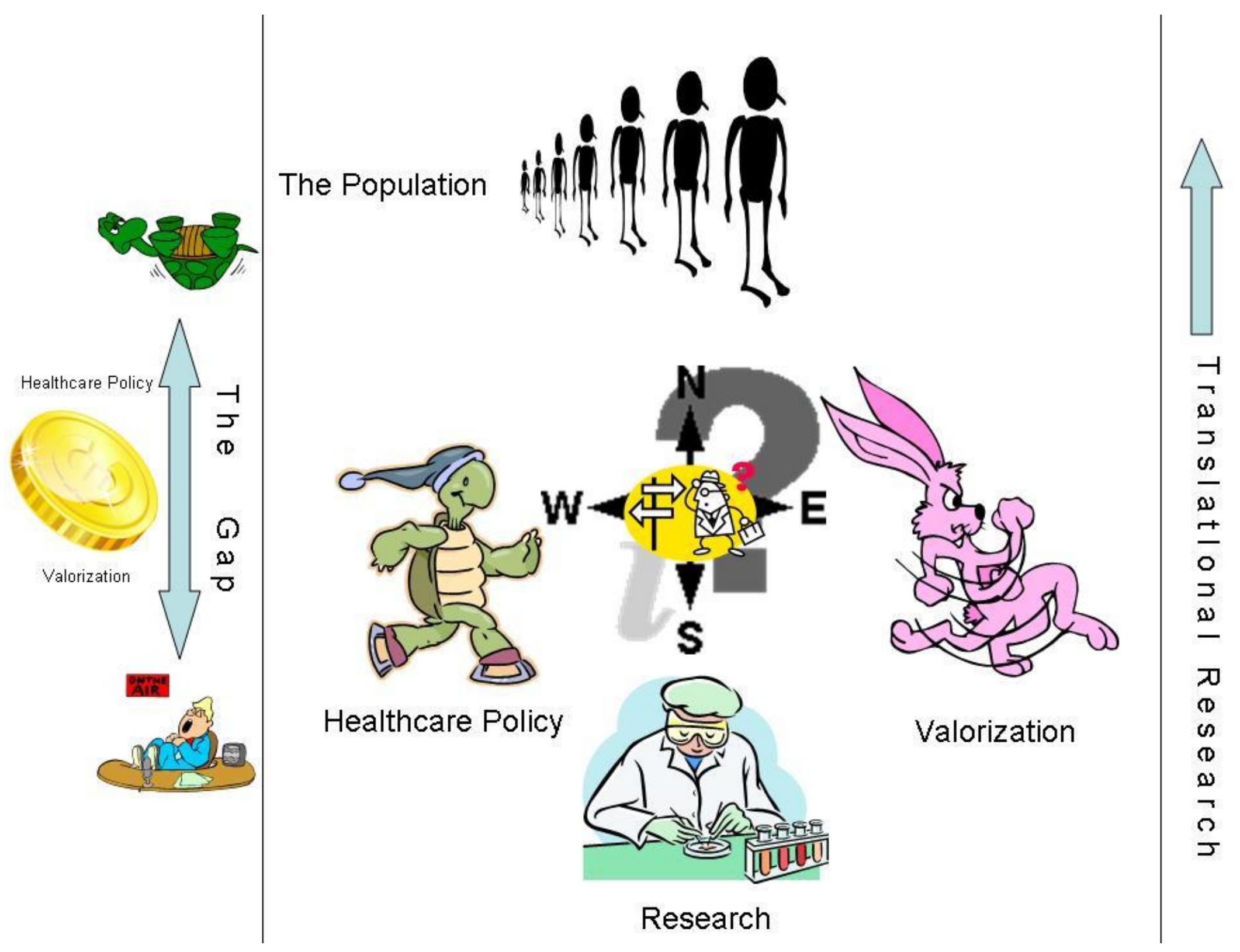

